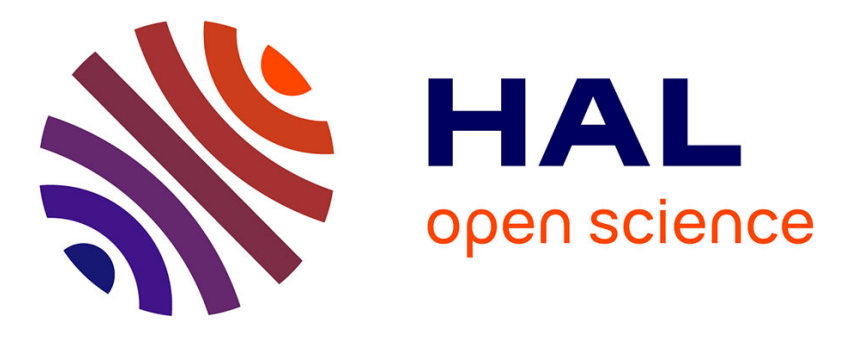

\title{
Le gisement paléolithique moyen et les séquences pléistocènes de Villiers-Adam (Val-d'Oise) : chronostratigraphie, environnement et implantations humaines
}

Jean-luc Locht, Pierre Antoine, Jean-Jacques Bahain, Gabriel Dwrila, Pascal Raymond, Nicole Limondin-Lozouet, Agnès Gauthier, Nick Debenham, Manfred Frechen, Denis-Didier Rousseau, et al.

\section{- To cite this version:}

Jean-luc Locht, Pierre Antoine, Jean-Jacques Bahain, Gabriel Dwrila, Pascal Raymond, et al.. Le gisement paléolithique moyen et les séquences pléistocènes de Villiers-Adam (Val-d'Oise) : chronostratigraphie, environnement et implantations humaines. Gallia Préhistoire - Archéologie de la France préhistorique, 2003, 45, pp.1-111. 10.3406/galip.2003.2036 . hal-02353480

\author{
HAL Id: hal-02353480 \\ https://hal.science/hal-02353480
}

Submitted on 10 Feb 2021

HAL is a multi-disciplinary open access archive for the deposit and dissemination of scientific research documents, whether they are published or not. The documents may come from teaching and research institutions in France or abroad, or from public or private research centers.
L'archive ouverte pluridisciplinaire HAL, est destinée au dépôt et à la diffusion de documents scientifiques de niveau recherche, publiés ou non, émanant des établissements d'enseignement et de recherche français ou étrangers, des laboratoires publics ou privés.

\section{(ㅇ)(1) $\$$}

Distributed under a Creative Commons Attribution - NonCommercial - NoDerivatives 44.0 


\section{Le gisement paléolithique moyen et les séquencess pléistocènes de} Villiers-Adam (Val-d 'Oise) : chronostratigraphie, environnement et implantations humaines

Jean-Luc Locht, Pierre Antoine, Jean-jacques Bahain, Gabriel Dwrila, P. Raymond, Nicole Limondin-Lozouet, Agnès Gauthier, Nick Debenham, Manfred Frechen, Denis Didier Rousseau, Christine Hatté, Paul Haesaerts, Hans Metsdagh

\section{Citer ce document / Cite this document :}

Locht Jean-Luc, Antoine Pierre, Bahain Jean-jacques, Dwrila Gabriel, Raymond P., Limondin-Lozouet Nicole, Gauthier Agnès, Debenham Nick, Frechen Manfred, Rousseau Denis Didier, Hatté Christine, Haesaerts Paul, Metsdagh Hans. Le gisement paléolithique moyen et les séquencess pléistocènes de Villiers-Adam (Val-d 'Oise) : chronostratigraphie, environnement et implantations humaines. In: Gallia préhistoire, tome 45, 2003. pp. 1-111;

doi : https://doi.org/10.3406/galip.2003.2036

https://www.persee.fr/doc/galip_0016-4127_2003_num_45_1_2036

Fichier pdf généré le 28/03/2019 


\title{
Zusammenfassung
}

Die 1996 beim Autobahnbau entdeckte mittelpaläolithische Fundstelle von Villiers-Adam mit ihrer pleistozänen Schichtenabfolge bot erstmals im Département Val-d'Oise die Gelegenheit fur eine multidisziplinäre Untersuchung. Der in die frühe Weichsel-Kaltzeit (105 $000 \mathrm{BP}$ cal.) datierte Hauptbelegungshorizont des Fundplatzes Petit Saule wurde auf einer Fläche von $3866 \mathrm{~m}^{2}$ ausgegraben. Die Steingeräteindustrie des Platzes ist durch unterschiedliche Produktionstechniken hier hergestellter Levalloisspitzen gekennzeichnet. Durch die Untersuchung der Fundverteilung der Steinartefakte gelingt der Nachweis räumlicher Organisation für eine Fündstelle vom Anfang der letzten Vergletscherung. Außerdem liefern die Stratigraphien bedeutende Umweltdaten fur einen etwa 200000 jahre umfassenden Zeitabschnitt, der die letzten beiden zyklischen Abfolgen von Interglazial und Glazial abdeckt. Durch die erarbeiteten Querverbindungen zu anderen nordwesteuropäischen Profilen wird Villiers-Adam zu einem wichtigen Bezugspunkt fur die Kenntnis des Quarters in Nordfrankreich.

\begin{abstract}
Discovered in 1996 during motorway construction works, the Middle Palaeolithic site of Villiers-Adam and associated Pleistocene sequences provided an opportunity to a multidisciplinary study for the first time in the Val-d'Oise department. The main Early Wechselian archaeological level (105000 ka BP) of the Petit Saule site has been excavated over an area of $3866 \mathrm{~m}^{2}$. It's characterized by a Levallois points production, via various methods. The study of the distribution of the lithic artefacts has yielded important informations about the internal structuration of the site. The stratigraphic sequence gave important palaeoenvironmental data about the last 200000 years which correspond to a period covering two interglacial-glacial cycles (IS 7 to 2). Due to correlations between this site and other North-Western European sequences, Villiers-Adam is a reference for the knowledge of the Northern France Quaternary.
\end{abstract}

\section{Résumé}

Découvert en 1996 lors de travaux autoroutiers, le gisement paléolithique moyen de Villiers-Adam et les coupes pléistocènes qui y sont associées ont offert l'opportunité, pour la première fois dans le Vald'Oise, d'une étude pluridisciplinaire. Le niveau d'occupation principal du site du Petit Saule, daté du Weichsélien ancien (105 $000 \mathrm{ka} \mathrm{BP}$ ), a été fouillé sur une surface de $3866 \mathrm{~m}^{2}$. Son industrie lithique est caractérisée par la production de pointes Levallois via des schémas opératoires variés. L'étude de la répartition des vestiges lithiques a permis de mettre en évidence une structuration de l'espace sur un gisement du début de la dernière glaciation. Par ailleurs, les séquences stratigraphiques ont livré d 'importantes données paléoenvironnementales sur une période de temps d'environ 200000 ans couvrant les deux derniers cycles interglaciaire-glaciaire (SI 7 à 2). Les corrélations réalisées avec d'autres profils du Nord-Ouest européen font de Villiers-Adam une référence pour la connaissance du Quaternaire dans le nord de la France. 


\section{LE GISEMENT PALÉOLITHIQUE MOYEN ET LES SÉQUENCES PLÉISTOCÈNES DE VILLIERS-AdAM (VAL-D’OISE)}

\section{Chronostratigraphie, environnement et implantations humaines}

Jean-Luc Locht, Pierre ANToIne, Jean-Jacques Bahain, Gabriel Dwrila, Pascal RAYMOND, Nicole Limondin-Lozouet, Agnès GAUTHIER, Nick Debenham, Manfred Frechen, Denis-Didier Rousseau, Christine Hatté, Paul HaEsaerTs et Hans Metsdagh

Présentation (p. 3-8)

Jean-Luc Locht, Pierre ANTOINE et Jean-Jacques BaHAIN

Stratigraphie et sédimentologie (p. 9-20)

Pierre Antoine, Jean-Jacques Bahain, Paul Haesaerts et Hans Metsdagh

Données bioclimatiques et géochimiques (p. 20-49)

Analyse pollinique, Agnès GAUTHIER

Étude des malacofaunes du Petit Saule et du Chamesson, Nicole Limondin-Lozouet

Comparaison des données palynologiques et malacologiques, Agnès GaUtHIER et Nicole Limondin-LozoueT Isotopie organique, Christine HaTtÉ

Évolution pédosédimentaire, interprétation chronostratigraphique et paléoenvironnementale (p. 49-62)

Pierre Antoine, Jean-Jacques Bahain, Nicole Limondin-Lozouet, Agnès Gauthier, Nick Debenham, Manfred Frechen, Denis-Didier Rousseau, Christine Hatté, Paul Haesaerts et Hans Metsdagh

Chronostratigraphie et environnement du site paléolithique de Villiers-Adam, le Petit Saule (p. 62-66)

Pierre Antorne, Jean-Jacques Bahain, Nicole Limondin-Lozouet, Agnès Gauthier, Nick Debenham, Manfred Frechen, Denis-Didier Rousseau, Christine Hatté, Paul Haesaerts, Hans Metsdagh et Jean-Luc LocHT

Études archéologiques (p. 67-104)

Jean-Luc Locht, Gabriel Dwrila et Pascal Raymond

Pierre ANTOINE, UMR 8591 du CNRS, Laboratoire de géographie physique, 1 place Aristide-Briand, F-92195 Meudon Cedex. Mél : Pierre.Antoine@cnrs-bellevue.fr.

Jean-Jacques BAHAIN, Institut de paléontologie humaine, Laboratoire de préhistoire du Muséum national d'histoire naturelle, UMR 6569 du CNRS, 1 rue René-Panhard, F-75013 Paris. Mél : bahain@cimrs1.mnhn.fr.

Nick Debenham, TL Quaternary Survey, 19 Leonard-Avenue, Nottingham NG5 2LW, UK.

Mél : n.debenham@qtls.globalnet.co.uk.

Gabriel DwRILA, INRAP, 32 rue Delizy, F-93500 Pantin. 
Manfred FRECHEN, Institut für Geowissenschaftliche Gemeinschaftsaufgaben (GGA), S3 Geochronologie und Isotopenhydrologie, Stilleweg 2, 30631 Hannovre, Allemagne. Mél : M.Frechen@gga-hannover.de

Agnès GAUTHIER, UMR 8591 du CNRS, Laboratoire de géographie physique, 1 place Aristide-Briand, F-92195 Meudon Cedex. Mél : agnes.gauthier@cnrs-bellevue.fr.

Paul HAESAERTS, Institut royal des sciences naturelles, 29 rue Vautier, Bruxelles, Belgique.

Christine HATTÉ, Laboratoire des sciences du climat et de l'environnement, UMR CEA-CNRS 1572, Avenue de la Terrasse, F 91198 Gif-sur-Yvette Cedex. Mél : Christine.Hatte@lsce.cnrs-gif.fr.

Nicole Limondin-Lozouet, UMR 8591 du CNRS, Laboratoire de géographie physique, 1 place Aristide-Briand, F-92195 Meudon Cedex. Mél : limondin@cnrs-bellevue.fr.

Jean-Luc LOCHT, INRAP, 518 rue Saint-Fuscien, F-80000 Amiens et ESA 8018 du CNRS, Préhistoire et Quaternaire, UFR de géographie, Université des sciences et technologies de Lille, Avenue Paul-Langevin, F-59655 Villeneuve-d'Ascq Cedex. Mél : nord-picardie@inrap.fr.

Hans Metsdagh, Institut royal des sciences naturelles, 29 rue Vautier, Bruxelles, Belgique.

Pascal RAYMOND, INRAP, 32 rue Delizy, F-93500 Pantin.

Denis-Didier RousSEaU, Université Montpellier-II, UMR 5554 du CNRS, Paléoenvironnements et palynologie, Institut des sciences de l'Évolution, case 61, place Bataillon, F-34095 Montpellier Cedex 05.

Mél : denis@dstu.univ-montp2.fr.

Mots-clés. Paléolithique moyen, pointe Levallois, chronostratigraphie, Weichsélien ancien, approche pluridisciplinaire.

Résumé. Découvert en 1996 lors de travaux autoroutiers, le gisement paléolithique moyen de Villiers-Adam et les coupes pléistocènes qui y sont associées ont offert l'opportunité, pour la première fois dans le Val-d'Oise, d'une étude pluridisciplinaire. Le niveau d'occupation principal du site du Petit Saule, daté du Weichsélien ancien (105000 ka BP), a été fouillé sur une surface de $3866 \mathrm{~m}^{2}$. Son industrie lithique est caractérisée par la production de pointes Levallois via des schémas opératoires variés. L'étude de la répartition des vestiges lithiques a permis de mettre en évidence une structuration de l'espace sur un gisement du début de la dernière glaciation. Par ailleurs, les séquences stratigraphiques ont livré d'importantes données paléoenvironnementales sur une période de temps d'environ 200000 ans couvrant les deux derniers cycles interglaciaire-glaciaire (SI 7 à 2). Les corrélations réalisées avec d'autres profils du NordOuest européen font de Villiers-Adam une référence pour la connaissance du Quaternaire dans le nord de la France.

Key-words. Middle Palaeolithic, Levallois point, chronostratigraphy, Early Weichselian, multidisciplinar approach.

\begin{abstract}
Discovered in 1996 during motorway construction works, the Middle Palaeolithic site of Villiers-Adam and associated Pleistocene sequences provided an opportunity to a multidisciplinary study for the first time in the Val-d'Oise department. The main Early Wechselian archaeological level (105 $000 \mathrm{ka} \mathrm{BP})$ of the Petit Saule site has been excavated over an area of $3866 \mathrm{~m}^{2}$. It's characterized by a Levallois points production, via various methods. The study of the distribution of the lithic artefacts has yielded important informations about the internal structuration of the site. The stratigraphic sequence gave important palaeoenvironmental data about the last 200000 years which correspond to a period covering two interglacial-glacial cycles (IS 7 to 2). Due to correlations between this site and other NorthWestern European sequences, Villiers-Adam is a reference for the knowledge of the Northern France Quaternary.
\end{abstract}

Schlagwörter. Mittelpaläolithikum, Levalloisspitzen, Chronostratigraphie, frühe Weichsel-Kaltzeit, multidisziplinärer Ansatz.

Zusammenfassung. Die 1996 beim Autobahnbau entdeckte mittelpaläolithische Fundstelle von Villiers-Adam mit ihrer pleistozänen Schichtenabfolge bot erstmals im Département Val-d'Oise die Gelegenheit für eine multidisziplinäre Untersuchung. Der in die frühe Weichsel-Kaltzeit (105000 BP cal.) datierte Hauptbelegungshorizont des Fundplatzes Petit Saule wurde auf einer Fläche von $3866 \mathrm{~m}^{2}$ ausgegraben. Die Steingeräteindustrie des Platzes ist durch unterschiedliche Produktionstechniken hier hergestellter Levalloisspitzen gekennzeichnet. Durch die Untersuchung der Fundverteilung der Steinartefakte gelingt der Nachweis räumlicher Organisation für eine Fundstelle vom Anfang der letzten Vergletscherung. Außerdem liefern die Stratigraphien bedeutende Umweltdaten für einen etwa 200000 Jahre umfassenden Zeitabschnitt, der die letzten beiden zyklischen Abfolgen von Interglazial und Glazial abdeckt. Durch die erarbeiteten Querverbindungen zu anderen nordwesteuropäischen Profilen wird Villiers-Adam zu einem wichtigen Bezugspunkt für die Kenntnis des Quartärs in Nordfrankreich. 


\section{PRÉSENTATION}

\section{LOCALISATION GÉOGRAPHIQUE ET HISTORIQUE DES TRAVAUX}

Les séquences pléistocènes et le gisement paléolithique moyen de Villiers-Adam se situent à environ $35 \mathrm{~km}$ au nord de Paris, à l'extrémité occidentale de la Plaine de France (fig. 1 et 2), à une dizaine de kilomètres au nord-est de Pontoise (Val-d'Oise). Le site paléolithique et les profils étudiés se répartissent sur le versant exposé au nord - nord-est (versant en pente douce) d'une vallée sèche asymétrique affluente de la rive gauche de la vallée de l'Oise, située 3 à $4 \mathrm{~km}$ à l'ouest nord-ouest de la zone de travail (fig. 3).

Les études stratigraphiques et environnementales présentées dans cet article ont été menées en parallèle avec les interventions archéologiques et les travaux de terrassement effectués dans le cadre de l'opération Francilienne nord - liaison Cergy-Roissy (LCR) entre 1995 et $2000^{\prime}$. Ces travaux ont permis en 1995 la découverte par V. Krier (AFAN) au lieu-dit Le Chamesson à Villiers-Adam d'une séquence lœssique présentant des paléosols du Début glaciaire weichsélien, ce qui a motivé le développement de recherches plus poussées dans cette zone, préalablement au démarrage des travaux de terrassement. Ces investigations ont permis la découverte en avril 1996 du premier gisement paléolithique en stratigraphie du département du Val-d'Oise, toujours à Villiers-Adam au lieu-dit Le Petit Saule (fig. 3) (Bahain et al., 1996). Ce site fit l'objet d'une fouille de sauvetage d'août à novembre 1996 (Locht et al., 1997) ${ }^{2}$. La poursuite des

1. I a coordination scientifique de cette opération était assuréc conjointement par François (ientili (AFAN Centre - Île-de-France), Franck Suméra (service départemental de l'Archéologie du Val-d'Oise) et Jean-Marc Gouédo (service régional de l'Archéologie d'Île-de-France) que les auteurs tiennent à remercier.

2. Cette fouille a cté réalisée sous la direction de Jean-Luc Iocht (AFAN). Y ont participé Jean-Jacques Bahain, Gabricl Dwrila, Fabrice Marti, Paul Nestéroff, Pascal Raymond (tous AFAN) et Jean-Pierre labbé (bénévole).

Fig. 1 - Localisation du gisement paléolithique moyen et des séquences pléistocènes de Villiers-Adam: 1, dans le nord de la France; 2 , en Île-de-France; 3, dans le Val-d'Oise; 4, sur le tracé de la liaison Cergy-Roissy' (A 104).
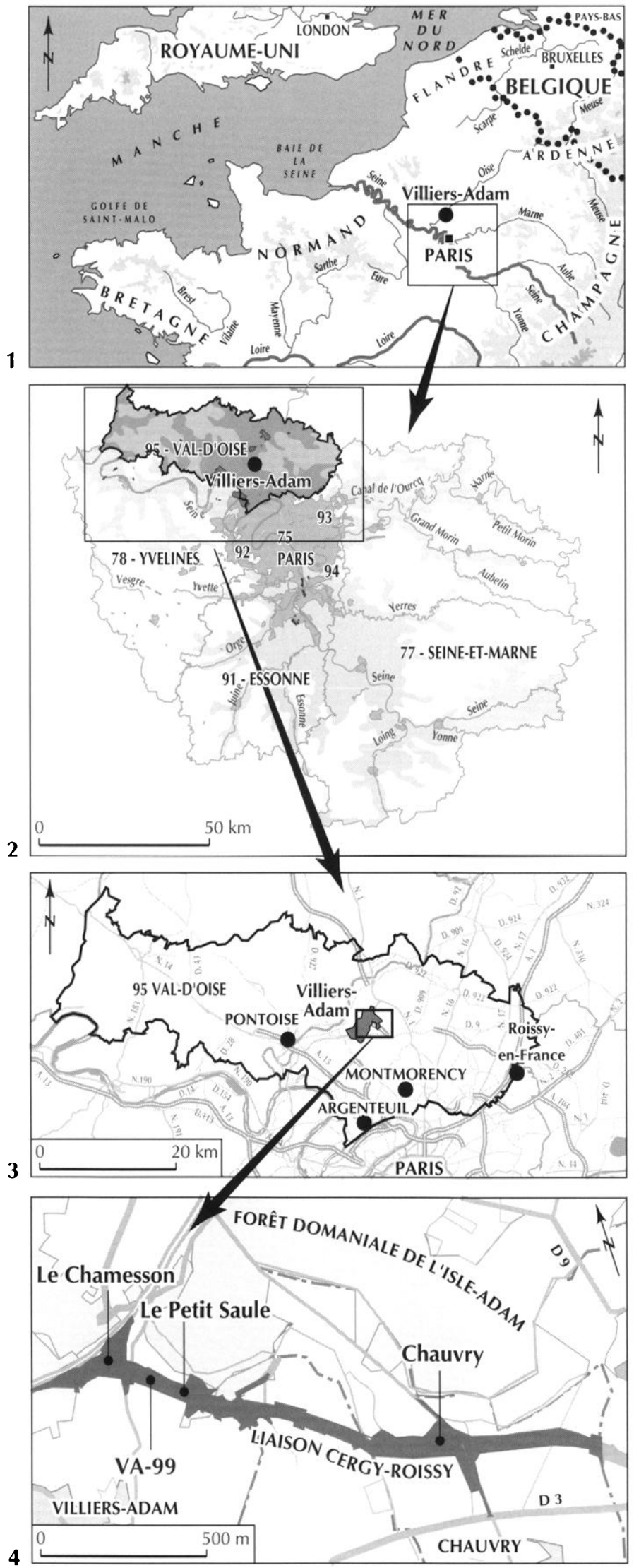


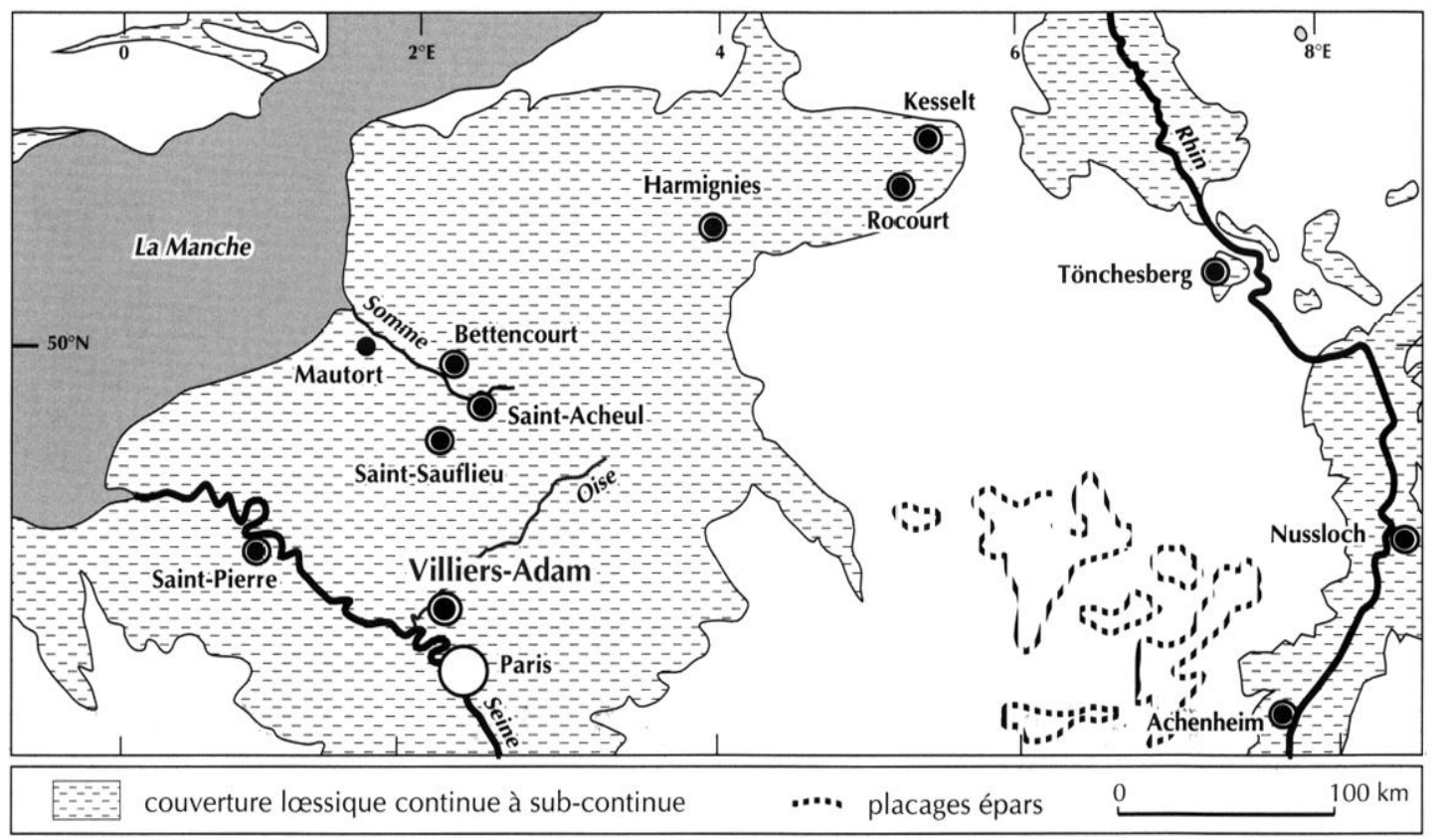

Fig. 2 - Localisation du secteur de Villiers-Adam dans la France septentrionale et cartographie simplifiée de l'extension géographique des loess du Pléniglaciaire supérieur weichsélien (d'après Antoine et al., 1999, modifié).

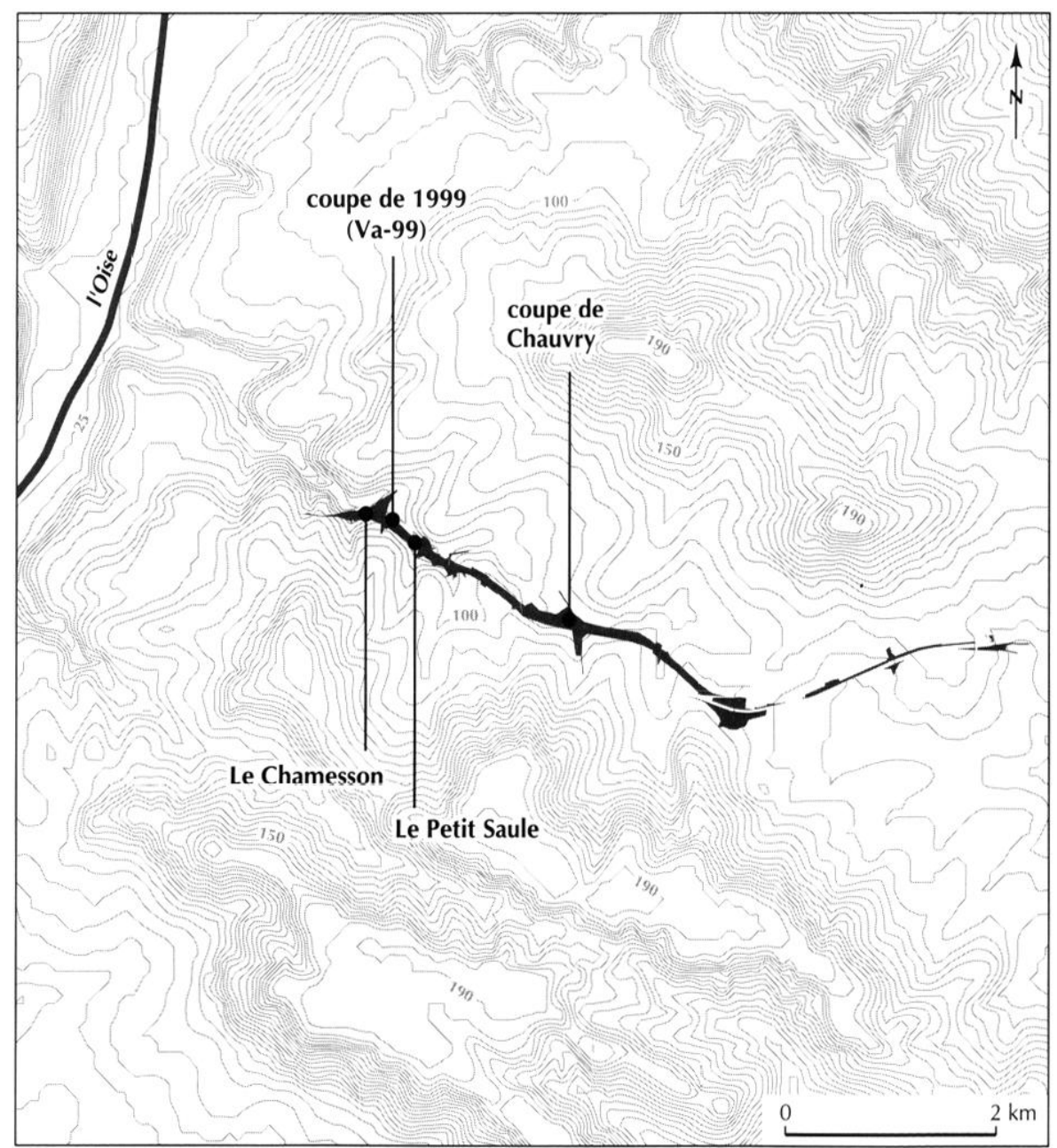

Fig. 3 - Localisation et contexte morphologique des sites de Villiers-Adam (Le Chamesson, VA-99 et Le Petit Saule) et de Chaurry. 
terrassements a ensuite permis d'observer de nouveaux profils stratigraphiques tout d'abord en 1997 sur la commune de Chauvry, environ $1 \mathrm{~km}$ à l'est du Chamesson, puis à nouveau à Villiers-Adam entre Le Chamesson et Le Petit Saule en 1999.

Outre un intérêt archéologique certain, puisque relativement peu de sites du Paléolithique moyen d'Île-deFrance ont fait l'objet de fouilles au cours des dernières décennies, cette étude constitue une contribution originale à la connaissance du Quaternaire d'une région où les données disponibles sur le Pléistocène sont essentiellement liées à l'établissement de la carte géologique dans les années 1970 (Soyer, 1959; Cavelier, 1967 ; Pomerol, Feugueur, 1974 ; Wins, Monciardini, 1979), puis plus récemment à une synthèse réalisée par P. Lebret et V. Halbout (1991).

\section{CONTEXTE ARCHÉOLOGIQUE}

Le nombre de sites du Paléolithique moyen découverts en Île-de-France ces dernières années reste faible au vu du grand nombre de sauvetages archéologiques qui y ont été effectués. Seuls deux autres gisements ont été fouillés récemment : Arvigny, en Seine-et-Marne, dans un contexte limoneux de plateau (Gouédo et al., 1994) et Maisons-Alfort, dans le Val-de-Marne, dans un contexte fluviatile (Durbet et al., 1997).

Pourtant, les premières découvertes d'industries paléolithiques en Val-d'Oise remontent au XIX ${ }^{\mathrm{e}}$ siècle. Elles concernaient alors principalement des sites associés aux terrasses fluviatiles de l'Oise. La collection la plus importante provient de plusieurs petites carrières exploitées autrefois au lieu-dit Le Bois de la Ballastière à Cergy. Ce gisement, situé sur la basse terrasse de l'Oise, fut découvert par $\mathrm{A}$. Laville, qui y a récolté un outillage lithique datable du Paléolithique inférieur et moyen associé à de la faune (fig. 4) (Laville, 1898, 1902 ; Rutot, 1902). Le site fut réétudié à la fin des années 1960 par $R$. Chrétien qui y récolta une industrie moustérienne. Dans son étude sur les formations alluviales du bassin de Paris, J.-P. Michel (1972) attribua à cette industrie un âge Würm I.

Bien que les découvertes des trente dernières années soient rares dans ce département (Bonis et al., 1992), la carte archéologique du Val-d'Oise recense environ une quarantaine de sites du Paléolithique moyen (P. Soulier et F. Suméra, comm. pers.). Ils correspondent en général à une ou plusieurs pièces lithiques trouvées fortuitement lors de prospections pédestres ou de fouilles archéologiques de sites d'âge plus récent. Ces découvertes de surface sont dépourvues de tout contexte chronostratigraphique, ce qui limite les possibilités d'une étude fiable. L'attribution culturelle est alors proposée à partir de la présence du débitage et des nucléus Levallois, et sur la reconnaissance de certains bifaces (petits bifaces cordiformes attribué au Moustérien de tradition acheuléenne, bifaces lancéolés du Micoquien).

À l'est du département, le Pays de France, prospecté depuis plusieurs années par une association départementale, la Jeunesse préhistorique et géologique de France (JPGF), a récemment fait l'objet d'une étude particulière (Cecchini, 1990). L'auteur y recense des gisements du Paléolithique inférieur et moyen situés dans le bassin hydrologique du Crould, petit cours d'eau qui draine le Val-d'Oise entre Goussainville et SaintDenis, où il se jette dans la Seine, et en dresse un premier bilan typologique. Il s'agit encore une fois exclusivement de ramassages de surface et la distinction entre les industries est faite sur la base de la patine. Le rattachement de certaines industries à la famille moustérienne est également basé sur la présence du débitage Levallois et de certaines catégories d'outils retouchés (racloirs, pointes moustériennes).

Sur la commune de Chauvry, voisine de celle de Villiers-Adam, un abondant matériel paléolithique moyen est récolté en surface par C. Mariette depuis 1982, à environ un kilomètre à vol d'oiseau de la fouille du Petit Saule (Charpentier, 1982). La matière première utilisée par les Paléolithiques est le silex, tertiaire ou secondaire. Les nucléus unipolaires sont fréquents, les pointes Levallois nombreuses, ainsi que des lames à dos cortical ou de débitage. L'outillage est dominé par les racloirs, surtout simples, mais également doubles convergents ou déjetés.

Par ailleurs, un site du Paléolithique moyen a déjà été découvert sur le territoire même de la commune de Villiers-Adam par C. Toupet en 1982 (Toupet in Bonis et al., 1992). Il se situe au lieu-dit Le Buisson du Val, à environ $1,5 \mathrm{~km}$ à l'ouest du Petit Saule. Là encore, l'occupation paléolithique est connue uniquement par des ramassages de surface, car les sondages ouverts à l'endroit de la découverte se sont révélés négatifs. La série lithique, peu abondante, est taillée exclusivement 


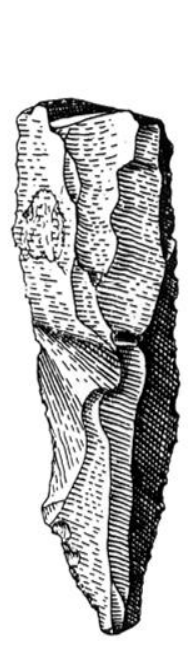

1

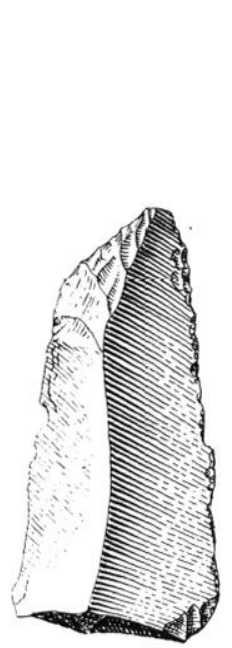

2

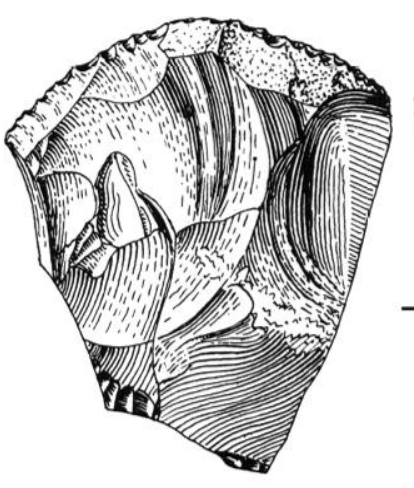

6

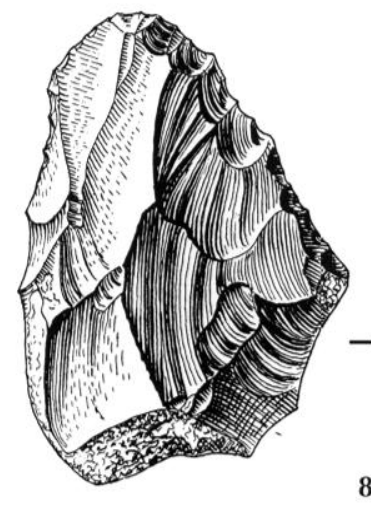

8
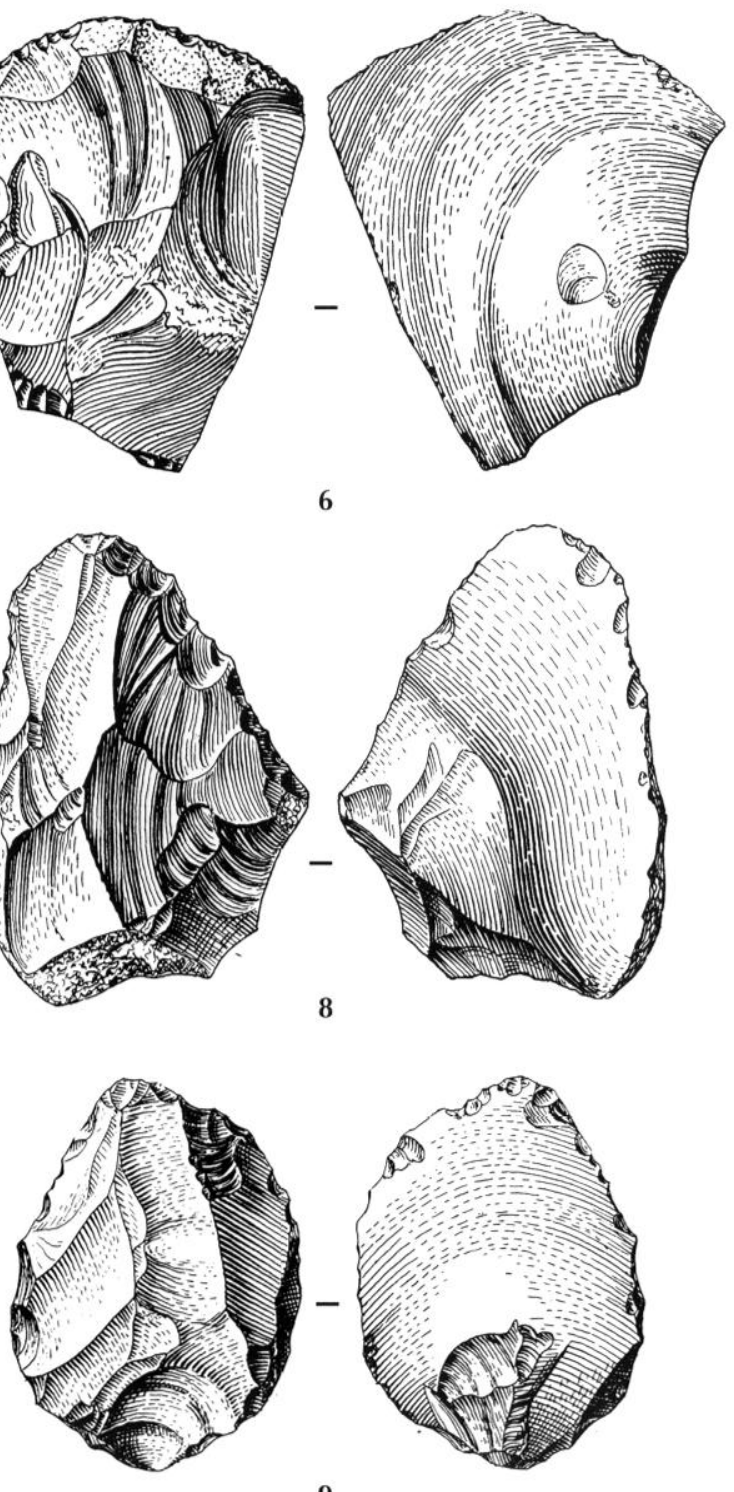

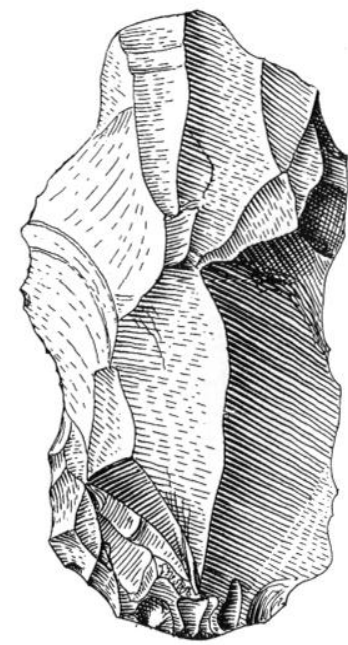

3

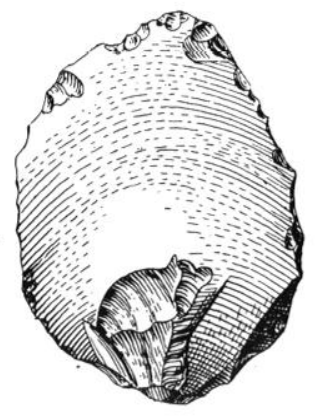

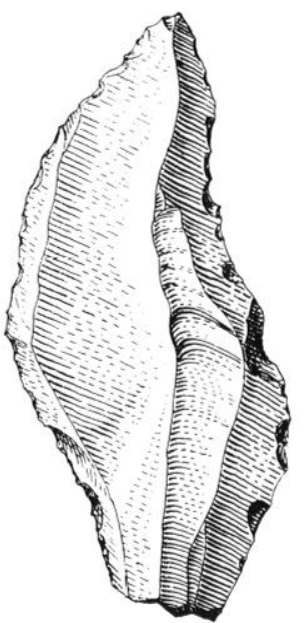

4
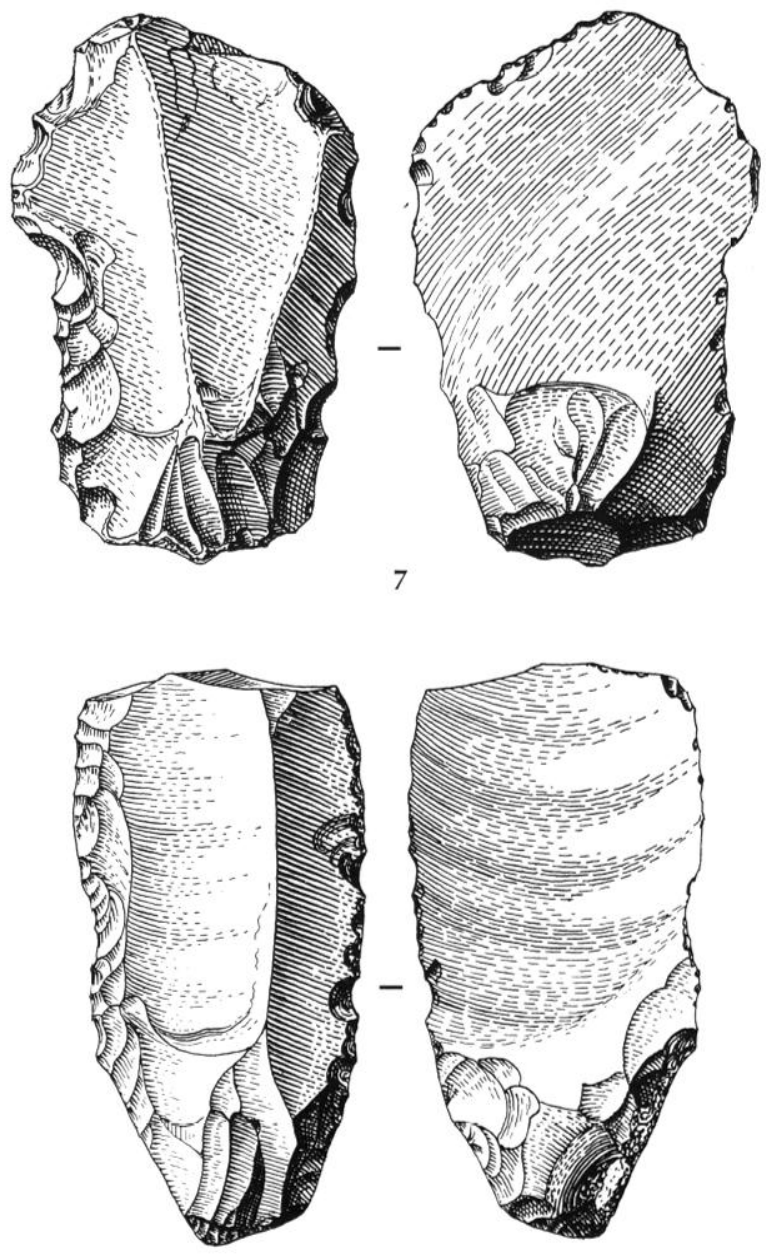

10

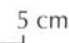

Fig. 4 - Cergy, Le Bois de la Ballastière. Artefacts découverts à la fin du XIX siècle par A. Laville (d'après Rutot, 1902, mise en page modifiée) : 1, 2, lames ; 3, éclat Levallois ; 4, lame ; 5, nucléus ; 6, grattoir à tranchant transversal ; 7, lame épaisse ; 8, éclat ; 9, racloir ; 10, racloir sur lame. 

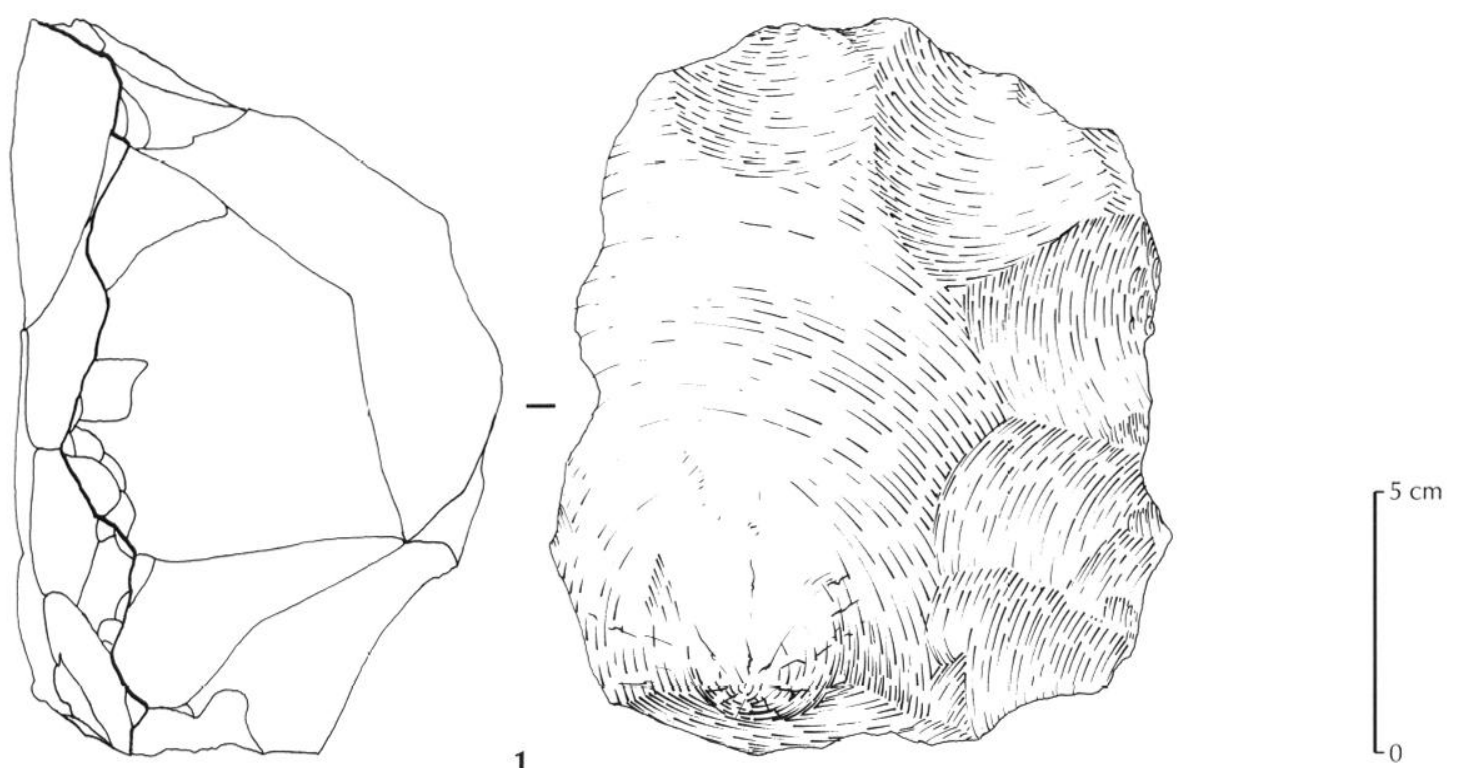

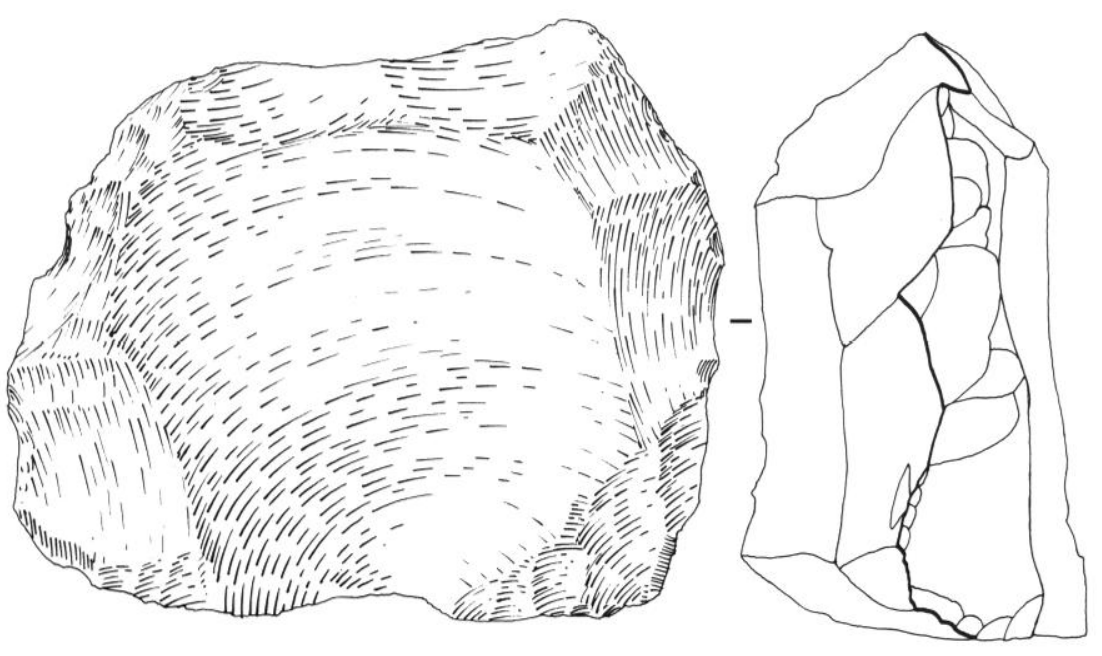

2

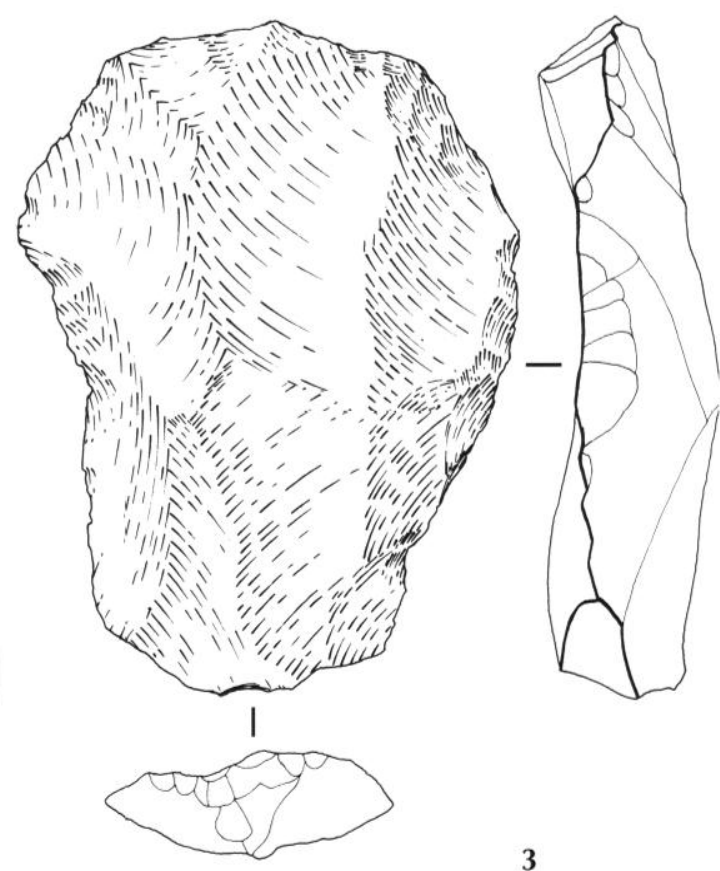

Fig. 5 - Villiers-Adam, Le Buisson du Val. Artefacts en grès découvert en 1982 par C. Toupet : 1, gros éclat réutilisé comme nucléus avec traces de préparation de convexités latérales et distale; 2, nucléus Levallois à éclat préférentiel ; 3, éclat Levallois préférentiel.

dans le grès stampien, matériau d'origine locale de bonne qualité, et se caractérise par la présence de nucléus Levallois à éclat préférentiel de très grande taille, ainsi que par celle d'éclats Levallois (fig. 5). Aucun contexte chronostratigraphique ne peut être associé à cette industrie, ce qui rend les comparaisons difficiles.
Pour l'une des premières fois en Île-de-France dans un contexte de versant, le gisement du Petit Saule et les importantes séquences pléistocènes du Chamesson, de Chauvry et de Villiers-Adam 99 (VA-99) ont ainsi offert l'opportunité d'étudier un gisement du Paléolithique moyen dans un contexte chronostratigraphique qui pourra servir de référence régionale. 


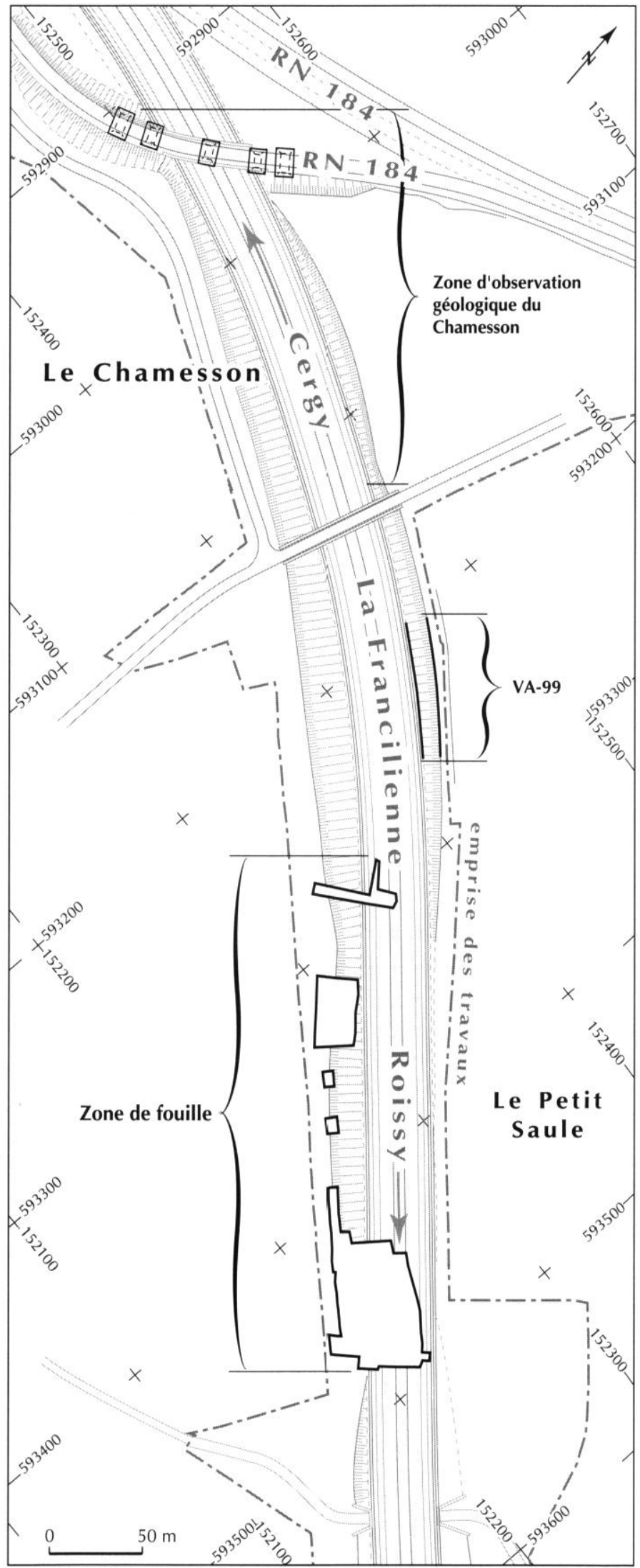

Fig. 6 - Localisation des profils étudiés (Le Chamesson, VA-99 et Le Petit Saule) sur le tracé de la LCR (A 104).
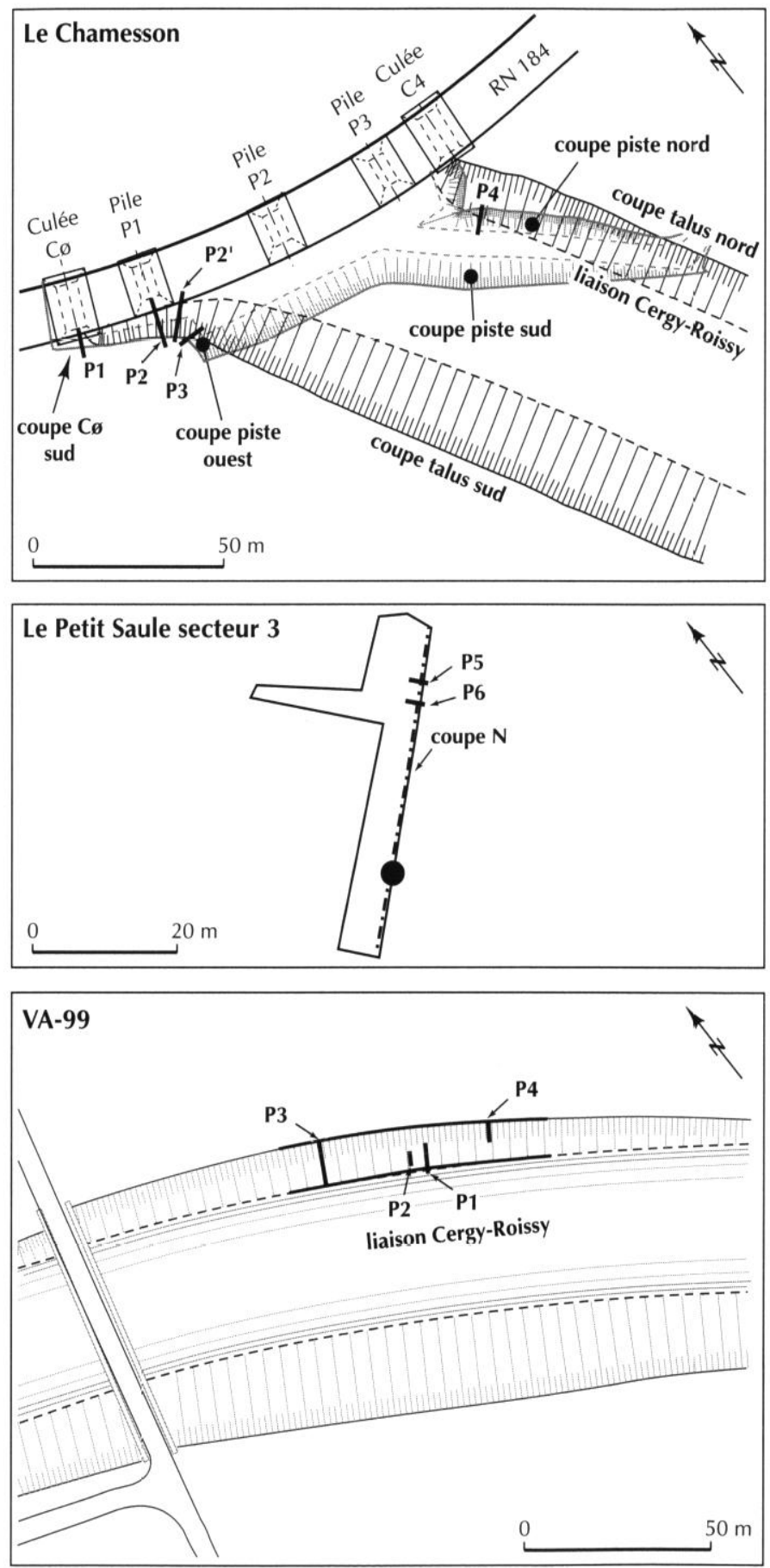

Fig. 7 - Plans des sites de Villiers-Adam : Le Chamesson, VA-99 et Le Petit Saule avec localisation des profils étudiés pour l'élaboration de la stratigraphie. 


\section{STRATIGRAPHIE ET SÉDIMENTOLOGIE}

\section{INTRODUCTION}

L'étude de la stratigraphie de la séquence pléistocène de Villiers-Adam s'est tout d'abord concentrée sur les profils du Chamesson (profils P1-P4), puis sur les coupes du gisement paléolithique du Petit Saule (profils P5-P6) (fig. 3, 6 et 7).

Ces premières investigations menées en 1996 ont rapidement permis de souligner l'intérêt exceptionnel de la séquence de Villiers-Adam pour l'étude du Pléistocène du Bassin parisien, et ont débouché sur une première interprétation chronostratigraphique des occupations humaines du gisement du Petit Saule (Bahain et al., 1996 ; Locht et al., 1997). La relative pauvreté du bilan pédosédimentaire au niveau du site paléolithique, par comparaison avec la séquence beaucoup plus complète du Chamesson, n'a pas tout de suite permis de lever un certain nombre d'incertitudes concernant la position des artefacts dans l'histoire pédosédimentaire locale et leur interprétation chronostratigraphique.

Ce travail a cependant pu être complété par l'étude du profil de Chauvry, situé à $1 \mathrm{~km}$ à l'est - sud-est de celui du Chamesson (fig. 3). Cette nouvelle séquence a permis de mettre en évidence l'extension de la couverture limoneuse vers le sud-est, de compléter l'étude de la stratigraphie du Weichsélien moyen, particulièrement bien représenté à cet endroit, et de consolider les interprétations pédostratigraphiques concernant cette partie de la séquence.

Plus récemment, l'étude des profils dégagés lors de la deuxième phase de terrassement en mars 1999 a permis de compléter ces recherches (profil VA-99, fig. 6). Cette séquence, située en position intermédiaire entre les profils du Chamesson et du Petit Saule, est exceptionnellement complète dans sa partie basale, attribuable au Début Glaciaire weichsélien et a permis de consolider l'interprétation stratigraphique du gisement paléolithique.

\section{STRATÉGIE D'ÉCHANTILLONNAGE}

Un levé détaillé au $1 / 20$, dans lequel sont localisés tous les échantillons, a été effectué pour chaque profil (fig. 8, 9 et 10). L'échantillonnage systématique de chaque séquence a été réalisé pour les différentes études suivantes (fig. 8, 9 et 10).
Susceptibilité magnétique : prise d'échantillons cubiques de $2 \mathrm{~cm}$ d'arête tous les $5 \mathrm{~cm}$ pour mesure en laboratoire avec un susceptibilimètre Bartington MS2 (analyses réalisées par D.-D. Rousseau).

Sédimentologie: prélèvement tous les $10 \mathrm{~cm}$ au moyen d'un carottier de $4 \mathrm{~cm}$ de diamètre. Sur ces échantillons ont été effectuées une granulométrie 6 fractions sans décarbonatation par tamisage et pipette $(<1 \mu \mathrm{m} ; 1-2 \mu \mathrm{m}$; 2-20 $\mu \mathrm{m} ; 20-50 \mu \mathrm{m} ; 50-200 \mu \mathrm{m}$ et 200-2 $000 \mu \mathrm{m})$ et une calcimétrie (Laboratoire d'analyse des sols, INRA-Arras, et Laboratoire de géomorphologie, université de Lille).

Géochimie : analyse du fer total (après mise en solution totale dans de l'acide fluorhydrique et mesure par absorption atomique de flamme) et du carbone organique total (COT) (méthode sulfochromique) : échantillonnage tous les $10 \mathrm{~cm}$ (Laboratoire d'analyse des sols INRA-Arras).

Carbone organique et $\partial^{13} \mathrm{C}$ de la matière organique (analyse élémentaire et spectrométrie de masse isotopique) : maille de prélèvement de $5 \mathrm{~cm}$ (C. Hatté).

Malacologie: tests ponctuels et échantillonnage en colonne continue tous les $10 \mathrm{~cm} ; 10$ à $15 \mathrm{~kg}$ par échantillon (N. Limondin-Lozouet).

Palynologie: tests ponctuels et échantillonnage en colonnes tous les $5 \mathrm{~cm}$ (A. Gauthier).

Micromorphologie: des séries de blocs non perturbés ont été prélevées dans l'ensemble des profils (P. Antoine, P. Haesaerts, H. Metsdagh)

Datation TL-OSL sur sédiment : les échantillons destinés à la datation TL-IRSL ont été prélevés par carottage horizontal (diamètre : $10 \mathrm{~cm}$ ) dans les différents profils. La dosimétrie a été effectuée par spectrométrie gamma en laboratoire sur des échantillons en sacs prélevés en parallèle. (M. Frechen).

Datation TL sur silex: des silex chauffés du site du Petit Saule ont été choisis parmi les objets recueillis lors de la fouille. La dosimétrie a été mesurée directement sur le site par spectrométrie gamma portable dans des forages à la tarière. (N. Debenham).

\section{DESCRIPTION DES UNITÉS LITHOSTRATIGRAPHIQUES}

Le relevé systématique des différents profils dégagés lors de la fouille et plusieurs sondages profonds ont permis de reconnaître la stratigraphie du gisement (tabl. I). Cette démarche débouche sur la mise en 

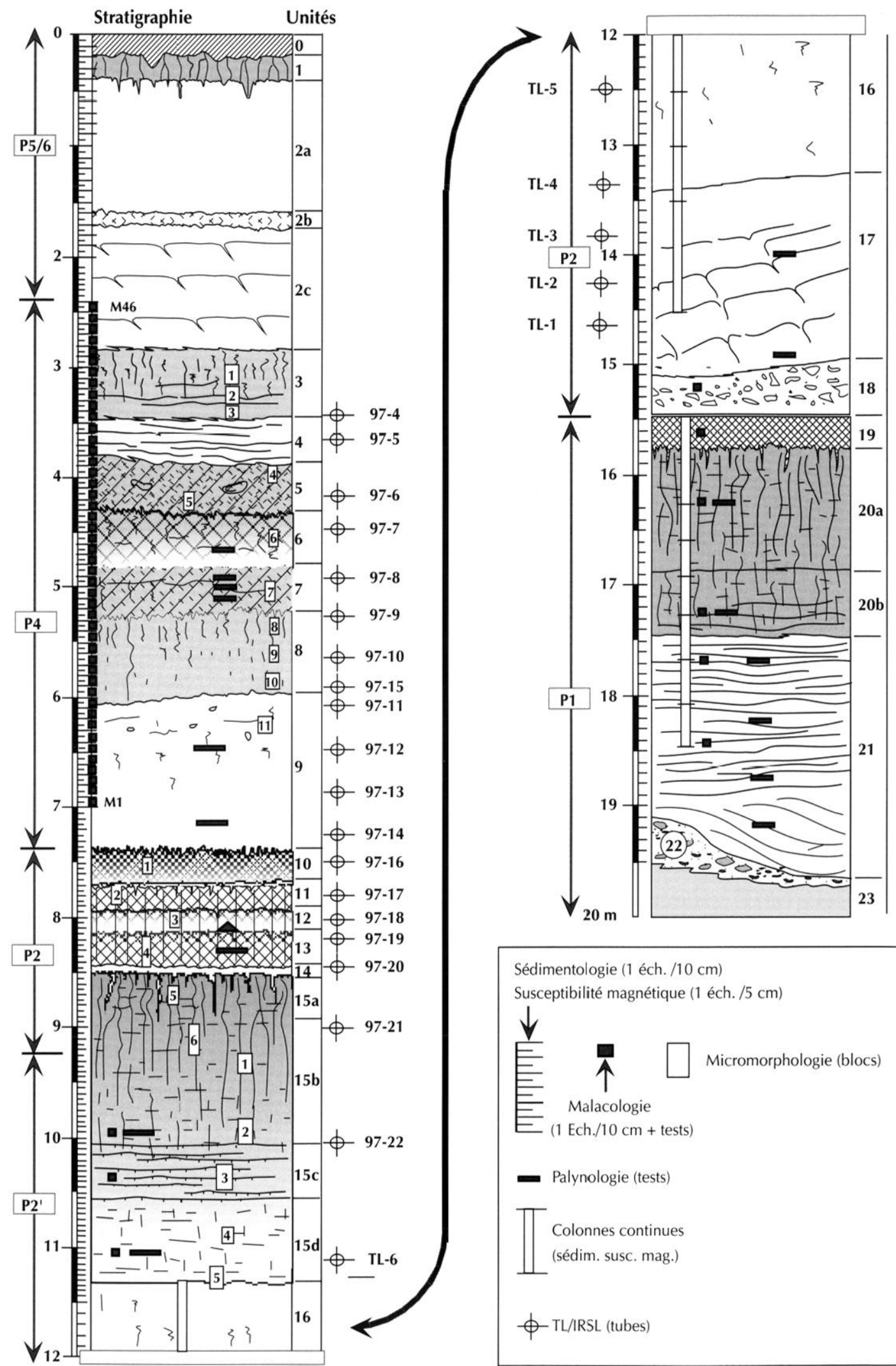

Sédimentologie $(1$ éch. $/ 10 \mathrm{~cm})$
Susceptibilité magnétique $(1$ éch. $/ 5 \mathrm{~cm})$

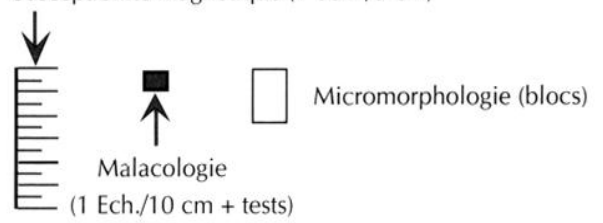

- Palynologie (tests)

TColonnes continues (sédim. susc. mag.)

$\oint$ TL/IRSL (tubes)

Fig. 8 - Villiers-Adam, Le Chamesson : séquence synthétique PI-P6 et localisation des échantillons (description des unités, voir tabl. I), dessin P. Antoine, J.J. Bahain, A. Gauthier, J.-L. Locht, N. Limondin. 

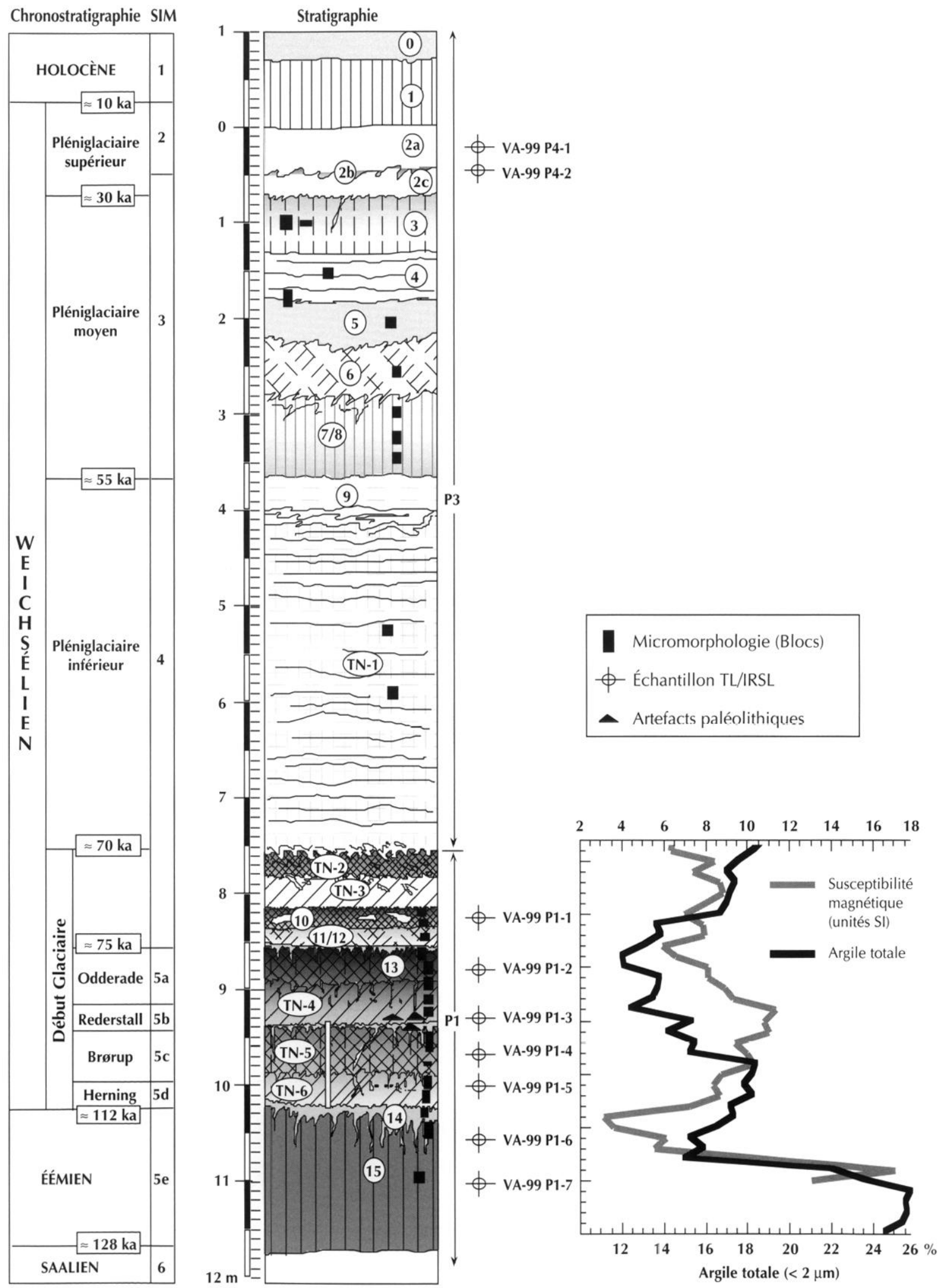

Fig. 9 - Villiers-Adam coupe 99 (VA-99) : séquence synthétique PI-P3 et données sédimentologiques P1 (description des unités, voir tabl. I), dessin P. Antoine et J.-J. Bahain. 


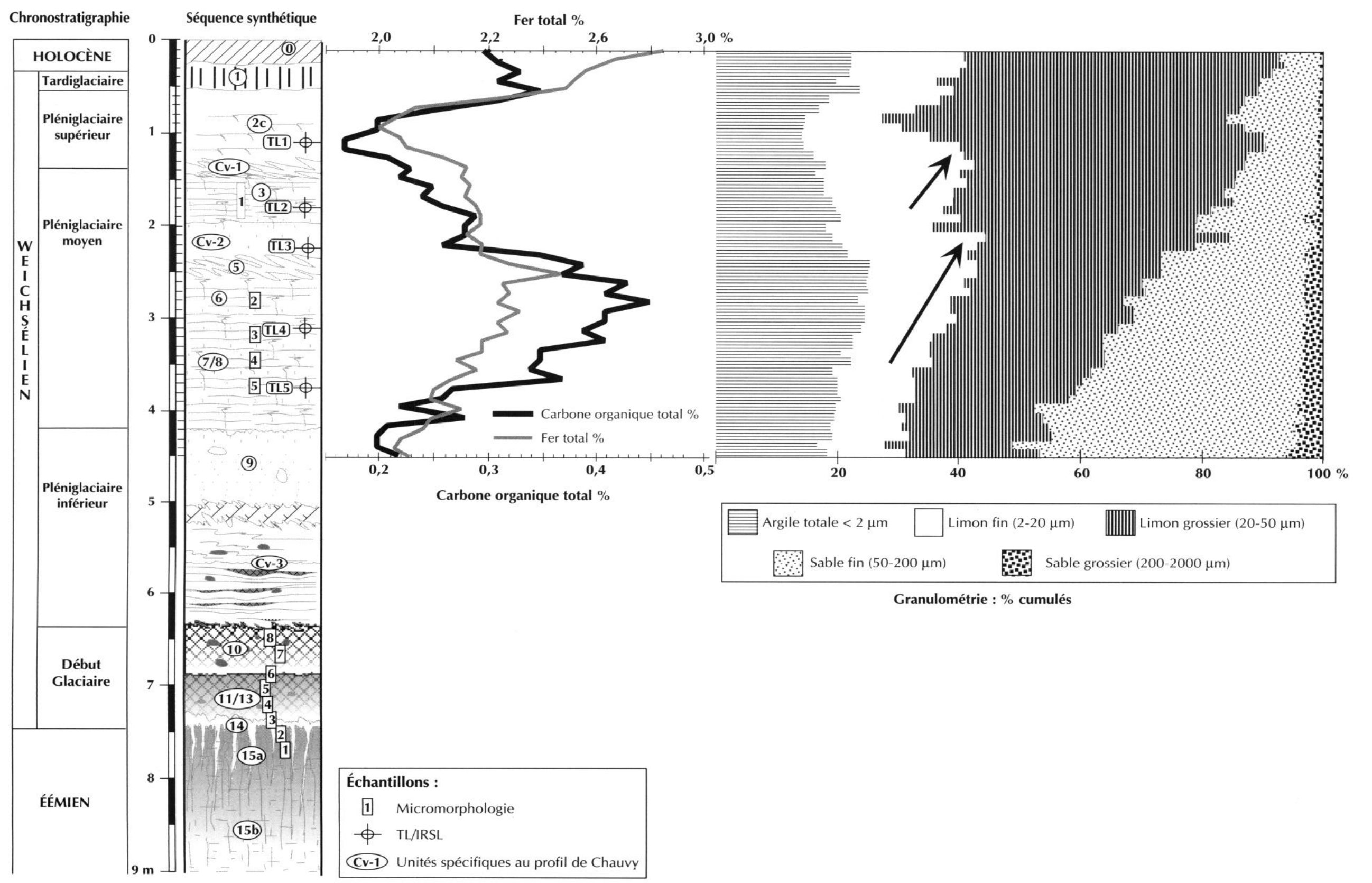

Fig. 10 - Chauvry : levé détaillé, localisation des échantillons et données sédimentologiques pour la partie supérieure du Pléniglaciaire moyen (description des unités, voir tabl. I), dessin P. Antoine. 
Tabl. I - Description et interprétation pédosédimentaire des unités observées dans les profils du ('hamesson ( $n^{\prime \prime} 0$ à 23), de Chaurry (Cv-1 à Cv-3), de VA-99 (TN-1 à TN-6) et de la fouille du Petit Saule (PSF) à Villiers-Adam (fig. 6 à 8 et 10 à 13).

Micromorphologie, abondance relative des traits pédologiques : - -, rares ; +, quelques ; ++, nombreux/importants ; +++ , très nombreux/abondants.

\begin{tabular}{|l}
\hline \multicolumn{1}{|c|}{ Unités et description (macro-, méso- et microscopiques) } \\
\hline 0 - Limon brun gris à silex épars. \\
\hline $\mathbf{1}$ - Limon argileux brun orangé, non calcaire, compact, avec nombreuses bioturbations, \\
agrégats, revêtements argileux et structure prismatique diffuse. \\
\hline 2a - Limon calcaire (15-17 \%) sableux homogène, brun jaune (10YR 6/6 6/4), à nombreux bio- \\
tubules à enduit calcaire et traces de racines provenant des horizons de sol sus-jacents. \\
2b - Petit horizon à langues hydromorphes grisâtre (VA-99). \\
2c - Limon sableux calcaire brun jaune clair finement lité à niveaux de microfentes emboîtés \\
(VA-99). \\
2d - Limon calcaire homogène, brun jaune clair (VA-99), limite inférieure avec traces \\
d'érosion localement très marquées (10YR 6/6). \\
\hline Cv-1 - Petit horizon à langues hydromorphes grisâtre (Chauvry), localement assez épais dans \\
le profil VA-99, où il se développe au sommet de 3.
\end{tabular}

3 - Limon brun clair à brun orangé clair (7,5 YR 5/8), faiblement calcaire $\left(\mathrm{CaCO}_{3}: 10-12 \%\right)$, argileux $(<2 \mu \mathrm{m}: 18-20 \%)$, avec bandes et plages plus grises diffuses, porosité racinaire bien développée avec enduits calcaires (hypocoatings).

Traces de litage sédimentaire ++ , biosphérö̈des de calcite (vers de terre + , bioturbation et déjections ++ .

4 - Limon sableux finement lité à lits de sable calcaire plus clairs, (2-5 mm), lits de nodules ferromanganiques $(\mathrm{Fe}-\mathrm{Mn})$ remaniés, traces de cryoturbations. Limite inférieure fortement érosive avec localement structures en chenaux concentrés.

Traces de litage sédimentaire avec granoclassement +++ , pas de biosphéroïdes, carbonates secondaires -

Cv-2 - Limon calcaire sableux homogène gris jaune à structure finement feuilletée (Chauvry).

5 - Limon lœssique calcaire $\left(\mathrm{CaCO}_{3}: 20 \%\right)$, homogène, gris clair à gris jaune clair, avec nombreux tubules à enduits calcaires, grosses bioturbations ovalaires $(\varnothing: 6-10 \mathrm{~cm})$, taches d'oxydation et nodules Fe-Mn épars.

Nodules Fe-Mn et Fe-Mn dendritique ++, biosphéroïdes +++, micronodules argilo-humiques (déjections, $\varnothing: 1-1,5 \mathrm{~mm}$ ) ++ , hypocoatings et cellules calcitées ++ , souvent fracturés par le gel, structure lamellaire millimétrique (gel-dégel).

6 - Limon sableux à sablo-argileux $(<2 \mu \mathrm{m}: 23-25 \%$ ), brun grisâtre (10YR 5/6 5/8), (faiblement humifère) calcaire, à structure granulaire, nombreux biotubules à revêtements calcaires, petits nodules Fe-Mn abondants.

Microstructure : idem $n^{\circ} 7$, avec structure de gel-dégel millimétrique plus nette.

7 - Limon sablo-argileux $(<2 \mu \mathrm{m}: 23 \%)$, brun grisâtre humifère (COT : 0,35-0,4\%), nombreux biosphéroïdes de calcite $(\varnothing: \approx 500 \mu \mathrm{m})$, limite inférieure diffuse sur $10 \mathrm{~cm}$.

Hypocoatings et cellules racinaires calcitées +++ , biosphérö̈des ++ , nodules $\mathrm{Fe}-\mathrm{Mn}++$, microagrégats limono-argileux (déjections ++ , apparition d'une structure lamellaire fine (gel-dégel).

8 - Limon sablo-argileux $\left(<2 \mu \mathrm{m}\right.$ : 18-20\%), non calcaire (excepté le $\mathrm{CaCO}_{3}$ secondaire), brun à brun orangé sombre (10YR 4/6), plus compact que les niveaux sous et sus-jacents, avec structure granulaire à finement feuilletée (polyédrique fine), nombreux tubules à revêtements calcaires, biopores très abondants, traces d'activité de vers, et nombreux petits nodules Fe-Mn épars, nombreuses " taches " noires irrégulières (manganèse) dans la partie inférieure, limite inférieure très nette (décarbonatation).

Accumulations silto-argileuses dans la matrice, localement autour des biopores ++ , hypocoatings épais +++ et cellules racinaires calcitées ++ , biosphéroïdes de calcite + (apparition au sommet de 8), micronodules limono-argileux (déjections) +, nodules Fe-Mn ++.

9 - Sable moyen calcaire (8-10\%), jaune clair, passant vers le haut à un sable limoneux calcaire homogène à pseudomycélium et enduits calcaires abondants (recarbonatation). Nombreuses bioturbations $(1-2 \mathrm{~cm})$ à remplissage sablo-argileux dans la partie supérieure. Contact de base avec grandes figures d'érosion concentrées.

Nodules Fe-Mn ++ et imprégnation Fe-Mn dendritique +, hypocoatings épais, cellules racinaires calcitées +++ , plus ou moins intégrées à la matrice par la bioturbation, pas de biosphéroïdes de calcite.

TN-1 et Cv-3 - Ensemble de sables et limons lités (litages irréguliers centimétriques à pluricentimétriques), avec nombreuses petites failles synsédimentaires, blocs limoneux, lits de nodules Fe-Mn et localement lentilles de sol humifère remaniées ( $\mathrm{Cv}-3)$.

TN-2 - Limon sableux brun sombre humifère, à structure lamellaire fine et nombreux pédotubules à enduits calcaires, contact supérieur fortement bioturbé.

Lœss calcaire typique.

(b) Horizon hydromorphe à langues de gélifluxion (gelic gleysol).

(c) Lœss calcaire lité (nivéo-éolien), à microfentes de cryo-dessication.

(d) Lœss calcaire typique avec figures d'incision localisées.

Petit gley géliflué (gelic gleysol), avec localement fentes de gel plus ou moins retouchées par des processus thermokarstiques en VA-99.

Petit horizon $\left(\mathrm{B}_{\mathrm{w}}\right)$ de type brun arctique plus ou moins géliflué (gelic cambisol), affecté par le gel-dégel et localement fortement érodé en VA-99 (thermokarst sur anciennes fentes à coins de glace, profil P4).

Dépôts sablo-limoneux ruisselés remaniant des éléments de sols sous-jacents, avec localement fentes de gel, figures d'érosion intense à la base.

Lœss calcaire sableux.

Gley de toundra légèrement humifère (COT : $0,38 \%$ ), avec structure de gel-dégel très marquée (permafrost, gelic gleysol).

Horizon faiblement humifère du type " sol de type prairie arctique " $(\approx 0,3 \%$ COT) affecté par un geldégel intense.

Horizon supérieur du sol 8 de type brun arctique (gelic cambisol) affecté par un gel-dégel très net.

Horizon $\left(\mathrm{B}_{\mathrm{w}}\right)$ de type brun arctique à brun boréal développé sur limon sableux préalablement décarbonaté (gelic cambisol).

Sédiment éolien avec, à la base, de grandes figures d'érosion d'origine thermokarstique développées à partir de grandes fentes à coins de glace.

Colluvions limono-sableuses mises en place par ruissellement avec processus nivéo-éoliens et gélifluxion dans un contexte de type périglaciaire (failles, blocs gelés), très forte érosion à la base.

Petit horizon humifère de type steppique sur limon lœssique sableux d'origine éolienne. 
Tabl. I - (suite).

Unités et description (macro-, méso- et microscopiques)

TN-3 - Limon brun gris moins humifère que TN-2, homogène, sablo-argileux avec très nombreuses bioturbations à remplissage humifère (10).

10 - Limon non calcaire brun sombre, humifère $(0,3 \%)$, homogène à porosité fine abondante et nombreux tubules à enduits calcaires.

Papules (fragments de revêtements argileux $<50 \mu \mathrm{m})+$, microgranules organiques +++ nodules Fe-Mn $\leq 1 \mathrm{~mm}$ remaniés +++ , pédotubules (sans remplissage $\approx 1 \mathrm{~mm}$ ) +++ , imprégnation ferromanganique $(\mathrm{Fe}-\mathrm{Mn})++$, manchons racinaires à imprégnation calcaire (hypocoatings) ++ .

11 - Limon argileux non calcaire brun gris, à structure polyédrique à lamellaire moyenne (ép 2-5 mm), avec fins squeletanes dans la partie inférieure, et nodules Fe-Mn épars. Partie supérieure en partie remaniée par frost-creep et affectée par une hydromorphie peu marquée. Limite inférieure irrégulière (bioturbation) avec quelques cailloux épars.

Revêtements argilo-humiques à silto-humiques +, striotubules illuviés +, papules argileuses (> $50 \mu \mathrm{m})++$ et nodules Fe-Mn remaniés +++, microcharbons et débris végétaux humifiés +++ , calcite racinaire post-illuviation + , imprégnation ferromanganique ++ , nodules Fe-Mn zoné -.

12 - Limon argileux non calcaire gris brun clair à taches grises et traces d'oxydation éparses, structure polyédrique à lamellaire moyenne, nombreux nodules Fe-Mn, nodules remaniés provenant de l'horizon sous-jacent 13, coloration plus claire que les niveaux 11 et 13 . Limite inférieure bioturbée avec glosses et silex épars.

Revêtements argilo-humiques à silto-humiques stratifiés ++ , localement papulisés et fracturés par le gel ; striotubules (ø: $4-5 \mathrm{~mm}$ ) à remplissage argilo-humique stratifié ++ , striotubules et chambres $(\varnothing: 10 \mathrm{~mm})$ à remplissage silteux finement stratifié + , colmatage silteux clair final, bioturbation (vers, insectes) ++, imprégnation Fe-Mn +++ et nodules Fe-Mn zonés, micronodules et enduits ferriques noir $(<50 \mu \mathrm{m})$ (bactéries ?).

13 - Limon argileux $(<2 \mu \mathrm{m}: 27-28 \%)$ non calcaire brun noir (10 YR 4/3 3/3), à très forte structure prismatique centimétrique puis polyédrique en profondeur soulignée par des épais enduits de silts blancs (squeletanes). Biopores abondants avec illuviations brun noir argilohumique épaisses $(\approx 1 \mathrm{~mm}$ ), et enduits de silt blanc. Présence de logettes d'hibernation de vers (I 1-1,5 cm) avec pellets argileux ovoïdes (1-3 mm) et colmatage silteux. Limite inférieure très irrégulière (glosses grisâtres) et nombreuses traces de racines à remplissage brun noir.

Traces de litages sédimentaires (VA-99) +, revêtements argilo-humiques à silto-humiques stratifiés épais +++ souvent papulisés et fortement fracturés par le gel, striotubules à illuviation stratifiée ++, nodules Fe-Mn zonés +, imprégnation ferrique +, microcharbons de bois (< $500 \mu \mathrm{m})$, colmatages silteux (squeletanes) dans les pédotubules et chambres biologiques +++ , imprégnation calcaire périracinaire (micrite).

TN-4 - Limon argileux compact brun grisâtre (10YR 4/4) à taches grises (silts lavés) et sombres (bioturbations à remplissage humifère), avec très forte imprégnation de la bioporosité par du silt blanc lavé, nombreuses logettes colmatées, illuviations argilo-humiques brun noir sur la porosité, porosité racinaire plus faible qu'en 13, structure polyédrique à lamellaire grossière (1-1,5 cm). Le contact avec 13, extrêmement irrégulier, est jalonné par des nombreuses traces de racines à remplissage plus humifère.

Des pièces lithiques non patinées que I'on peut rapprocher de celles du Petit Saule ont été découvertes à la base de cette unité près du contact avec TN-5.

Revêtements argilo-humiques à silto-humiques stratifiés épais +++(plus développés qu'en 13), fortement fracturés par le gel et la bioturbation, revêtements brun foncé non laminés, non biréfringents avec micropoints noirs + , microcharbons et débris ligneux humifiés $(\leq 500 \mu m)++$, nodules Fe-Mn ++, nodules zonés développés autour d'un microcharbon + , imprégnation finale (enduits et micronodules noirs) post-illuviation ++ , pédotubules (ø : 4-6 mm) et chambres abondants avec colmatage silteux.

TN-5 - Limon argileux $(<2 \mu \mathrm{m}: 18 \%)$ brun (10YR 4/4), non calcaire, très compact, avec nombreuses taches de déferrification, nodules Fe-Mn abondants, illuviations d'argile brun sombre à brun rouge, et nombreuses logettes d'hibernation de vers $(\varnothing: 1-2 \mathrm{~cm})$. Cette unité est séparée de I'horizon sus-jacent par un niveau de glosses grisâtres irrégulières de 20 à $60 \mathrm{~cm}$.

Revêtements argilo-humiques à silto-humiques stratifiés épais +++ (plus développés qu'en $T \mathrm{~N}-4)$, fortement fracturés par le gel et la bioturbation, revêtements argilo-humiques sur fissures de gel subhorizontales, microcharbons et débris ligneux humifiés $(\leq 500 \mu \mathrm{m})++$.

TN-6 - Limon brun gris compact à très nombreux nodules Fe-Mn et plages oxydées oranges Horizon inférieur $\mathrm{B}_{\text {th }}$ profond du sol de I'unité TN-4 avec niveau diffus de charbons de bois dans la partie médiane, logettes et bioturbations plus développé sur colluvions limoneuses.

rares qu'en TN-4 (cf. unité $\mathbf{C v - 1 )}$.

Revêtements argilo-humiques à silto-humiques ++ , revêtements argilo-humiques sur fissures

de gel subhorizontale + , microcharbons et débris ligneux humifiés $(\leq 500 \mu \mathrm{m})+++$
Colluvions limono-argileuses en partie remaniées par frost-creep avec développement pédologique très peu marqué de type sol gris-forestier (phase finale du complexe SGF 11/12/13) du Chamesson. Cependant le matériel sédimentaire plus homogène qu'en 12 indique probablement un début d'apport éolien.

Horizon $B_{h}$ de gris-forestier (Greyzem) géliflué, faiblement développé sur colluvions limono-argileuses remaniant 13, avec phase d'hydromorphie et de gel profond et phase finale d'illuviation silteuse très marquée (bioporosité colmatée).

Partie supérieure du sol 13 dégradée par les phénomènes de gel-dégel.

Horizon $B_{\text {th }}$ tronqué de sol humifère de type grisforestier (Greyzem) fortement structuré, développé sur colluvions sablo-argileuses litées, avec très forte structure de gel profond, hydromorphie et colmatage terminal de la porosité par des silts de lavage (fonte de la couverture nivale).

Colluvions limono-sableuses alimentées par le remaniement des sols sous-jacents (nodules argileuxi, avec très importante bioturbation et traces d'illuviation argilo-humiques en provenance du $B_{\text {th }} 13$, phase de gel saisonnier et imprégnation silteuse finale (horizon profond du sol 13).

Horizons $\mathrm{B}_{t}$ à $\mathrm{B}_{\mathrm{t}}$ faiblement humifères sur colluvions argileuses, avec gel saisonnier profond très marqué et importante hydromorphie terminale (Greyzem/ Luvisol). 
Tabl. I - (fin)

Unités et description (macro-, méso- et microscopiques)

14 - Petit horizon limoneux grisâtre visible au contact entre 13 et 15a remplissant les nom- Horizon de dégradation glossique et érosion.

breuses glosses qui jalonnent le contact supérieur du $\mathrm{B}_{\mathrm{t}} 15 \mathrm{a}$; présence de quelques cailloux épars. Dans la coupe VA-99, cette unité s'épaissit (15-20 cm) et se caractérise par l'abondance des glosses et la présence d'auréoles orangées d'oxydation sur les traces de racines (anneaux de Liesegang).

15a - Limon argileux compact $(<2 \mu \mathrm{m}: 25-30 \%)$, brun orangé (traces d'oxydation) à structure plus nettement prismatique, très nombreuses bioturbations à remplissage de limon argilo-humique brun noir (13), glosses grisâtres et nombreux nodules Fe- $\mathrm{Mn}$ millimétriques.

Revêtements argileux :

Phase 1 : revêtements argileux jaunes hyalins microlités souvent remaniés (ferriargilanes) + ; Phase 2 : brun rouge, argilo-humiques à silto-humiques stratifiés en place +++ , souven fortement papulisés (bioturbation +++ ) ;

Enduits et micronodules noirs (Fe-Mn ?) post-illuviations de la phase $2+$, colmatage silteux de la porosité +++ (post phase 2).

15b - Limon argileux $(<2 \mu \mathrm{m}: 20-25 \%)$, non calcaire, brun rouge $(7,5$ Y $4 / 4-4 / 6$ en VA-99), compact, à structure polyédrique à prismatique centimétrique ; nombreuses traces d'activité des vers (tubules et logettes) et biopores millimétriques abondants plages grisâtres hydromorphes centimétriques. Illuviation d'argile en deux phases : illuviation d'argile brune puis illuviation argilo-humique brun sombre épaisse essentiellement représentée sur les traces de galeries et biopores. Présence de revêtements de silts blancs dans les biopores. Limite inférieure graduelle sur $10 \mathrm{~cm}$.

15c - Limon faiblement argileux $(<2 \mu \mathrm{m}: 15-18 \%)$, non calcaire, brun à bandes grisâtres Horizon à doublets du sol 15a/b. millimétriques irrégulières (faciès Limon à doublets). L'ensemble possède une structure lamellaire millimétrique très marquée (ép. 2-5 mm) et contient de très nombreux petits nodules Fe-Mn.

15d - Limon non calcaire $(<2 \mu \mathrm{m}: 15-16 \%)$, moins compact que les niveaux sus-jacents avec Horizon $\mathrm{B}_{\mathrm{t}}\left(\mathrm{B}_{3 \mathrm{f}}\right)$ du sol 15a/b.

porosité fine moins abondante, illuviation brune sur les biopores ( $\hat{\mathrm{U}} 1 \mathrm{~mm})$, bandes plus argileuses diffuses très nombreux petits nodules Fe-Mn. Limite inférieure abrupte (décarbonatation). Ce niveau est traversé par de fines fentes (ép. 0,5-2 cm) colmatées par des enduits d'argile humique brun noir et de silts blancs en relation avec les illuviations et les phases de gel observées dans le sol gris-forestier de l'unité 13.

16 - Limon calcaire (11-14\%), homogène brun jaune clair, à rares lits sableux, fins nodules Lœss calcaire typique.

Fe-Mn épars, biopores et biogalleries avec carbonates secondaires. Limite inférieure graduelle sur $20 \mathrm{~cm}$.

17 - Limon calcaire (11-14\%), brun jaune clair, finement lité à lits sableux et lits brunâtres millimétriques, avec nombreuses microfentes emboîtées. Limite inférieure abrupte et érosive (contact avec le cailloutis 18).

18 - Ensemble grossièrement lité à niveaux de cailloutis et lits sableux, limite inférieure abrupte (érosion).

19 - Limon sableux (100-200 $\mu \mathrm{m}$ : 40-45\%), gris brunâtre faiblement humifère, à structure granulaire et nodules Fe-Mn épars.

20a - Limon sablo-argileux $(<2 \mu \mathrm{m}: 24-25 \%)$, brun à brun orangé, non calcaire, compact, à structure lamellaire de plus en plus nette vers la base et taches d'hydromorphie grisâtres éparses, nodules Fe-Mn assez abondants.

20b - Limon sablo-argileux $(<2 \mu \mathrm{m}: 24-25 \%)$, brun orangé, non calcaire, compact, à structure polyédrique diffuse, avec quelques litages grossiers apparents.

21 - Sables jaune à gris-jaune lités à litages épais et réguliers $(2-5 \mathrm{~cm})$, avec illuviations argileuses brunes dans la partie supérieure.

22 - Cailloutis hétérométrique constitué par une accumulation de blocs de meulière emballés dans une matrice sablo-argileuse brun-rouge.

\section{3 - Sables homogènes non calcaires gris clairs.}

PSF (Fouille) - Limon argileux non calcaire à structure polyédrique à granulaire, (unité contenant le niveau paléolithique du Petit Saule), à nombreuses taches grisâtres (racines et bioturbations en provenance de 13), nodules de sol remaniés, nombreux nodules Fe-Mn et localement de nombreux charbons de bois épars (1-5 mm).

Revêtements argilo-humiques à silto-humiques stratifiés souvent fissurés par le gel ++, papule argilo-humiques +++ , cavités et chambres $(\varnothing: 1-1,5 \mathrm{~cm})$ et biotubules $(\varnothing: 3-4 \mathrm{~mm})$ à remplis sage silteux stratifié + , microcharbons + , débris végétaux humifiés + , nodules $\mathrm{Fe} \mathrm{Mn}$ remaniés ++ , hypocoatings ++ . Structure polyédique $(3-4 \mathrm{~mm})$ localement très marquée.
Horizon $B_{t}$ profond $\left(B_{2 t}\right)$, tronqué de sol brun lessivé Luvisol), polyphasé avec nombreux traits hérités d'une pédogenèse de type sol gris-forestier $\left(\mathrm{B}_{\mathrm{th}}\right)$, avec importante phase de gel profond et phase d'hydromorphie terminale.

L'horizon 15a du Chamesson montre en fait la surimposition des traits pédologiques correspondant à la succession des horizons 15 à 13 individualisés en VA-99.

Horizon $\mathrm{B}_{1}$ profond $\left(\mathrm{B}_{2 \mathrm{l}}\right)$ de sol brun lessivé (Luvisol), polyphasé avec illuviations argilo-humiques liées au développement de $15 \mathrm{a}$, importante phase de gel saisonnier et phase d'hydromorphie terminale. 


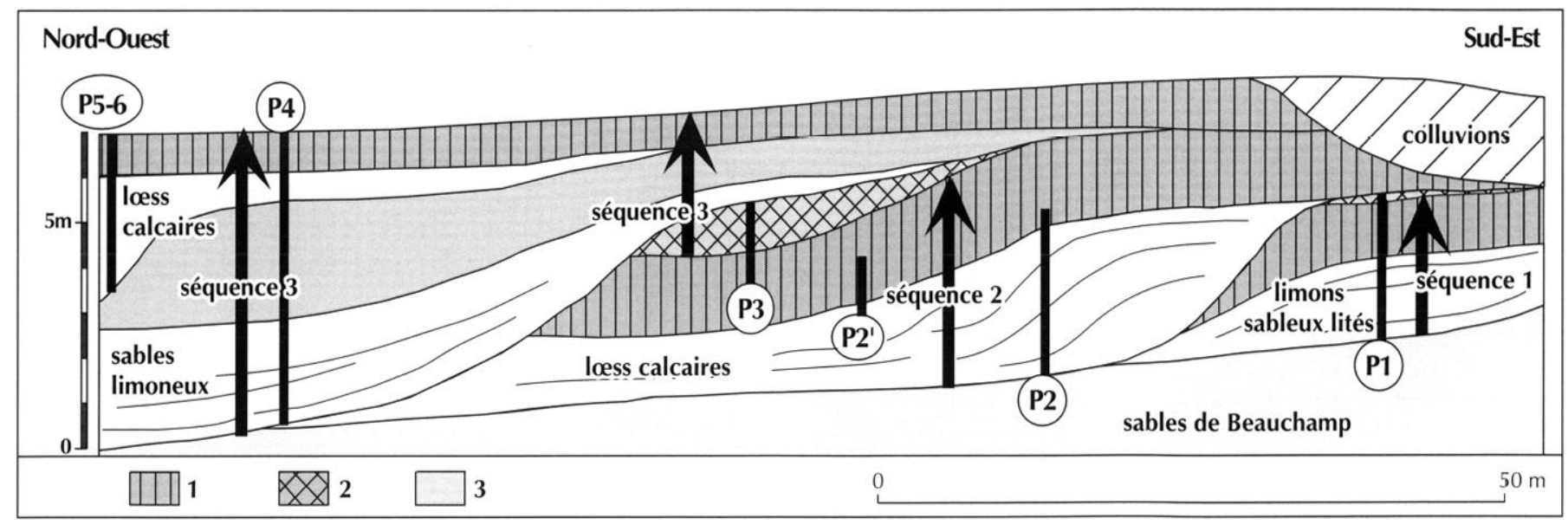

Fig. 11 - Morphostratigraphie générale simplifiée du secteur du Chamesson et enchaînement des séquences pédosédimentaires : 1, horizons pédologiques de type $B_{t}$ de sol brun lessivé ; 2 , horizons ou complexes d'horizons de sols humifères; 3, complexe pédosédimentaire à sols bruns interstadiaires (Arctique-Boréal), dessin P. Antoine.

évidence d'une séquence initiale complexe de 22 unités au niveau du Chamesson (numérotation continue 0 à 22) reposant sur le substratum tertiaire (unité 23). Le substratum local est représenté par des sables blancs azoïques d'âge éocène (sables de Beauchamp, Auversien, Bartonien supérieur).

Le profil synthétique de Villiers-Adam (fig. 8) est construit sur la base de la corrélation des séquences P1 à P6 (fig. 11) par l'intermédiaire de nombreux horizons repères, notamment pédologiques, visibles dans tous les profils comme par exemple les unités 15, 10 et 7-8. Au sein de cette série de référence, des unités spécifiques, observées uniquement dans les profils VA-99 (Talus nord), et dans les coupes du Petit Saule et de Chauvry, ont été ajoutées. Elles sont alors affectées d'un indice faisant référence à leur localisation dans ces profils (respectivement TN-1 à TN-6, PSF et Cv-1 à $\mathrm{Cv}$-3).

La description de l'unité PSF est présentée à la fin du tableau I, en raison de son importance pour l'interprétation du site paléolithique et du fait que sa position dans la stratigraphie globale de Villiers-Adam est établie sur la base des corrélations que l'on peut effectuer avec les autres profils. En effet, l'analyse détaillée des différents profils, complétée par les données micromorphologiques, montre que l'unité PSF est clairement antérieure au développement du sol 13, mais postérieure au $\mathrm{B}_{\mathrm{t}}$ complexe 15 (15a). Par ailleurs, compte tenu de la position des pièces lithiques découvertes en VA-99 (fig. 9), l'unité PSF est logiquement postérieure aux unités TN-4 et TN-5. Il est donc proposé de la mettre en parallèle avec la base de l'unité TN-4 qui possède par ailleurs des caractéristiques sédimentaires et pédologiques très proches (tabl. I).

\section{STRATIGRAPHIE ET MORPHOSTRATIGRAPHIE GÉNÉRALE}

L'analyse stratigraphique des différents profils de Villiers-Adam permet de mettre en évidence une séquence complexe dont l'épaisseur cumulée atteint environ $19 \mathrm{~m}$ (fig. 8). Cette formation pléistocène (formation de Villiers-Adam) repose sur le substrat tertiaire (sables de Beauchamp) par l'intermédiaire d'un contact érosif à la base de l'unité 22, qui correspond à un cailloutis à blocs de meulière. La stratigraphie de la partie supérieure de la formation est complétée à moyenne et longue distances par les séquences de VA-99 (épaisseur cumulée: $12 \mathrm{~m}$ ), du Petit Saule (épaisseur cumulée : $4 \mathrm{~m}$ ) et de Chauvry (épaisseur cumulée : $9 \mathrm{~m}$ ).

Au sein de la formation de Villiers-Adam, l'analyse de la stratigraphique et de la géométrie des différentes unités permet de définir une succession de trois grandes séquences climatosédimentaires partiellement emboîtées sur le versant (fig. 11).

\section{SÉQUENCE 1}

La séquence 1 est uniquement visible à la base des coupes du Chamesson (unités 21 à 19), où elle est 


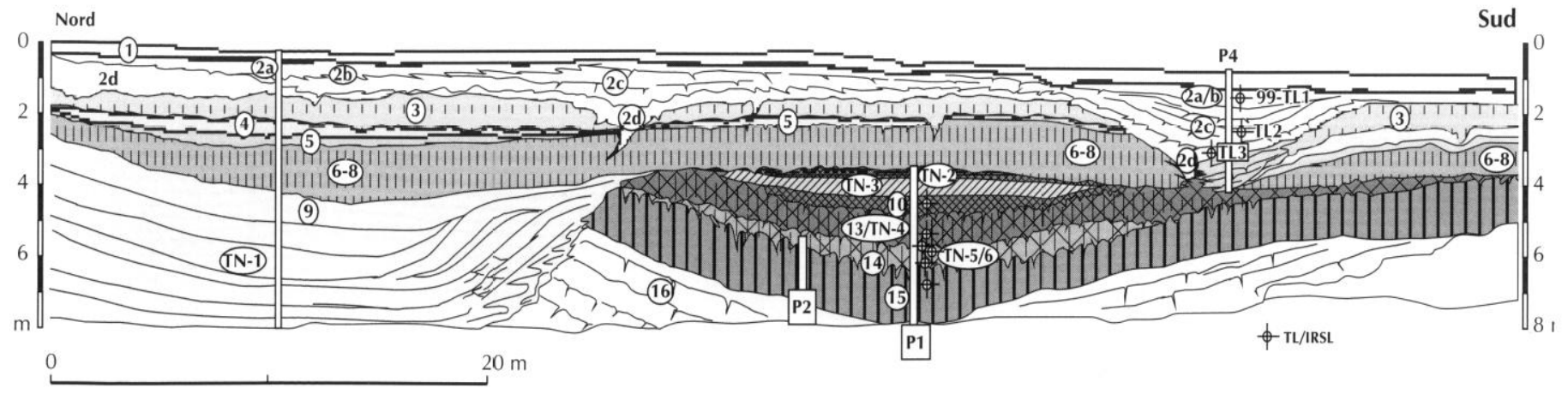

Fig. 12 - Villiers-Adam, coupe 99 (VA-99) : Stratigraphie générale et localisation des profils détaillés (description des unités, voir tabl. I), dessin P. Antoine, J.J. Bahain et J.-L. Locht.

préservée à la faveur d'une dépression dans les sables tertiaires localisée dans la partie ouest du site du Chamesson (profil P1, fig. 9). Elle débute par la mise en place de sables limoneux lités (21) et se termine par la formation d'un sol brun lessivé complexe de rang interglaciaire (20a et $20 \mathrm{~b}$ ).

\section{SÉQUENCE 2}

La séquence 2, dont l'épaisseur maximale atteint environ 7,5 $\mathrm{m}$ dans la partie ouest du Chamesson, débute par un petit sol humifère grisâtre (unité 19 ; fig. 8), suivi par une phase d'érosion intense qui se marque par la création d'un vallon subparallèle à la pente du versant (incision de 3 à $4 \mathrm{~m}$ de profondeur par rapport au sommet de la séquence précédente: fig. 11). Cette structure, jalonnée par un cailloutis (18), est ensuite comblée par des lœess calcaires lités, puis des loess homogènes au sommet desquels se développe un sol brun lessivé complexe (unités 15d à 15a).

\section{SÉQUENCE 3}

La séquence 3, dont l'épaisseur cumulée atteint 7,5 m (Chamesson) à $10 \mathrm{~m}$ (VA-99), est la plus complexe de la formation de Villiers-Adam. Elle débute dans tous les profils par une érosion du sommet de l'horizon $B_{t}$ interglaciaire 15 (15a-15d), suivie par la mise en place des unités suivantes (fig. 8, 9, 11 et 12) :

- complexe de sols humifères (unités 13 à 10 du Chamesson), particulièrement développé dans le profil VA-99 (2,7 m, unités TN-6 à TN-2) ;

- limons sableux lités, puis homogènes (unités TN-1 et 9$)$;
- complexe de sols bruns et gley de toundra (unités 8 à 3) ;

- lœess calcaires et lœss calcaires lités (unités 2a à 2d) ;

- sol brun lessivé plus ou moins tronqué (1) ;

- colluvions récentes $(0)$.

L'étude de la stratigraphie de la formation de VilliersAdam permet donc de mettre en évidence trois grandes séquences de type glaciaire-interglaciaire partiellement emboîtées (fig. 11). La cyclicité déduite de l'approche pédosédimentaire est par ailleurs nettement soulignée par les courbes de variations de paramètres comme le pourcentage d'argile, de fer et de carbone organique, par exemple (fig. 9, 10, 13 et 14). Dans le secteur de Villiers-Adam, la conservation de cette épaisse formation pléistocène, dans un contexte où la sédimentation lœessique typique est peu développée, est vraisemblablement en liaison avec la présence d'un substratum tertiaire sableux très sensible aux processus d'érosionsédimentation en milieu froid (périglaciaire) :

- accentuation des processus d'érosion par ruissellement concentré lors des phases de dégradation du permafrost (processus thermokarstiques: Murton, French, 1992, 1993 ; Van Vliet-Lanoë, 1996) avec création de pièges sédimentaires sous la forme de vallons parallèles à la pente ;

- source importante de matériaux locaux très sensibles à la déflation éolienne.

D'une manière générale, l'étude pédostratigraphique de la formation de Villiers-Adam débouche sur la description d'une succession de trois grands cycles de type glaciaire-interglaciaire, dont les traits majeurs sont corrélables avec ceux de la stratigraphie du Pléistocène moyen récent et du Pléistocène supérieur de l'Europe du Nord-Ouest (Antoine et al., 1998). 

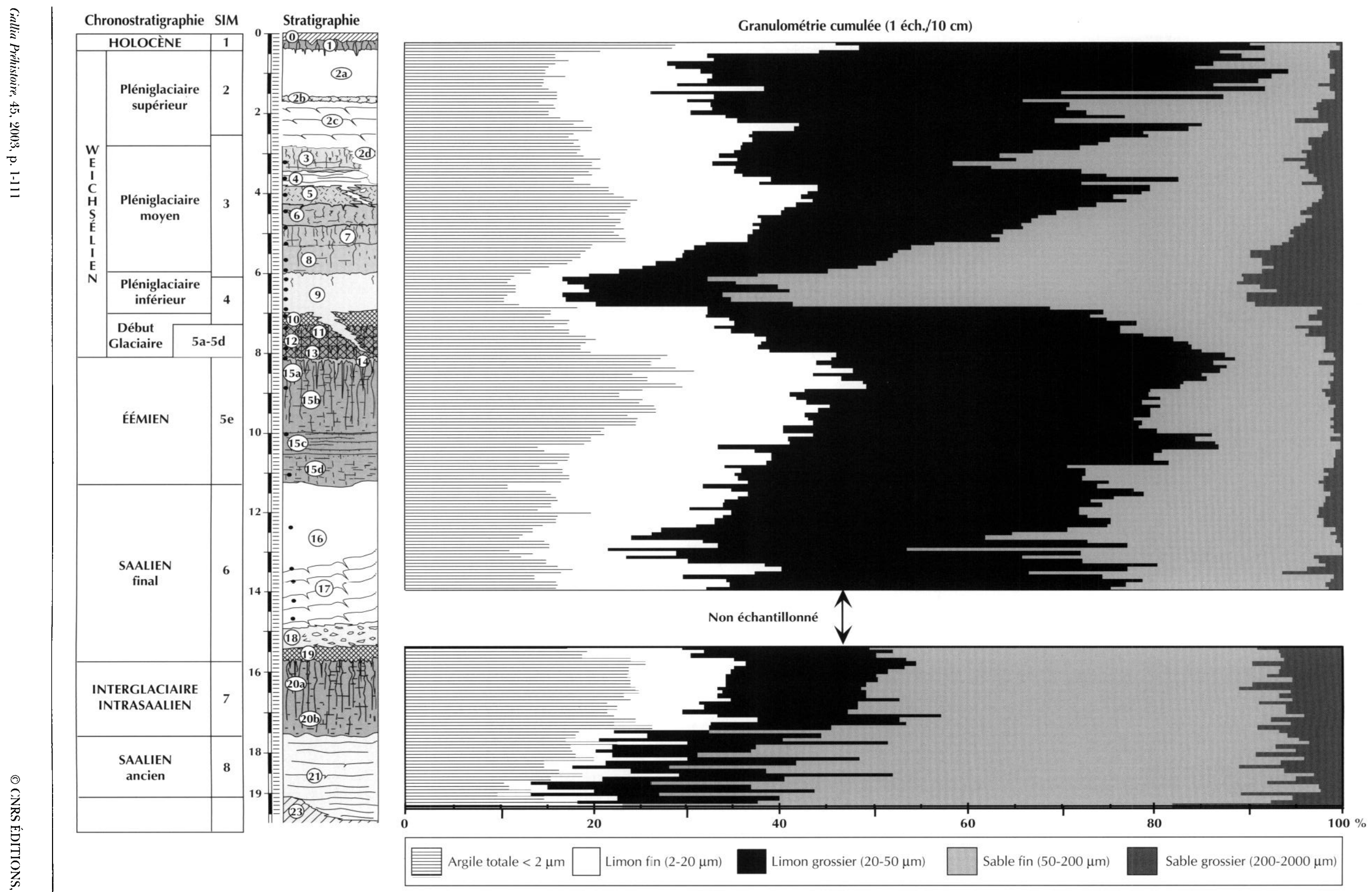

Fig. 13 - Villiers-Adam, Le Chamesson : séquence synthétique, données granulométriques cumulées, un échantillon tous les $10 \mathrm{~cm}$ (description des unités, voir tabl. I), dessin P. Antoine. 


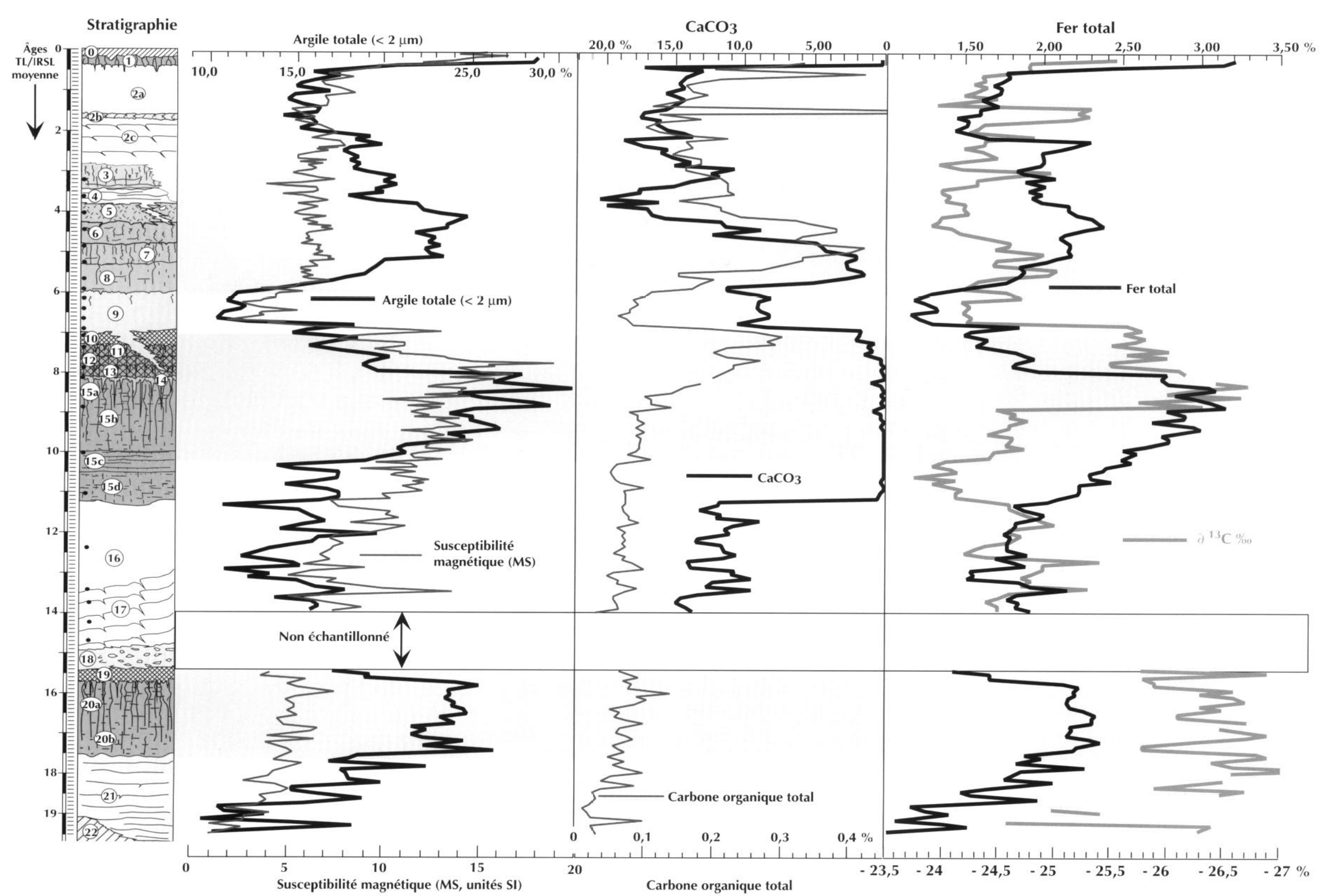

Fig. 14 - Villiers-Adam, Le (hamesson : séquence synthétique, évolution verticale des valeurs de susceptibilité magnétique, des pourcentages d'argile (< 2 pum), de carbone organique tolal, de fer total, de $\mathrm{CaCO}_{3}$ et du $\delta^{\prime 3}$ (' (description des unités, voir tabl. I), dessin I. Antoine. 
Sur cette base, et indépendamment des données géochronologiques, il est possible de proposer les interprétations chronostratigraphiques globales suivantes.

Séquence 1 : Glaciaire saalien (stade isotopique 8 ?) Interglaciaire intrasaalien (SI 7) ;

Séquence 2: Glaciaire saalien supérieur (SI 6) Interglaciaire éémien (SI 5e) ;

Séquence 3: Glaciaire weichsélien (SI $5 \mathrm{~d}$ à 2) Interglaciaire holocène (SI 1).

\section{DONNÉES BIOCLIMATIQUES ET GÉOCHIMIQUES}

\section{ANALYSE POLLINIQUE}

\section{INTRODUCTION}

Les enregistrements polliniques sont rarement conservés dans les dépôts loessiques et les paléosols. Aussi l'ensemble des unités stratigraphiques du Chamesson et du Petit Saule a-t-il fait l'objet, dans un premier temps, de prélèvements palynologiques tests. Ils étaient destinés à vérifier la concentration et la conservation du contenu sporo-pollinique de ces différents niveaux et à retenir les séries les plus pertinentes pour envisager une reconstitution des végétations et des climats qui les ont conditionnées et de caractériser les milieux des occupations humaines de Villiers-Adam.

\section{Prélèvements}

Les formations saaliennes (couches 21, 20, 17 et 15d) prélevées au Chamesson se sont révélées impropres à une étude palynologique (fig. 8, tabl. II). Par ailleurs, le $\mathrm{B}_{\mathrm{t}}$ de sol éémien (couches $15 \mathrm{~b}$ et 15a) échantillonné au Chamesson et au Petit Saule est stérile en grains de pollen (fig. 8, tabl. II et III).

Le complexe de sols du Début Glaciaire weichsélien (couches 13, 12, 11 et 10) testé au Chamesson et au Petit Saule n'a pas donné de résultats exploitables (fig. 8, tabl. II et III).

En revanche, les tests réalisés sur la série du Pléniglaciaire inférieur (couche 9) et moyen (couches 8, 7, 6,

Tabl. II - Résultats des tests palynologiques réalisés dans les différentes unités stratigraphiques du site de Villiers-Adam, Le Chamesson.

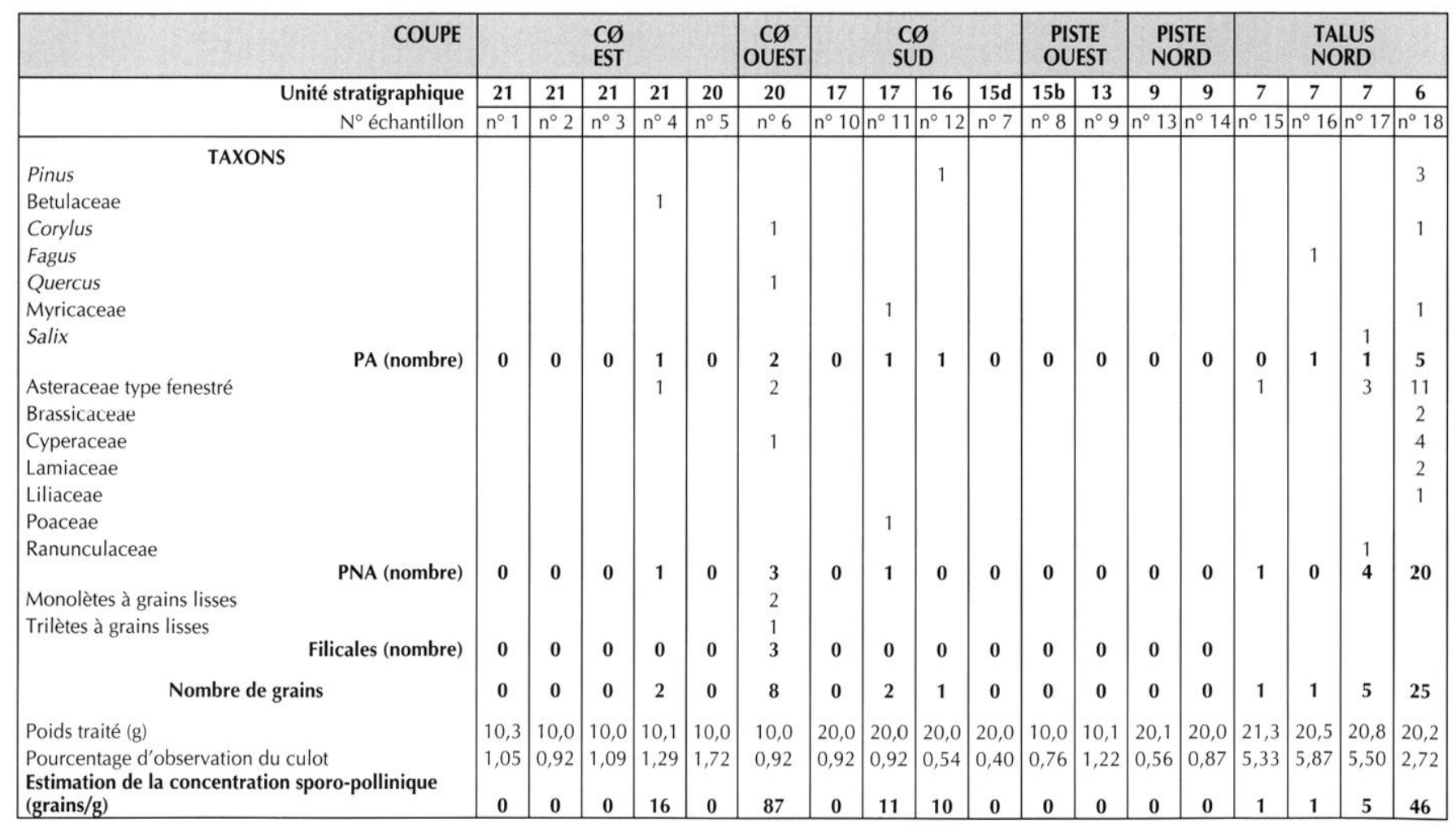


Tabl. III - Résultats des tests palynologiques réalisés dans les différentes unités stratigraphiques du site de Villiers-Adam, Le Petit Saule.

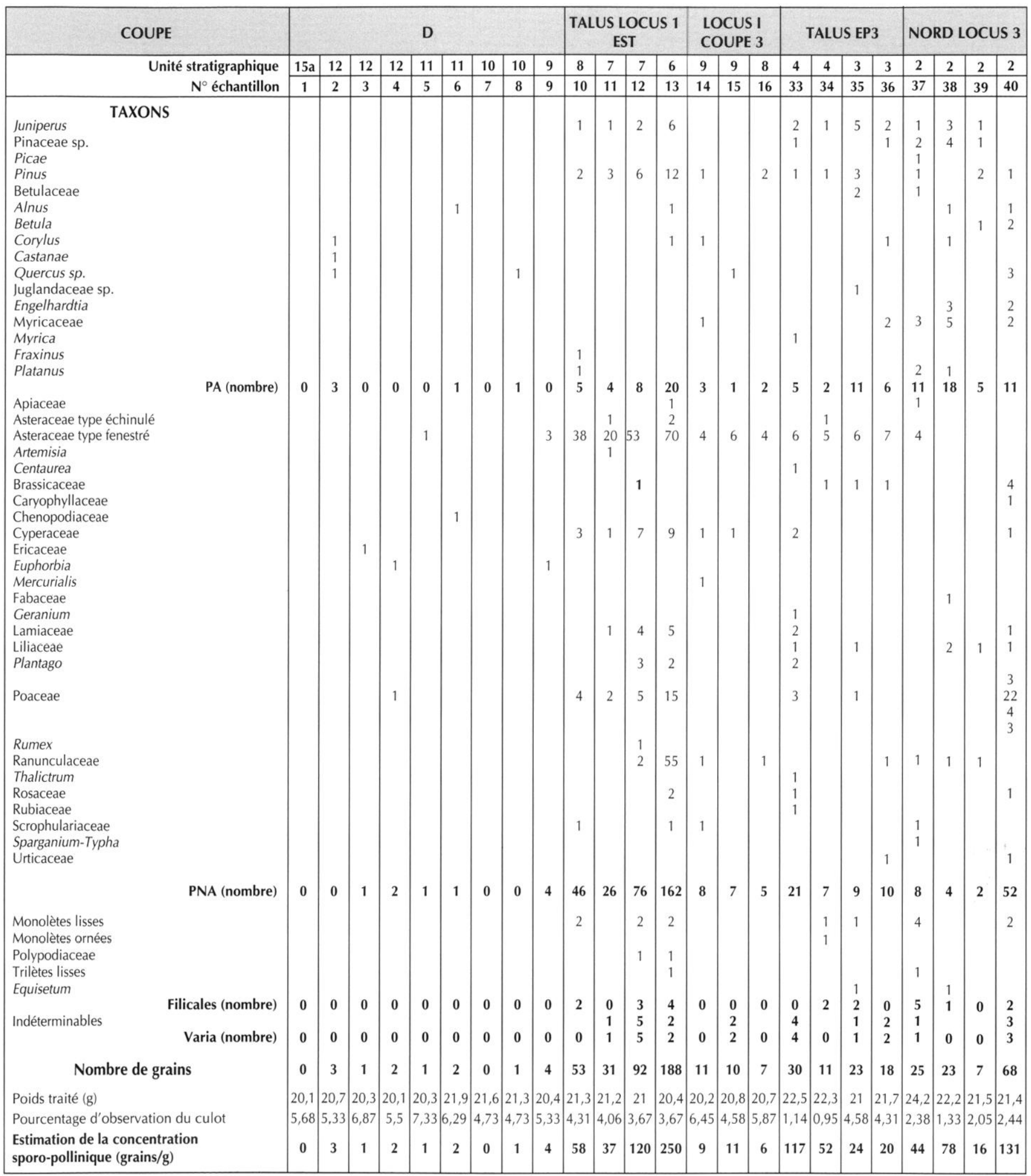


Fig. 15 - Villiers-Adam, Le Petit Saule : séquences du Début Glaciaire et du Pléniglaciaire moyen du Weichsélien, coupes Talus locus 1-EP3, Talus lorus 1-est et $D$, avec position des prélèvements malacologiques et palynologiques (dessin J.J. Bahain et P. Raymond).

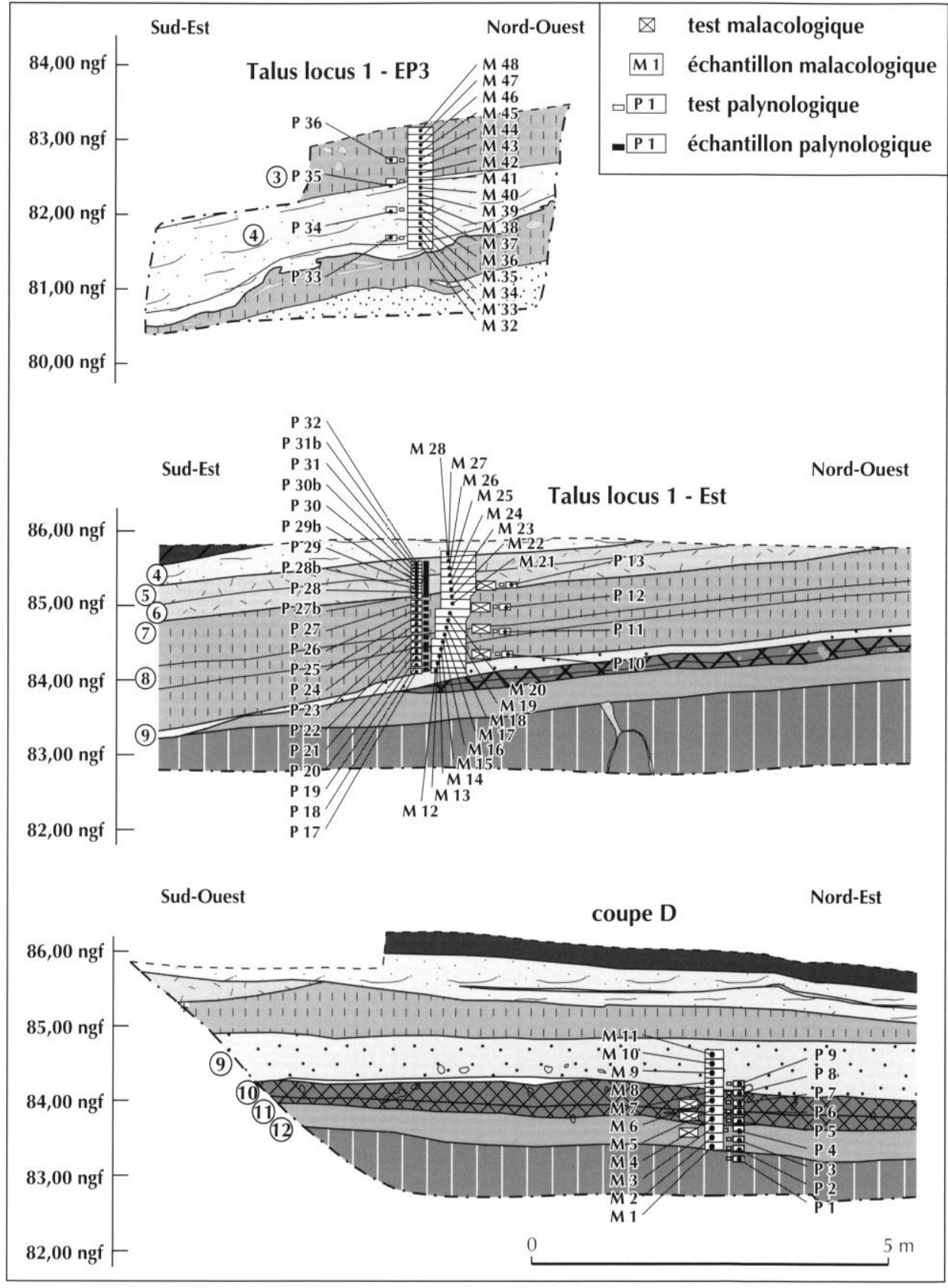

4 échantillons au Petit Saule, complétés par une série de 21 échantillons couvrant les couches stratigraphiques 9 à 5 (fig. 8 et 15 ).

\section{MÉTHOdologiE}

Les échantillons ont subi un traitement chimique classique (Faegri, Iversen, 1989) suivi d'une flottation en liqueur dense. La lecture des lames au microscope a été réalisée à l'objectif 16 et toutes les déterminations ont été effectuées à l'objectif 100. 
Le comptage a été guidé pour l'observation de seuils de fiabilité statistique : comptage d'au moins 300 sporomorphes (pollens et spores) avec au moins 100 grains en plus du taxon dominant, observation d'un minimum de 20 taxons polliniques.

Les résultats sont présentés sous différentes formes graphiques.

Dans les diagrammes polliniques détaillés, les pourcentages de tous les taxons polliniques sont calculés par rapport à la somme de base PA (pollen arboréen) + PNA (pollen non arboréen). Les pourcentages des différentes spores sont calculés par rapport au nombre total (PA + PNA + filicales + indéterminables).

En raison de la forte représentation des Asteraceac type fenestré au Petit Saule, un second diagramme détaillé est présenté dans lequel ce taxon a été exclu de la somme de base et du nombre total.

Les diagrammes polliniques synthétiques sont basés sur le regroupement des taxons d'écologie similaire, facilitant la visualisation des résultats, et constituent une première interprétation des diagrammes détaillés.

Les taxons identifiés lors de l'analyse ont permis d'établir quatre grands groupes dont les pourcentages sont basés sur la somme de base moins les taxons remaniés (Juglandaceae sp., Engelhardtia, Myricaceae sp.). Dans le second diagramme pollinique synthétique, les Asteraceae type fenestré ont été exclues de la somme de base. De gauche à droite sont distingués les groupes suivants.

Groupe 1. Arbres et arbustes tempérés: Alnus (aulne), Betula (bouleau), Corylus (noisetier), Quercus sp. (chêne caducifolié), Fraxinus (frêne), Platanus (platane), Populus (peuplier), Salix (saule). Ce groupe ne tient pas compte des besoins édaphiques des différents taxons.

Groupe 2. Arbres à pollens à ballonnets : Pinaceae sp., Pinus (pin). Pinus est un très bon producteur et disperseur pollinique. Le distinguer du groupe des feuillus permet de visualiser une possible surreprésentation.

Groupe 3. Herbacées ubiquistes ou sans signification : Apiaceae, Borraginaceae, Brassicaceae, Campanulaceae, Cyperaceae, Ericaceae, Euphorbia (euphorbe), Mercurialis (mercuriale), Fabaceae, Geranium (géranium), Lamiaceae, Liliaceae, Nymphaeaceae, Plantago (plantain), Polygonum (renouée), Rumex (petite oseille), Ranunculaceae, Thalictrum, Rosaceae, Filipendula (filipendule), Sanguisorba minor (pimprenelle), Sanguisorba officinalis (sanguisorbe officinale), Rubiaceae, Scrophulariaceae,
Sparganium-Typha (rubanier), Typha latifolia (massette à larges feuilles), Urticaceae, Valerianaceae. Il faut souligner que plusieurs de ces taxons possèdent des caractéristiques particulières et sont notamment soit des aquatiques (Nymphaeaceae, Sparganium-Typha et Typha latifolia), soit des plantes de milieux humides (Cyperaceae, Filipendula), mais leurs faibles taux ne permettaient pas de les distinguer dans un autre groupe.

Groupe 4. Herbacées et gymnospermes xérophiles et héliophiles: Asteraceae à grains échinulés, Asteraceae à grains fenestrés, Artemisia (armoise), Centaurea (centaurée), Caryophyllaceae, Chenopodiaceae, Helianthemum (hélianthème) et Juniperus (genévrier), auxquelles sont jointes les Poaceac (graminées).

Le diagramme partiel des concentrations (calculées selon la méthode volumétrique: Cour, 1974) établi pour la coupe du Petit Saule a été utilisé comme élément de comparaison avec les deux autres types de diagrammes. Les courbes polliniques des fréquences absolues étant indépendantes les unes des autres, chaque taxon peut être considéré sans l'influence relative des autres taxons. Ainsi, les variations des fréquences absolues permettent de contrôler si les variations des fréquences relatives (ou pourcentages) sont réelles ou dues à des aléas statistiques. De plus, il a été montré (Van Campo, 1984; Follieri et al., 1988) que la concentration sporo-pollinique pouvait refléter la densité du couvert végétal.

\section{RÉSULTATS}

\section{Analyse}

La flore déterminée comprend 50 taxons polliniques correspondant à 36 familles de gymnospermes et d'angiospermes. Les déterminations ont été faites à des rangs systématiques différents (tabl. IV) dont 24 au niveau de la famille, 22 au niveau du genre et 3 au niveau de l'espèce. Cinq taxons ont été distingués dans les ptéridophytes; ils correspondent à 4 types polliniques et à 1 famille. La majorité des spores trilètes ornées semble s'apparenter au taxon Cryptogramma crispa. Cette attribution se base sur des comparaisons avec des clichés photographiques (Moore et al., 1991; Reille, 1992, 1995, 1998), mais par prudence il n'a pas été dissocié des spores trilètes ornées dans les diagrammes détaillés. 
Tabl. IV - Liste botanique de Villiers-Adam.

\begin{tabular}{|c|c|c|}
\hline PTERIDOPHYTAE & Polypodiaceae & $s p$. \\
\hline GYMNOSPERMAE & $\begin{array}{l}\text { Betulaceae } \\
\text { Borraginaceae } \\
\text { Brassicaceae } \\
\text { Campanulaceae } \\
\text { Caryophyllaceae } \\
\text { Chenopodiacaee } \\
\text { Cistaceae } \\
\text { Cyperaceae } \\
\text { Ericaceae } \\
\text { Euphorbiaceae } \\
\\
\text { Fabaceae } \\
\text { Fagaceae } \\
\text { Geraniaceae } \\
\text { Juglandaceae } \\
\text { Lamiaceae } \\
\text { Liliaceaeae } \\
\text { Myricaceae } \\
\text { Nymphaeaceae } \\
\text { Oleaceae } \\
\text { Plantaginaceae } \\
\text { Platanaceae } \\
\text { Poaceae } \\
\text { Polygonaceae } \\
\text { Ranunculaceae } \\
\text { Rosaceae } \\
\text { Rubiaceae } \\
\text { Salicaceae } \\
\text { Scrophulariaceae } \\
\text { Sparganiaceae } \\
\text { Typhaceae } \\
\text { Urticaceae } \\
\text { Valerianaceae }\end{array}$ & $\begin{array}{l}\text { Juniperus } \\
\text { sp. } \\
\text { Pinus } \\
\text { sp. } \\
\text { Liguliflorae = grains fenestrés } \\
\text { Tubuliflorae = grains } \\
\text { échinulés } \\
\text { Artemisia } \\
\text { Centaurea } \\
\text { Alnus } \\
\text { Betula } \\
\text { Corylus } \\
\text { sp. } \\
\text { sp. } \\
\text { sp. } \\
\text { sp. } \\
\text { sp. } \\
\text { Helianthemum } \\
\text { sp. } \\
\text { sp. } \\
\text { Euphorbia } \\
\text { Mercurialis } \\
\text { sp. } \\
\text { Quercus sp. } \\
\text { Geranium } \\
\text { sp. } \\
\text { Engelhardtia } \\
\text { sp. } \\
\text { sp. } \\
\text { sp. } \\
\text { sp. } \\
\text { Fraxinus } \\
\text { Plantago } \\
\text { Platanus } \\
\text { sp. } \\
\text { Polygonum } \\
\text { Rumex } \\
\text { sp. } \\
\text { Thalictrum } \\
\text { sp. } \\
\text { Filipendula } \\
\text { Sanguisorba minor } \\
\text { Sanguisorba officinalis } \\
\text { sp. } \\
\text { Populus } \\
\text { Salix } \\
\text { sp. } \\
\text { Sparganium-Typha } \\
\text { Typha latifolia } \\
\text { sp. } \\
\text { sp. } \\
\end{array}$ \\
\hline
\end{tabular}

\section{Conservation et concentration}

Les lames microscopiques des échantillons provenant des deux sites présentent un fond particulièrement dense en microcristaux et microdébris opaques, qui doivent correspondre à des minéraux opaques et peu chargés en matière organique à éléments figurés correspondant à des microdébris végétaux.

La conservation pollinique de tous les échantillons est très médiocre. L'état de conservation très altéré des grains de Pinus explique qu'un certain nombre de grains de pollen bi-ailés ait été regroupé en Pinaceae sp.

Les échantillons étudiés possèdent une estimation de la concentration sporo-pollinique très basse, inférieure à 1000 grains par gramme de sédiment. La moyenne des fréquences absolues pour les spectres polliniques se situe autour de 210 grains par gramme de sédiment.

Les spectres polliniques se caractérisent par une surreprésentation des Asteraceae à grains fenestrés. Ce constat, conjugué à la mauvaise concentration, indique qu'une altération a affecté le contenu sporo-pollinique des sédiments (Couteaux, 1977 ; Morzadec-Kerfourn, 1977 ; Reille, 1978; Hall, 1981). Afin d'atténuer cette source d'erreur due à l'altération et à la conservation différentielle, les spectres s'approchent le plus possible des conditions des seuils de fiabilité statistique déjà évoqués.

Une autre observation peut être reliée à la mauvaise concentration. Le long de la séquence, du pollen frais (non comptabilisé dans les spectres polliniques) a été rencontré, signalant des pollutions actuelles, phénomène également mis en évidence par l'étude des malacofaunes (voir p. 37). De plus, les notations de taxons antéquaternaires (Pokrovskaia, 1958 ; Gruas-Cavagnetto, 1968 ; Van der Hammen et al., 1971; Chateauneuf, 1974; Gruas-Cavagnetto, 1977), régulièrement enregistrées le long de la coupe, témoignent de reprises de terrains tertiaires. Ces deux faits illustrent clairement que la moindre contamination par du pollen de tout âge (actuel ou ancien) dans des sédiments très pauvres en pollen "prend des proportions rédhibitoires" (Pons, 1984).

Les faibles fréquences absolues des sédiments, l'altération du contenu sporo-pollinique, la conservation différentielle des palynomorphes indiquent que les données apportées par les spectres polliniques sur la flore, la végétation et le climat seront amputées d'une partie des informations.

\section{Interprétation}

Le spectre pollinique obtenu sur le niveau stratigraphique 6 du Chamesson (fig. 16) présente les mêmes caractéristiques que celles des spectres issus du même 


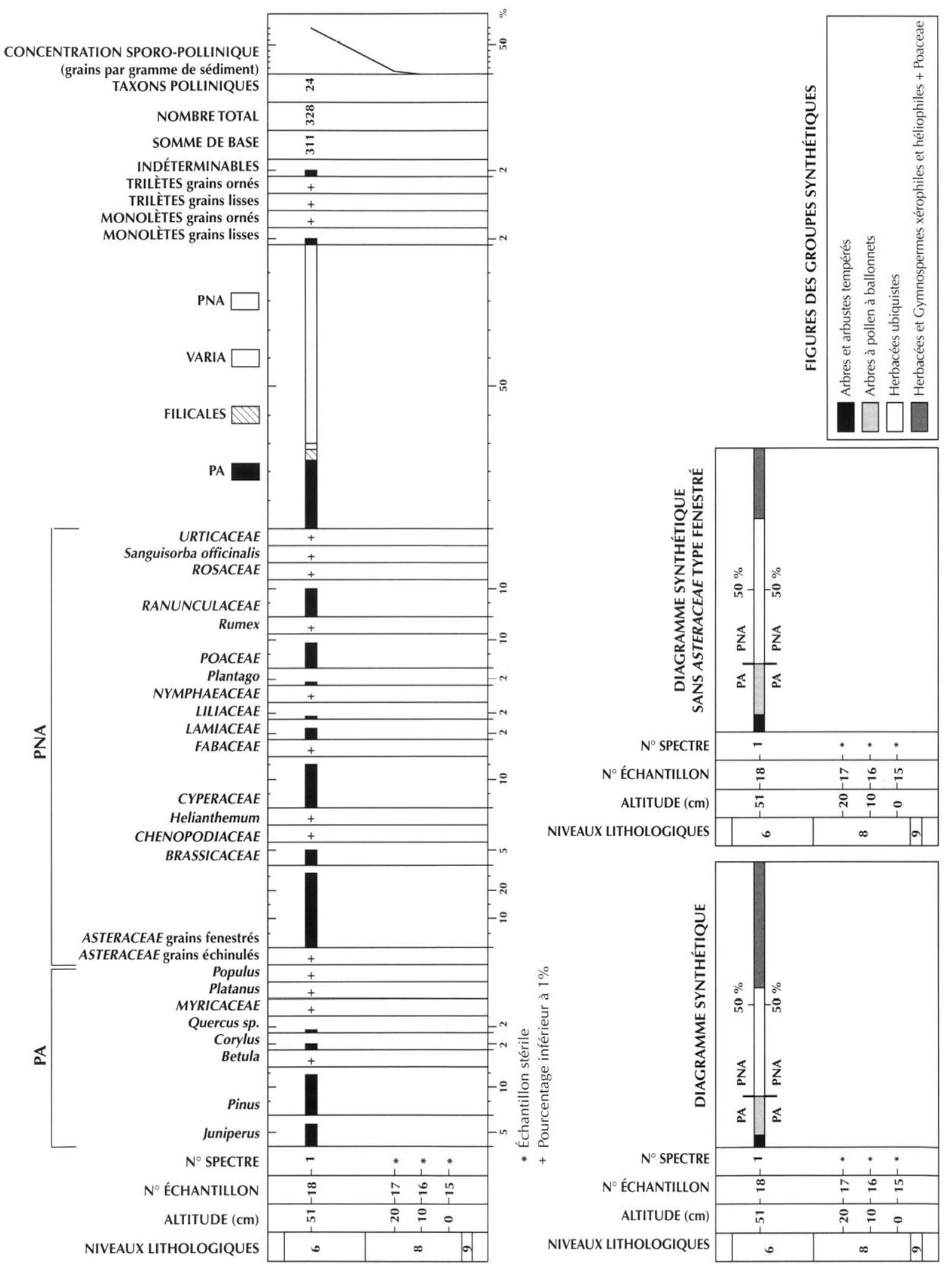


niveau au Petit Saule. L'interprétation des données polliniques se base donc sur les résultats de la coupe Talus locus I-est du Petit Saule (fig. 17 à 20).

Le diagramme pollinique (fig. 17) présente une grande homogénéité, qui se caractérise par :

- la rareté du pollen arboréen, principalement constitué par Juniperus et Pinus;

- la prépondérance des herbacées, dominées largement par les Asteraceae de type fenestré et dans une moindre mesure par les Poaceae et Cyperaceae ;

- la présence faible mais continue d'Artemisia, des Caryophyllaceae, des Chenopodiaceae, Helianthemum et Thalictrum;

- la constance des taux faibles des filicales ;

- une augmentation de la base au sommet de la concentration totale.

La comparaison des différentes représentations graphiques (fig. 16 à 20) a facilité le découpage de la séquence en deux zones polliniques d'après les fluctuations d'au moins deux courbes de taxons importants du point de vue écologique ou dynamique (Birks, Birks, 1980 ; Beaulieu et al., 1985).

\section{Zone pollinique a}

Cette zone est représentée par 9 spectres polliniques et comporte à la base 4 échantillons stériles (fig. 17 et 18). La concentration sporo-pollinique est très faible, oscillant entre 50 et 200 grains par gramme de sédiment (fig. 20). Le nombre de taxons polliniques se situe entre 17 et 21 (fig. 17 et 18 ).

Les pourcentages très bas du PA sont relativement constants et ne dépassent pas les $20 \%$ du nombre total (entre $7 \%$ et $19 \%$ ). Les taxons arboréens sont représentés par Juniperus (4-17\%) et Pinus (2-4\%). Des occurrences irrégulières de Alnus, Betula, Populus et Salix sont enregistrées. Ce dernier taxon n'est plus signalé au-delà du spectre 6 (échantillon $n^{\circ} 24$ ). Les notations sporadiques de Juglandaceae, Engelhardtia, Myricaceae sont à mettre en relation avec une reprise de sédiments éocènes, tandis que celles de Quercus sp., Plalanus et Fraxinus semblent plus témoigner d'une perturbation subactuelle ou d'un apport lointain extrarégional que d'une reprise des sédiments antérieurs déposés lors d'une période tempérée, les tests palynologiques ayant montré leur stérilité.

Parmi les herbacées (PNA entre $72 \%$ et $93 \%$ ), les Asteraceae type fenestré sont dominantes (42-68\%). Les
Poaceae (6-18\%), Cyperaceae (1-12\%), Ranunculaceae (1-4\%), Plantago (1-5\%) et Lamiaceae (1-2\%) sont également bien représentées. Des occurrences régulières d'Asteraceae type échinulé, de Brassicaceae, Liliaceae, Nymphaeaceae, Rosaceae sont enregistrées. Artemisia, Caryophyllaceae, Helianthemum, Thalictrum se signalent par des notations discontinues.

La courbe des fréquences des filicales est régulière et principalement due aux valeurs des spores de monolètes.

Les diagrammes synthétiques (fig. 19) montrent clairement la dominance du groupe des xérophileshéliophiles et Poaceae sur les autres herbacées. Les deux autres groupes sont pratiquement inexistants.

\section{Zone pollinique $b$}

Cette zone correspond aux 12 échantillons supérieurs (fig. 17 et 18). Le spectre pollinique obtenu au Chamesson (fig. 16) peut lui être corrélé, tant par ses caractéristiques polliniques que par son appartenance à la couche stratigraphique 6 .

L'allure générale de la concentration sporopollinique montre une augmentation générale (aussi bien du pollen arboréen que du pollen herbacé) se situant entre 100 et 530 grains par gramme de sédiment (fig. 20). Le nombre de taxons n'est pas très élevé, il se situe entre 14 et 23.

Le PNA est toujours important $77-93 \%$ du nombre total). Il est dominé par les Asteraceae type fenestré, dont les fréquences relatives ont légèrement diminué (40-75\%). Les courbes des pourcentages des Cyperaceae et Poaceae s'inversent par rapport à la zone a (les premières ayant une meilleure représentation que les secondes). Les taux des Asteraceae type échinulé, Brassicaceae, Lamiaceae, Plantago, Ranunculaceae, Rosaceae sont en légère augmentation. Les Caryophyllaceae, Fabaceae, Liliaceae, Nymphaeaceae, Thalictrum, Rubiaceae, Urticaceae montrent des notations régulières. Des occurrences discontinues de Centaurea, Campanulaceae, Ericaceae, Polygonum, Rumex, Filipendula ne sont enregistrées que dans cette zone pollinique b. Le diagramme réalisé en excluant les Asteraceae type fenestré (fig. 18) et le diagramme des concentrations (fig. 20) montrent clairement que cette zone correspond à une diversification et une augmentation des fréquences des herbacées.

Le taux de PA est faible (6-16\% du nombre total). Les fréquences de Pinus sont en légère hausse (1-7\%), tandis 


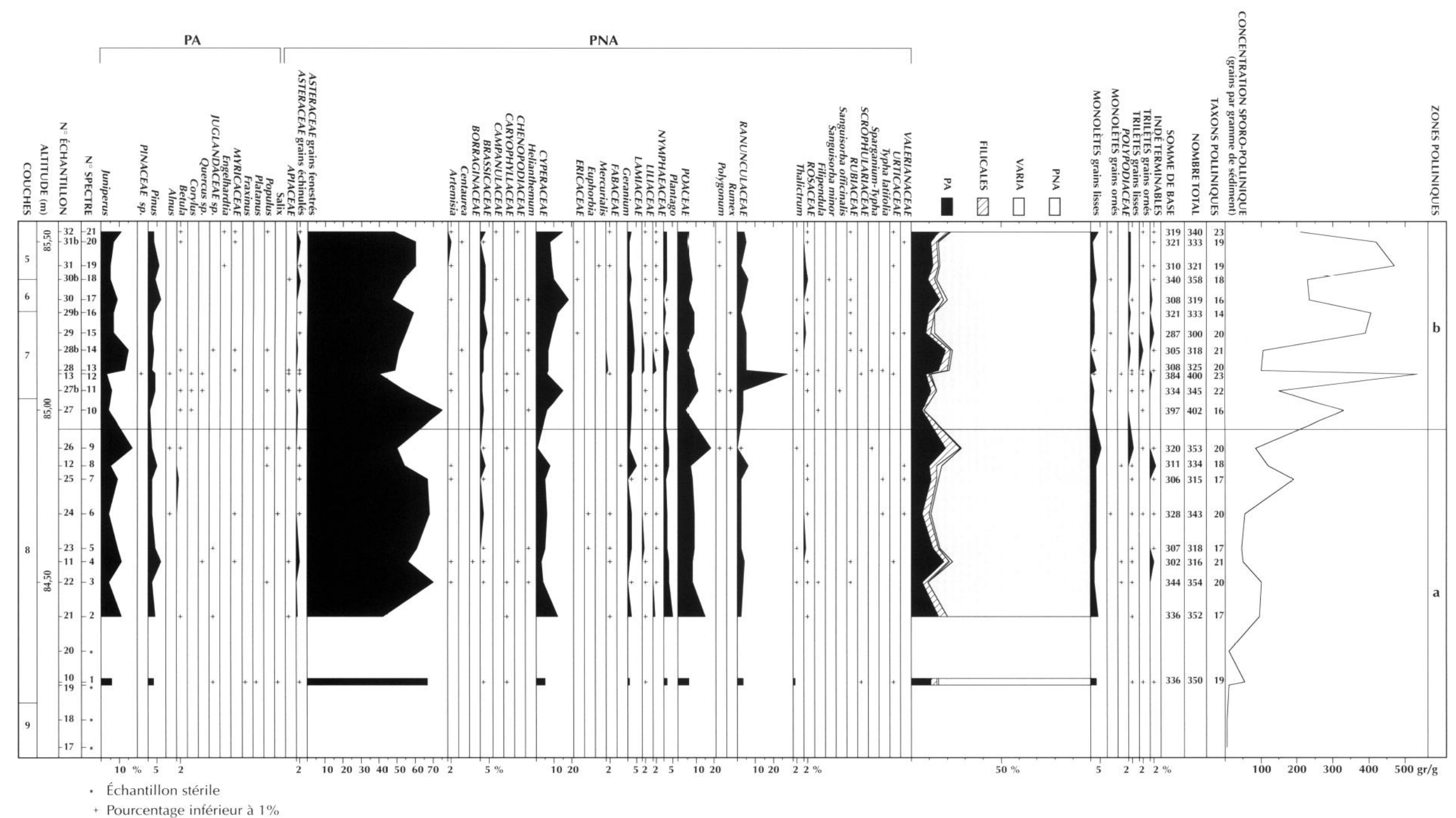

Fig. 17 - Villiers-Adam, Le Petit Saule : diagramme détaillé (dessin A. Gauthier).

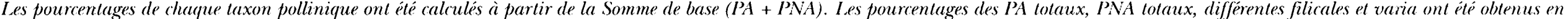

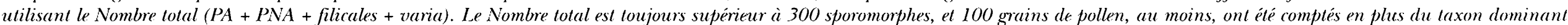




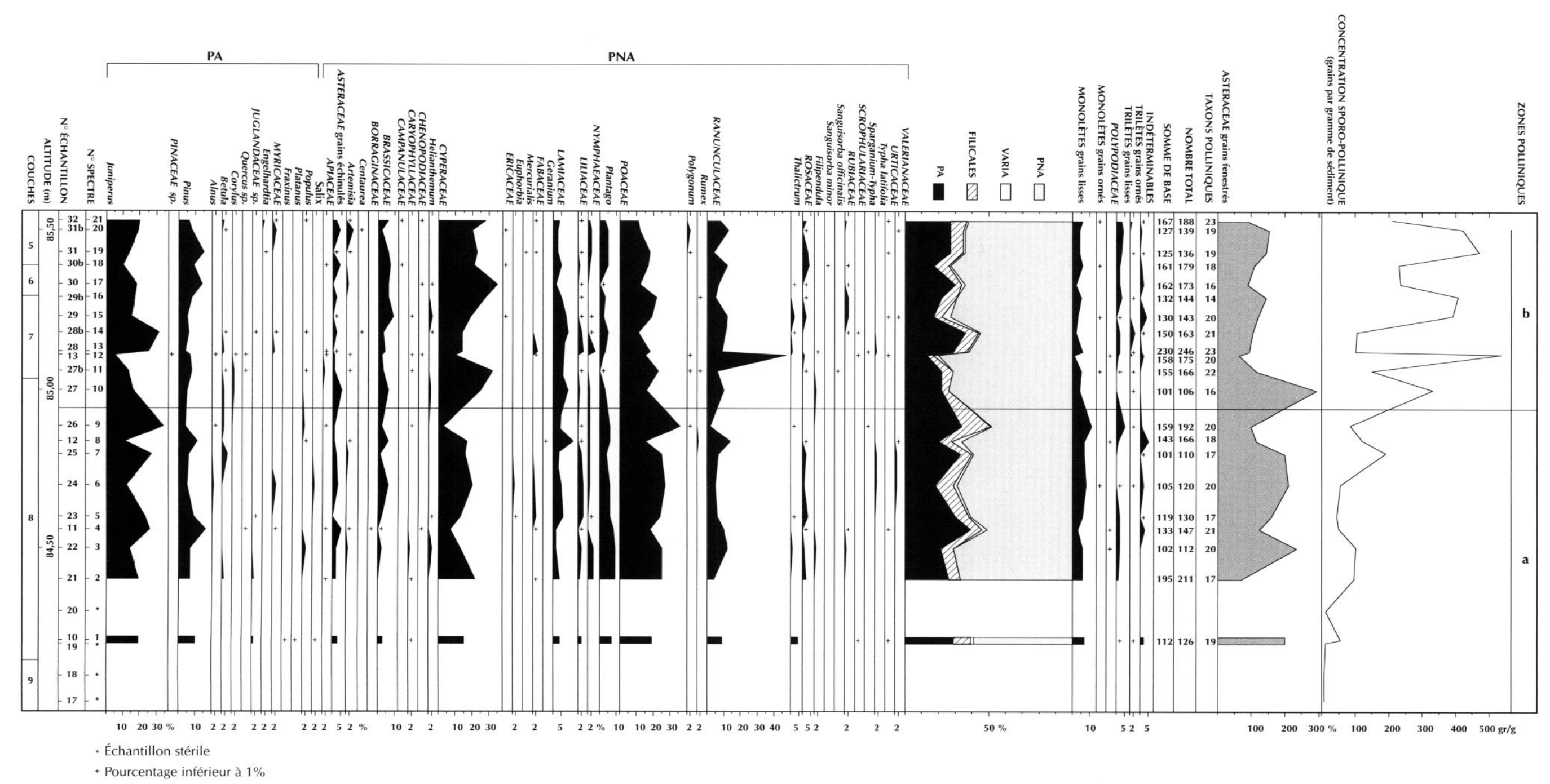

Fig. 18 - Villiers-Adam, Le Petit Saule : diagramme détaillé, Asteraceae type fenestré exclues du nombre total (dessin A. Gauthier).

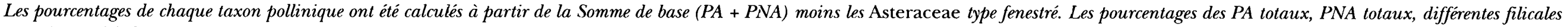

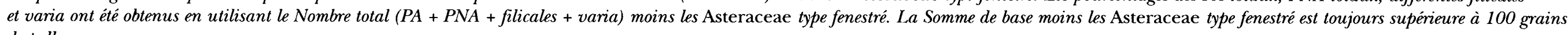
de pollen. 
DIAGRAMME SYNTHÉTIQUE

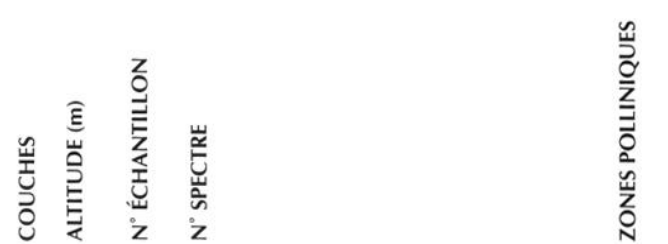

DIAGRAMME SYNTHÉTIQUE

Asteraceae type fenestré exclues

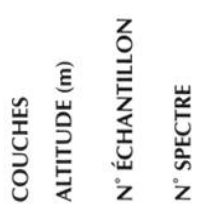

岂

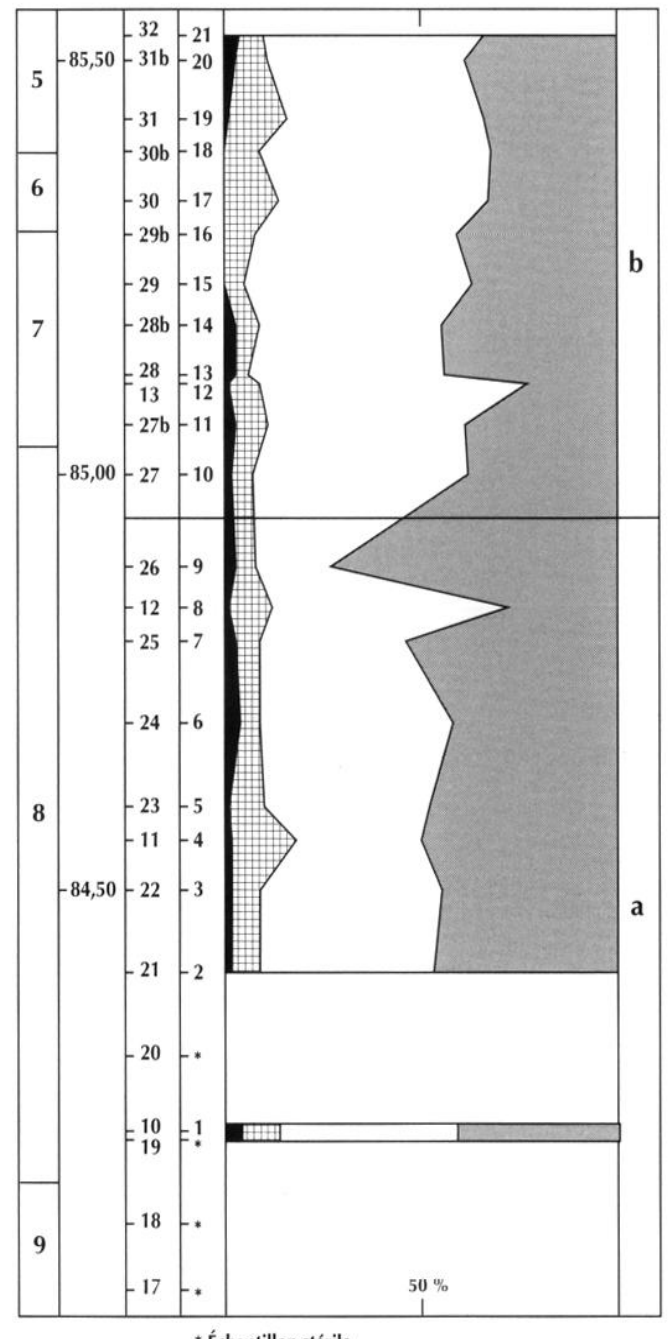

•Échantillon stérile

FIGURES DES GROUPES SYNTHÉTIQUES

Arbres et arbustes tempérés Herbacées ubiquistes et aquatiques

Arbres à pollen à ballonnets

Herbacées et Gymnospermes xérophiles et héliophiles + Poaceae

Fig. 19 - Villiers-Adam, Le Petit Saule : diagrammes synthétiques (dessin A. Gauthier). 


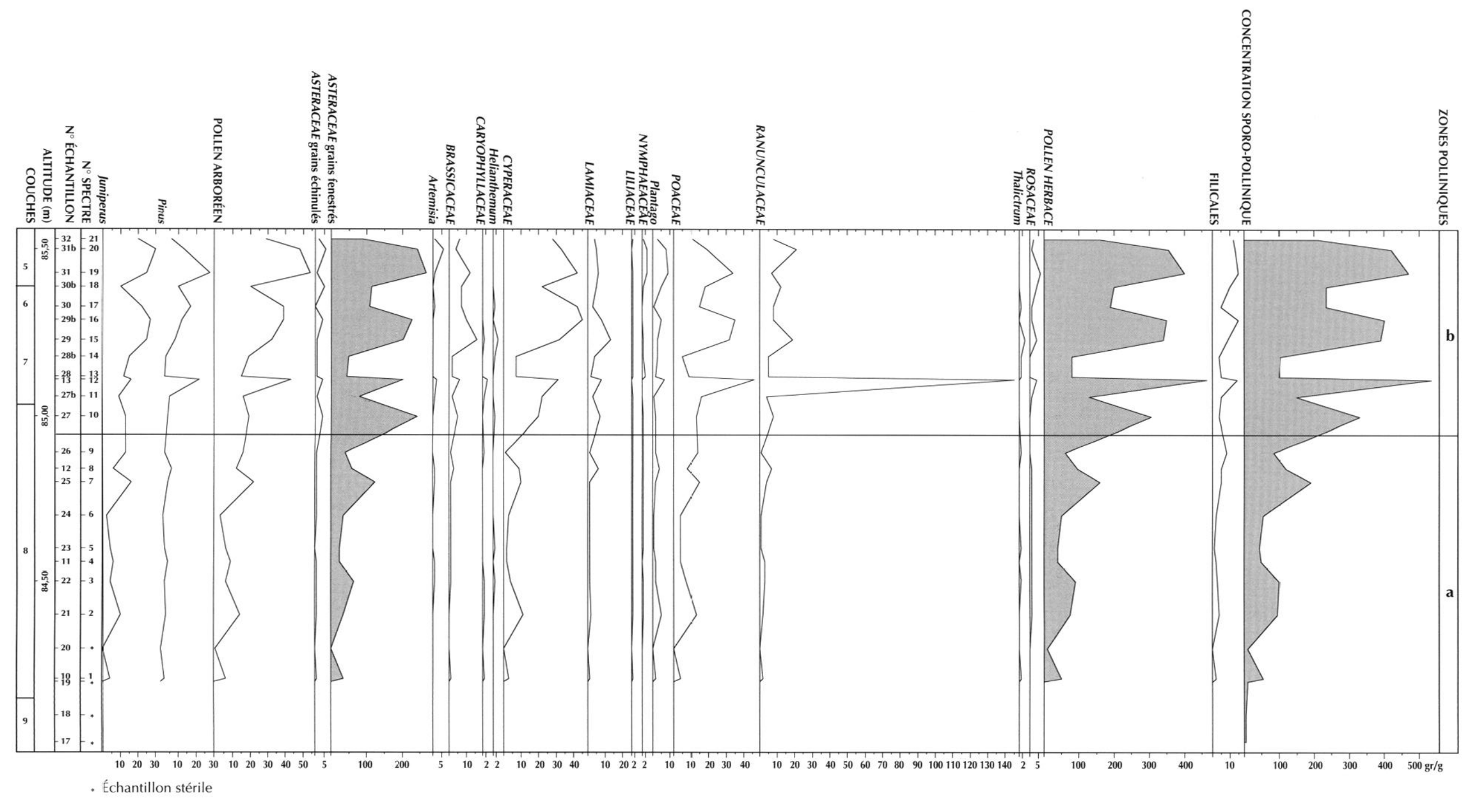

Fig. 20 - Villiers-Adam, Le Petit Saule : diagramme partiel des concentrations (dessin A. Gauthier). 
que celles de Juniperus sont stables. Des notations continues de Betula et Populus sont enregistrées. Des remaniements (tertiaire et actuel) sont toujours signalés par des grains isolés de Juglandaceae, Engelhardtia, Myricaceae et Quercus.

Les taux des filicales sont constants par rapport à la zone pollinique a. Toutefois, les fréquences relatives des spores monolètes ont diminué, alors que celles des trilètes sont en augmentation. Parmi ces dernières, il faut remarquer les notations régulières des trilètes grains ornés, dont la majorité semblent s'apparenter à Cryptogramma crispa.

Les diagrammes synthétiques (fig. 16 et 19) soulignent la meilleure représentation des herbacées ubiquistes par rapport au groupe des xérophiles-héliophiles et des Poaceae. Les deux autres groupes restent à leurs taux précédents.

\section{RECONSTITUTION DE LA VÉGÉTATION}

Du point de vue biogéographique, les sites de VilliersAdam se trouvent dans le domaine atlantique de la région euro-sibérienne (Ozenda, 1964).

L'étude de la représentation pollinique actuelle de chaque taxon particulier et des différentes formations végétales (Heim, 1970 ; Beaulieu, 1977 ; Huntley, Birks, 1983 ; Barthélemy, 1985 ; Reille, 1990a ; Jolly, 1994 ; Ruffaldi, 1994) permet d'interpréter les zones polliniques en termes de végétation.

Les valeurs du PNA très élevées dans les deux zones polliniques reflètent une formation ouverte. Le couvert herbacé important est composé principalement de taxons héliophiles et xérophiles: Asteraceae type fenestré dominant largement, accompagnées de Poaceae, Cyperaceae, Plantago, Artemisia, Caryophyllaceae, Chenopodiaceae, Asteraceae type échinulé, Helianthemum, Thalictrum auxquels s'ajoutent d'autres herbacées à caractère ubiquiste telles que Brassicaceae, Lamiaceae, Liliaceae, Ranunculaceae et Juniperus.

La composition de cette formation appelle plusieurs remarques.

Suite au dépôt de la couche 9 , stérile en pollen, la meilleure concentration sporo-pollinique à partir de la base de la couche 8 permet d'obtenir des spectres polliniques (fig. 17). Durant la zone pollinique a (couvrant pratiquement la couche 8 ), la concentration augmente mais reste à des valeurs faibles, soulignant la pauvreté floristique de cette zone. L'augmentation des concentrations totales est conjointe à la hausse des fréquences relatives et absolues de Juniperus (fig. 17 et 20), ce qui témoigne d'un meilleur développement de ce taxon. Un nouveau changement se manifeste au passage des couches 8-7 par une nouvelle augmentation de la concentration (fig. 20). Il permet d'indiquer un couvert végétal plus dense et une végétation floristiquement plus riche que précédemment.

La forte dominance des Asteraceae type fenestré est due, en grande partie, à une conservation différentielle, les données sur les végétations actuelles formées de nombreuses composées ne permettant pas d'obtenir des pourcentages très élevés de ce taxon (Bottema, 1975). Toutefois, la présence de pollinies de ce taxon dans plusieurs spectres montrent leur présence locale. Les pourcentages des autres taxons sont masqués par l'abondance de ces Asteraceae.

Les spectres polliniques (fig. 17) se caractérisent par la courbe assez sporadique d'Artemisia, taxon steppique. Cette rareté d'Artemisia dans des sédiments pléniglaciaires, à la différence du Tardiglaciaire où cette essence connaît un essor fulgurant, a déjà été soulignée par de nombreux auteurs.

La majorité des grains de Plantago déterminés correspond au type operculé. Les grains de ce type peuvent correspondre à des espèces steppiques, comme $P$. lagopus, P. argentea (Fournier, 1977; Reille, 1992), ou à des espèces indicatrices d'anthropisation, telles que $P$. lanceolata, P. major. La présence de nombreux autres indices d'un milieu steppique-héliophile permet de considérer ce taxon comme correspondant aux premières espèces.

Dans la zone pollinique b, un pic de Ranunculaceae (28\%) est enregistré (fig. 16). Leur étude microscopique et une comparaison avec des grains actuels permettent de penser qu'elles correspondent pour leur très grande majorité à des Ranunculus de la série Batrachium qui ne comprend que des espèces aquatiques ou de marais (Fournier, 1977). Le caractère isolé de ce pic implique que des conditions édaphiques particulières, strictement locales, ont favorisé l'essor de ces Ranunculaceae.

Plusieurs diagrammes polliniques provenant des PaysBas (Kolstrup, Wijmstra, 1977 ; Kolstrup, 1980) ou du nord de la Belgique (Haest et al., 1986) montrent également des pourcentages relativement élevés de Ranunculaceae type Batrachium au cours du Weichsélien 
ancien et du Pléniglaciaire moyen et supérieur, interprétés comme indicateurs d'une humidité édaphique plutôt que climatique. De même, des spectres polliniques ayant une forte représentation de renoncules aquatiques sont fréquemment rencontrés dans le Massif central et les Pyrénées au cours du Dryas ancien et du Dryas récent, où ils témoignent de la pureté et de la température très basse de l'eau, et dont la signification est strictement locale (Beaulieu et al., 1982, 1985 ; Reille et al., 1985 ; Reille, 1990b).

La zone pollinique $\mathrm{b}$ correspond à la présence continue de Polygonum et de spores trilètes, probablement Cryptogramma crispa, contrairement à la zone pollinique a. Certaines espèces de Polygonum (comme $P$. viviparum, $P$. alpinum) et Cryptogramma crispa sont des plantes arcto-alpines (Fournier, 1977; Fitter et al. 1991).

La couche stratigraphique 5 coïncide avec une nette augmentation de la courbe de concentration des Nymphaeaceae. L'accumulation et une assez longue stagnation de l'eau nécessaire à la formation d'un gley de toundra (Plaisance, Cailleux, 1958; Huetz de Lemps, 1994) semblent pouvoir expliquer le développement local de ces plantes.

Les spectres polliniques traduisent une végétation ouverte dominée par les Asteraceae type fenestré, Poaceae et Cyperaceae associés à des taxons xérophiles (Artemisia, Caryophyllaceae, Chenopodiaceae), héliophiles (Asteraceae type échinulé, Helianthemum, Thalictrum) et ubiquistes (Brassicaceae, Lamiaceae, Plantago, Ranunculaceae, Rosaceae). Seul Juniperus, avec des taux constants autour de $10 \%$, peut être considéré comme le représentant régional de la végétation ligneuse, les fréquences de Pinus ne pouvant correspondre qu'à des apports lointains.

Cette composition floristique diffère des steppes continentales où Pinus, Poaceae et Artemisia prédominent (Ozenda, 1964; Elhaï, 1968). Les spectres polliniques sont difficilement comparables à ceux traduisant des steppes méditerranéennes, formées principalement d'Artemisia et de Poaceae (Suc, 1984), ou des pelouses montagnardes, où Poaceae, plantes xérophiles et une grande richesse floristique d'ubiquistes sont associées à Pinus (Ablin, 1991). Le paysage asylvatique semble plus s'apparenter aux toundras nordiques à Poaceae et Cyperaceae, accompagnées de Juniperus, de quelques Betula, Salix et de rares Artemisia (Elhaï, 1968 ;
Huetz de Lemps, 1994 ; Ozenda, 1994). Des spectres semblables à ceux de Villiers-Adam (Asteraceae type fenestré associées à des Poaceae et Cyperaceae, suivies de Caryophyllaceae, (ihenopodiaceae, Thalictrum,...) se rencontrent dans une tourbière du Pays basque, à Le Moura (Oldfield, 1964). Cette composition floristique et la présence de deux espèces arcto-alpines, Polygonum cf. viviparum et Cryptogramma crispa, supportant des conditions climatiques très froides, ont été interprétées par l'auteur comme traduisant une végétation de type toundra.

La formation ouverte traduite par les spectres polliniques, caractérisée par la dominance de taxons héliophiles, l'absence d'arbres mésophiles, la faible représentation de Pinus, indique des températures faibles et peut être rapportée à une phase stadiaire du Pléistocène. Les différents composants de cette végétation sont stables tout le long de la séquence analysée et ne permettent donc pas d'entrevoir de possibles variations climatiques. Aucun élément ne permet de faire une attribution chronologique à base palynologique.

\section{Conclusion}

Malheureusement, aucune information pollinique concernant la fin du Saalien, l'Éémien, le Début Glaciaire weichsélien et le Pléniglaciaire supérieur n'a pu être obtenue sur ce site, à la différence d'autres sites de la région (Munaut, 1974, 1996 ; Leroi-Gourhan, 1988 ; Antoine et al., 1994, 1995b ; Sommé et al., 1994).

En revanche, des informations paléoenvironnementales ont été apportées concernant le Pléniglaciaire moyen, période pour laquelle très peu de données palynologiques sont disponibles en France septentrionale (Marcy et al., 1993). Ces données précisent l'organisation de la végétation lors du dépôt de la séquence sédimentaire. Elle correspond à une formation largement herbacée où les taxons héliophiles et xérophiles prédominent. Elle s'apparente aux toundras nordiques à Poaceae et Cyperaceae accompagnées de Juniperus et de rares Betula, Salix, Artemisia, et reflète un climat froid.

Deux épisodes indicateurs d'une humidité locale ont été par ailleurs mis en évidence au sommet de la séquence et ont été corrélés avec le sommet du premier sol de prairie arctique (couche 7 ) et le gley (couche j). 


\section{ÉTUDE DES MALACOFAUNES DU PETIT SAULE ET DU CHAMESSON}

\section{INTRODUCTION}

L'abondance des coquilles dans les formations loessiques est irrégulière. Elle dépend de la teneur en carbonate de calcium des sédiments et de la topographie des sites, les loess de versants de vallée et de bassins subsidents étant plus favorables à l'installation des mollusques que les loess de plateaux (Lozek, 1990, 2001). À Villiers-Adam, des échantillons tests d'environ un litre ont été prélevés dans toutes les unités stratigraphiques identifiées sur les sites du Chamesson et du Petit Saule. Les résultats de cette première approche ont permis de repérer la présence de restes coquilliers dans les lœess carbonatés du Saalien et l'ensemble de la séquence weichsélienne, les $B$, de sols bruns lessivés (Intrasaalien et Émien), très décarbonatés, sc sont révélés stériles.

\section{ÉCHANTILLONNAGE}

L'étude des malacofaunes de Villiers-Adam est basée sur un total de 121 échantillons, de 10 litres chacun, répartis sur 6 profils (fig. 21). Ils ont été pris en colonne continue suivant une maille de $10 \mathrm{~cm}$ d'épaisseur. Pour l'extraction des coquilles, le protocole de traitement suivi est celui établi par J.-J. Puisségur (1976).

\section{Les dépôts du Saalien}

Seuls les loess carbonatés du profil $C \emptyset$ sud du Chamesson se sont révélés fossilifères (fig. 22). Ces niveaux présentent un faciès lité à la base (couche 17), tandis que le sommet apparaît plus homogène (couche 16). Au total, 14 échantillons (Ml à MI4) proviennent de cette série.

\section{Le Début Glaciaire du Weichsélien}

Seule la séquence de la coupe $\mathrm{D}$ a été retenue pour un échantillonnage détaillé (fig. 15). Sept prélèvements (M1 à M7) recoupent le niveau de colluvions (couche 12) qui a livré l'occupation archéologique la plus importante (niveau N2), un sol gris-forestier (couche 11) archéologiquement stérile, et un sol isohu-
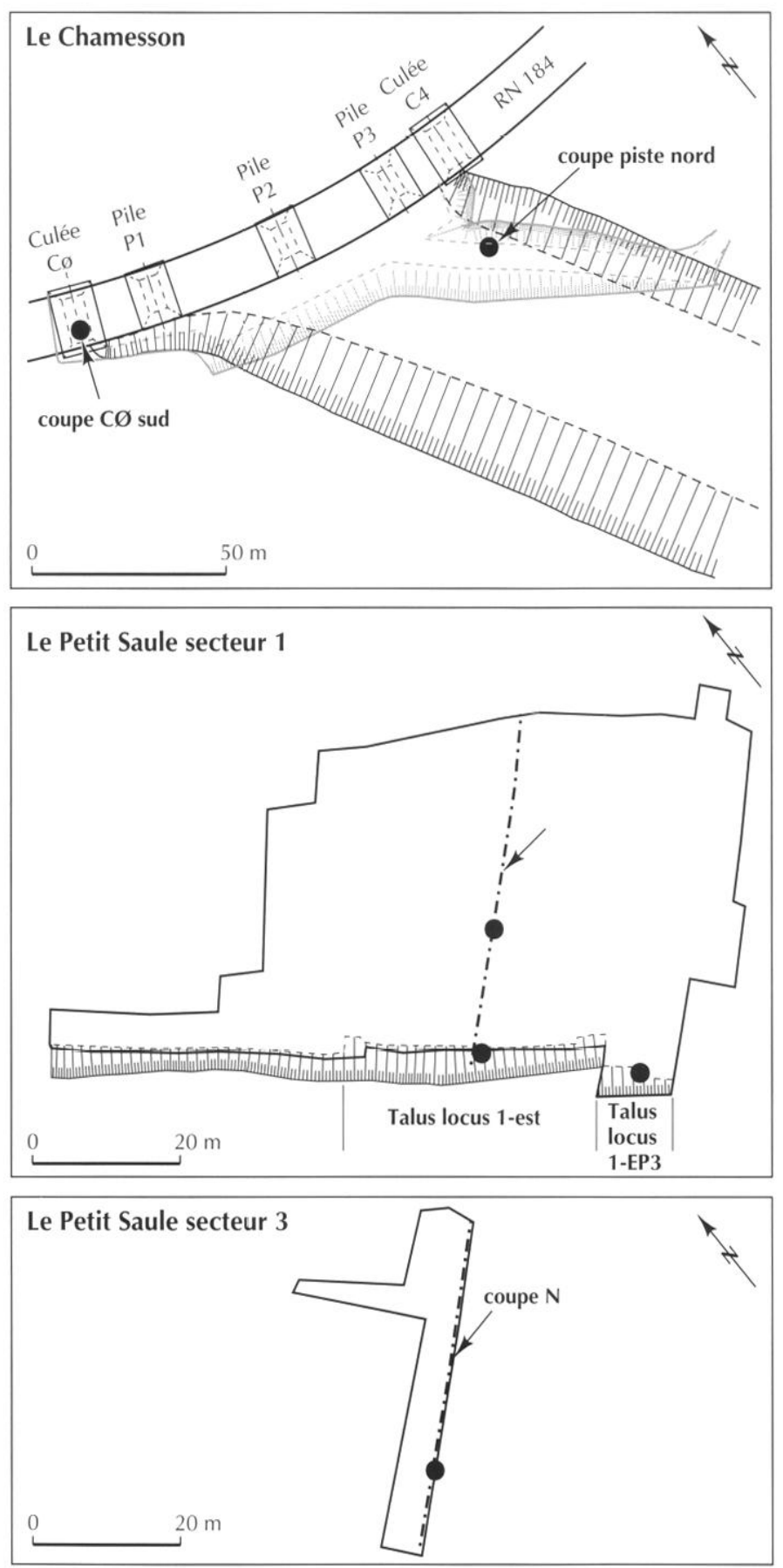

- - coupe stratigraphique

échantillonnage pour l'analyse malacologique

Fig. 21 - Plans des siles du Chamesson et du Petit Saule ì Villiers-Adam avec localisation des profils échantillonmés pour lëtude malacologique (dessin I. Raymond).

mique steppique (couche 10) qui contenait quelques artefacts épars (niveau N1). 


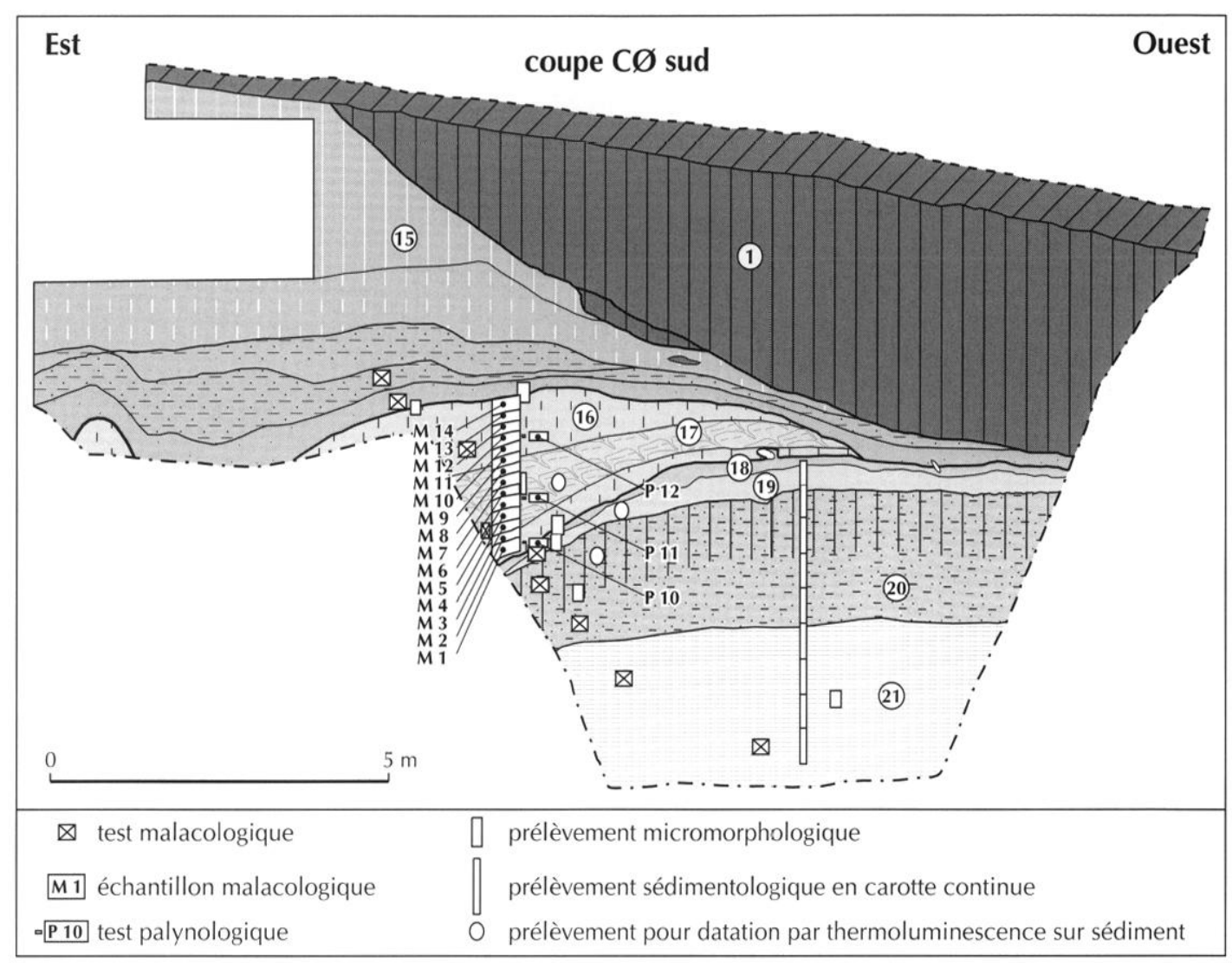

Fig. 22 - Villiers-Adam, le Chamesson, coupe CØ sud : localisation des tests et échantillons palynologiques et malacologiques, et des prélìvements sédimentologiques et datations TL-IRSL. (dessin J.J. Bahain et P. Raymond).

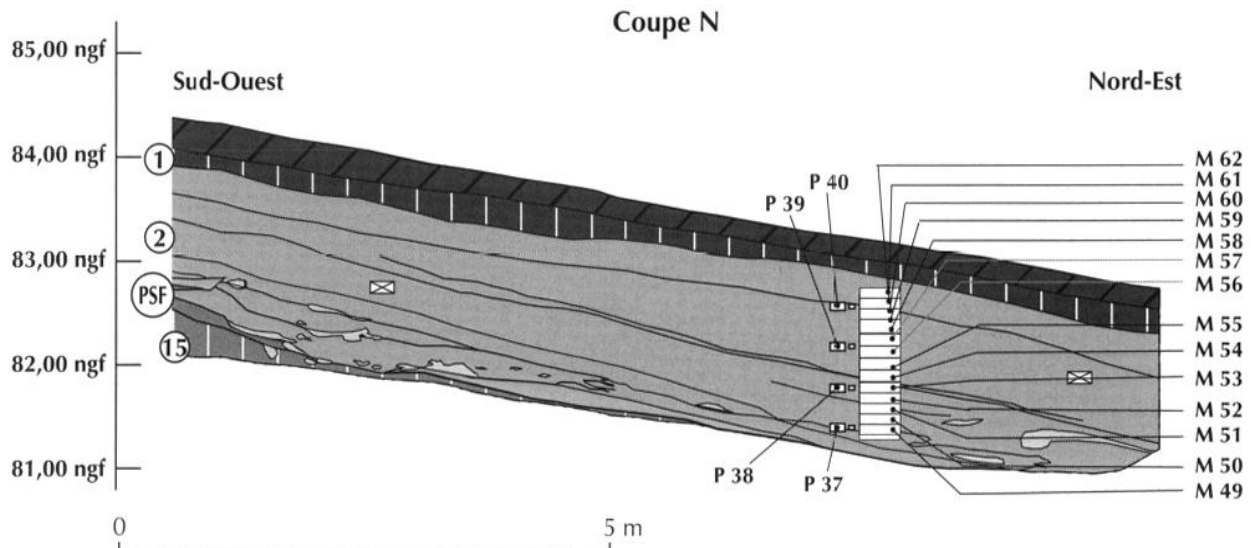

$\triangle$ test malacologique $\quad \mathbf{M 1}$ échantillon malacologique $\quad \square$ P1 test palynologique

Fig. 23 - Villiers-Adam, Le Petil Saule : séquence du Pléniglaciaire supérieur du Weichsélien avec position des prélèvements malacologiques, coupe N (dessin J.J. Bahain et P. Raymond). 


\section{Le Pléniglaciaire inférieur du Weichsélien}

La couche 9 de sable calcaire a été échantillonnée au Chamesson (M1 à M10, fig. 8) ainsi qu'au Petit Saule sur les coupes D (M8 à M13) et Talus locus 1-est (M12'). À la suite d'une erreur de numérotation entre deux phases de prélèvements sur le site du Petit Saule, les numéros 12 et 13 ont été attribués deux fois. Afin de préserver la simplicité des correspondances entre les différentes disciplines, cette numérotation est conservée sous la forme 12' et 13' pour les échantillons de la coupe Talus locus 1est (fig. 15).

\section{Le Pléniglaciaire moyen du Weichsélien}

La succession des couches 8 à 3 a été échantillonnée au Petit Saule sur les coupes Talus locus 1-est et Talus locus 1-EP3 (fig. 15), et au Chamesson sur la coupe Piste nord (fig. 8). Cet ensemble représente 33 échantillons pour Le Petit Saule et 32 pour Le Chamesson.

\section{Le Pléniglaciaire supérieur du Weichsélien}

Cette phase est représentée par la couche 2. Elle apparaît peu développée au Chamesson, au sommet de la coupe Piste nord (fig. 8), ce qui correspond aux 4 derniers prélèvements (M43 à M46). Au Petit Saule, en revanche, ces dépôts de lœess calcaires sont beaucoup plus dilatés et ont permis d'échantillonner une série de 14 prélèvements sur la coupe $\mathrm{N}$ (fig. 23). De M49 à M54, les lœess sont lités, puis ils deviennent homogènes dans leur partie supérieure (M55 à M62).

\section{LES MALACOFAUNES DU SAALIEN}

\section{Description des assemblages}

La liste des mollusques recensés révèle des associations extrêmement peu diversifiées, avec seulement 5 espèces (tabl. V). Le taxon dominant, Pupilla muscorum, caractéristique des milieux ouverts et secs, est actuellement répandu en Europe occidentale et centrale (Kerney et al., 1983). Également très commun aujourd'hui, Trichia hispida est un mollusque mésophile qui peut se développer dans une large gamme de biotopes, à l'exception des habitats extrêmement secs (Kerney et al., 1983). Les limaces, qui sont aussi bien représentées, ne
Tabl. V - I.iste des malacofaunes récoltées dans la séquence saalienne de la coupe CØ sud du Chamesson à Villiers-Adam.

\begin{tabular}{|c|c|c|c|c|c|c|}
\hline Échantillons & $\begin{array}{c}\text { Pupilla } \\
\text { muscorum }\end{array}$ & $\begin{array}{c}\text { Trichia } \\
\text { hispida }\end{array}$ & Limaces & $\begin{array}{c}\text { Succinea } \\
\text { oblonga }\end{array}$ & $\begin{array}{c}\text { Lymnaea } \\
\text { truncatula }\end{array}$ & Effectifs \\
\hline M14 & 202 & 27 & 15 & & & 244 \\
M13 & 325 & 39 & 25 & & 1 & 390 \\
M12 & 288 & 28 & 9 & & & 325 \\
M11 & 168 & 22 & 7 & & & 197 \\
M10 & 165 & 16 & 10 & & & 191 \\
M9 & 130 & 19 & 4 & & & 153 \\
M8 & 167 & 22 & 9 & & & 198 \\
M7 & 221 & 22 & 13 & & & 256 \\
M6 & 194 & 9 & 12 & & & 215 \\
M5 & 85 & 2 & 6 & & & 93 \\
M4 & 86 & 3 & 7 & & & 97 \\
M3 & 112 & 8 & 3 & & & 123 \\
M2 & 109 & 6 & 5 & & & 120 \\
M1 & 72 & 4 & 3 & & & 79 \\
\hline
\end{tabular}

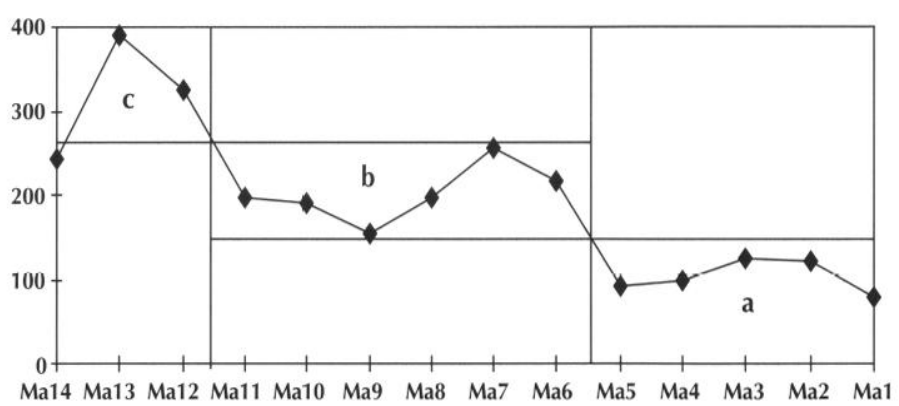

Fig. 24 - Courbe des effectifs malacologiques de la séquence saalienne du Chamesson (coupe CØ sud) à Villiers-Adam.

sont pas déterminables au rang spécifique. Leur présence indique cependant une humidité relative car elles n'offrent qu'une résistance limitée à la dessication. Les deux autres espèces de la liste, Succinea oblonga et Lymnaea truncatula, beaucoup plus hygrophiles, présentent des occurrences très ponctuelles. La principale variation au sein de ces faunes porte sur les effectifs qui augmentent progressivement de la base vers le sommet (fig. 24). Les 5 premiers échantillons sont compris dans une classe d'abondance " $\mathrm{a}$ " de 0 à 150 individus; la seconde division « $b$ " rassemble des associations dont les effectifs sont compris entre 150 et 250 éléments; enfin la classe " c » concerne les malacofaunes les plus riches, en sommet de séquence, qui peuvent compter jusqu'à près de 400 coquilles. 
La pauvreté de ces peuplements en termes de diversité spécifique implique un milieu difficile à coloniser, auquel peu d'espèces de mollusques sont capables de s'adapter. Par ailleurs, la progression régulière des quantités d'individus laisse supposer une certaine stabilité des conditions environnementales qui permet aux malacofaunes de développer leurs populations.

Les associations à petit nombre d'espèces mais riches en individus sont caractéristiques des dépôts loessiques de périodes glaciaires (Lozek, 1990). Les études de malacofaunes de séquences loessiques en Europe centrale (Lozck, 1964) et occidentale (Puisségur, 1976) ont permis de définir deux types d'associations de phase pléniglaciaire selon le degré d'humidité du milieu. Celles de la séquence saalienne de Villiers-Adam correspondent au type "Association à Pupilla " caractérisé par la dominance du genre Pupilla ( $P$. muscorum, P. loessica, $P$. alpicola, $P$. sterri) qui peut être accompagné par des proportions abondantes de Trichia hispida et de Succinea oblonga. Selon Puisségur (1976), ces faumes sont typiques de la steppe loxssique du Pléniglaciaire. Les faunes de la coupe $(\varnothing)$ sud présentent deux différences majeures par rapport au type. La première porte sur la présence d'une seule espèce de Pupilla: P. muscorum. Cet appaurrissement a déjà été souligné dans les séries loessiques du Bassin parisien par rapport à des séquences plus orientales d'Alsace ou de la région lyonnaise (Puisségur, 1976, 1978 ; Rousseau el al., 1990). La seconde particularité des associations saaliennes du Chamesson concerne l'absence de S. oblonga. Ce taxon étant très hygrophile (Kerney et al., 1983) sa défection dans les cortèges Pléniglaciaires de la coupe $(\varnothing)$ sud suggère un milieu assez sec.

\section{Analyse écologique des peuplements malacologiques}

\section{Diversité spécifique}

À la différence des faunes d'Europe centrale, voire d'Alsace et de la région lyonnaise, qui bénéficient de la proximité de massifs montagneux, celles du Bassin parisien se trouvent sur une aire géomorphologique monotone. Ceci explique que la faible diversité des malacofaumes loessiques s'accentue selon un gradient estouest. C'est en partant du même constat que s'organise la réflexion sur l'émigration des faunes lors des refroidissements et sur la biogéographie des associations pléniglaciaires (Rousseau, 1989a; Rousseau et al., 1990). La pauvreté spécifique observée dans les malacofaunes de Villiers-Adam caractérise aussi toutes les successions malacologiques loessiques de l'ouest de la France (Rousseau el al., 1990) ainsi que celles des îles AngloNormandes (Rousseau et Keen, 1989) et de GrandeBretagne (Kerney, 1971 ; Preece, 1990), et s'oppose aux faunes plus diversifiées récoltées dans les séquences losssiques d'Europe centrale (Lozek, 1972, 2001).

\section{Richesse spécifique}

Les espèces majoritaires dans les associations de la coupe $(\varnothing)$ sud sont des taxons résistants capables de supporter des températures extrêmes et des amplitudes importantes. Ceci confirme le caractère pléniglaciaire des conditions climatiques sous lesquelles se sont déposés les loess calcaires constituant les unités 16 et 17. En ce qui concerne l'humidité, la présence extrêmement sporadique de taxons très hygrophiles (un seul exemplaire de S. oblonga dans l'échantillon M4, et une Lymnaea truncatula dans le prélèvement M13) plaide en faveur d'une certaine sécheresse (tabl. V). Cependant cette appréciation se doit d'être pondérée au regard du développement constant des limaces dans toutes les associations de la série. En effet, le manque de coquille externe limite considérablement la résistance des limaces à la dessication et elles sont absentes des sites très secs (Boycott, 1934). Par ailleurs, la présence de limaces dans les associations loessiques n'est pas rare. Elles scmblent cependant être plus abondantes dans les sćrics de la zone atlantique, en Grande-Bretagne notamment (Kerney, 1971), qu'au sein des séquences plus continentales.

\section{Évolution des effectifs}

La seule variation notable dans les successions malacologiques de la coupe CØ sud porte sur les effectifs. Le calcul de deux indices écologiques, la diversité et l'équitabilité, a été effectué afin d'apprécier l'impact de l'augmentation des effectifs sur la structure des peuplements (Daget, 1979 ; Barbault, 1995).

Les valeurs de ces indices, calculées pour la série de la coupe ( $\varnothing$ sud (tabl. VI), sont traduites sous forme de courbes (fig. 25).

L'indice de diversité, ou indice de Shannon, varie entre des limites étroites (0,33 point d'amplitude), reflétant la grande homogénéité du peuplement malacologique tout au long de la série. L'indice d'équitabilité 
Tabl. VI - Valeurs des indices écologiques calculés pour la série. de la coupe CØ sud du Chamesson à Villiers-Adam.

\begin{tabular}{|c|c|c|}
\hline Échantillons & Diversité & Équitabilité \\
\hline M14 & 0,824 & 0,520 \\
M13 & 0,828 & 0,414 \\
M12 & 0,603 & 0,380 \\
M11 & 0,720 & 0,454 \\
M10 & 0,705 & 0,445 \\
M9 & 0,711 & 0,449 \\
M8 & 0,762 & 0,481 \\
M7 & 0,558 & 0,445 \\
M6 & 0,706 & 0,352 \\
M5 & 0,493 & 0,311 \\
M4 & 0,651 & 0,325 \\
M3 & 0,51 & 0,322 \\
M2 & 0,533 & 0,336 \\
M1 & 0,519 & 0,328 \\
\hline
\end{tabular}

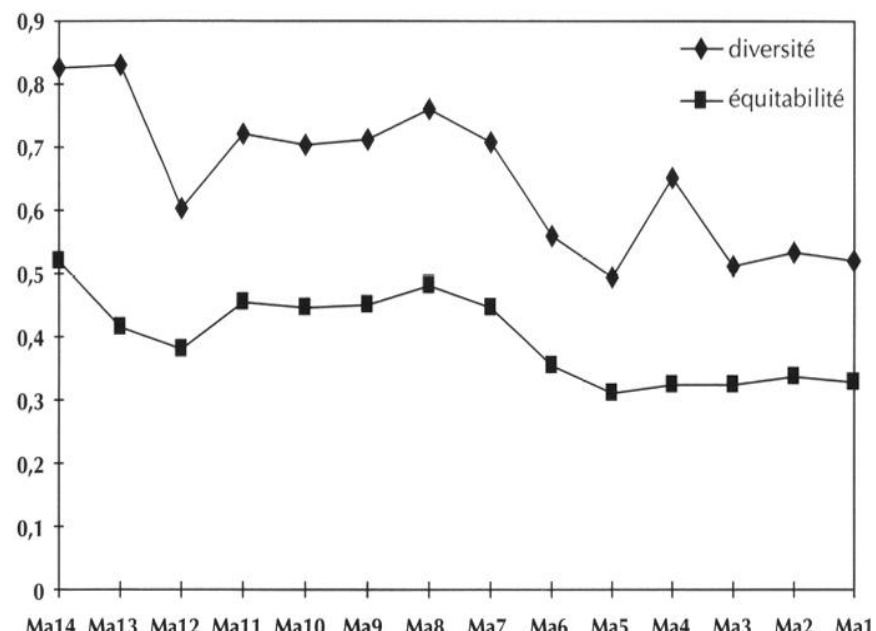

Fig. 25 - Courbes de variation des indices de diversité (I de Shannon) et d'équitabilité des associations malacologiques de la coupe $C \emptyset$ sud du Chamesson à Villiers-Adam.

reste stable dans des valeurs peu élevées, ce qui indique la forte dominance d'une ou plusieurs espèces au sein des associations. Ici le taxon prépondérant est P. muscorum (tabl. V). Le calcul de cet indice permet de démontrer quantitativement que l'augmentation progressive des effectifs ne fait pas varier le poids de cette espèce au sein des assemblages.

La constance de ces indices écologiques confirme l'interprétation d'une grande homogénéité du milieu et souligne qu'aucun changement majeur, environne- mental ou climatique, n'est intervenu au cours du dépôt de ces loess calcaires.

\section{LES SUCCESSIONS MALACOLOGIQUES DU WEICHSÉLIEN}

\section{Conservation des assemblages et évolution des effectifs}

Deux séries d'échantillons, correspondant aux sites du Petit Saule et du Chamesson, ont été étudiées. La séquence du Petit Saule est plus complète, car elle inclut les dépôts du début du Weichsélien. Celle du Chamesson a l'avantage d'avoir été échantillonnée sur une seule coupe en correspondance stricte avec les prélèvements des autres disciplines (fig. 8) et présente des enregistrements plus dilatés pour certains épisodes sédimentaires.

Sur les listes de faunes des séries weichséliennes (tabl. VII et VIII), deux taxons, Cecilioides acicula et Helicella itala, sont considérés comme intrusifs des niveaux holocènes. La première espèce, de mœurs souterraines, vit dans le sédiment où elle s'enfonce, à la faveur des racines ou autres bioturbations, à des profondeurs qui peuvent atteindre plusieurs dizaines de centimètres (Kerney et al., 1983). En Bourgogne, l'arrivée de ce taxon est située peu avant notre ère (Puisségur, 1976). La seconde espèce intrusive, Helicella itala, est recensée dans le centre du Bassin parisien au Tardiglaciaire (Limondin, Rousseau, 1991; Limondin, 1995; Limondin-Lozouet, 1997) et en Bourgogne vers l'époque subboréale (Puisségur, 1976). Les quelques spécimens récoltés au Petit Saule possèdent encore le periostracum, signe de leur modernité.

Les associations malacologiques du Weichsélien sont peu diversifiées, mais les effectifs varient beaucoup (tabl. VII et VIII). Ces oscillations, traduites sous forme de courbes (fig. 26 et 27), s'organisent en trois phases majeures.

Le premier ensemble qui couvre les sols du début du Weichsélien, le Pléniglaciaire inférieur et la première partie du Pléniglaciaire moyen moyen (niveaux 12 à 8 ) est caractérisé par des malacofaunes d'effectifs faibles à moyens (200 individus maximum). Les déficits d'effectifs sont à mettre en relation avec la décarbonatation des dépôts liée aux phénomènes de pédogenèse (voir tabl. I).

En revanche, dans le second ensemble, les malacofaunes sont quantitativement bien développées. Ceci 
Tabl. VII - Liste des malacofaunes weichséliennes du Petit Saule à Villiers-Adam: du bas vers le haut, suivant l'ordre chronologique des dépôts, les pavés du tableau correspondent aux coupes D, Talus locus 1-est, Talus locus 1-EP3 et $N$.

\begin{tabular}{|c|c|c|c|c|c|c|c|c|c|c|c|c|c|c|c|}
\hline Échantillons & $\begin{array}{l}\text { Pupilla } \\
\text { muscorum }\end{array}$ & $\begin{array}{l}\text { Trichia } \\
\text { hispida }\end{array}$ & Limaces & $\begin{array}{l}\text { Succinea } \\
\text { oblonga }\end{array}$ & $\begin{array}{l}\text { Vallonia } \\
\text { costata }\end{array}$ & $\begin{array}{l}\text { Vallonia } \\
\text { pulchella }\end{array}$ & $\begin{array}{l}\text { Vertigo } \\
\text { pygmaea }\end{array}$ & $\begin{array}{l}\text { Cochlicopa } \\
\text { lubrica }\end{array}$ & $\begin{array}{l}\text { Helicopsis } \\
\text { striata }\end{array}$ & $\begin{array}{l}\text { Arianta } \\
\text { arbustorum }\end{array}$ & $\begin{array}{l}\text { Nesovitrea } \\
\text { hammonis }\end{array}$ & $\begin{array}{l}\text { Amiger } \\
\text { crista }\end{array}$ & Effectifs & $\begin{array}{l}\text { Cecilioides } \\
\text { acicula }\end{array}$ & $\begin{array}{l}\text { Helicella } \\
\text { itala }\end{array}$ \\
\hline M62 & 12 & 22 & 3 & 2 & 1 & 1 & & & & & & & 23 & 200 & \\
\hline M61 & 19 & 4 & 2 & 12 & 1 & & & & & & & & 38 & 96 & \\
\hline M60 & 8 & 3 & 3 & 10 & 1 & 2 & & & & & & 1 & 28 & 62 & \\
\hline M59 & 18 & 2 & 3 & 12 & 1 & 1 & 1 & & & & & & 38 & 62 & \\
\hline M58 & 7 & 2 & 2 & 22 & & 2 & 1 & & & & & & 36 & 20 & \\
\hline M57 & 16 & 1 & & 16 & & 2 & & & & & & & 35 & 18 & \\
\hline M56 & 5 & 1 & 1 & 8 & & & & & & & & & 15 & 10 & 1 \\
\hline M55 & 14 & 1 & & 10 & & 2 & & & & & & & 27 & 7 & \\
\hline M54 & 14 & 1 & 2 & 18 & & & & & & & & & 35 & 1 & \\
\hline M53 & 11 & 1 & & 6 & & & & & & & & & 18 & 3 & \\
\hline M52 & 6 & 1 & 1 & 34 & & & & & & & & & 42 & & \\
\hline M51 & 20 & 1 & 4 & 122 & & & & & & & & & 147 & 1 & \\
\hline M50 & 40 & 3 & 9 & 44 & & & & & & & & & 96 & 1 & \\
\hline M49 & 61 & 5 & 7 & 53 & & & & & & & & & 126 & & \\
\hline M48 & 232 & 7 & 14 & 30 & & & & & & & & & 283 & 38 & \\
\hline M47 & 201 & 8 & 8 & 19 & 1 & & & & & & & & 237 & 29 & 2 \\
\hline M46 & 130 & 4 & 6 & 24 & & & & & & & & & 164 & 20 & 2 \\
\hline M45 & 110 & 10 & 13 & 7 & & & & & & & & & 140 & 13 & \\
\hline M44 & 73 & 5 & 7 & 8 & & & & & & & & & 93 & 3 & \\
\hline M43 & 87 & 5 & 5 & 15 & 1 & & & & & & & & 133 & 3 & \\
\hline $\mathrm{M} 42$ & 97 & 9 & 8 & 21 & & & & & & & & & 135 & 7 & \\
\hline M41 & 141 & 20 & 16 & 25 & & & & & & & & & 202 & 2 & \\
\hline $\mathrm{M} 40$ & 135 & 8 & 32 & 22 & & & & & & & & & 197 & 5 & \\
\hline M39 & 288 & 15 & 28 & 19 & 1 & & & & & & 1 & & 372 & 1 & \\
\hline M38 & 792 & 30 & 25 & 53 & & & & & & & & & 900 & 1 & \\
\hline M37 & 470 & 17 & 17 & 53 & 1 & & & & & & & & 558 & & \\
\hline M36 & 110 & 16 & 38 & 17 & 1 & & & & & & & & 182 & & \\
\hline M35 & 142 & 26 & 52 & 20 & 1 & & & & & 1 & & & 242 & & \\
\hline M34 & 157 & 15 & 36 & 23 & 2 & & & & & & & & 233 & & \\
\hline M33 & 219 & 20 & 72 & 18 & & 1 & & & & & & & 330 & & \\
\hline M32 & 201 & 22 & 73 & 19 & 2 & & & & & & & & 317 & & \\
\hline M28 & 128 & 13 & 19 & 8 & & & & & & & & & 168 & 40 & 1 \\
\hline M27 & 153 & 13 & 5 & 1 & & & & & & & & & 172 & 20 & 1 \\
\hline M26 & 144 & 13 & 2 & 1 & & & & & & & & & 160 & 5 & 2 \\
\hline M25 & 81 & 12 & 4 & 1 & & & & & & & & & 98 & 16 & 1 \\
\hline M24 & 52 & 8 & 4 & & & & & & & & & & 64 & 3 & 1 \\
\hline M23 & 36 & 5 & 1 & & & & & & & & & & 42 & 2 & \\
\hline M22 & 40 & 4 & 2 & & & & & & & & & & 46 & 4 & \\
\hline M21 & 63 & 7 & 3 & & & & & & & & & & 73 & & \\
\hline M20 & 51 & 4 & 2 & & & & & & & & & & 57 & & \\
\hline M19 & 38 & 15 & 4 & 3 & & & & & & & & & 61 & & \\
\hline M18 & 68 & 7 & 12 & 24 & 2 & & & & & & & & 113 & & \\
\hline M17 & 85 & 17 & 12 & 3 & 1 & & & & & & & & 118 & & \\
\hline M16 & 142 & 10 & 22 & 3 & 1 & & & & & & & & 178 & & \\
\hline M15 & 11 & 11 & & 1 & & & & & & & & & 112 & & \\
\hline M14 & 52 & 4 & 3 & & & & & & & & & & 59 & & \\
\hline M13' & 38 & 14 & & & & & & & & 1 & 52 & & & & \\
\hline M12' & 30 & 14 & 5 & 1 & & & & & & & & & 50 & & \\
\hline M13 & 87 & 26 & 8 & & 5 & 1 & & & & & & & 127 & 2 & \\
\hline M12 & 114 & 29 & 15 & & 6 & & 1 & & & 1 & & & 167 & & \\
\hline M11 & 81 & 28 & 12 & & 6 & & & & 1 & 1 & & & 128 & & \\
\hline M10 & 77 & 27 & 5 & & 4 & & & & & 1 & & & 112 & & \\
\hline M9 & 89 & 22 & 9 & & 2 & & & 1 & & 1 & & & 124 & & \\
\hline M8 & 70 & 22 & 5 & & & & & & & 1 & & & 98 & & \\
\hline M7 & 30 & 17 & 2 & 1 & 2 & & & & & 1 & & & 53 & & \\
\hline M6 & 28 & 16 & & 1 & 1 & & & & & 1 & & & 47 & & \\
\hline M5 & 14 & 13 & & 1 & & & & & & & & 1 & 29 & & \\
\hline M4 & 20 & 10 & 2 & & & & & & & & & & 32 & & \\
\hline M3 & 13 & 5 & 1 & & & & & & & & & & 20 & & \\
\hline M2 & 6 & 9 & 1 & & & & & & & & & & 16 & & \\
\hline M1 & 1 & 3 & 1 & & & & & & & & & & 5 & & \\
\hline
\end{tabular}


Tabl. VIII - Liste des malacofaunes weichséliennes de la coupe Piste nord du Chamesson à Villiers-Adam. Les prélèvements sont présentés suivant l'ordre chronologique des dépôts du plus ancien en bas (MI) au plus récent en haut (M46).

\begin{tabular}{|c|c|c|c|c|c|c|c|c|c|}
\hline Échantillons & $\begin{array}{l}\text { Pupilla } \\
\text { muscorum }\end{array}$ & $\begin{array}{l}\text { Trichia } \\
\text { hispida }\end{array}$ & Limaces & $\begin{array}{l}\text { Succinea } \\
\text { oblonga }\end{array}$ & $\begin{array}{l}\text { Vallonia } \\
\text { costata }\end{array}$ & $\begin{array}{l}\text { Vallonia } \\
\text { pulchella }\end{array}$ & $\begin{array}{l}\text { Vertigo } \\
\text { pygmaea }\end{array}$ & Effectifs & $\begin{array}{l}\text { Cecilioides } \\
\text { acicula }\end{array}$ \\
\hline M46 & 66 & 1 & 8 & 48 & 1 & 1 & & 125 & 22 \\
\hline M45185 & 3 & 9 & 97 & 1 & & & 1 & 296 & 45 \\
\hline M44 & 259 & 1 & 8 & 151 & 1 & & & 420 & 24 \\
\hline M43 & 71 & 2 & 11 & 21 & 1 & & & 106 & 13 \\
\hline M42 & 41 & 1 & 2 & 20 & 1 & & & 65 & 18 \\
\hline M41 & 32 & 3 & 3 & 14 & & & & 52 & 15 \\
\hline M40 & 123 & 9 & 12 & 69 & 1 & & & 214 & 7 \\
\hline M39 & 141 & 13 & 11 & 27 & 1 & & & 193 & 7 \\
\hline M38 & 80 & 7 & 15 & 27 & 1 & & & 130 & 2 \\
\hline M37 & 150 & 6 & 19 & 29 & 2 & & & 208 & 2 \\
\hline M36 & 213 & 12 & 8 & 41 & 1 & & & 275 & \\
\hline M35 & 137 & 12 & 6 & 43 & & & & 198 & 2 \\
\hline M34 & 72 & 8 & 1 & 30 & & & & 111 & 1 \\
\hline M33 & 20 & 2 & 3 & 14 & 1 & & & 40 & 2 \\
\hline M32 & 108 & 1 & & 49 & & & & 158 & \\
\hline M31 & 535 & 1 & 5 & 288 & & & & 829 & \\
\hline M30 & 142 & 5 & 9 & 47 & 1 & & & 204 & \\
\hline M29 & 253 & 1 & 39 & 19 & & & & 312 & \\
\hline M28 & 287 & 2 & 47 & 20 & & & & 356 & \\
\hline M27 & 313 & 2 & 49 & 17 & & & & 381 & \\
\hline M26 & 258 & 24 & 39 & 20 & & & & 341 & \\
\hline M25 & 190 & 15 & 29 & 9 & & & & 243 & \\
\hline M24 & 254 & 53 & 27 & 4 & & & & 338 & \\
\hline M23 & 397 & 71 & 26 & 2 & & & & 496 & \\
\hline M22 & 199 & 14 & 24 & 3 & & & & 240 & \\
\hline M21 & 147 & 14 & 18 & & & & & 169 & \\
\hline M20 & 86 & 8 & 3 & 1 & & & & 98 & \\
\hline M19 & 33 & 5 & & & & & & 38 & \\
\hline M18 & 30 & 5 & 1 & & & & & 36 & \\
\hline M17 & 43 & 8 & 1 & 1 & & & & 53 & \\
\hline M16 & 51 & 8 & 3 & 2 & & & & 64 & \\
\hline M15 & 31 & 5 & 3 & & & & & 39 & \\
\hline M14 & 47 & 19 & 2 & 2 & & & & 70 & \\
\hline M13 & 44 & 11 & 6 & & & & & 61 & \\
\hline M12 & 148 & 27 & 22 & & 1 & & 1 & 199 & \\
\hline M11 & 179 & 19 & 14 & 1 & 1 & & & 214 & \\
\hline M10 & 96 & 16 & 5 & & & & & 117 & \\
\hline M9 & 78 & 12 & 7 & 2 & 2 & & 1 & 102 & \\
\hline M8 & 57 & 13 & 6 & 1 & & & 1 & 78 & \\
\hline M7 & 69 & 11 & 3 & & & & & 83 & \\
\hline M6 & 43 & 9 & 1 & & & & & 53 & \\
\hline M5 & 45 & 11 & & 1 & & & & 57 & \\
\hline M4 & 42 & 13 & 2 & & & & & 57 & \\
\hline M3 & 27 & 12 & & 1 & & & & 40 & \\
\hline M2 & 19 & 3 & & & & & & 22 & \\
\hline M1 & 14 & 4 & & & & & 1 & 19 & \\
\hline
\end{tabular}

concerne le Pléniglaciaire moyen à partir des sols de prairie arctique (niveaux 7 et 6 ), jusqu'à la base des dépôts du Pléniglaciaire supérieur (niveau 3).

Une troisième division se marque au sommet de la série du Petit Saule pour les faunes du Pléniglaciaire supérieur de la coupe $\mathrm{N}$, qui apparaissent très pauvres. $\mathrm{Si}$ les taux de carbonate de calcium sont élevés au cours de cette période, en revanche ceux du carbone organique, liés au développement de la végétation, sont bas (voir p. 49). Cette faible densité de la couverture végétale dont les mollusques sont dépendants pour leur nourriture et leur habitat influe directement sur l'expansion quantitative des peuplements malacologiques.

\section{Composition des peuplements}

Afin d'apprécier l'occurrence et les variations des effectifs de chaque espèce, des diagrammes malaco- 


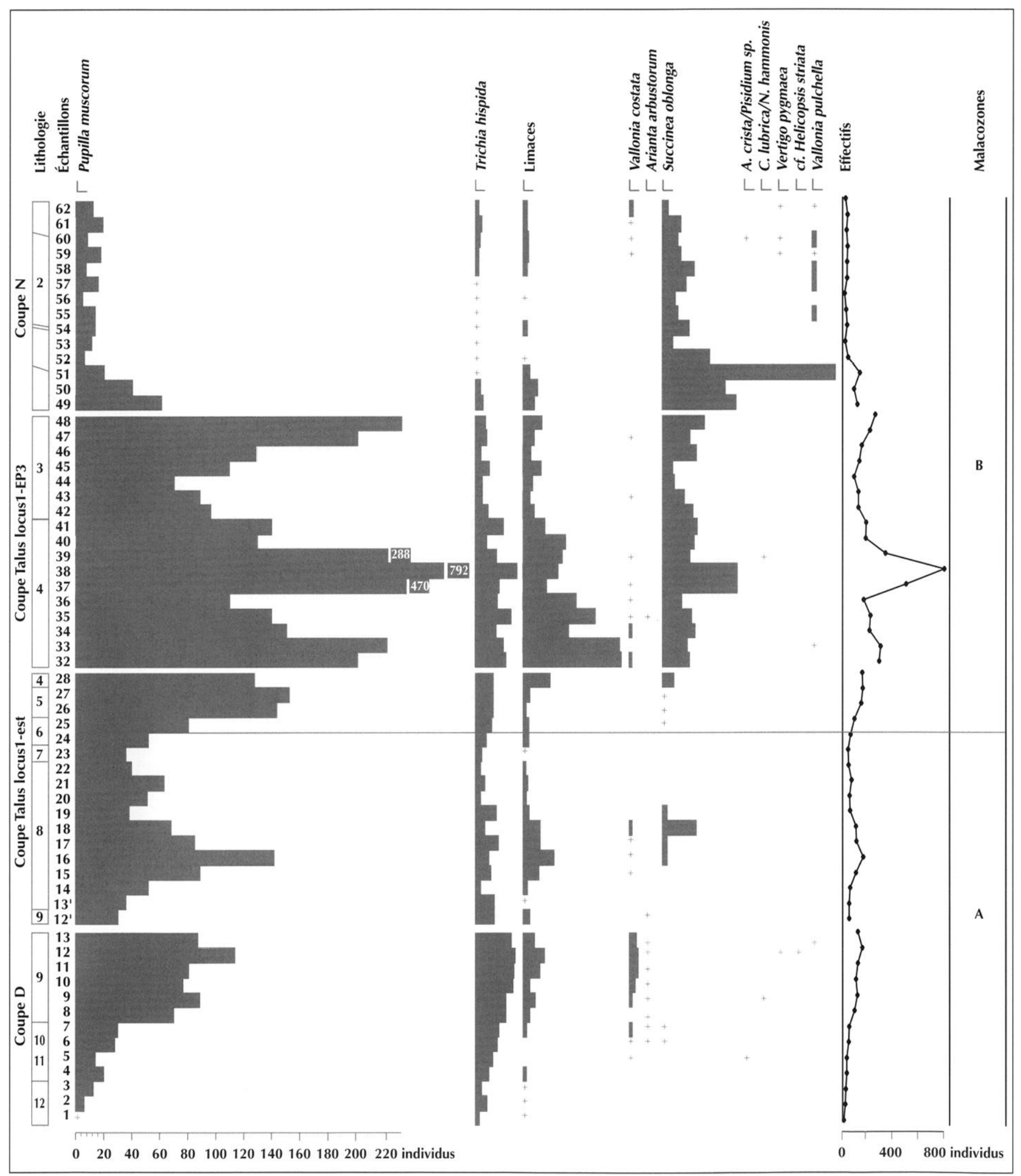

Fig. 26 - Diagramme malacologique de la séquence weichsélienne du Petil Saule à Villiers-Adam. Chaque croix représente un seul individu (dessin N. Limondin). 
logiques ont été tracés pour les séries weichséliennes du Petit Saule (fig. 26) et du Chamesson (fig. 27).

De façon globale, les associations weichséliennes de Villiers-Adam, tout comme celles du Saalien, se rattachent au type "Association à Pupilla " caractéristique des milieux de steppe lossique. P. muscorum, T. hispida et les limaces sont bien représentées dans toutes les unités. La distribution de $S$. oblonga est intéressante et permet de définir deux biozones. Dans la malacozone A, sur les deux séries, elle est sporadique, voire absente, du Début
Glaciaire jusqu'à la couche 8 incluse. Dans la malacozone B, à partir des sols de type arctique (niveaux 7 et 6 ), $S$. oblonga devient constante et présente ensuite deux pics de développement, l'un dans le gley (couche 5) sur le site du Chamesson, l'autre à la base des dépôts du Pléniglaciaire supérieur sur les deux séries (fig. 26 et 27). S. oblonga est un mollusque de milieux humides que l'on trouve aujourd'hui dans des habitats à végétation éparse (zone inondable des rivières, marécages). Elle est caractéristique des surfaces dénudées et

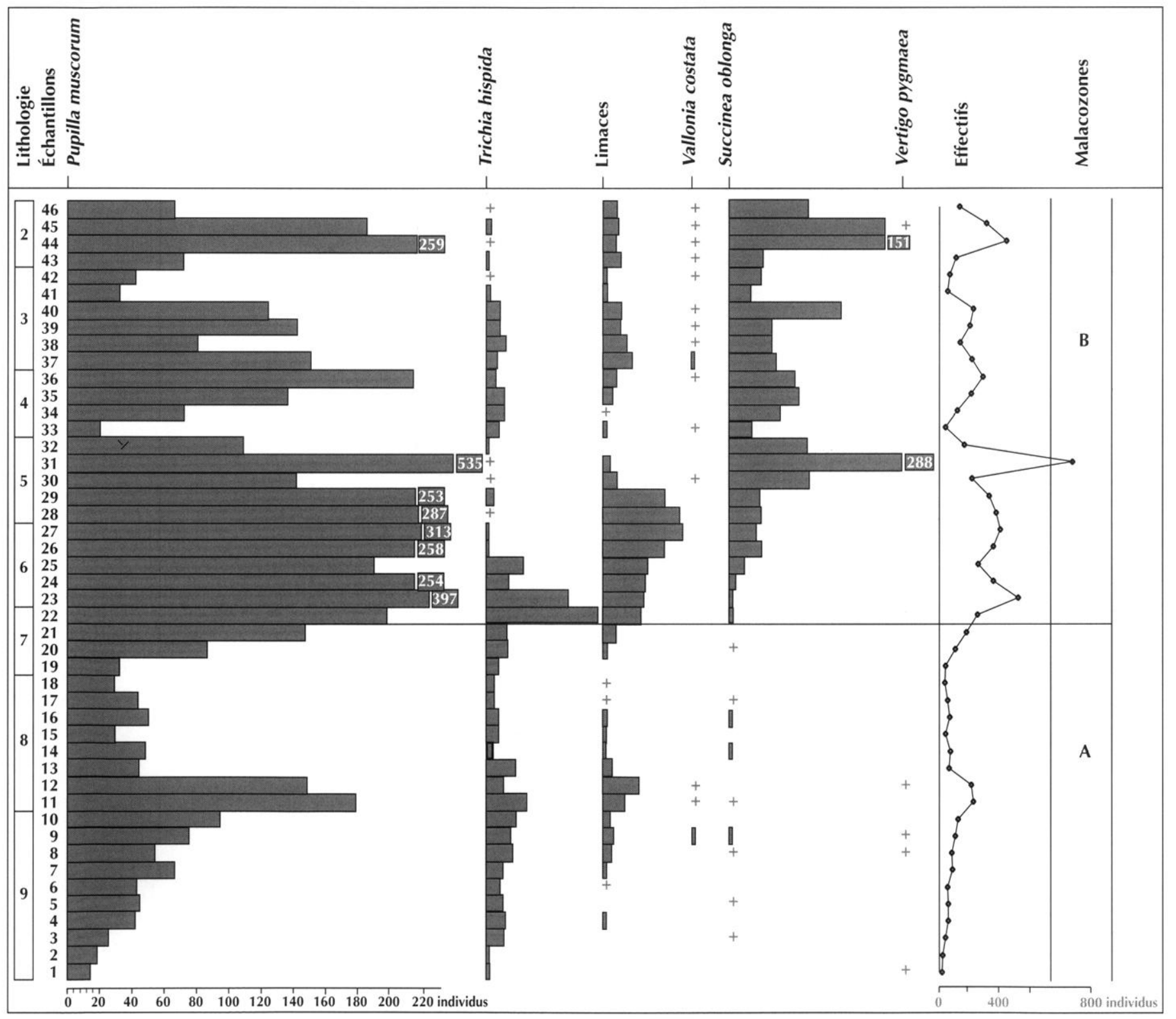

Fig. 27 - Diagramme malacologique de la séquence weichsélienne du Chamesson à Villiers-Adam. Chaque croix représente un seul individu (dessin N. Limondin). 
Tabl. $\mathbf{X}$ - Liste des espèces et prélèvements malacologiques de VilliersAdam dont les contributions relatives à l'inertie du premier plan factoriel sont supérieures à la moyenne (9,09 pour les espèces; 0,83 pour les prélèvements).

\begin{tabular}{|c|c|c|c|c|}
\hline \multicolumn{2}{|c|}{ Contribution à l'inertie totale } & \multicolumn{2}{|c|}{$\begin{array}{c}\text { Axe } 1 \\
28,60 \%\end{array}$} & \multirow{2}{*}{$\begin{array}{r}\text { Axe 2 } \\
17,30 \% \\
+\end{array}$} \\
\hline \multicolumn{2}{|l|}{ Espèces } & + & - & \\
\hline \multicolumn{2}{|l|}{ Trichia hispida } & 17,2 & & \\
\hline \multicolumn{2}{|l|}{ Succinea oblonga } & & 64,3 & \\
\hline \multicolumn{2}{|l|}{ Vallonia costata } & & & 26,9 \\
\hline \multicolumn{2}{|l|}{ Vertigo pygmaea } & & & 26,2 \\
\hline \multicolumn{2}{|l|}{ Vallonia pulchella } & & & 22 \\
\hline \multicolumn{2}{|l|}{ Arianta arbustorum } & & & 12,7 \\
\hline \multicolumn{2}{|l|}{ Prélèvements } & + & - & + \\
\hline \multirow[t]{7}{*}{ Saalien Chamesson Coupe CØ sud } & M7 & 0,9 & & \\
\hline & M8 & 0,9 & & \\
\hline & M9 & 1 & & \\
\hline & M11 & 0,9 & & \\
\hline & M12 & 0,9 & & \\
\hline & M13 & 1,1 & & \\
\hline & M14 & 0,9 & & \\
\hline \multirow[t]{16}{*}{ Weichsélien Chamesson Piste nord } & M4 & 0,8 & & \\
\hline & M6 & 0,8 & & \\
\hline & M7 & 0,8 & & \\
\hline & M21 & 0,8 & & \\
\hline & M31 & & 2,8 & \\
\hline & M32 & & 2 & \\
\hline & M33 & & 0,8 & \\
\hline & M40 & & 0,9 & \\
\hline & M42 & & 1,3 & \\
\hline & M44 & & 2,2 & \\
\hline & M45 & & 1,6 & 0,9 \\
\hline & M46 & & 2,8 & 1,1 \\
\hline & M1 & & & 3,1 \\
\hline & M8 & & & 0,9 \\
\hline & M9 & & & 2 \\
\hline & M12 & & & 1,5 \\
\hline \multirow[t]{10}{*}{ Weichsélien PS Coupe D } & M2 & 0,8 & & \\
\hline & M4 & 0,8 & & \\
\hline & M8 & 1,9 & & \\
\hline & M6 & 1 & & 2 \\
\hline & M7 & 1,1 & & 1,6 \\
\hline & M9 & 3,5 & & 3,7 \\
\hline & M10 & 1,7 & & 2,4 \\
\hline & M11 & 2,9 & & 13,2 \\
\hline & M12 & 1,2 & & 9,6 \\
\hline & M13 & & & 5,7 \\
\hline \multirow[t]{2}{*}{ Weichsélien PS Locus1-est } & M12' & 1,7 & & \\
\hline & M13' & 1,9 & & \\
\hline \multirow[t]{8}{*}{ Weichsélien PS Coupe N } & M49 & & 1 & \\
\hline & M50 & & 1,2 & \\
\hline & M51 & & 2,8 & \\
\hline & M52 & & 3 & \\
\hline & M54 & & 1,8 & \\
\hline & M55 & & 3,2 & 1,2 \\
\hline & M57 & & 3,2 & 1,2 \\
\hline & M58 & & 4,6 & 6,3 \\
\hline
\end{tabular}

boueuses. Sa répartition actuelle est locale dans les îles Britanniques et le sud de la Scandinavie, mais elle est commune dans le reste de l'Europe (Kerney et al., 1983). L'occurrence et la fréquence de cette espèce hygrophile sont un indicateur des fluctuations des conditions d'humidité sur le site de Villiers-Adam.

Les taxons dominants des assemblages du Weichsélien sont des eurythermes. Le cortège secondaire des associations est composé d'espèces moins résistantes aux forts contrastes thermiques, dont l'apparition peut signaler des phases de changement des températures. Toutefois, l'occurrence des espèces sténothermes dépasse rarement l'individu unique. Seule $V$. costata, qui présente une tolérance plus importante aux variations thermiques, a parfois des fréquences un peu plus élevées. Il faut sans doute relier la présence de ces espèces à des épisodes au cours desquels les écarts de température sont de moindre amplitude, plutôt qu'à des réchauffements au sens strict. Les cortèges les plus diversifiés d'espèces secondaires sont observés à la base de la série du Petit Saule (Début Glaciaire et Pléniglaciaire inférieur) et à son sommet pendant le Pléniglaciaire supérieur. Durant le Pléniglaciaire moyen, seule $V$. costata subsiste de manière sporadique. Sur la coupe du Chamesson, le cortège secondaire est encore plus pauvre et ne compte que deux taxons ( $V$. costata et Vertigo pygmaea) qui apparaissent aux transitions Pléniglaciaire inférieur/ moyen et Pléniglaciaire moyen/supérieur.

\section{VARIATIONS DES PARAMÈTRES CLIMATIQUES EXPRIMÉES PAR LES MALACOFAUNES}

Les données malacologiques de Villiers-Adam ont été traitées par analyse factorielle des correspondances, afin d'obtenir une quantification relative des paramètres climatiques (Rousseau, 1990).

L'analyse factorielle a porté sur 121 prélèvements et 11 espèces (les espèces aquatiques, Armiger crista, Lymnaea truncatula et Pisidium sp. n'ont pas été prises en compte, leur présence très ponctuelle pouvant être accidentelle). La contribution à l'inertie totale du nuage du premier plan factoriel est élevée : 45,9\%. La moyenne de la contribution relative pour les prélèvements est de 0,83 et de 9,09 pour les espèces. La liste des points à forte contribution est donnée dans le tableau IX. Seuls trois pôles apparaissent bien caractérisés sur le premier plan factoriel (tabl. IX, fig. 28). Sur l'axe 1 s'opposent un taxon franchement hygrophile, S. oblonga, et une espèce mésophile, T. hispida, qui définissent ainsi un gradient d'humidité. Vers le pôle positif de l'axe 2 se regroupent des taxons sténothermes auxquels 


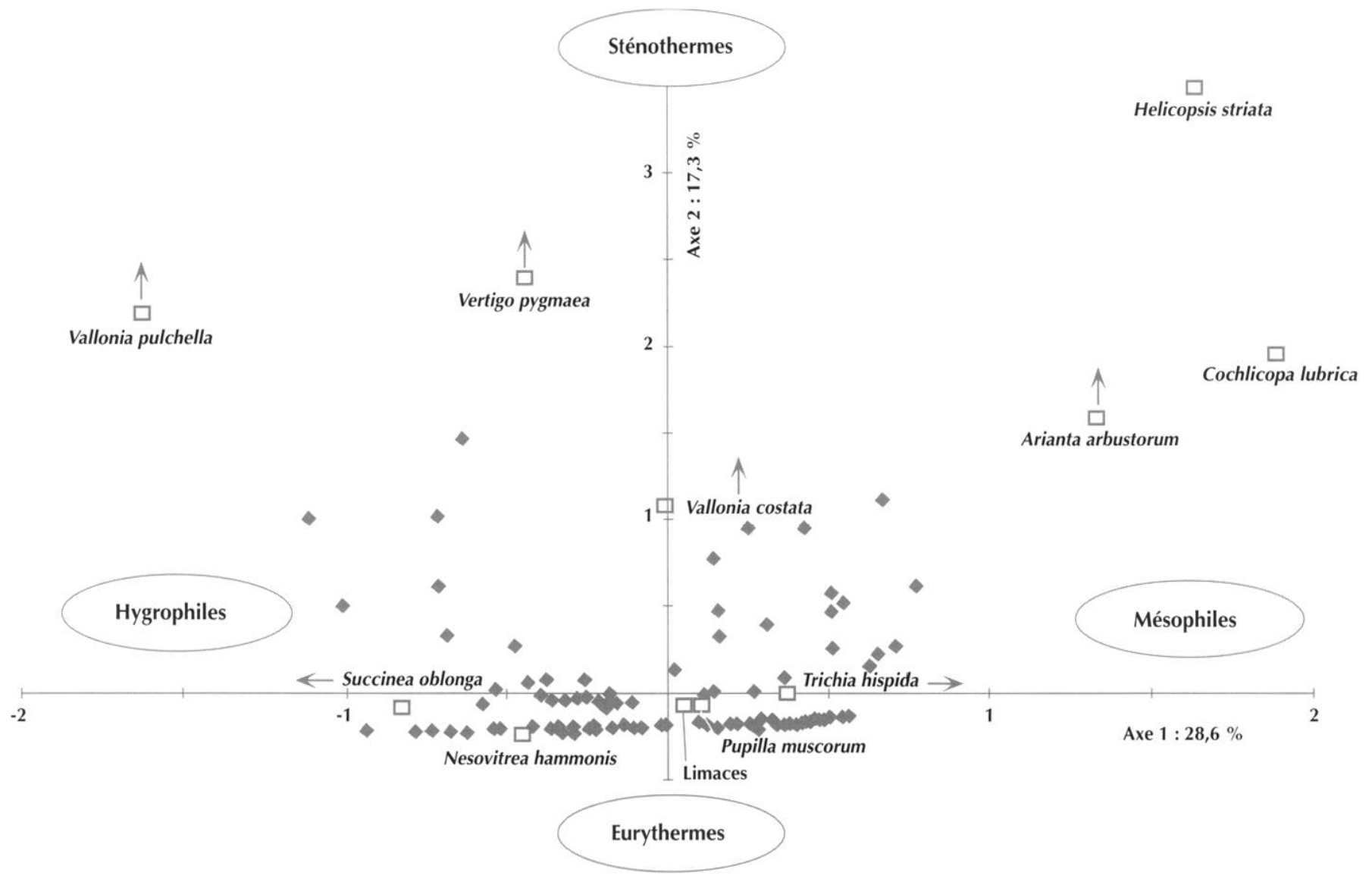

Fig. 28 - Projection des espèces et des prélèvements des malacofaunes de Villiers-Adam sur le premier plan factoriel.

Les flèches matérialisent les plus fortes contributions relatives des espèces aux axes.

s'ajoute $V$ costata dont le caractère eurytherme peu accusé a déjà été signalé. Aucune espèce ni prélèvement ne présente de contribution relative supérieure à la moyenne autour du pôle négatif de l'axe 2. Cependant, tous les taxons eurythermes sont situés dans la partie négative de cet axe (fig. 28). Ainsi, le deuxième axe peut être interprété comme représentatif d'une certaine variabilité des températures, mais il est moins pertinent que le premier.

La projection des prélèvements sur le premier plan factoriel est illustrée (fig. 29). Chaque schéma représente un niveau stratigraphique. Les associations de la coupe Piste nord du Chamesson sont figurées sous forme de carrés, celles des profils du Petit Saule sont représentées par des triangles. Ces projections permettent d'apprécier la cohérence des données malacologiques recueillies au Chamesson et au Petit Saule, les ensembles de prélèvements des deux sites se recoupent. Seul le nuage de la couche 5 (fig. 29, F) montre une différence entre les deux gisements qui porte sur le gradient d'humidité. Au Chamesson, les malacofaunes enregistrent des conditions nettement plus humides qu'au Petit Saule. Cependant, il faut souligner que sur la coupe Talus locus 1-est, le gley est peu épais $(20 \mathrm{~cm})$ et n'a fait l'objet que de deux échantillons (fig. 15).

Les variations des paramètres climatiques sont également exprimées sous forme de courbes (fig. 30) et comparées aux résultats obtenus sur les cortèges malacologiques du site d'Achenheim, Bas-Rhin (Puisségur, 1978 ; Rousseau, 1987, 1989b ; Rousseau, Puisségur, 1990). Toutefois, pour apprécier correctement les oscillations climatiques traduites par les associations malacologiques de Villiers-Adam, il convient de préciser certaines données.

L'axe 1, qui apparaît bien caractérisé sur ses deux pôles par des espèces et par des assemblages, exprime des variations relatives de l'humidité. Cependant, il semble intéressant de souligner que, entre les deux types 


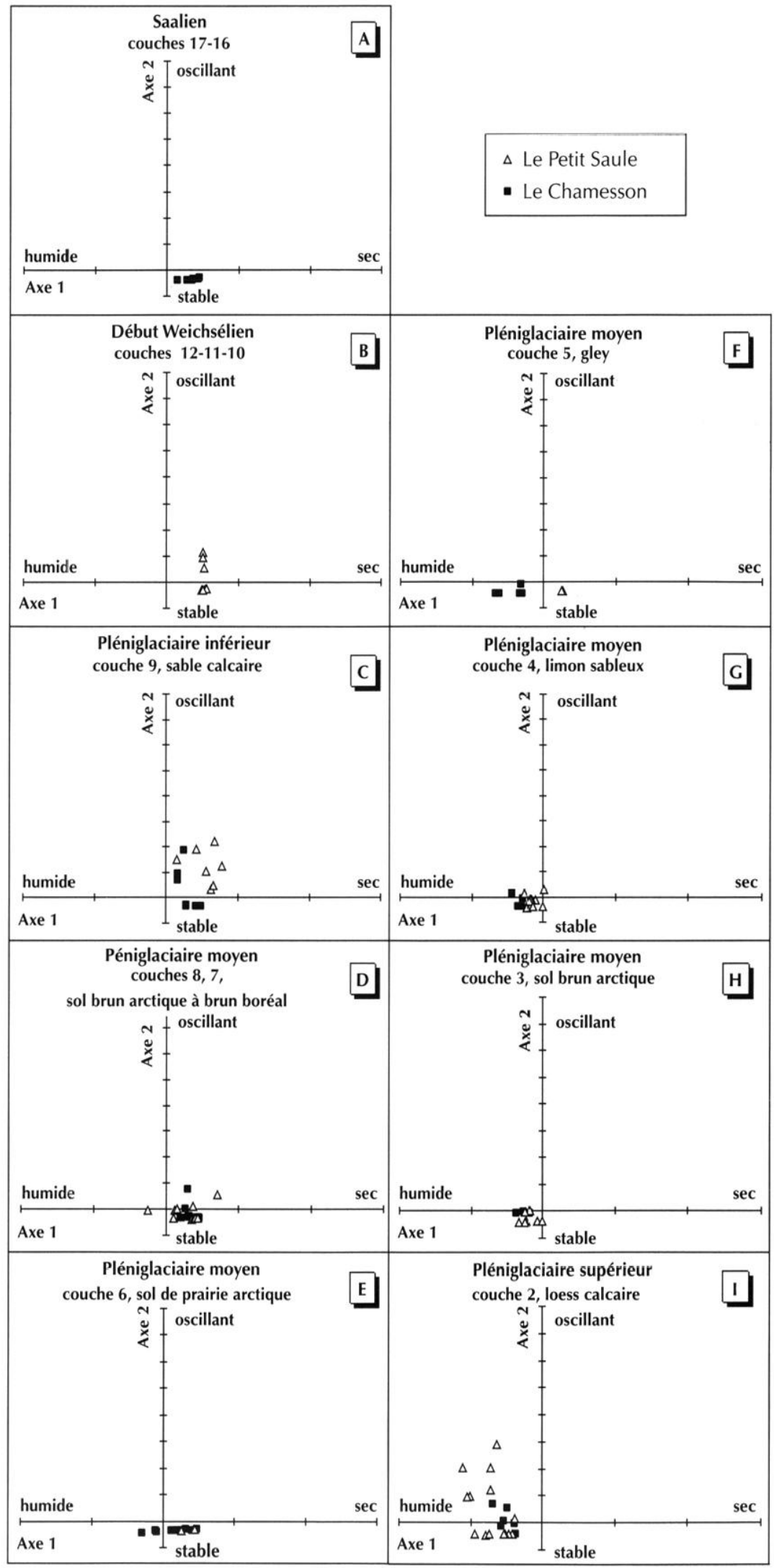

Fig. 29 - Répartition des prélèvements dans le premier plan factoriel.

Les triangles correspondent à la série du Petit Saule, les carrés à celle du Chamesson. 


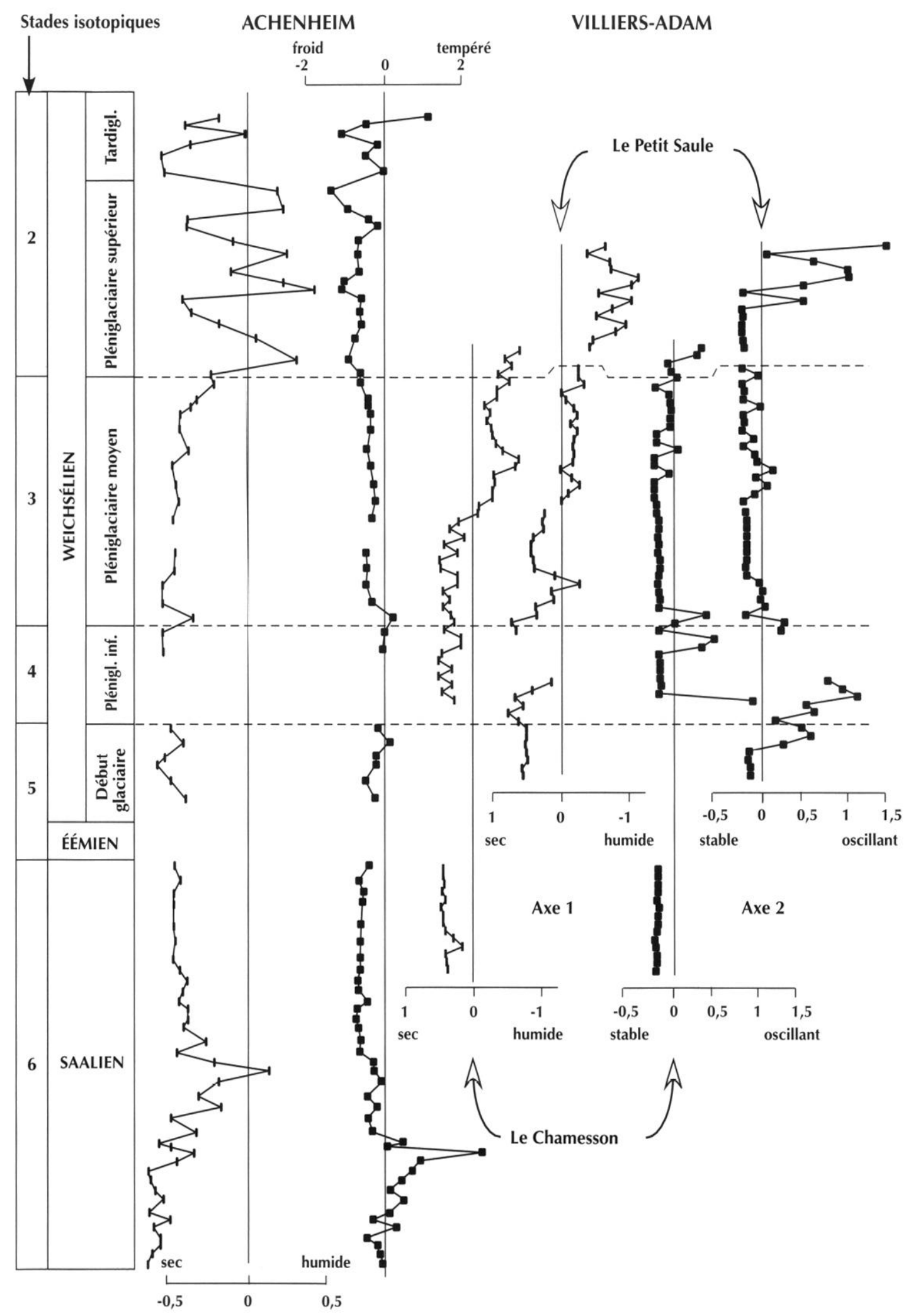

Fig. 30 - Courbes des variations relatives des paramètres climatiques exprimées par les malacofaunes des sites d'Achenheim et de Villiers-Adam. Les associations d'Achenheim sont réparties sur $28 \mathrm{~m}$ d'épaisseur, l'équidistance entre les échantillons de Villiers-Adam est de $10 \mathrm{~cm}$. Les courbes de l'axe 1 du Chamesson et du Petit Saule ont été inversées pour faciliter la comparaison avec les données alsaciennes. 
d'associations glaciaires définies par J.J. Puisségur (1976) pour nos régions, celui auquel se rapportent les faunes de Villiers-Adam " associations à Pupilla " correspond au faciès le plus sec. Les malacofaunes de phase plus froide et plus humide sont dites " associations à Columella ». J.-J. Puisségur (1976) a signalé que, dans le Bassin parisien, l'espèce Columella columella est plus rare que dans l'Est, et a invoqué le caractère moins continental du climat pour expliquer cette distribution. L'influence atlantique, en réduisant les écarts de température, limite l'extension de plusieurs taxons et provoque l'appauvrissement spécifique des associations. Cependant C. columella a été recensée dans des séries très occidentales en Angleterre (Kerney, 1971 ; Preece, 1990) et en Bretagne (Rousseau et al., 1990). Sa présence est alors interprétée comme un signe de conditions environnementales particulières propres au gisement. Ainsi son absence à Villiers-Adam peut être considérée comme significative de conditions moins humides dans le cadre de comparaisons inter-sites.

L'axe 2 n'est bien caractérisé que sur son pôle positif, par certains mollusques sténothermes. Si les eurythermes se retrouvent très logiquement dans la partie négative de cet axe, il n'en demeure pas moins qu'aucune espèce ni association ne présente de contribution relative élevée à l'inertie de l'axe. Le poids des taxons et des assemblages de phase froide n'est pas pris en compte par l'analyse factorielle. Ainsi les variations des courbes de Villiers-Adam bâties d'après les données de l'axe 2 doivent être comprises comme exprimant la différence entre des phases durant lesquelles les conditions de température oscillent et des phases sans changement des conditions de température, plutôt que comme des contrastes absolus de réchauffement-refroidissement.

Sur les courbes d'Achenheim et de Villiers-Adam, la phase finale du stade 6 est un épisode de sècheresse et de températures stables. À Achenheim, les malacofaunes sont significatives d'un refroidissement marqué (Rousseau, Puisségur, 1990). Cette interprétation peut également être proposée pour les associations de la coupe saalienne de Villiers-Adam qui ne comportent aucune espèce sténotherme et apparaissent caractéristiques d'une phase pléniglaciaire.

Au cours du Weichsélien à Villiers-Adam, les courbes de l'axe 1 indiquent une augmentation régulière de l'humidité pendant le Pléniglaciaire moyen, à partir des sols de prairie arctique (niveaux 7 et 6 ). Dans les dépôts correspondant au Pléniglaciaire supérieur, l'humidité reste importante, mais les fluctuations de ce paramètre sont plus rapides et contrastées. Les courbes de l'axe 2 montrent des phases d'oscillation des températures à la transition Début Glaciaire/Pléniglaciaire inférieur, puis Pléniglaciaire inférieur/Pléniglaciaire moyen, ainsi que durant le Pléniglaciaire supérieur.

La stabilité des températures au cours du Pléniglaciaire moyen est bien traduite par les successions malacologiques des deux séquences. En revanche, cette phase apparaît plus sèche sur le site d'Achenheim. À Villiers-Adam, l'augmentation de l'humidité, qui repose sur la distribution de l'espèce hygrophile $S$. oblonga et se trouve corroborée par les données palynologiques (voir p. 32), a certainement une valeur locale. Cependant, les données sur le Bassin parisien sont encore insuffisantes pour déterminer si ce phénomène a une extension régionale.

Enfin, le système oscillant complexe, qui est une caractéristique climatique majeure du stade 2 mise en évidence à Achenheim (Rousseau, 1987, 1989a, 1989b ; Rousseau, Puisségur, 1990), est également bien perçu par les successions malacologiques de Villiers-Adam.

\section{Conclusion}

Villiers-Adam est le premier site du Bassin parisien où il a été possible d'étudier des successions de malacofaunes lœssiques en continu sur une phase longue du Pléistocène. L'analyse de la structure des populations de mollusques a montré que globalement le paysage est très ouvert, de type steppique, et change peu. Ceci a été confirmé par les analyses palynologiques concernant une partie du Pléniglaciaire moyen du Weichsélien (voir p. 47) (fig. 31). Au Saalien, le milieu est sec et les faunes témoignent, par leur extrême monotonie, de la permanence des conditions environnementales et, par leur pauvreté spécifique, de la rigueur climatique. Au début du Weichsélien, pendant une phase couvrant le complexe initial de paléosols et la base des dépôts du Pléniglaciaire inférieur, les associations malacologiques sont plus riches en espèces, même si l'occurence des nouveaux taxons reste très sporadique, témoignant ainsi de températures plus variables. La deuxième partie des niveaux du Pléniglaciaire moyen est marquée par l'évolution régulière des conditions locales vers une nette humidification et des températures monotones. Le Pléni- 


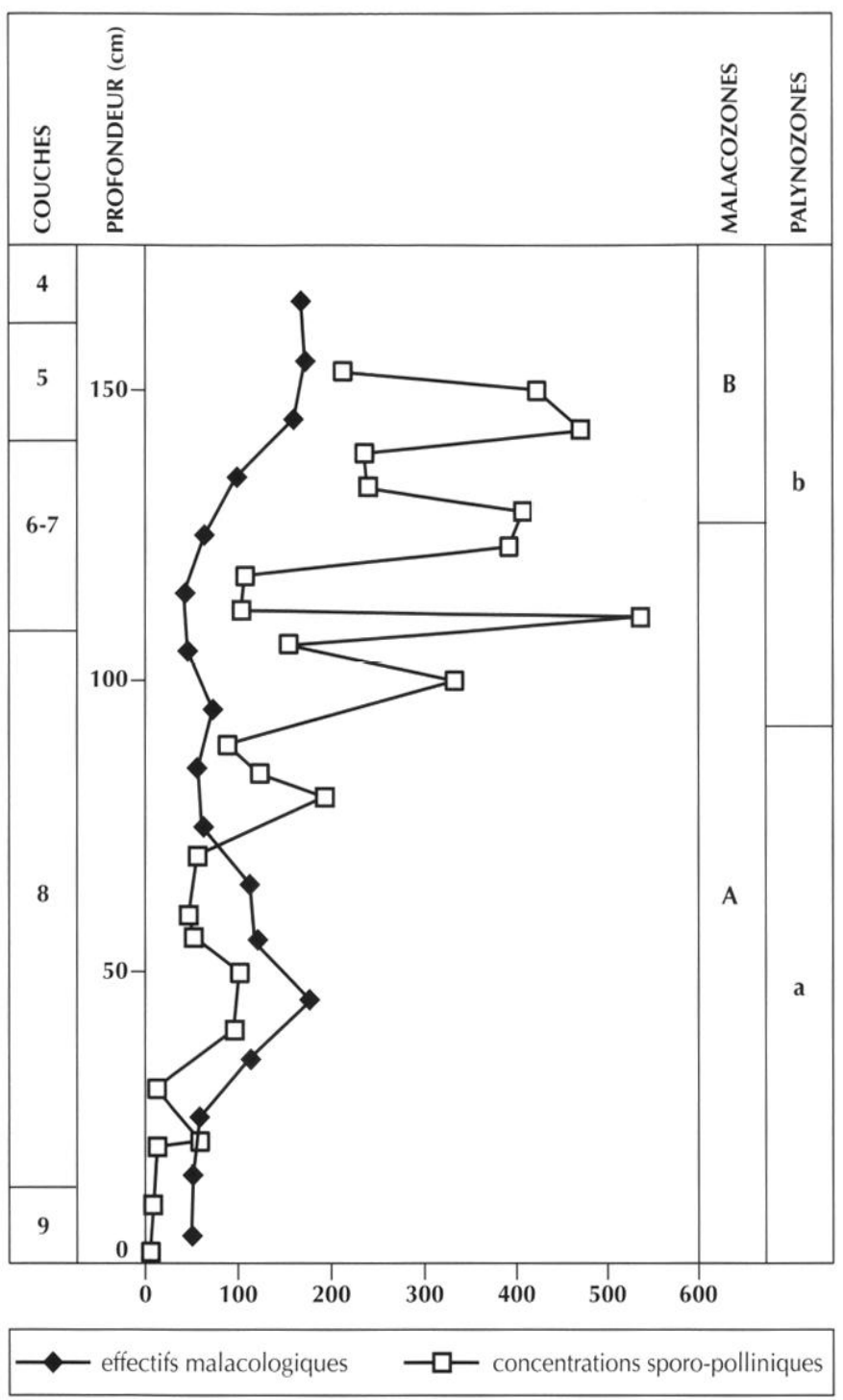

Fig. 31 - Comparaison des données palynologiques et malacologiques du Pléniglaciaire moyen du Weichsélien sur la coupe Talus locus 1-est du Petil Saule.

glaciaire supérieur, en revanche, est caractérisé par un système climatique oscillant.

La comparaison des successions de Villiers-Adam et d'Achenheim en Alsace fait ressortir la bonne convergence des données malacologiques sur l'évolution des paramètres climatiques. Les températures sont cependant moins bien enregistrées par les malacofaunes de Villiers-Adam qui rendent compte des phases d'oscillation plutôt que des contrastes. Ceci est peut-être lié à la pauvreté spécifique des associations, caractéristique de la zone lœessique occidentale. Plus vers l'est, les réservoirs biologiques que sont les zones montagneuses fournissent des faunes beaucoup plus diversifiées, même en période glaciaire, qui permettent d'enregistrer plus finement toutes les composantes environnementales et climatiques ainsi que leurs variations. Malgré cette réserve, la succession de Villiers-Adam constitue par son ampleur un référentiel important pour le Bassin parisien et ouvre des possibilités de comparaison interrégionale.

\section{COMPARAISON DES DONNÉES PALYNOLOGIQUES ET MALACOLOGIQUES}

La comparaison des données palynologiques et malacologiques ne peut s'exercer que pour une partie du Pléniglaciaire moyen du Weichsélien, la coupe Talus locus 1-est du Petit Saule étant la seule à avoir livré des cortèges polliniques interprétables.

Les courbes des effectifs malacologiques et des concentrations absolues de grains de pollens (fig. 31) indiquent une mauvaise conservation à la base de la séquence (sommet de la couche 9) qui tend à s'améliorer vers le sommet. Dans le détail, l'échantillonnage palynologique beaucoup plus serré enregistre des variations que l'espacement régulier des prélèvements malacologiques (tous les $10 \mathrm{~cm}$ ) ne permet pas d'observer.

La distinction entre les deux palynozones (fig. 31) est basée sur une diversification des herbacées qui, conjuguée à l'augmentation des concentrations, révèle un couvert végétal plus dense au cours de la zone pollinique b. De plus, cette zone est caractérisée par deux épisodes durant lesquels des herbacées de milieux humides se développent. Ces phases rendent compte d'une humidité locale plutôt que climatique et sont identifiées respectivement dans les niveaux 7 (sol brun arctique) et 5 (gley) (voir p. 32). Les malacozones A et B (fig. 31) sont différenciées par la distribution de l'espèce hygrophile $S$. oblonga. Son développement dans la zone $\mathrm{B}$, à partir du niveau 7 , témoigne d'une augmentation de l'humidité locale. Elle présente un pic plus important dans la couche 5 (voir p. 32). Par ailleurs, il est intéressant de mettre en parallèle la densification du couvert végétal avec l'augmentation des effectifs malacologiques, les populations de mollusques étant très dépendantes de la végétation pour leur habitat et leur nourriture.

Les deux enregistreurs s'accordent sur la perception d'une humidité croissante à partir de la couche 7 et qui devient particulièrement accusée dans le gley. 
Du point de vue environnemental, les cortèges polliniques et malacologiques soulignent l'ouverture et l'homogénéité du milieu. Toutefois, l'analyse palynologique définit le paysage comme une toundra et l'étude malacologique comme une steppe lœssique. Cette apparente divergence s'explique par l'ordre de grandeur de l'échelle d'observation des deux disciplines qui est exprimée par une terminologie différente.

Les malacocénoses rendent compte des conditions environnementales au niveau du sol. Les associations dites de “steppe lossique " (Lozek, 1990, 2001) indiquent des milieux très ouverts (grands espaces herbacés) et relativement secs. L'analyse des cortèges polliniques est en accord avec l'interprétation malacologique, mais permet de préciser les composants de la couverture végétale régionale. Dans le cas de VilliersAdam, la présence d'arbustes nains parmi les graminées et les cypéracées est identifiée. En biogéographie, cette association de végétaux correspond à la toundra (Ozenda, 1994). Sur les spectres de Villiers-Adam, la présence de cette végétation arbustive est cependant sporadique et n'a donc d'incidence ni sur la nature du sol ni sur la composition des peuplements malacologiques. Ces résultats illustrent l'intérêt d'une approche pluridisciplinaire qui permet des reconstitutions paléoenvironnementales d'une plus grande précision.

\section{ISOTOPIE ORGANIQUE}

L'approche isotopique sur matière organique $\left(\partial^{13} \mathrm{C}_{\mathrm{org}}\right)$ a été utilisée pour la séquence de Chamesson. Le $\partial^{13} \mathrm{C}_{\text {org }}$ présente des valeurs toujours inférieures à - $23 \%$. Villiers-Adam, comme d'autres séquences lœssiques du nord-ouest de l'Europe (Hatté et al., 1998) et à l'inverse des lœess chinois (Wang et al., 1997; Lin et al., 1991), n'a connu qu'une végétation de type $\mathrm{C}_{3}$, tout au long des derniers 300000 ans (Hatté, 2000). Dans le cas d'un développement de végétation $\mathrm{C}_{3}$ dans un environnement non stressant (par des températures trop froides par exemple), les variations du $\partial^{13} \mathrm{C}$ peuvent être interprétées comme une réponse du système végétal aux fluctuations de la disponibilité en eau (Hatté et al., 1999) : les valeurs les plus négatives correspondant aux environnements les plus humides et les $\partial^{13} \mathrm{C}_{\text {org }}$ proches de - $23 \%$ à des conditions de forte aridité. De plus, dans un environnement de dépôt particulier ne

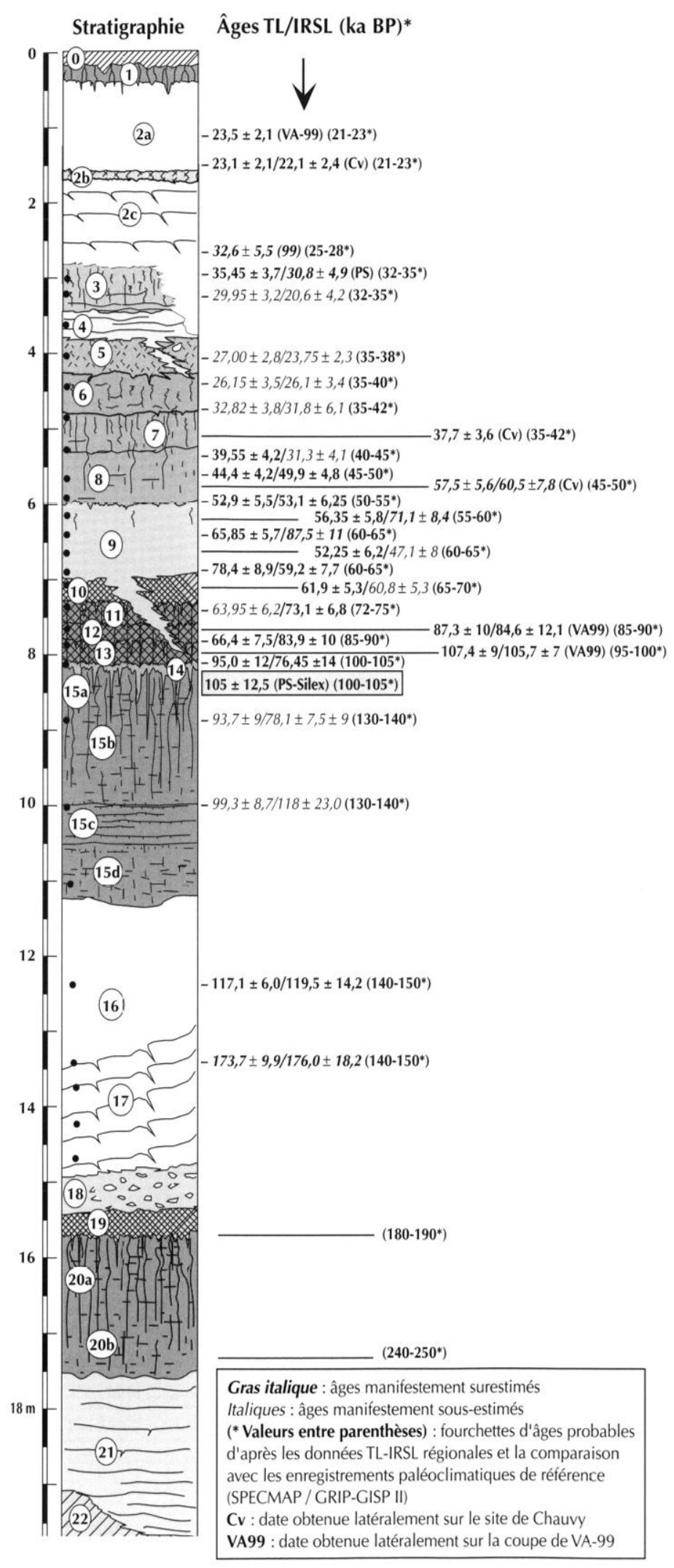

Fig. 32 - Villiers-Adam : séquence pédostratigraphique synthétique. et datations TL-IRSL (dessin P. Antoine). 
dégradant pas le signal isotopique (forte accumulation, faible rhizosphère et faible activité bactérienne), c'està-dire dans les phases glaciaires de dépôt lœssique, il est possible de quantifier cette disponibilité en eau et d'en tirer une estimation de précipitations (Hatté et al., 2001).

L'interprétation complète du signal isotopique (Hatté, 2000) a permis de proposer un environnement de dépôt très froid pour le Glaciaire saalien (environnement stressant biaisant le signal isotopique, phénomène corroboré par les très faibles teneurs en carbone organique), une glaciation particulièrement aride $\left(\partial^{13} \mathrm{C}\right.$ élevés) pour le Glaciaire saalien supérieur et une succession de phases sèches et plus humides pour le Weichsélien. Cette interprétation est incorporée dans la section qui suit immédiatement.

\section{ÉVOLUTION PÉDOSÉDIMENTAIRE, INTER- PRÉTATION CHRONOSTRATIGRAPHIQUE ET PALÉOENVIRONNEMENTALE}

Sur les bases de l'approche morphostratigraphique et pédostratigraphique précédemment exposée, la mise en évidence de l'évolution des environnements depuis la fin du Pléistocène moyen se fonde sur la synthèse des données sédimentologiques analytiques analytiques (fig. 13 et 14), les données bioclimatiques (mollusques et pollens), isotopiques $\left(\partial^{13} \mathrm{C}_{\mathrm{org}}\right)$, l'analyse micromorphologique et les données géochronologiques TL-IRSL (fig. 32).

Parallèlement, l'interprétation chronostratigraphique se base sur la corrélation entre la séquence d'événements

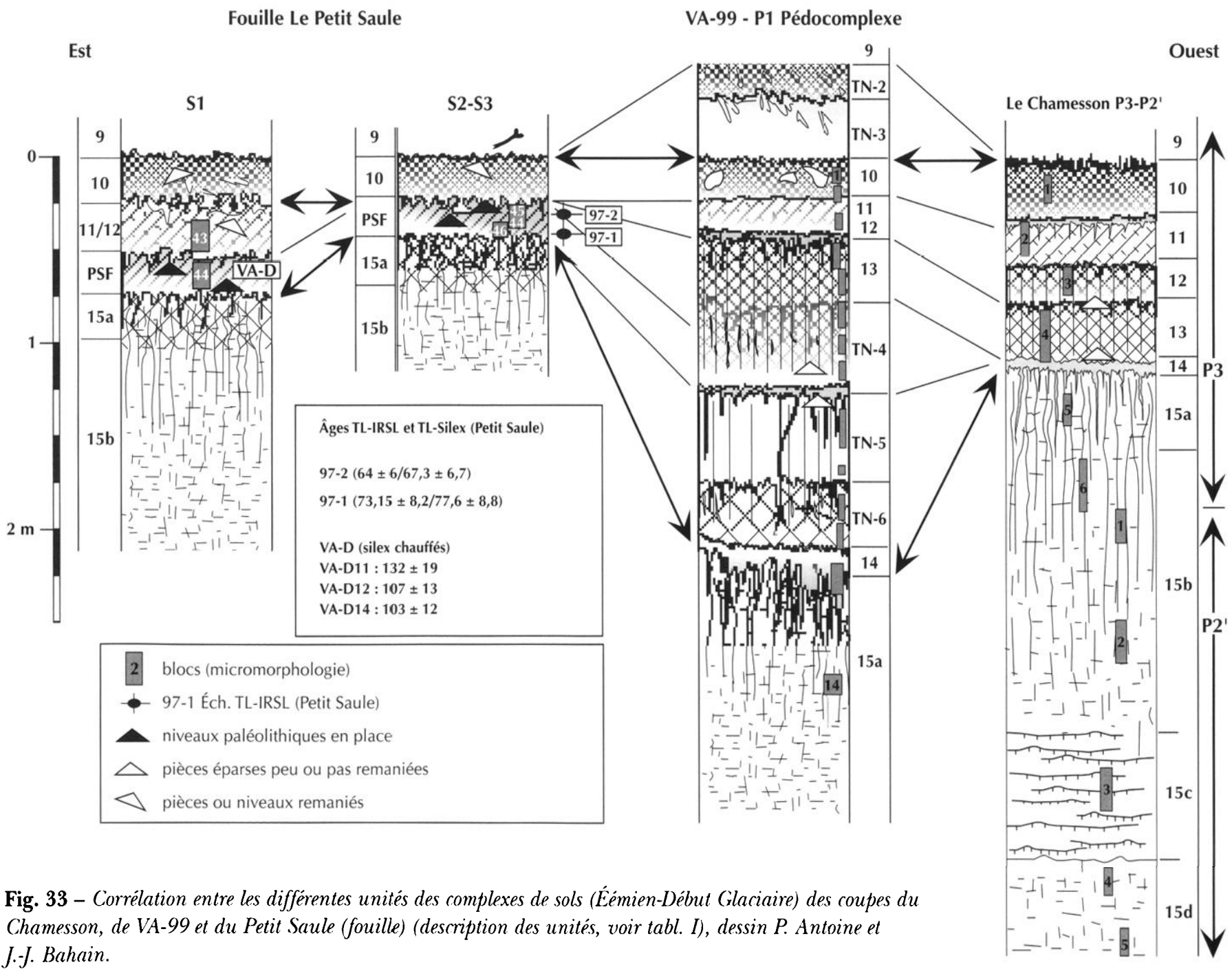
J.J. Bahain. 


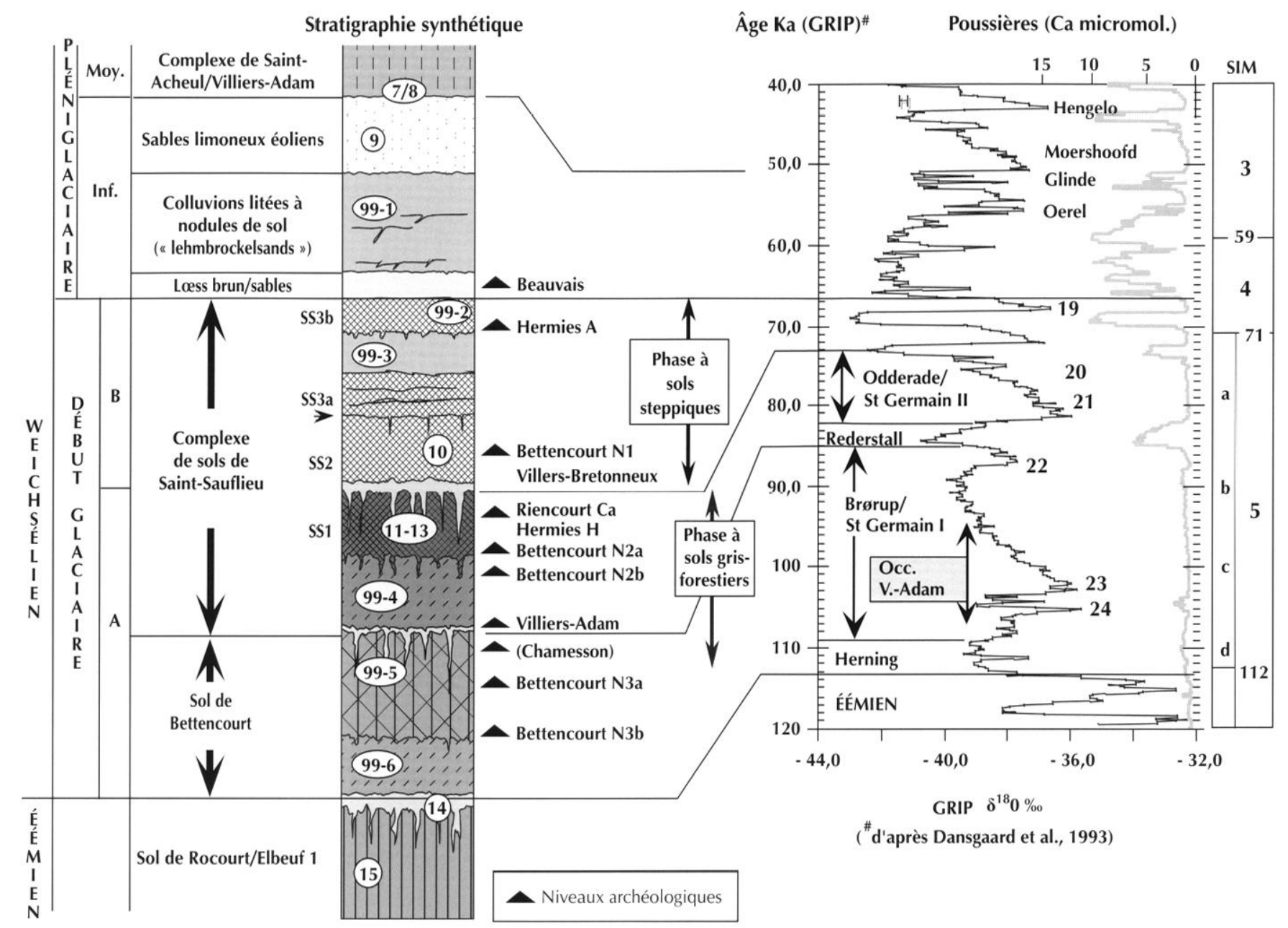

Fig. 34 - Localisation des occupations paléolithiques de Villiers-Adam et de quelques autres sites du Paléolithique moyen récent dans la séquence pédosédimentaire du Début (ilaciaire de la France septentrionale, corrélation avec les variations du $\delta^{\prime s} O$ de GRIP (dessin P. Antoine).

climato-sédimentaires décrite à Villiers-Adam (fig. 33) et la stratigraphie des formations loessiques de la France du Nord-Ouest (Antoine et al., 1998, 1999) (fig. 34), complétée par les données géochronologiques obtenues à Villiers-Adam (TL-IRSL).

L'interprétation chronostratigraphique et environnementale des séquences de Villiers-Adam est présentée ici en suivant les grandes coupures définies précédemment (voir p. 16-20), en partant de la séquence la plus ancienne vers la plus récente.

\section{SÉQUENCE 1 : GLACIAIRE SAALIEN - INTERGLACIAIRE INTRASAALIEN}

Comme pour les autres séquences de la formation de Villiers-Adam, la mise en place de la séquence 1 est précédée par une intense crise érosive qui débouche sur la formation d'une structure en vallon qui a servi de piège sédimentaire (fig. 11).

Cette érosion des sables tertiaires a totalement décapé les sols et les dépôts antérieurs dont il ne reste aucune trace à Villiers-Adam, excepté sous la forme d'un cailloutis à blocs de meulière présent à la base des séquences (unité 22).

La première unité sédimentaire qui colmate cette structure est représentée par des sables limoneux régulièrement lités (unité 21: sable fin $\approx 20 \%$ ) (fig. 13), dont la mise en place fait principalement appel au ruissellement et à une alimentation locale (remaniement des sables de Beauchamp). Il s'agit d'une séquence de comblement rapide en contexte de sol extrêmement découvert (absence de bioturbation et de stabilisation du 
versant). Néanmoins, l'absence de mollusques et de pollens dans cette unité ne permet pas de pousser plus loin la reconstitution des conditions environnementales contemporaines de la mise en place de ce dépôt.

La partie supérieure de cette unité est affectée par le développement d'un sol de type brun lessivé (unités 20a et 20b) nettement caractérisé par une forte augmentation du pourcentage d'argile (fig. 14), la présence d'argilanes brun-rouge et de nombreuses biogaleries. Ce sol présente une structure polyédrique à lamellaire, qui indique une phase de gel saisonnier profond ayant affecté l'ensemble du profil et constitue la première manifestation du retour de conditions froides de la base de la séquence 2, que soulignent par ailleurs les phénomènes d'hydromorphie marquant aussi le sommet de cet horizon.

Le contenu en carbone organique total, ainsi que les valeurs de susceptibilité magnétique (fig. 14) sont en revanche beaucoup plus faibles que dans les autres complexes de sols interglaciaires, en raison du caractère très drainant des matériaux de base (sables limoneux, contexte fortement oxydant, non carbonaté).

Enfin, les variations du pourcentage d'argile dans le complexe 20a-20b semblent traduire un sol complexe résultant de la superposition de deux horizons $B_{t}$ séparés par un faible épisode sédimentaire (fig. 14). Compte tenu de la position du complexe de sols 20a-20b dans la stratigraphie, cette bipartition pourrait traduire un bilan de la réponse aux deux améliorations climatiques principales du stade isotopique 7 (Martinson et al., 1987). Néanmoins, contrairement à d'autres séquences où ces deux horizons sont nettement séparés par un dépôt loessique sableux (Balescu, 1988; Antoine, 1990), à Villiers-Adam, les deux phases de pédogenèse sont très difficiles à distinguer en raison de la faible épaisseur de dépôt qui les sépare, comme dans la séquence de Cagny.

\section{SÉQUENCE 2 : GLACIAIRE SAALIEN SUPÉRIEUR - INTERGLACIAIRE ÉÉMIEN}

La première unité de la séquence 2 est constituée par un petit sol grisâtre, faiblement humifère, affecté par une forte hydromorphie terminale (unité 19). Il correspond à un faciès de sol steppique ultérieurement dégradé et partiellement érodé lors du début d'une nouvelle période glaciaire. Il peut être rapproché du «sol gris » de Mautort qui, dans la Somme, marque le début du Saalien supérieur (Antoine, 1990). Lui succède une phase d'érosion intense qui se traduit par la création d'un vallon dont la base est soulignée par un cailloutis remaniant des éléments tertiaires du plateau (blocs de meulière) (unité 18, fig. 8).

Dans cette structure créée lors d'une phase déjà avancée du Saalien supérieur se déposent des loess calcaires lités à microfentes de dessiccation (fig. $35, n^{\circ} 2$ ), passant vers le sommet à des loess calcaires homogènes $\left(\mathrm{CaCO}_{3}: 10-14 \%\right)$.

Cette succession traduit des conditions froides et arides nettement soulignées par le contenu malacologique de type faune à Pupilla et la faible diversité spécifique des cortèges malacologiques (voir p. 35). Les âges TL-IRSL obtenus pour ces dépôts confirment leur attribution au Saalien supérieur (stade 6) et renforcent leur corrélation avec les séquences des régions avoisinantes (Mautort et Plachy-Buyon dans la Somme: Antoine, 1990 ; Locht et al., 1995a).

Les valeurs du $\partial^{13} \mathrm{C}$ confirment une aridité importante du milieu qui atteint son maximum au sommet de l'unité de loess homogènes 16. Enfin il est intéressant de noter que la succession de ces faciès loessiques de la fin du Saalien présente de fortes analogies avec celle de la fin du Weichsélien (Antoine et al., 1999) et indique des conditions d'aridité maximales, comparables à celles du Pléniglaciaire supérieur (30-15 ka BP).

La partie supérieure de ces loess calcaires est ensuite affectée par le développement d'un sol brun lessivé complexe particulièrement développé $(2,5 \mathrm{~m})$, qui traduit une nouvelle amélioration climatique de rang interglaciaire (unités 15a à 15d) (fig. 8). Ce sol, corrélable avec le sol de Rocourt (Gullentops, 1954), se caractérise par un faciès pédologique très complexe (sol polyphasé), dont seule la phase initiale est attribuable à l'Interglaciaire éémien (5e). En effet, les traits pédologiques des horizons supérieurs (15b et 15a) correspondent essentiellement à des processus pédologiques et de gel profond postérieurs, en liaison avec les phases initiales du Début Glaciaire. L'âge TL-IRSL obtenu dans la partie inférieure de ce sol complexe (fig. 8 et 32), $121 \pm 13$ ka BP (éch. 97-22), se distingue par une certaine sous-estimation, comme c'est souvent le cas dans les pédocomplexes du dernier interglaciaire (Frechen, 1999). Il est possible que ce phénomène soit lié à l'impact des différentes phases de pédogenèse et de gel saisonnier postérieures (variation de la dose externe en 
liaison avec la migration des argiles et/ou des silts de lavage de gel-dégel).

D'une manière détaillée, cet horizon $\mathrm{B}_{1}$ se subdivise en plusieurs sous-horizons qui se caractérisent par une altération de plus en plus poussée, soulignée par une augmentation progressive des teneurs en argile de $15 \%$ à $30 \%$ (fig. 14).

L'horizon inférieur $15 \mathrm{~d}$ se distingue par une décarbonatation totale et une altération peu marquée (rares revêtements argileux sur les biopores), un pourcentage d'argile, de carbone organique et des valeurs de susceptibilité magnétique toujours très faibles. On remarque que les valeurs de $\partial^{13} \mathrm{C}$ y sont encore très hautes et correspondent toujours à celles du lœess saalien non altéré. L'horizon à doublets sus-jacent (15c) montre des caractéristiques similaires et se distingue uniquement par sa structuration à lamines centimétriques brunes ondulées. Il est proche des faciès à doublets décrits par J.-P. Lautridou (1985) à la base du sol Elbeuf 1, dans l'Eure, où il est interprété comme un proto $B_{t}$ qui s'individualise juste après les premières phases de décarbonatation dès la fin du Pléniglaciaire (accumulation préférentielle de particules argileuses et de limon fin sur les discontinuités héritées de la structuration gel-dégel).

L'altération, matérialisée par l'augmentation des taux d'argile et par des revêtements argileux en lame mince, n'apparaît vraiment qu'à partir de la base de l'horizon $B_{\text {, }}$ fortement structuré (15b).

La partie supérieure de l'horizon $\mathrm{B}_{t}$ se distingue en revanche par une forte augmentation du pourcentage d'argile qui atteint $30 \%$, par la densité des revêtements argileux visibles en lame mince et par l'apparition d'une structure polyédrique à lamellaire centimétrique de type gel saisonnier profond (Van Vliet-Lanoë, 1987, 1990; Huijzer, 1993). Cette tendance est couplée à une augmentation des valeurs de susceptibilité magnétique, du pourcentage de carbone organique (fig. 14). La diminution brutale du $\partial^{13} \mathrm{C}$ à partir de la base de l'horizon 15a traduit cette fois des conditions beaucoup plus humides dont le maximum est atteint dans la partie médiane de cet horizon et qui caractérisent les conditions interglaciaires.

Les données micromorphologiques montrent que la partie supérieure de 15a a enregistré une succession complexe de phénomènes pédologiques et périglaciaires qui débutent avec l'Interglaciaire éémien (revêtements argileux microlités jaune clair) et se poursuit lors des premières phases de la période glaciaire suivante lors du Début Glaciaire weichsélien (gel saisonnier profond illuviations argilo-humiques épaisses, à silto-humiques stratifiées, phase finale de gel saisonnier profond et formation de revêtements de silts lavés colmatant la porosité).

Au sein de la succession des unités $15 \mathrm{a}$ à $15 \mathrm{~d}$ (sol de Rocourt), et plus particulièrement dans les horizons supérieurs $15 \mathrm{a}$ et $15 \mathrm{~b}$, la pédogenèse initiale que l'on peut rapporter à l'Éémien (décarbonatation, altération et illuviations microlitées jaunes) est donc fortement masquée par l'impact des processus pédologiques et périglaciaires postérieurs, développés dans un contexte déjà très différent au Début Glaciaire weichsélien (Van-VlietLanoë, 1987 ; Haesaerts et al., 1999) (fig. 36, nos 3 et 4). L'horizon 15a, s'il s'individualise nettement du point de vue paléopédologique, et notamment sur la base des données micromorphologiques, ne représente cependant pas une unité pédosédimentaire distincte (pas de phase de dépôts différents des unités sousjacentes).

\section{SÉQUENCE 3 : GLACIAIRE WEICHSÉLIEN - INTERGLACIAIRE HOLOCÈNE}

\section{Complexe de sols du Début Glaciaire}

La séquence 3 débute dans tous les profils par une phase d'érosion très marquée qui tronque le sommet de l'horizon $B_{t}$ interglaciaire du sommet de la séquence 2 . Cette crise érosive traduit une première déstabilisation $\mathrm{du}$ paysage et le retour de conditions rigoureuses (ouverture du paysage, développement des processus de ruissellement), et provoque localement la formation de structures en vallon peu marquées (VA-99) (fig. 12 et fig. $35, \mathrm{n}^{\circ} 3$ ). Cependant elle ne modifie pas fondamentalement la morphologie du paysage acquise lors de la dernière phase de dépôt lœssique du Saalien (érosion pré-éémienne ?) (Haesaerts, Mestdagh, 2000).

Cette première crise climatique qui marque le début du Weichsélien dans toutes les séquences de l'Europe du Nord-Ouest (Haesaerts et al., 1999; Schirmer, 2000; Antoine et al., 1999, 2001) est corrélée avec le stade 5d des séquences $\partial^{18} \mathrm{O}$ des sondages océaniques et le stade de Herning de la chronostratigraphie de l'Europe du Nord-Ouest (112 \pm 4 ka BP) (Behre, 1989 ; Caspers, Freund, 2001) ainsi qu'avec l'oscillation Melisey I de 


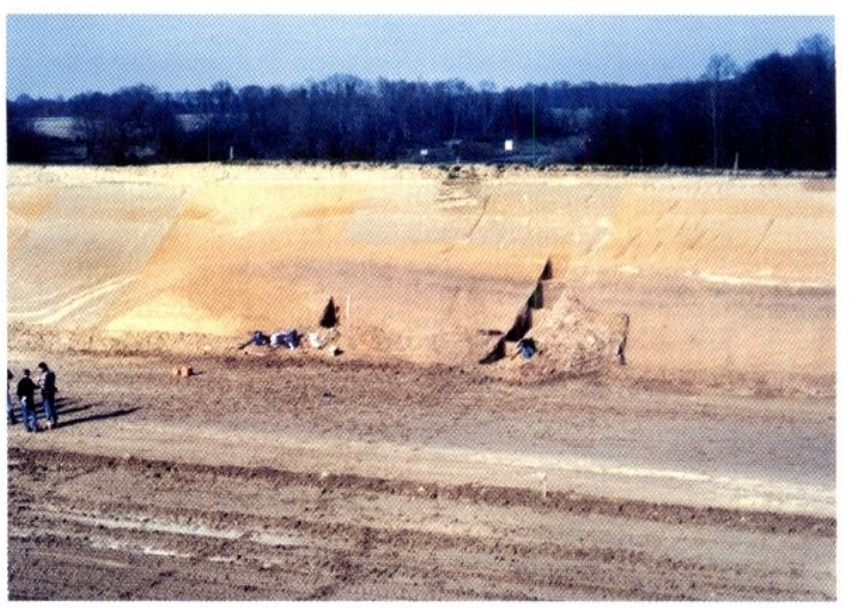

1

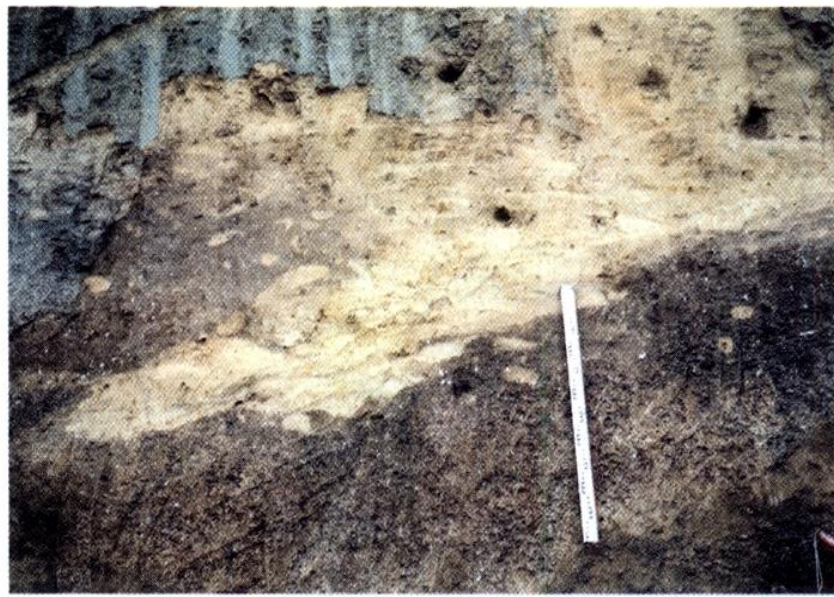

3

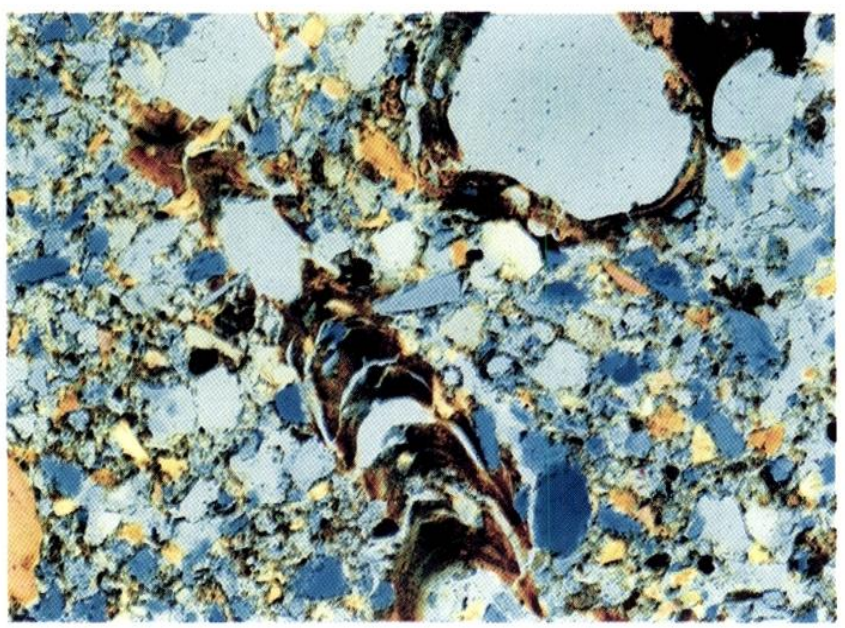

5

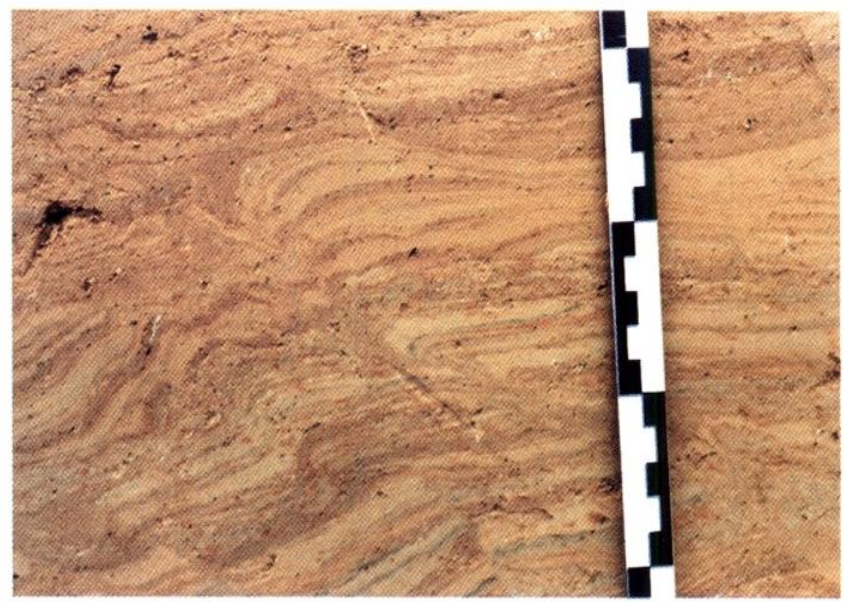

2

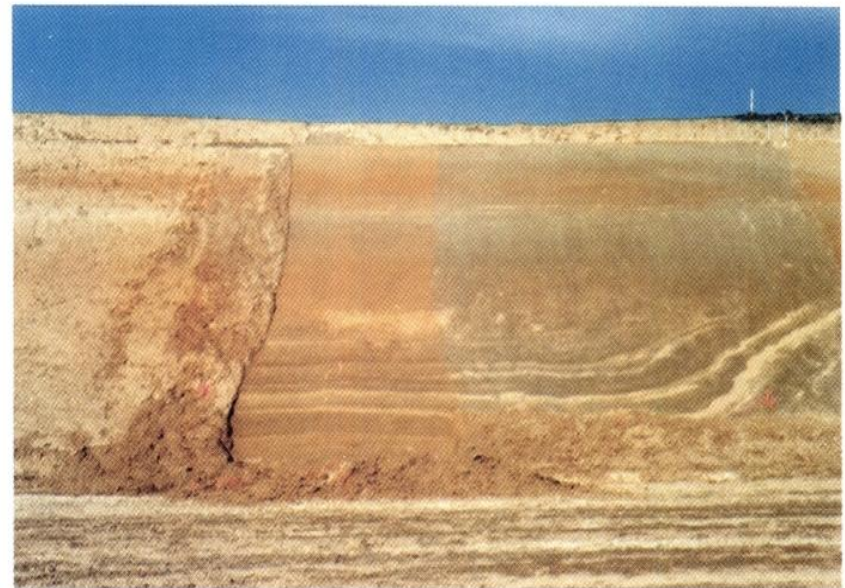

4

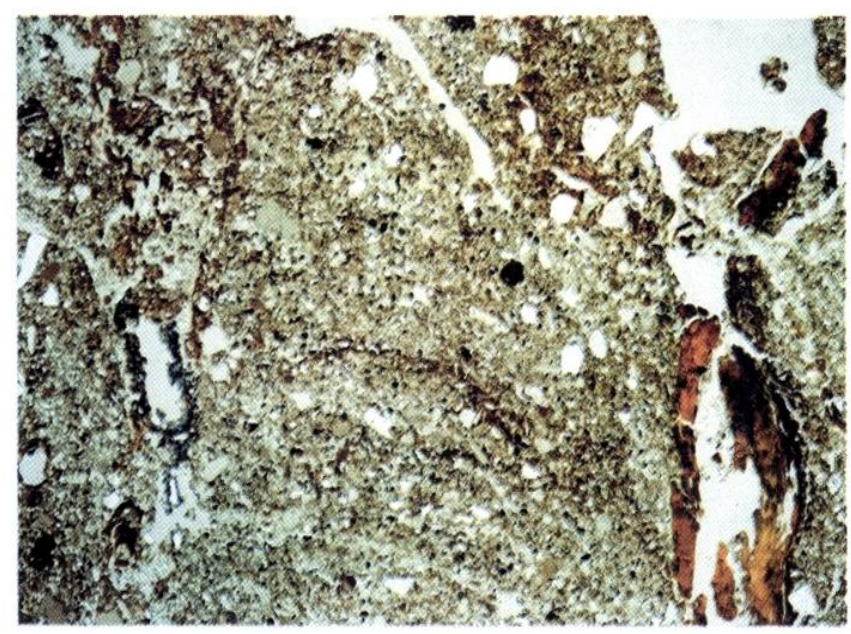

Fig. 35 - Détails géologiques et micromorphologiques des séquences pléistocènes de Villiers-Adam. LP, lumière polarisée ; LPA, lumière polarisée analysée (photos P. Antoine) :

1. Vue générale du profil Villiers-Adam 99.

2. Détail des loess calcaires lités à microfentes saaliens (unité 17).

3. Villiers-Adam, profil P4, structure d'érosion thermokarstique à remplissage sablo-limoneux (unité 9), affectant le sommet du complexe de sols humifères (unités 10 à 15 a).

4. Villiers-Adam 99, détail de la partie médiane de la coupe (voir fig. 12, P3, unités TN-1 à 2a).

5. Unitê 11 (Chamesson, fig. 8), argilanes humifères stratifiés associés à des biopores (taille de la vue: 1,2 mm), LPA.

6. Unité 12 base (Chamesson, fig. 8), colmatage d'une cavité biologique par silts stratifiés (logette d'insecte ?) (taille de la vue: $5 \mathrm{~mm}$ ), LP. 


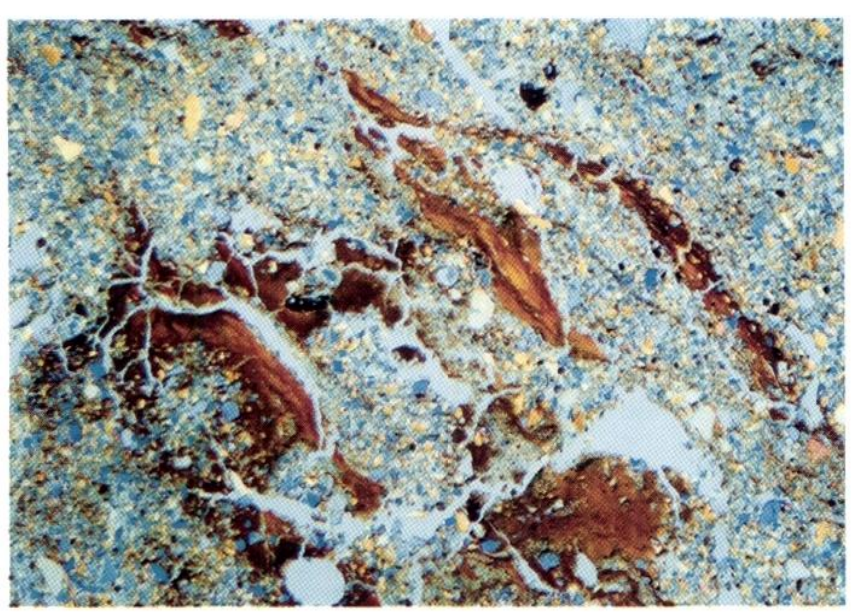

1

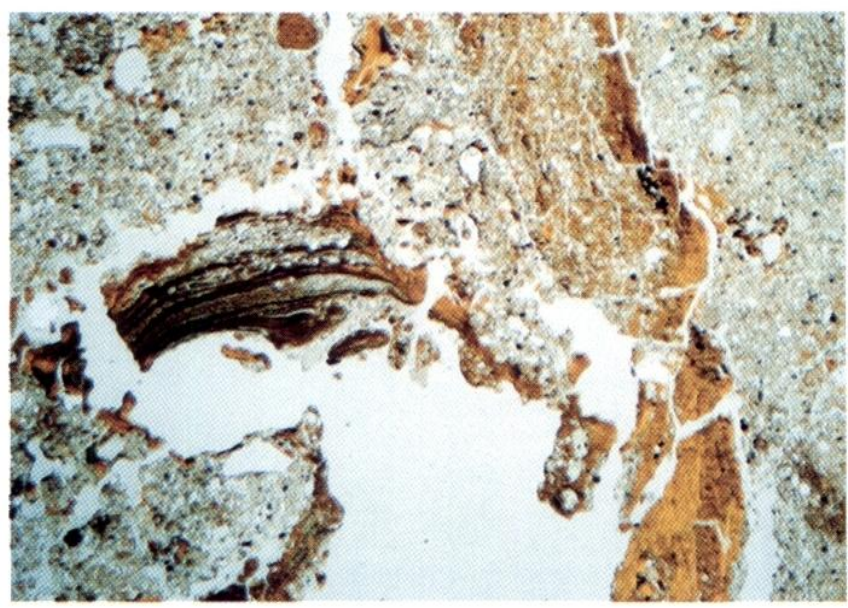

3

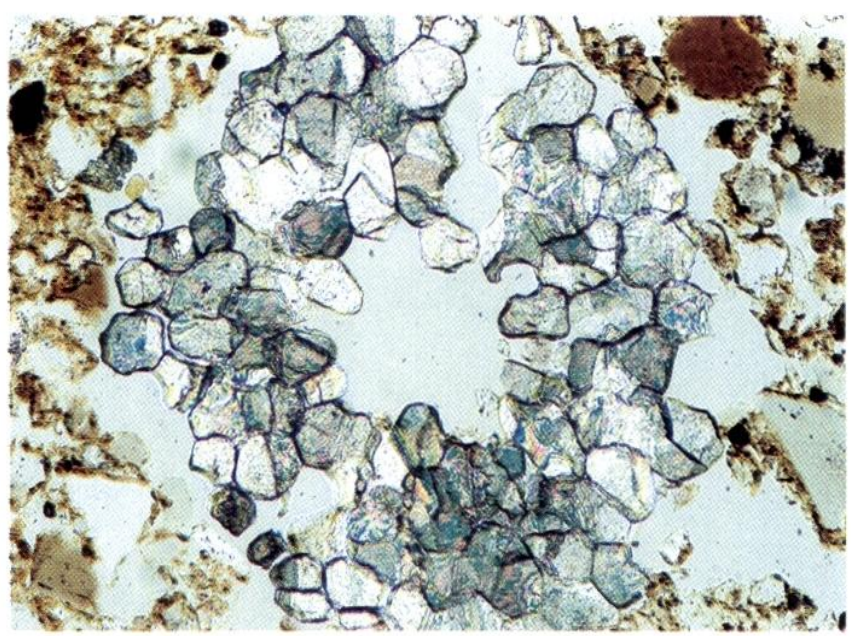

5

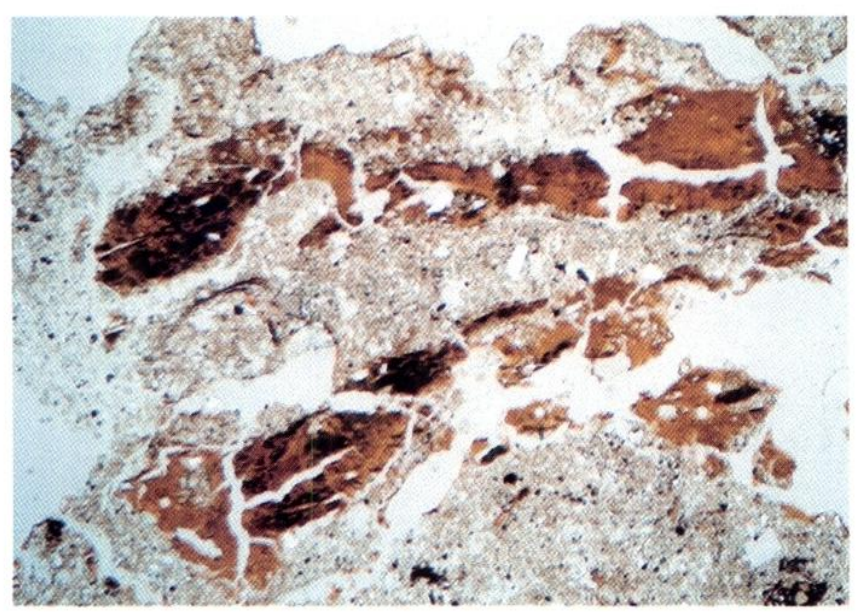

2

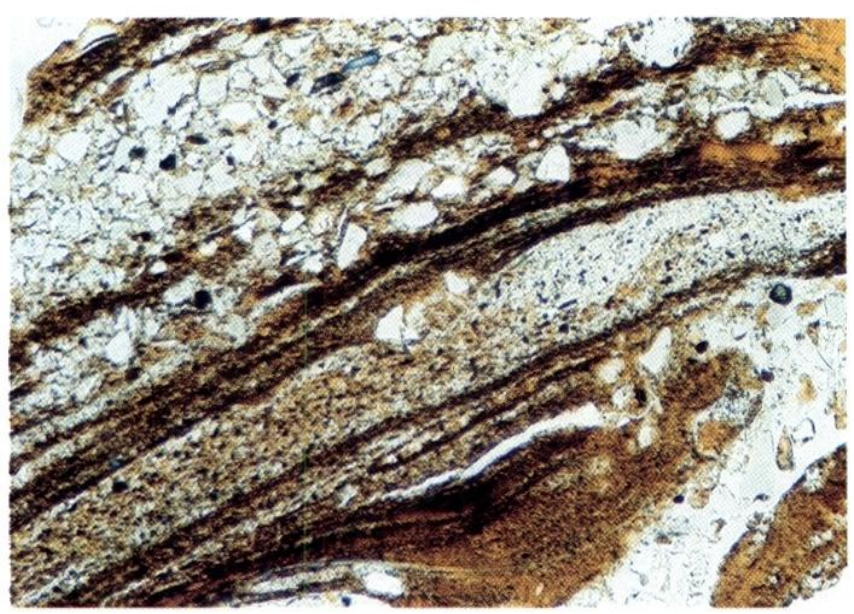

4

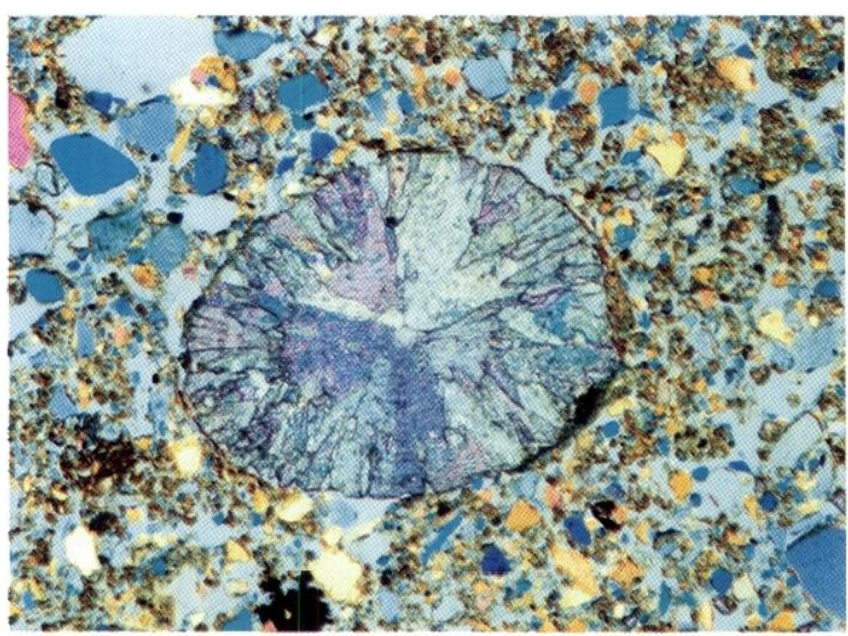

6

Fig. 36 - Détails micromorphologiques des séquences pléistocènes de Villiers-Adam. I.P, lumière polarisée ; LPA, lumière polarisée analysée (photos $P$. Antoine) :

1. Unité 13 sommet (Chamesson, fig. 8), revêtements argilo-humiques à silto-humiques stratifiés typiques du sol 13 (taille de la vue: $5 \mathrm{~mm}$ ), 1.PA.

2. Unité 13 base (Chamesson, fig. 8), revêtements argilo-humiques à silto-humiques stratifiés, fortement fragmentés par le gel et phase finale de colmatage silteux clair (taille de la zue: $5 \mathrm{~mm}$ ), $L P$.

3. Lnité 15 a (Chamesson, fig. 8), association ferriargilanes (jaune) et argilanes silto-humiques stratifiés au sommet du $B, 15 a$ (taille de la vue :

5 $(m m), L P$.

4. L'nité 15 a (Chamesson, fig. 8), détail des revêtements sillo-humiques stratifiés au sommet du B, 5 a (taille de la vne: 1,2 mm), L.P.

5. L'nité 8 base (Chamesson, fig. 8), détail d'un assemblage de cellules racinaires calcitées (hypocoating) (taille de la vue : 1,2 mm), I.P.

6. Lnile 7 (C.hamesson, fig. 8), biosphéroïde de calcite (sparite) ì structure filbro-radiée (vers de terre) (taille de la vue: $2,5 \mathrm{~mm}$ ), IIPA. 

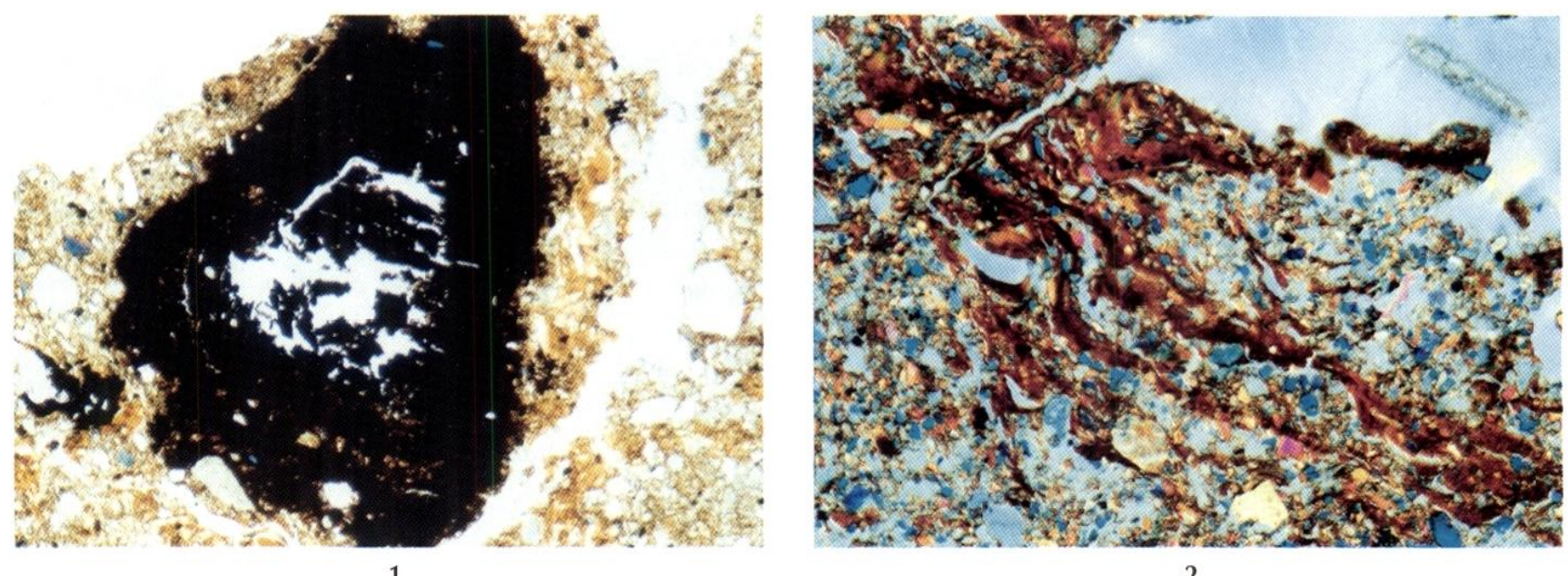

2
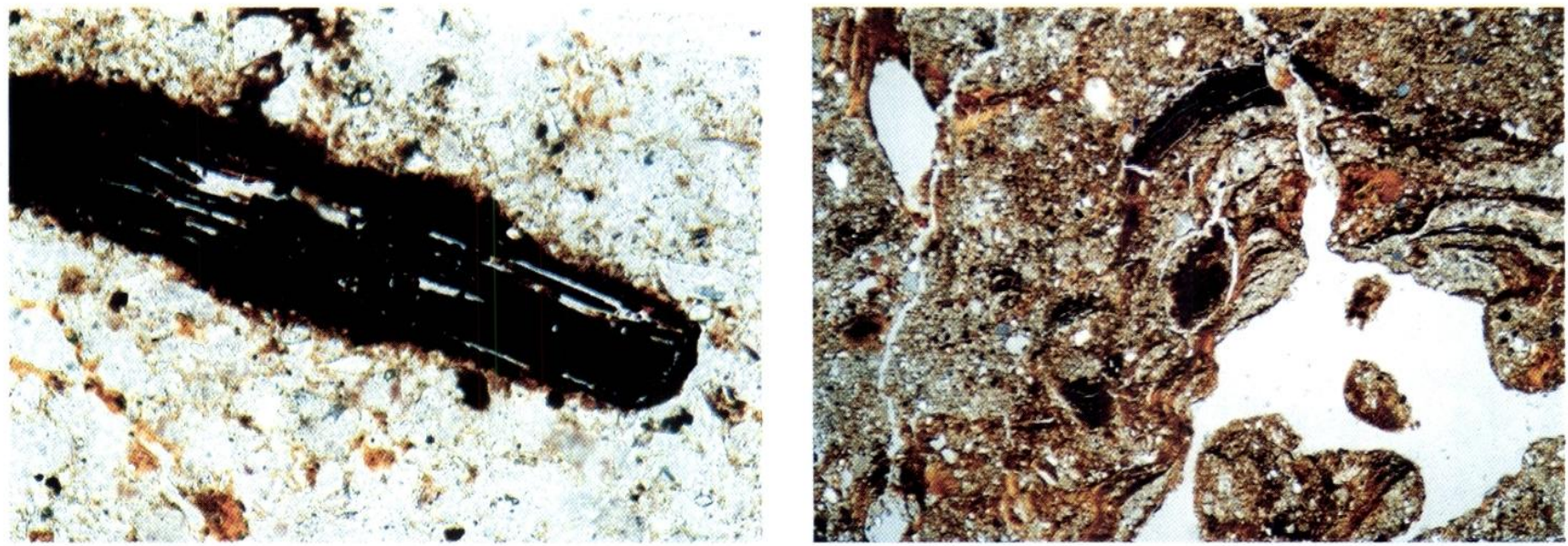

3

4

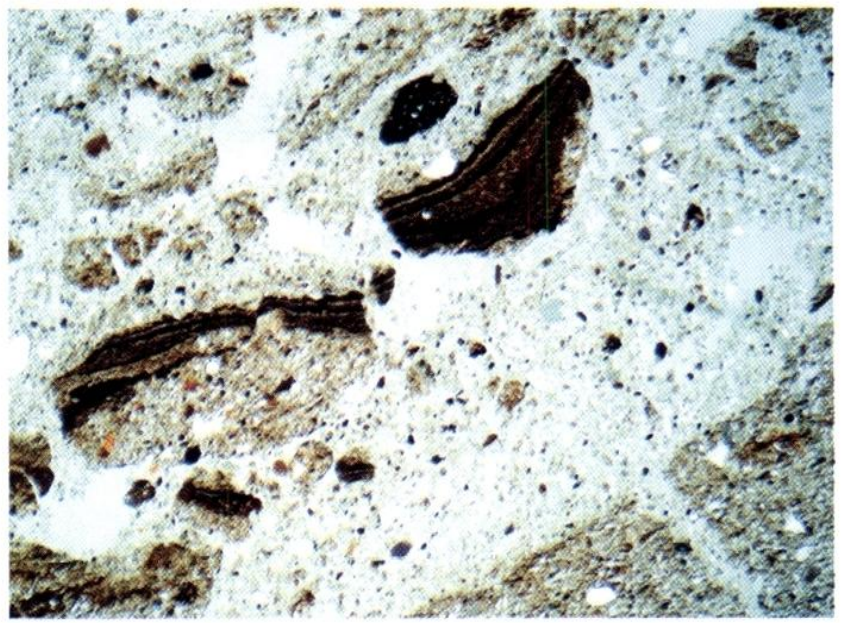

5

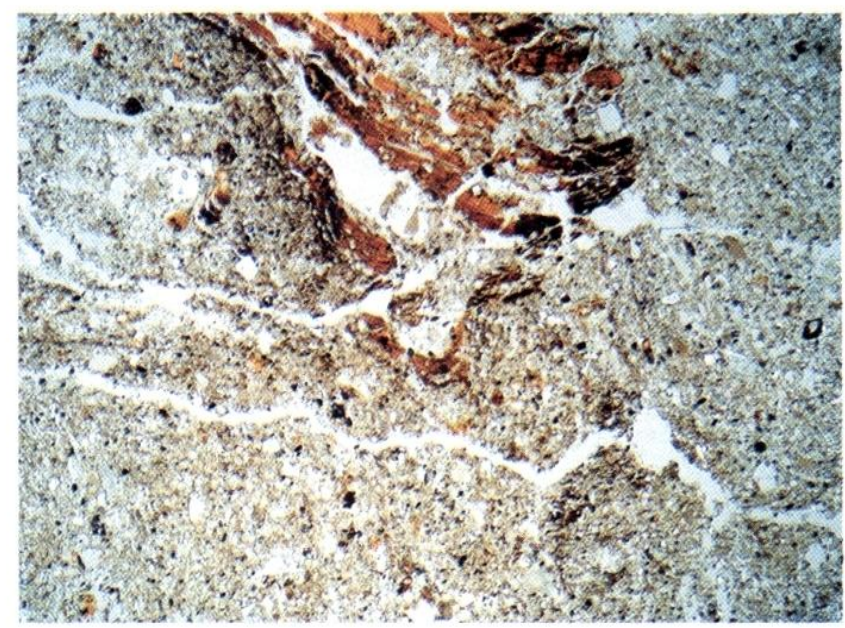

Fig. 37 - Détails micromorphologiques des séquences pléistocènes de Villiers-Adam. LP, lumière polarisée; LPA, lumière polarisée analysée (photos P. Antoine):

1. Unité TN-4 (VA-99, fig. 9), concrétion ferromanganique développée aulour d'un débris végétal humifié (taille de la vue : $2,5 \mathrm{~mm}$ ), LP.

2. Unité TN-5 (VA-99, fig. 9), revêtements argilo-humiques stratifiés à intercalations silteux iypiques du sol de l'unité TN-5 (taille de la vue :

2,5 mm), LPA.

3. Unité TN-6 (VA-99, fig. 9), débris végétal (ligneux) humifié typique de l'unité TN-6 (taille de la vue : 1,2 mm), LP.

4. Unité PSF (Petit Saule, fig. 35, lame 44), argilanes stratifiés siltohumiques et argilo-humiques en place avec phase de fracturation intense par le gel (taille de la vue : $2,5 \mathrm{~mm}$ ), LIP.

5. Unité PSF (Petit Saule, fig. 35, lame 44), remplissage silteux associé à des fragments de revêtements sillo-humiques stratifiés (croûtes de baltance) d'une grosse carvité biologique (taille de la vue: $5 \mathrm{~mm}$; diamètre de la cuvilé $\approx 1 \mathrm{~cm}$ ), $L P$.

6. Unité PSF (Petit Saule, fig. 35, lame 46), revêtements argilo-humiques à silto-humiques stratifiés fracturés par le gel (taille de la vue: $5 \mathrm{~mm}$ ), $L P$. 


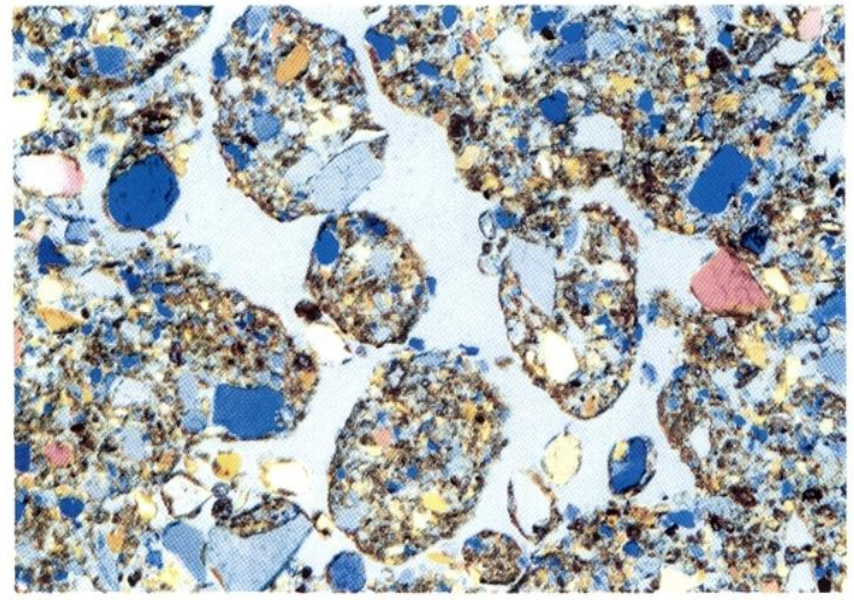

1

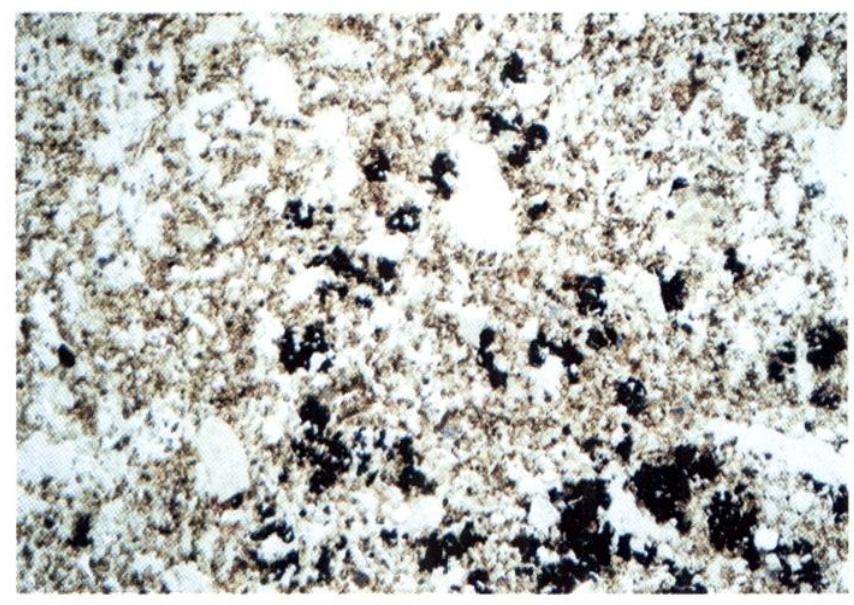

3

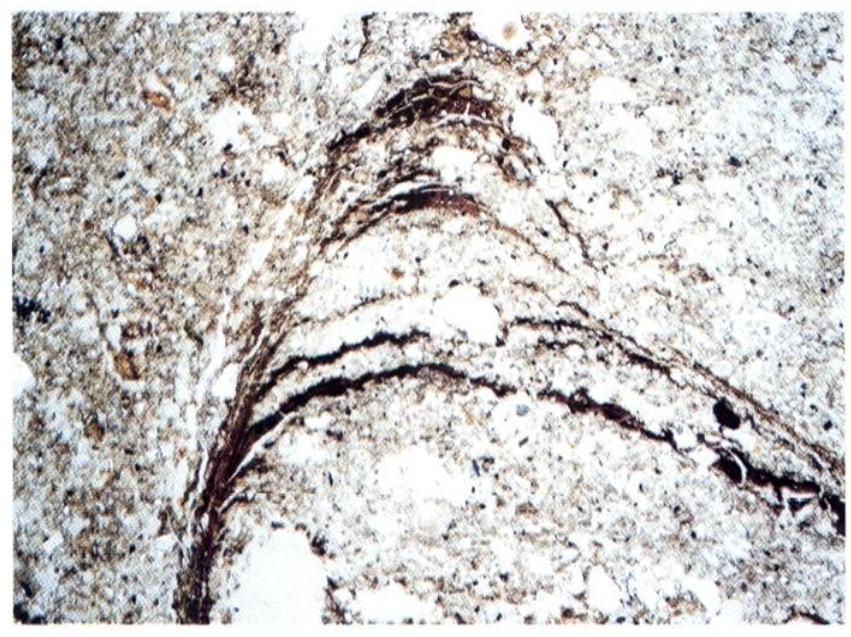

5

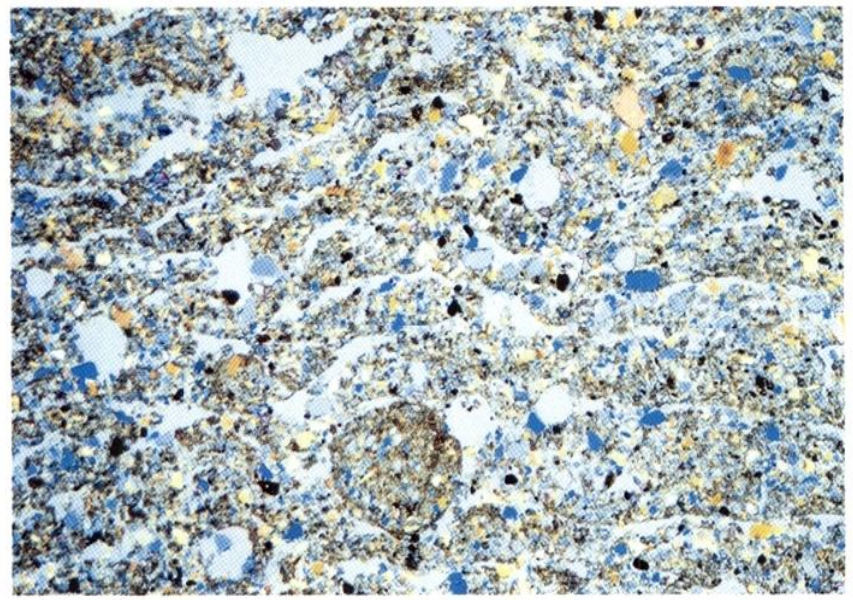

2

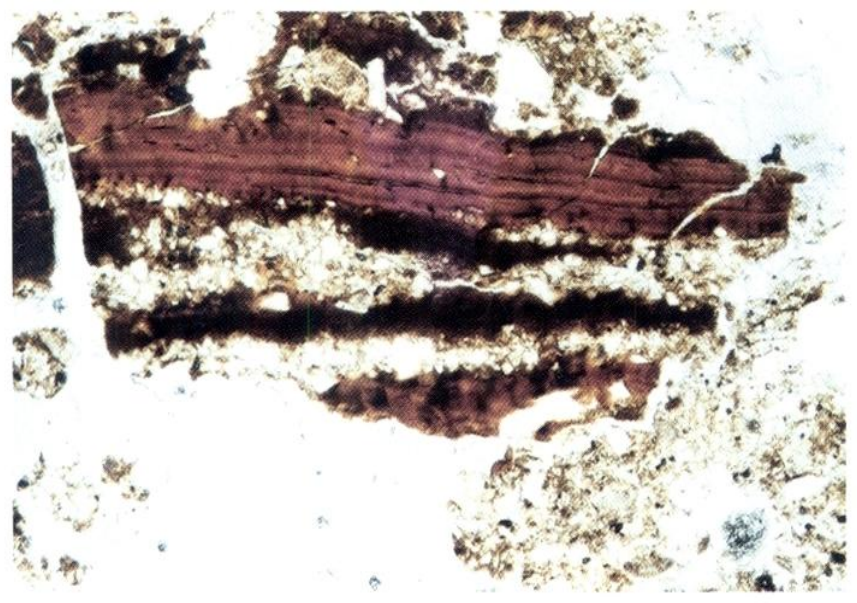

4

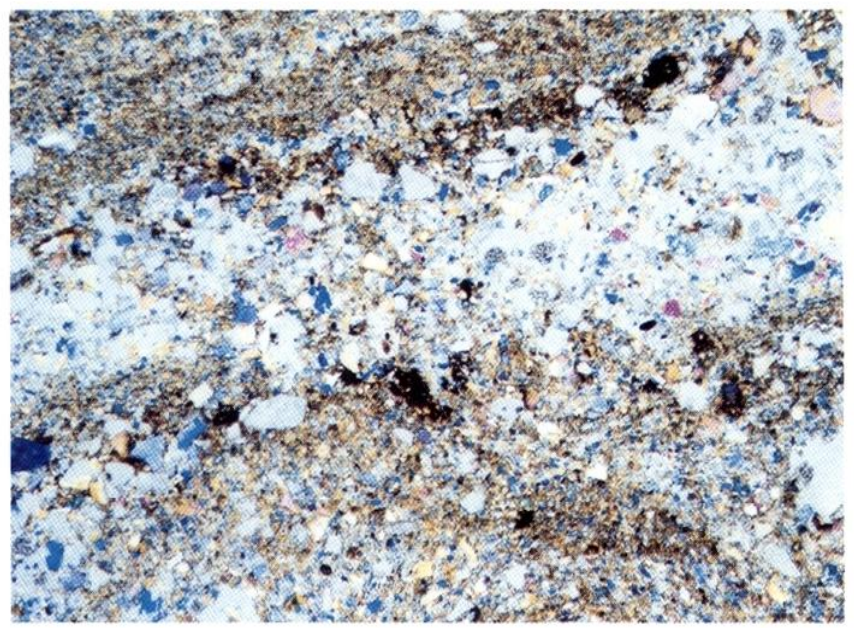

6

Fig. 38 - Détails micromorphologiques des séquences pléistocènes de Villiers-Adam. LP, lumière polarisée; LPA, lumière polarisée analysée (photos P. Antoine)

1. Unité 7 (Chamesson, fig. 8), pellets limono-sableux avec pellicule argileuse périphérique (déjections de vers ?) (taille de la vue: $2,5 \mathrm{~mm}$; diamètre $\approx 0,6 \mathrm{~mm}), L P A$.

2. Unité 7 (Chamesson, fig. 8), structure de lamellaire fine ondulée de type gel-dégel typique du gley de l'unité 5 , et déjection (taille de la vue :

$5 \mathrm{~mm}), L P A$.

3. Unité 10 (VA-99, fig. 9), structure du sol steppique de l'unité 10 avec abondantes précipitations ferromanganiques et matière organique éparses (micro-débris) (taille de la vue: $5 \mathrm{~mm}$ ), LP.

4. Unité 13 (VA-99, fig. 9), revêtement argilo-humique stratifié à intercalations silteuses (taille de la vue: 2,5 mm), LP.

5. Unité 13 base (VA-99, fig. 9), grande cavité biologique (insectes ?) avec colmatage silteux à silto-argileux humifère stratifié (taille de la vue : $5 \mathrm{~mm}), L P$.

6. Unité 13 base (VA-99, fig. 9), stratification originelle des colluvions limono-sableuses de l'unité 13, localement préservée de la bioturbation (taille de la vue: $5 \mathrm{~mm}$ ), LPA. 
La Grande Pile (Woillard, Mook, 1982). Cette période se traduit aussi dans les profils par une hydromorphie très nette (horizon blanchi 14 à glosses et nombreux nodules ferromanganiques), de très faibles valeurs de susceptibilité magnétique (fig. 8) et une chute des pourcentages en argile (fig. 14).

Au cours de cette phase, l'érosion du sol interglaciaire produit une première génération de colluvions limonoargileuses à nodules de sol remaniés, nombreux charbons de bois et microdébris végétaux humifiés (fig. $37, n^{\circ} 3$ ), dont la structure, à l'origine litée (ruissellement), est localement visible en lame mince (unité TN-6) (fig. 9). La présence du Chêne et du Noisetier au sein des charbons de bois récoltés parallèlement au Petit Saule lors de la fouille dans le niveau PSF, directement sus-jacent au B, 15a (Dietrich, com. pers. citée in Locht et al., 1997), confirme l'importance de la remobilisation de la partie supérieure du sol interglaciaire dans le processus de colluvionnement lors des premières phases du Début Glaciaire. Dans les profils de Villiers-Adam, l'absence de pollens dans le complexe de sol du Début Glaciaire ne permet pas, en revanche, de mettre en évidence un remaniement de pollens thermophiles dans les premières colluvions comme c'est le cas dans les séquences de référence de Saint-Sauflieu et de Bettencourt (Munaut in Antoine et al., 1994, 1998; Munaut in Locht dir., 2002).

$\mathrm{Au}$ sommet de cette première génération de colluvions, un sol se développe lors d'une première phase d'amélioration climatique et de stabilisation du paysage de type interstadiaire (unité TN-5). Cet horizon se distingue par une très forte compacité, une coloration brun sombre, une intense bioturbation (abondantes logettes de diapause/hibernation, et bioturbations à remplissage stratifié de type striotubules), une teneur en argile intermédiaire (maximum : $18 \%$ ), une teneur en carbone organique faible, des illuviations silto-argileuses humifères nettement stratifiées (fig. $37, \mathrm{n}^{\circ} 2$ ) et une importante phase finale de colmatage silteux (gel saisonnier profond). Cet horizon présente en fait des caractéristiques pédologiques et micromorphologiques intermédiaires entre celles d'un $B_{t}$ de sol brun lessivé et d'un $\mathrm{B}_{\text {th }}$ de type gris-forestier ou Greyzem (Van Vliet-Lanoë, 1990 ; Driessen, Dudal, 1991 ; Gerasimova et al., 1996 ; Shugalei, 1997).

Cet horizon qui présente des caractéristiques et une position stratigraphique proches de celles du " sol de
Bettencourt " défini dans la Somme (Antoine et al., 1995a ; Antoine, 2002), représente rraisemblablement la manifestation en milieu de versant du premier interstade forestier à forêt boréale du Début Glaciaire (interstade de Brørup/Saint-Germain I, corrélé avec le stade isotopique 5 c). Dans la coupe VA-99, un niveau d'industrie paléolithique diffus, comportant notamment une pointe Levallois, a été observé au sommet de cette unité à proximité de l'horizon glossique grisâtre qui marque sa limite supérieure.

Dans les profils où la première phase de colluvionnement n'est pas clairement enregistrée, comme au Chamesson, au Petit Saule ou encore à Chauvry (fig. 32), ces processus pédologiques et périglaciaires associés à l'oscillation d'Odderade/Saint-Germain II sont donc surimposés dans la partie supérieure tronquée du $B_{1}$ éémien. La partie supérieure de ce sol (unité TN-5) est ensuite affectée par une nouvelle dégradation climatique que l'on peut rapporter à l'oscillation froide de Rederstall ou Melisey II, vers 85 ka BP.

Cette dégradation se marque dans les coupes par un épisode de gel saisonnier profond avec le développement d'une structure polyédrique, une fragmentation des revêtements argileux et une hydromorphie très marquée, probablement exacerbée par les conditions topographiques locales en dépression (horizon à taches et langues grises déferrifiées, abondantes concrétions ferromanganiques).

La dégradation climatique se poursuit par la mise en place d'une nouvelle génération de colluvions limonoargileuses très compactes à nodules de sol et concrétions ferromanganiques remaniées (fig. $37, \mathrm{n}^{\circ} 1$ ), microcharbons, débris humifiés épars et bioturbation importante (pédotubules et chambres de diapause de vers de terre) (unité TN-4; fig. 9). La base de cette unité a livré une pointe paléolithique non patinée, séparée des précédentes par 10 à $15 \mathrm{~cm}$ de dépôt.

La partie supérieure de cette unité montre ensuite un passage assez progressif avec l'unité 13 , matérialisé par de très nombreuses traces de racines irrégulières à remplissage brun-sombre en provenance du sol 13 qui signent une deuxième phase de stabilisation du paysage (bioturbation intense par les racines et les terriers).

Ce sol 13 est représenté par un horizon $B_{\text {th }}$ typique des sols gris-forestiers de la France du Nord-Ouest (Antoine et al., 1994, 1999), et possède les caractéristiques typiques du sol SS-1 du complexe de sols de Saint- 
Sauflieu, dont le développement est attribué à l'interstade d'Odderade/Saint-Germain II (SI 5a) (Antoine et al., 1999). Dans la coupe du Chamesson, il se subdivise en trois horizons distincts sur le terrain, les unités 13 (fig. 36, $\mathrm{n}^{\circ \mathrm{s}} 1,2$ et fig. $38, \mathrm{n}^{\circ} 4-6$ ), 12 et 11 (fig. 8). L'analyse micromorphologique confirme cette observation et montre que l'horizon 12 (fig. $35, n^{\circ} 6$ ) correspond à la partie supérieure de 13 , toujours en place, mais fortement affectée par les processus de gel saisonnier profond (squelettanes abondants donnant une coloration plus claire sur le terrain), alors que l'horizon 11 (fig. $35, n^{\circ} 5$ ) représente un faciès déjà remobilisé par la gélifluxion du sol précédent. Néanmoins la succession des unités 13 à 11 ne traduit pas de changements majeurs de l'environnement et l'on peut considérer qu'elle s'inscrit dans la même oscillation climatique (variation de l'intensité relative des processus de gel-dégel, dans un contexte de fin d'interstade).

Les âges TL-IRSL obtenus dans cet horizon $(73,1$ $\pm 6,8$ ka BP pour l'unité 11 et $83,9 \pm 10$ ka BP au sommet de l'unité 13) confirment l'attribution de ce sol grisforestier complexe à l'interstade d'Odderade/SaintGermain II et sont en accord avec ceux obtenus dans le même niveau à Saint-Sauflieu (Engelmann, Frechen, 1998) et à Bettencourt-Saint-Ouen (Engelmann et al., 2000).

D'une manière générale, il faut néanmoins nuancer l'interprétation de la base du complexe de sols Début Glaciaire de Villiers-Adam (unités TN-6 à 11) en terme de succession de phases de stabilité et de pédogenèse séparées par des phases d'érosion et de colluvionnement. En effet, comme cela a déjà été observé dans d'autres complexes de sols comme à Bettencourt (Antoine, 2002), ces sols gris-forestiers du Début Glaciaire correspondent à des sols construits ou sols cumuliques (Almond, Tonkin, 1999), dont le développement pédologique est contemporain d'un apport sédimentaire (ruissellement diffus), progressivement incorporé au fond matriciel par la bioturbation et retouché par les processus d'illuviation argilo-humiques. La succession sol-colluvions visible sur le terrain traduit plus vraisemblablement une modification du bilan pédogenèse-dépôt colluvial qui évolue au cours du temps entre un maximum de colluvionnement lors des stades froids et un maximum de pédogenèse lors des interstades.

La limite supérieure de cette première partie du complexe de sols du Début Glaciaire (contact 11-10, Début Glaciaire A : Antoine et al., 1994) se marque dans tous les profils par un épisode majeur de gel saisonnier profond et par une phase d'érosion localement soulignée par un petit cailloutis. Cet épisode se traduit par la formation d'une forte structure polyédrique centimétrique à enduits silteux blancs (squeletanes), développée sur une épaisseur de 1 à $1,5 \mathrm{~m}$, qui recoupe tous les traits pédologiques antérieurs. Le drainage vertical de ces silts lavés, lors de phases de dégel et de fonte de la couverture nivale, provoque un important colmatage de la porosité et notamment des logettes de diapause (remplissage stratifié) (fig. $37, \mathrm{n}^{\circ}$ ) ). Cette phase de déstabilisation majeure avec accentuation des processus de gel-dégel est décrite dans la plupart des profils de l'Europe du Nord-Ouest (Haesaerts, 1985 ; Haesaerts et al., 1999; Antoine et al., 1998, 1999, 2001), où elle est responsable de la formation des "horizons blanchis " géliflués, comme ceux de Rocourt et de Rémicourt en Belgique (Haesaerts et al., 1999).

Elle est rapportée à la crise climatique de la fin du stade 5 a vers $75-73 \mathrm{ka} \mathrm{BP}$ (soit à la limite des stades isotopiques 5 et 4 ) et marque donc la limite avec la deuxième phase du Début Glaciaire, nettement plus aride (phase à sols steppiques).

La partie supérieure du complexe de sols du Début Glaciaire de Villiers-Adam Chauvry (unités 10 [fig. 8], TN-2 et TN-3 [fig. 9]) est relativement peu développée par comparaison avec les coupes de références du bassin de la Somme. Néanmoins elle montre aussi une nette évolution de la sédimentation vers un contexte où les apports éoliens locaux sont de plus en plus notables.

Le premier horizon rapportable à cette deuxième phase du Début Glaciaire est représenté par le niveau 10 visible dans tous les profils (fig. 33). Il se distingue nettement des sols précédents par l'absence totale de processus d'illuviation des argiles, par une texture nettement plus homogène "lœssique ", incorporant de fines papules des sols précédents (lames), par d'abondants micro-agrégats organiques intégrés à la masse (fig. 38, $n^{\circ} 3$ ), et par l'importance des carbonates secondaires: pseudomorphoses (cellules racinaires calcitées), et manchons racinaires de $\approx 1 \mathrm{~mm}$ de diamètre calcifiés (hypocoatings), qui traduisent une aridité relative du milieu (Beckzc-Deack el al., 1997). Ces caractéristiques traduisent des conditions climatiques plus froides et surtout plus arides, contemporaines de la fin du Début Glaciaire weichsélien (Début Glaciaire B : Antoine et al., 1994, 1999) ; une corrélation avec le sol SS-1 de Saint- 
Sauflieu est probable. Dans la coupe VA-99, cette partic du complexe de sols est un peu plus dilatée et incorpore un second horizon humifère qui présente sur le terrain et en lames minces le méme faciès de sol steppique (unité TN-2) (fig. 9). Cet horizon est, comme à SaintSauflieu (sol SS3b), séparé du précédent par un niveau peu épais de sédiment éolien à forte charge locale (unité TN-3) (fig. 9).

D’après la signature climatique de ces sols steppiques et leur position dans la séquence, il a été proposé (Antoine et al.. 1998, 1999) de les mettre en parallele avec les oscillations rapides situées vers 68 et $72 \mathrm{ka} \mathrm{BP}$ dans les séquences de glace GRIP et GISP II (interstades 19 et 20: Dansgaard ot al., 1993; Grootes ot al., 1993). Ces oscillations rapides (durèe $\leq 1000$ ans), également décrites dans les carottes marines de l'Atlantique Nord (Cortijo et al., 1995), traduisent une très grande instabilité climatique qui s'exprime clairement en domaine continental où la dynamique éolienne commence à se développer (Rousseau et al., 1998a, 1998b; Antoinc et al., 1999, 2001). Bien que la marge d'erreur qui affecte l'âge IRSL obtenu dans l'unité 10 (fig. 8 et 32), 72,2 $\pm 8,5$ ka BP (éch. 99-16), soit relativement importante, la valeur centrale est en accord avec ces interprétations, comme à Bettencourt Saint-Ouen (Engelmann ot al., 2000) et à Saint-Sauflieu (Engelmann, Frechen, 1998).

\section{Dépôts et sols du Pléniglaciaire weichsélien}

Ia première péjoration climatique majeure postéricure au Début (Blaciaire weichsélien se traduit par une érosion extrêmement marquée qui débouche localement sur la formation de vallons de 10 à $20 \mathrm{~m}$ de large pour 4 à $6 \mathrm{~m}$ de profondeur. Ces structures parallèes à la ligne de plus grande pente sont nettement visibles dans les coupes du Chamesson cntre les profils P3 et P4 (fig. 11) et dans la coupe VA-99 (fig. 12).

Cet épisode érosif qui représente la première signature du Plóniglaciaire inférieur est antérieur à la mise en place des limons sableux 9 datés de $50 \mathrm{ka}$ à 6) ka BP (fig. 32), dont l'épaisseur localement très importante est liée à une accumulation et à une préservation préférentielle dans les vallons qui fonctionnent alors comme des pièges sédimentaires.

Par ailleurs, de grandes structures dincision remplies par le sable limoncux 9 ont été obscrvées au Chamesson au sommet du complexe de sols humifère (fig. 14 et fig. 35, $\left.n^{\circ} 1\right)$. Leur géométrie, et leur morphologie qui découpe à "l'emporte pièce " le complexe de sol sousjacent, sont caractéristiques de chenaux de fonte liés à la dégradation d'un ancien réseau de coins de glace (thermokarst). Ces structures permettent donc de mettre clairement en évidence l'existence d'un permafrost au début du Pléniglaciaire inférieur.

Parallèlement, l'aridité du milieu est nettement soulignée par une variation brutale des valeurs de $\partial^{1: 3} \mathrm{C}$ à la limite 10/9 (valeurs beaucoup plus négatives en 9), des valeurs très basses de susceptibilité magnétique et la présence d'abondantes structures cellulaires végétales calcifiées (Beckze-Deack et al., 1997). Le contenu malacologique de cette unité, relativement pauvre, apparaît dominé par Pupilla muscorum, associée à quelques Trichia hispida et limaces. Il indique aussi un environnement de type steppe loessique. Par contre ce niveau est stérile en pollens probablement en raison de la granulométrie grossière du dépôt (oxýdation).

Dans ce contexte, la mise en place du dépôt sablolimoneux homogène 9 (sable fin > 50\%) (fig. 13) est alors essentiellement alimentée par le remaniement à courte distance (déflation) du substrat sableux local. Cependant la présence de carbonates primaires (contenant quelques tests de foraminifères) et de fractions limoneuses et argileuses non négligeables indique aussi des phénomènes de déflation affectant des sources plus lointaines (limons et sol plus anciens, affleurements calcaires).

\section{Pléniglaciaire moyen}

Dans les séquences de Villiers-Adam et de Chaury, le Pléniglaciaire moyen ou Interpléniglaciaire se caractérise par une certaine stabilité de l'environnement et le développement de pédogenèses peu éroluées de type sol brun boréal (Tedrow, 1977), ou Cambisol à Gelic Cambisol (FAO-UNESCO, 1997) et sols de prairie arctique (unités 8 à 3) (fig. 35, n 4). La première trace d'amélioration climatique attribuable au Pléniglaciaire moven est donc représentée par le développement d'un sol de type brun boréal, particulièrement bien développé dans la coupe du Chamesson et de VA-99 (unités 7 et 8) (fig. 35, n 4). Cet horizon se distingue par une coloration nettement plus brume bien visible sur le terrain, associée à une décarbonatation du dépôt précédent 9 et à une augmen- 
tation progressive de la teneur en argile qui passe de $11 \%$ à $24 \%$ au sommet de l'unité 7 (fig. 14). Cette évolution est accompagnée par une nette augmentation du pourcentage de carbone organique précédée par le retour vers des valeurs moins négatives des $\partial^{13} \mathrm{C}$ (fig. 14). Par ailleurs, les lames minces montrent un enrichissement du fond matriciel en argile et limon fins, ainsi qu'une augmentation du nombre des nodules ferromanganiques. Les processus pédologiques associés au développement de cet horizon sont essentiellement représentés par une décarbonatation suivie d'une "illuviation en masse " (illuviation mécanique liée aux processus de geldégel: Van Vliet-Lanoë, 1987) très différente des processus classiques d'illuviation dans les sols de type lessivés. On note également l'apparition de traces de bioturbation et des biosphéroïdes de calcite (nodules ovoïdes à structure fibroradiés de $\approx 500 \mu \mathrm{m}$ de diamètre) (fig. $38, \mathrm{n}^{\circ} 6$ ), attribuables à l'activité des vers de terre (Canti, 1998), et de nombreux manchons calcités en périphérie des biotubules (hypocoatings) (fig. $36, \mathrm{n}^{\circ} 5$ ).

L'ensemble de ces données traduit une augmentation progressive de la densité du couvert végétal, une stabilisation relative et une baisse de l'aridité du milieu permettant le développement de la faune du sol. Le contenu malacologique, bien que peu abondant (décarbonatation), est encore proche de celui de l'unité 9 et traduit en fait les conditions de dépôt du sédiment plus que celles du sol qui s'y est développé.

Les datations TL obtenues dans cette unité $(\approx 45-50$ ka BP) (fig. 32) confirment son attribution au Pléniglaciaire moyen et placent ce sol dans la partie inférieure du stade isotopique 3 ou au niveau des interstades 13-14 de GRIP que l on peut paralléliser avec le complexe de Moershoofd (Van der Hammen, 1995). Au plan régional, cet horizon peut être parallélisé avec le sol brun de Beauvais dans l'Oise (Locht et al., 1995b) et le sol de Saint-Acheul dans la Somme (Antoine, 1990).

La partie supérieure de ce sol se distingue ensuite par une augmentation de la densité des structures liées à l'activité biologique (biosphéroïdes, pédotubules, déjections : fig. $36, \mathrm{n}^{\circ} 6$ et fig. $38, \mathrm{n}^{\circ} 1$ ) et l'apparition d'une structure finement feuilletée de type gel-dégel de plus en plus nette. Parallèlement, les effectifs de mollusques sont plus importants et les assemblages se distinguent par l'apparition de Succinea oblonga indiquant une augmentation de l'humidité; le contenu pollinique, mieux conservé en raison d'une texture sédimentaire plus fine, permet de proposer une interprétation en terme de végétation (voir p. 31).

Ces données montrent l'image d'un milieu toujours assez ouvert (NAP : 60-70\%) de type toundra nordique, dominé par les taxons héliophiles et xérophiles où la végétation ligneuse semble essentiellement représentée par Juniperus (+ Pinus?).

Au sein du complexe pléniglaciaire de Villiers-Adam (unités 8 à 3) (fig. 8), l'unité 6 se distingue ensuite par un faciès de type sol de prairie arctique, plus humifère $(0,4 \%$ de COT), une augmentation de l'abondance des biosphédroïdes de calcite et une accentuation de la structure microfeuilletée (gel-dégel) (fig. $38, \mathrm{n}^{\circ} 2$ ). Le contenu malacologique est assez abondant et se distingue notamment par l'augmentation de Succinea oblonga, caractéristique des milieux humides. Dans l'unité 5 , on passe à un gley de toundra typique où l'humidité (stationnelle), liée à la couche active d'un permafrost, est très nette (abondance des succinées et présence de Ranunculaceae). La présence d'un permafrost développé à partir du sommet de la couche 5 est par ailleurs nettement attestée par la structure de gel-dégel typique qui affecte les unités 5 à 8 et par quelques structures de fusion avec érosion intense localisée (base de l'unité 4) (fig. 13).

L'ensemble de la séquence 8-5 correspond donc à une amélioration climatique relative suivie d'une nouvelle dégradation plus progressive qui aboutit au sommet de l'unité 5 à une érosion assez intense et à la mise en place de sables limoneux lités remaniant des éléments de sols antérieurs (unité 4).

La fin du complexe du Pléniglaciaire moyen se marque par la formation d'un deuxième horizon de type sol brun arctique qui a aussi été observé dans les profils de VA-99 et de Chauvry (unité 3). Cet horizon peu épais $\left(\approx 40-60 \mathrm{~cm}\right.$, horizon brun supérieur) (fig. $35, \mathrm{n}^{\circ} 4$ ) est cependant beaucoup moins développé que celui de l'unité 8 et en partie érodé lors de la crise de la base du Pléniglaciaire supérieur. Cette unité est caractérisée par une activité biologique assez marquée (déjections, biosphéroïdes de vers) et un contenu malacologique beaucoup plus riche que celui des lœss sus-jacents (voir p. 37).

La succession pédostratigraphique du complexe interpléniglaciaire de Villiers-Adam/Chauvry présente par ailleurs une certaine analogie avec celle décrite dans 
la vallée du Rhin à Nussloch, qui se caractérise aussi par la présente d'un complexe pédologique montrant un minimum de deux sol bruns (Cambisols) entre $\approx 45 \mathrm{ka}$ et 30 ka BP (Antoine et al., 2001; Schirmer, 2000). Par contre, dans la plupart des autres profils de la France septentrionale, le bilan pédosédimentaire correspondant à cette période est beaucoup plus réduit et uniquement représenté par un horizon de sol brun polyphasé à structure lamellaire fine et abondantes traces de carbonate secondaire (sol de Saint-Acheul: Antoine, 1990). Compte tenu de ces données et des corrélations que l'on peut faire avec des séquences datées dans les régions limitrophes, les âges TL-IRSL obtenus dans la partie supérieure du complexe de Villiers-Adam (unités 3 à 5) semblent nettement sous-estimés.

\section{Pléniglaciaire supérieur}

La limite avec le Pléniglaciaire supérieur est définie par la disparition définitive des sols bruns précédemment décrits, par une crise érosive majeure et surtout par la mise en place d'une couverture généralisée de loess carbonatés allochtones (Antoine et al., 2001).

Dans les séquences de Villiers-Adam, la crise érosive du début du Pléniglaciaire supérieur, que l'on peut dater d'environ $30 \mathrm{ka}$ BP par comparaison avec les données des régions avoisinantes (Antoine et al., 1999, 2001), se manifeste d'une manière très intense, avec la formation d'une dernière génération de vallons parallèles à la pente du versant (fig. 11, base P5-P6 et fig. 12, base de 2d). La formation de ces structures, plus localisées et un peu moins profondes (2-3 $\mathrm{m}$ ) que celles du Pléniglaciaire inférieur, est liée à la dégradation d'un réseau de grands coins de glace (processus thermokarstiques) qui, bien que dégradés, apparaissent encore nettement à la base du colmatage lossique dans l'axe des vallons (fig. 12). La présence d'un niveau d'icewedges s'ouvrant au sommet des sols du Pléniglaciaire moyen est d'ailleurs une caractéristique des lœess du Nord-Ouest européen (Haesaerts et al., 1999 ; Antoine et al., 1998, 1999).

Le secteur de Villiers-Adam se situe à la limite méridionale d'extension de la zone lossique (fig. 2), dans une zone où les lœess du Pléniglaciaire supérieur sont en moyenne très peu épais, sauf ponctuellement en position de couverture de terrasses sur la rive droite de l'Oise (Lebret, Halbout, 1991). Cependant, l'analyse des séquences de Villiers-Adam/Chauvry, surtout dans le profil VA-99 (fig. 12), permet de reconnaître sans difficulté les traits principaux du bilan du Pléniglaciaire supérieur de la France septentrionale (Antoine et al., 1999) :

- lœss calcaire typique homogène (2d) ;

- loess calcaires lités à microfentes de dessiccation (2c) ;

- horizon à langues de gélifluxion grisâtres (cf. horizon de Nagelbeek) (2b) ;

- loess calcaire homogène (loess de couverture) (2a).

D'une manière générale, ces faciès lœssiques du Pléniglaciaire supérieur se distinguent nettement des dépôts antérieurs par leur faciès nettement carbonaté $\left(\mathrm{CaCO}_{3} \approx 15 \%\right)$, l'importance de la fraction $20-50 \mu \mathrm{m}$ typique des loess (50-65\%), des valeurs de $\partial^{13} \mathrm{C}$ moins négatives (aridité) (Hatté et al., 1998) et des valeurs de susceptibilité très basses typiques de ces dépôts (Antoine et al., 1999 ; Rousseau et al., 1998a, 1998b). On observe aussi, dès la base de ces losss, un pourcentage très faible de carbone organique et une très forte réduction des effectifs malacologiques que l'on peut interpréter comme la trace d'un paysage très peu végétalisé de type steppe froide lœssique, peu propice au développement des malacofaunes.

Néanmoins, au sein de ces lœss, le niveau de gley géliflué $(2 b)$, qui sépare les deux dernières unités, se distingue par des valeurs de carbone organique plus élevées et un $\partial^{13} \mathrm{C}$ plus négatif, indiquant un contexte plus humide. Cette humidité relative, liée à une imperméabilisation superficielle par le permafrost (stationnelle), est aussi plus favorable au développement de la végétation et surtout des mollusques qui sont nettement plus nombreux dans cet horizon. Cependant aucune amélioration climatique n'est détectée à ce niveau. En raison de sa position dans la stratigraphie, à l'interface entre les lœess calcaires lités 2c et les loess homogènes $2 a$, cet horizon pourrait correspondre à l'horizon à langues de Nagelbeek (Kesselt) qui jalonne cette limite dans la majeure partie des profils de la Belgique à la Normandie et qui est daté d'environ $22 \mathrm{ka} \mathrm{BP}{ }^{14} \mathrm{C}$ (Sommé et al., 1980 ; Lautridou, 1985).

Enfin il faut souligner que les caractéristiques sédimentologiques et bioclimatiques de ces loess calcaires du Pléniglaciaire supérieur weichsélien, déposés entre $30 \mathrm{ka}$ et $15 \mathrm{ka} \mathrm{BP}$ environ, sont très proches de celles des loess calcaires de la fin du Saalien.

Cette similitude, observée d'une manière plus générale dans toute la France septentrionale (Antoine, 
1990) et dans la vallée du Rhin (observation personnelle), permet de suggérer des conditions climatiques et des zones sources identiques pour ces deux périodes froides majeures de la fin du Pléistocène.

La fin de la séquence 3 est caractérisée par le retour de conditions tempérées interglaciaires attestées par l'horizon $\mathrm{B}_{t}$ de sol brun lessivé de l'unité 1 , dont le développement est attribuable au bilan du Tardiglaciaire weichsélien et de l'Holocène (Van Vliet-Lanoë, 1987).

\section{CHRONOSTRATIGRAPHIE ET ENVIRONNEMENT DU SITE DE VILLIERS-ADAM, LE PETIT SAULE}

\section{INSERTION DANS LA SÉQUENCE PÉDOSTRATIGRAPHIQUE ET PALÉOCLIMATIQUE}

L'étude stratigraphique et paléopédologique des coupes de Villiers-Adam permet donc de mettre en évidence une succession de séquences pédosédimentaires de type Glaciaire-Interglaciaire, dont la composition et la logique sont tout à fait comparables à celles décrites dans les régions avoisinantes (Antoine et al., 1998, 1999).

Sur ces bases, et compte tenu des données géochronologiques obtenues sur des séquences similaires dans les autres régions (Antoine et al., 1998 ; Engelmann et al., 2000), il a donc été possible, dans un premier temps, de positionner sans grande difficulté les occupations humaines de Villiers-Adam au sein des phases initiales du Début Glaciaire weichsélien, et de leur attribuer un d'âge compris entre 112 ka et 75 ka BP (Antoine, in Locht et al., 1997). Les résultats de la campagne de datation TL-IRSL menée sur le site par M. Frechen et les dates TL sur silex obtenues par $\mathrm{N}$. Debenham sur le niveau archéologique confirment par ailleurs ces premières interprétations chronostratigraphiques. D'une manière plus détaillée, les observations de terrain montrent que le niveau paléolithique principal du Petit Saule se localise au sein de dépôts à dominante colluviale qui correspondent à un bilan pédosédimentaire complexe et peu épais (fig. 33 : unité PSF, niveau archéologique), par comparaison aux enregistrements plus complets de VA-99 et du Chamesson (fig. 33).

Les observations de terrain montrent que cette unité PSF, localement surmontée par une unité (sol gris- forestier fortement dégradé, géliflué) comparable au complexe 12-11 (fig. 33), est en revanche systématiquement antérieure à un sol isohumique steppique (unité 10).

Les données micromorphologiques confirment ces observations et montrent clairement au sein de l'unité PSF la présence d'une phase d'illuviation silto-humique stratifiée semblable à celle rencontrée dans les lames des unités 13-12 (sol gris-forestier) (fig. 37, $\mathrm{n}^{\text {os }} 4$ à 6).

Cette phase est cependant très fortement fracturée par un épisode majeur de gel saisonnier profond, responsable de la structure fortement polyédrique de l'unité PSF, de sa déstructuration localement assez poussée (début de cryoreptation, intense fracturation des revêtements argileux) (fig. 37, $\mathrm{n}^{\circ} 6$ ) et de l'importante percolation de silts blancs lavés (siltanes) que l'on y observe (fig. $37, n^{\circ} 5$ ).

Compte tenu des données précédemment exposées (voir p. 56), cette péjoration climatique importante, antérieure à la phase à sols steppiques, est aisément parallélisable avec la phase majeure de gel saisonnier qui termine systématiquement la phase à sols grisforestiers du Début Glaciaire A vers $73 \mathrm{ka}-75 \mathrm{ka} \mathrm{BP}$ (fin du stade $5 \mathrm{a}$ ). Ces observations permettent de resserrer la fourchette chronologique vers le bas, en montrant que la mise en place des colluvions limoneuses de l'unité PSF est antérieure à l'interstade d'Odderade/ Saint-Germain II ( $\approx 75 \mathrm{ka}-85 \mathrm{ka} \mathrm{BP})$.

Par ailleurs, les données micromorphologiques montrent que cette unité PSF contient de très nombreuses concrétions (nodules) ferromanganiques et des fragments de charbon de bois remaniés (arrondis) provenant du remaniement des sols antérieurs. Sur le terrain, le caractère colluvial de ce niveau est aussi marqué par la présence de gros nodules remaniés épars plus sombres (diamètre $\approx 1 \mathrm{~cm}$ ) qui attestent du remaniement d'un sol plus ancien. Par ailleurs, cette unité se distingue aussi par la présence de nombreuses traces de racines (bioturbations) de grande taille (centimétriques) à remplissage aussi plus sombre et plus humifère, qui témoigne du développement d'un second sol grisforestier ultérieurement érodé (voir lames minces).

Dans une phase initiale de l'étude du site, ces dernières observations avaient débouché sur une première tentative de corrélation avec la coupe de référence du Chamesson dans laquelle l'unité PSF représentait un équivalent de l'unité 12 . Cette première inter- 
prétation situait alors l'industrie entre les interstades de Brørup/Saint-Germain I et d'Odderade/SaintGermain II, vers 90 ka-75 ka BP (Antoine, in Bahain et al., 1996).

Les datations TL sur silex ainsi que la découverte des nouveaux profils de VA-99 et de la séquence très complète de Bettencourt-Saint-Ouen ont permis d'affiner cette interprétation. En effet, dans le profil VA-99 comme à Bettencourt (Somme), il a été possible de mettre en évidence les phases initiales du Début Glaciaire (Herning/Melisey I-Brørup/Saint-Germain I), antérieures au sol gris-forestier typique SS-1 qui constitue généralement le terme le plus ancien du bilan (Antoine et al., 1999).

Ces observations permettent de mieux situer la formation du niveau PSF. En effet, si dans la coupe du Chamesson il n'existe pas de faciès équivalent à l'unité PSF, le profil VA-99 montre en revanche, sous le sol gris-forestier 13, une unité originale dont les caractéristiques macro- et microscopiques sont très proches de celle de l'unité PSF.

Cette unité (TN-4) (fig. 9 et 33) présente en effet un faciès de colluvions limono-sableuses à nodules de sol remaniés, microcharbons et débris humifiés, intégrant une phase d'illuviation argilo-humique fortement fracturée et une phase finale de gel saisonnier profond avec production massive de silts lavés, dont les caractéristiques sont très proches de celles observées dans PSF. On remarque par ailleurs que quelques artefacts non patinés, qui pourraient se raccorder à ceux du Petit Saule, ont été récoltés à la base de l'unité TN-4 lors de l'étude du profil Pl de VA-99.

Compte tenu de l'interprétation chronostratigraphique proposée pour cette partie de la séquence à Villiers-Adam (voir p. 17) et à Bettencourt (Antoine, in Locht dir., 2002), ces nouvelles observations nous permettent de situer l'occupation paléolithique à la fin de l'interstade de Brørup/Saint-Germain I, soit vers $100 \mathrm{ka}$ BP (fig. 34), en accord relatif (compte tenu des marges d'erreur) avec les âges TL obtenus pour le niveau paléolithique de Villiers-Adam (moyenne: $105 \mathrm{ka}$ \pm 12 ka BP) ${ }^{3}$ (fig. 32).

3. Trois silex chauffés provenant du secteur 1 ont été datés par thermoluminescence (TI.) par Nick Debenham. Les pièces datées ont ceté choisies dans un groupe de dix-huit silex dont les émissions TI. ont cté examinées, ce qui a permis d'observer que la plupart d'entre cux n'araient pas été chauffés à des températures suffisamment élevées
Les âges TL sur sédiment, obtenus sur l'unité PSF au niveau de la fouille, apparaissent en revanche asse\% fortement sous-estimés (moyenne autour de 70,4 ka BP). Néanmoins il faut souligner que le niveau PSF a subi un très fort impact du gel-dégel aux environs de $70 \mathrm{ka} \mathrm{BP}$, responsable d'une intense accumulation silteuse. Ces silts fins, en partie issus de particules lavées en surface par la fonte de la couverture nivale et qui ont percolé dans l'ensemble de l'unité PSF, sont peut-être à l'origine de ce rajeunissement, comme cela a été signalé à BettencourtSaint-Ouen (Antoine, 2002).

\section{ENVIRONNEMENT, CONSERVATION DU NIVEAU ARCHÉOLOGIQUE (TAPHONOMIE)}

En l'absence de données palynologiques directement associées (voir p. 20), l'interprétation du contexte environnemental contemporain des occupations paléolithiques du Petit Saule est particulièrement délicate et peut uniquement se fonder sur les données paléopédologiques et l'étude des lames minces. Néanmoins la corrélation avec les autres complexes de sols Début Glaciaire ayant fait l'objet de résultats palynologiques comme ceux de Saint-Sauflieu et de Bettencourt et le croisement avec les données paléopédologiques permettent de proposer pour Villiers-Adam une interprétation cohérente.

Les artefacts en silex qui constituent le niveau archéologique sont essentiellement conservés à la base de l'unité dénommée PSF pour les plus grosses pièces et les amas de débitage, alors que les autres pièces apparaissent dispersées dans la masse de cette unité sur environ 0,2 m d'épaisseur (fig. 39).

Ces observations, associées à l'absence d'esquilles, indiquent une certaine perturbation de la nappe d'artefacts originels. Compte tenu de la pente de la surface topographique où se sont installés les Paléolithiques (de $5 \%$ à $8 \%$ dans le secteur 1 et de $15 \%$ à $20 \%$ dans les deux autres secteurs), l'absence quasi totale d'esquilles, même dans les amas de débitage, montre que le niveau

pour autoriser une détermination d'âge. I es trois silex choisis, indexés 994, 5433 et 406 ont été respectivement datés de $132 \pm 19 \mathrm{ka} ; 107 \pm 13 \mathrm{ka}$ et $103 \pm 12 \mathrm{ka}$ étant dommé les incertitudes de mesure des âges TI. et si l'on estime que ces pièces ont toutes été chauffées au mêne moment. la meilleure estimation d’àge pour cet événement est de 110 \pm 11 ka BP ou de $105 \pm 12$ ka BP si l'on excepte le resultat obtenu sur le silex 994 . 


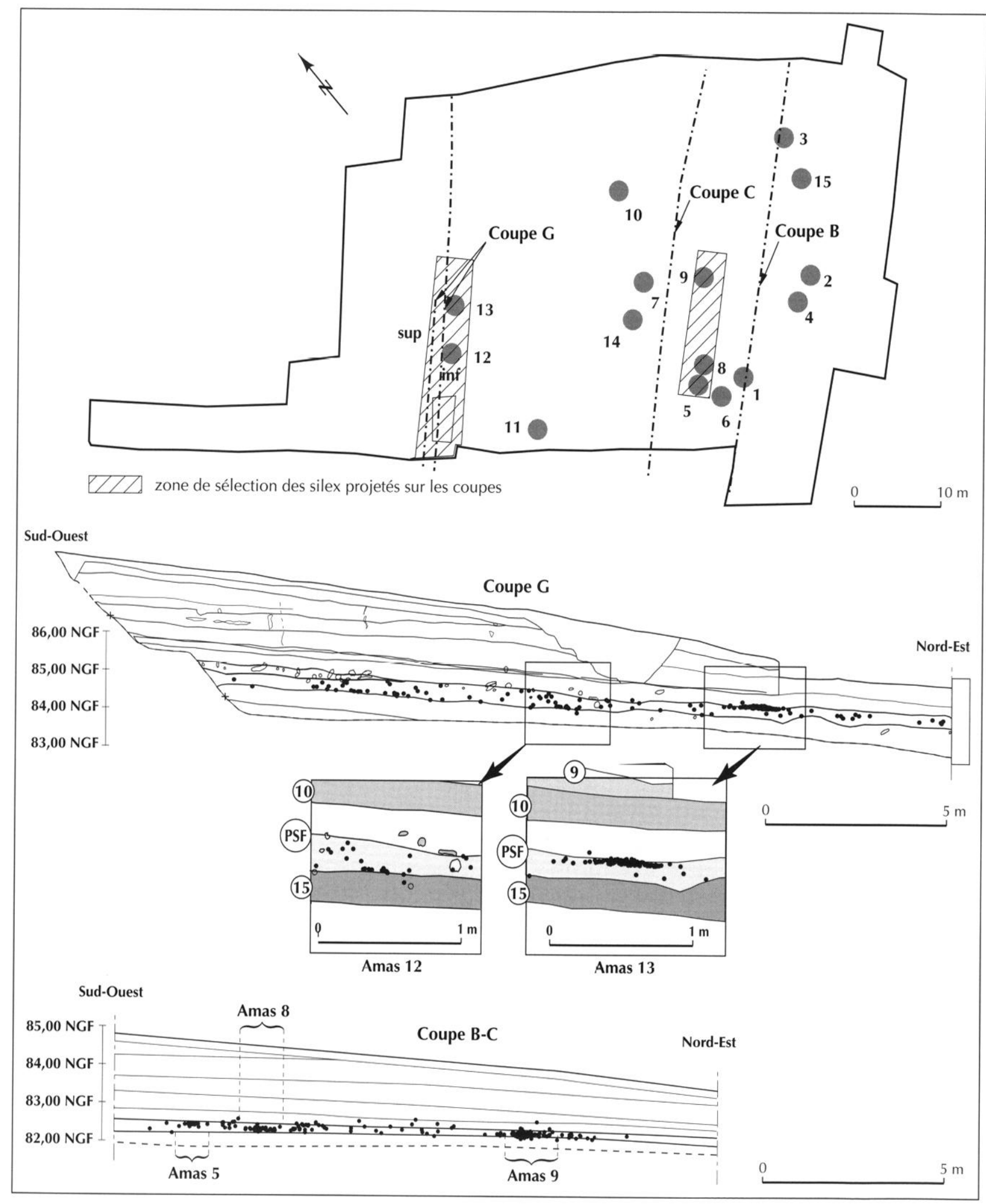

Fig. 39 - Projection verticale des artefacts du niveau 2 (dessin P. Raymond). 
archéologique a été affecté par des processus de ruissellement relativement importants. Néanmoins les artefacts de plus grandes dimensions semblent ne pas avoir été significativement affectés par ce phénomène. Certains gros éclats ont été gélifractés sur place et les fragments sont restés côte à côte (fig. 40). Par ailleurs, d'après les informations issues des remontages, les amas de débitage semblent également en position quasi primaire.

Compte tenu de ces observations et des données précédemment exposées sur l'interprétation des phénomènes pédosédimentaires en termes paléoclimatiques, il est possible de replacer les occupations paléolithiques du Petit Saule dans l'évolution suivante (fig. 34).

1. Stade de Herning/Melisey 1 (5d): au cours des phases initiales du Début Glaciaire, troncature du $B_{1}$ éémien avec production d'une première génération de colluvions à charbons de bois épars piégées en contrebas du site.

2. Interstade de Brørup/Saint-Germain I (5c) : stabilisation relative du paysage dans une ambiance forestière de type forêt boréale (Betula, Pinus dominants) avec toujours quelques thermophiles, absence de dépôt au niveau du site, surimposition d'une nouvelle pédogenèse dans le $B_{t} 15 a$, occupation du site par les Paléolithiques. Les conditions climatiques sont alors caractérisées par une nette continentalité : températures estivales légèrement inférieures à l'actuel (moins $2^{\circ}$ à $3{ }^{\circ} \mathrm{C}$ : Lowe, Walker, 1997), saison hivernale plus longue et températures minimales nettement plus basses favorisant les processus de gel en profondeur (>-1 m) et le développement de la couverture nivale.

Compte tenu des dates TL obtenues sur silex chauffé, l'occupation de Villiers-Adam (Petit Saule) se placerait au niveau de l'optimum climatique, qui apparaît dans la courbe isotopique du $\partial^{18} \mathrm{O}$ de GRIP vers $100 \mathrm{ka}-107 \mathrm{ka}$ BP environ (fig. 34) ou dans le début de la phase de dégradation climatique progressive qui suit. La position stratigraphique des quelques artefacts découverts dans la séquence de VA-99 au sommet du sol de l'unité TN-5 et à la base de l'unité colluviale TN-4 (fig. 9) est en accord avec cette interprétation.

3. Stade de Rederstall/Melisey II ( $5 b$ ) : cette nouvelle dégradation climatique provoque une ouverture très nette du paysage ; l'érosion des sols reprend et entraîne la mise en place d'une nouvelle génération de colluvions (unité TN-4). Au cours de cette phase où les processus de

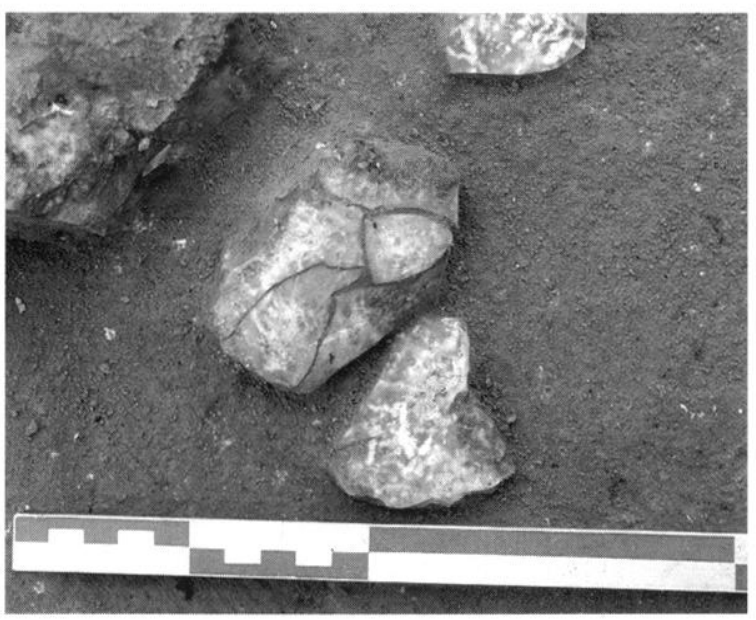

Fig. 40 - Villiers-Adam, Le Petit Saule : éclat gélifracté sur place (photos P. Raymond).

ruissellement redeviennent très actifs, le secteur occupé préalablement par les Paléolithiques, situé sur une pente assez marquée, est soumis à une érosion intense qui entraine le déplacement des esquilles et des petites pièces. Parallèlement, une partie des artefacts non recouverts par des sédiments acquiert une patine assez. marquée et subit l'action des périodes de gel particulièrement intense (artefacts gelifractés). L'accumulation d'une couche de colluvions limono-argileuses issues du remaniement des sols précédents débouche sur la formation de l'unité PSF contenant de nombreuses pièces paléolithiques.

D'après la courbe du $\partial^{18} \mathrm{O}$ de GRIP, cette période de dégradation climatique progressive aurait duré un peu plus de 10000 ans, avec un paroxysme vers $90 \mathrm{ka} \mathrm{BP}$ (fig. 34).

4. Interstade d'Odderade/Saint-Germain II (5a) : l'amélioration climatique contemporaine de cet interstade correspond à un retour de conditions proches de celles qui existaient lors de l'interstade de Brørup/SaintGermain I, au cours d'une période qui aurait duré 7000 à 8000 ans (fig. 34). Du point de vue pédologique, cette phase se distingue nettement par la formation du sol gris-forestier typique (unité 13), développé sur colluvions dans les secteurs de bas de versant. Au niveau du site, on observe seulement le développement d'une phase de pédogenèse qui affecte l'unité PSF et le sommet du $B_{1} 15$.

5. Phase froide finale du stade 5a : au cours de cette dégradation climatique majeure, le secteur du site paléolithique est à nouveau soumis à une érosion généralisée 
qui décape entièrement le sol gris-forestier ainsi que la partie supérieure de PSF. Parallèlement, les processus de gel-dégel particulièrement intenses entraînent une nouvelle phase de déstabilisation de l'unité PSF contenant les artefacts (gel-dégel, début de cryoreptation), ainsi qu'une très intense fragmentation des traits pédologiques hérités des phases antérieures.

Cette interprétation chronostratigraphique proposée pour les occupations humaines de Villiers-Adam repose sur l'ensemble des données actuellement disponibles pour le site de Villiers-Adam et la comparaison avec celles du bassin de la Somme et du nord de la France.

Néanmoins, pour l'un d'entre nous (P. Haesaerts), une autre hypothèse de corrélation avec les enregistrements globaux est possible par comparaison avec les séquences de Belgique et de Rhénanie. Dans ce schéma, l'ensemble de la séquence pédosédimentaire TN-6 à 13 est corrélable avec la succession des sous-stades isotopiques $5 \mathrm{~d}$ à $5 \mathrm{c}$ (interstades 22 à 24 de GRIP) (fig. 34) ; le sol steppique de l'unité 10 correspondant alors au deuxième interstade majeur (Odderade/SaintGermain II GRIP-21). Cependant, compte tenu des marges d'erreur qui caractérisent les données géochronologiques, cette deuxième hypothèse n'a pas d'impact majeur sur la position chronostratigraphique des niveaux paléolithiques dont l'âge est toujours d'environ $100 \mathrm{ka} \mathrm{BP}$.

Enfin, d'une manière générale, les hypothèses quant à la répartition spatiale des vestiges et à leurs éventuels déplacements dus à l'homme doivent donc tenir compte de l'enchaînement des phases pédosédimentaires et climatiques résumées précédemment. Par ailleurs, en raison des ravinements d'âges divers qui affectent le niveau $\mathrm{N} 2$ et bien que les artefacts se trouvent en position stratigraphique similaire, toute corrélation directe pour les niveaux du Début Glaciaire weichsélien entre les différents secteurs de fouille est délicate. La stricte contemporanéité des séries récoltées dans chacun de ces trois secteurs n'est donc pas établie avec certitude et les trois ensembles lithiques ont donc été étudiés individuellement, tant sur le plan technotypologique que sur celui de l'analyse spatiale.

Fig. 41 - Villiers-Adam, Le Petit Saule : localisation des différents secteurs de la fouille et du matériel du niveau 2 (dessin P. Raymond).

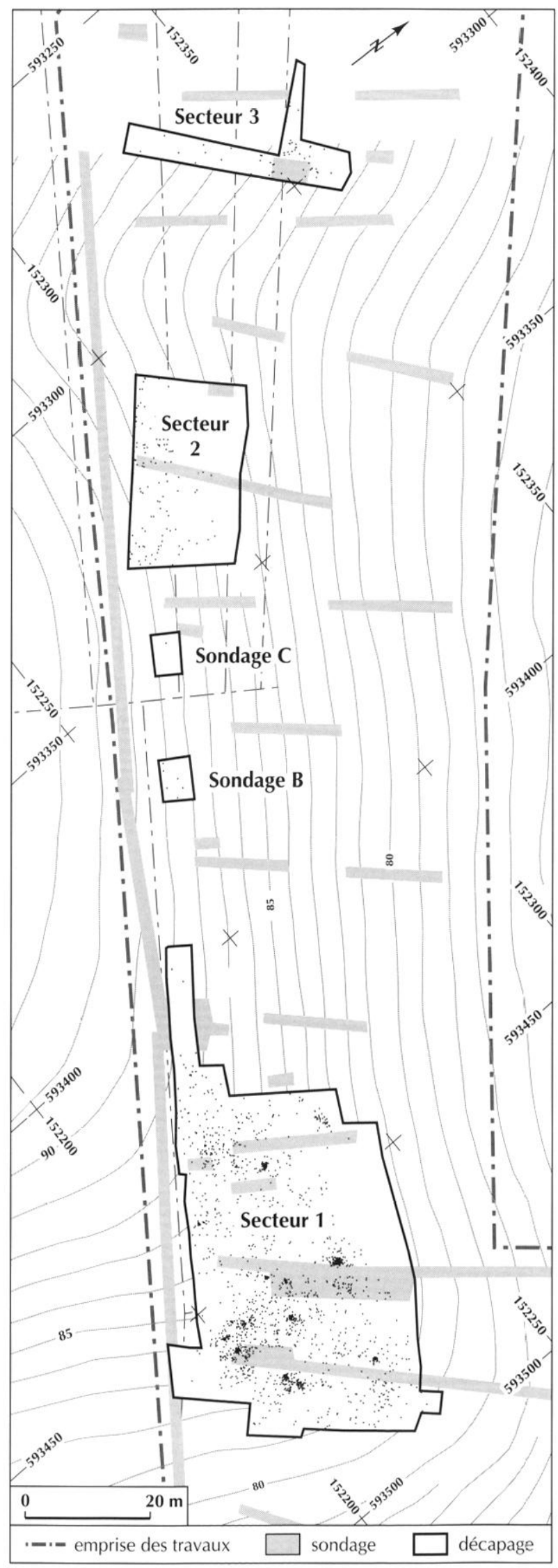




\section{ÉTUDES ARCHÉOLOGIQUES}

\section{MÉTHODES D'INTERVENTION ET ÉTENDUE DU GISEMENT}

La phase d'évaluation avait permis d'estimer la superficie du gisement concernée par l'emprise autoroutière à $4300 \mathrm{~m}^{2}$ et de mettre en évidence deux secteurs séparés par une zone démantelée par ravinement (Locht et al., 1997). Un troisième secteur, situé à l'ouest des deux premiers, a été repéré durant la fouille (fig. 41).

Dans les trois secteurs, les artefacts se trouvent en position stratigraphique similaire, sans qu'il soit possible d'établir de liaison stratigraphique entre ces trois secteurs. Les ensembles lithiques ont été traités de façon séparée, puisque la contemporanéité de ces trois séries ne peut être établie avec certitude par les études stratigraphiques et micromorphologiques.

En raison de la faible densité du matériel lithique, une intervention à la pelle mécanique, déjà utilisée dans des contextes similaires (Deloze et al., 1994), a été préconisée sur le niveau le plus riche, les deux autres ensembles ne comprenant que quelques artefacts. La totalité de la surface explorée de la sorte s'élève à $3866 \mathrm{~m}^{2}$, divisés en trois secteurs de dimensions et de densités différentes (tabl. X), auxquels il faut ajouter les sondages $B$ et $\mathrm{C}$, respectivement de $49 \mathrm{~m}^{2}$ et $42 \mathrm{~m}^{2}$, où le niveau archéologique est démantelé.

Les zones les plus denses, tels les amas de débitage, ont été fouillées de façon classique. Au total, $107 \mathrm{~m}^{2}$

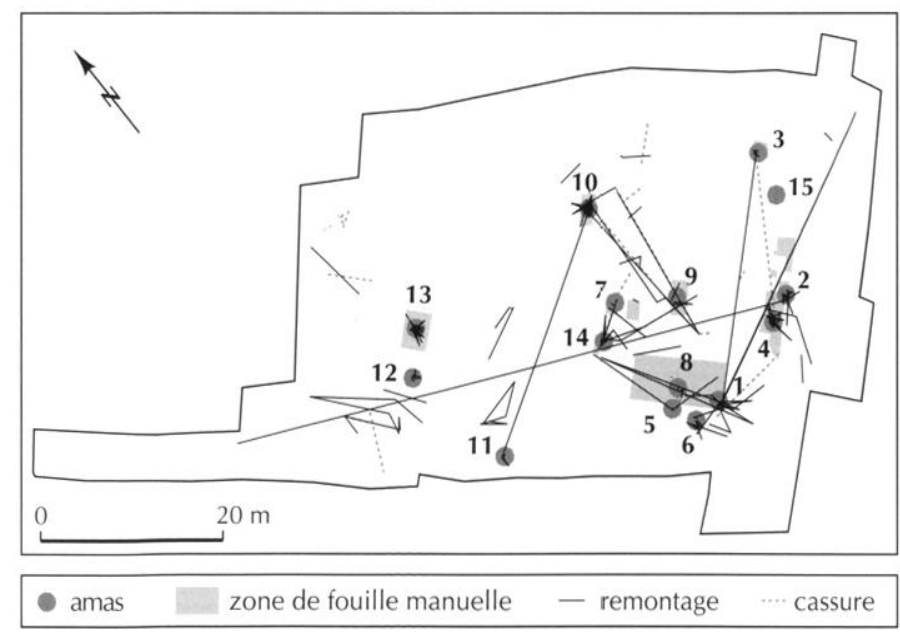

Fig. 42 - Villiers-Adam, Le Petit Saule: localisation des amas de débitage et des remontages (dessin P. Raymond).

\begin{tabular}{|c|c|}
\hline Zone fouillée & Superficie \\
\hline Secteur 1 & $2813,24 \mathrm{~m}^{2}$ \\
\hline Secteur 2 & $750,78 \mathrm{~m}^{2}$ \\
\hline Secteur 3 & $302,67 \mathrm{~m}^{2}$ \\
\hline Total & $\mathbf{3 8 6 6 , 6 9} \mathbf{~ m}^{\mathbf{2}}$ \\
\hline
\end{tabular}

Tabl. $\mathbf{X}$ - Superficie des differrents secteurs fouillés au Petit Saule à Villiers-Adam.

localisés dans le secteur 1 ont été fouillés à la main, soit $3,66 \%$ de la superficie fouillée (fig. 42). Les amas fouillés manuellement ont été relevés au $1 / 10$ de façon classique (fig. 43).

Chaque pièce lithique découverte a été aussitôt positionnée précisément en trois dimensions au moyen d'un théodolite électronique et replacée en coordonnées Lambert. L'ensemble des informations a été transféré quotidiennement sur un Système d'information géographique (SIG). Ces informations s'intègrent sur une base contenant un extrait des parcelles et emprises parcellaires de la liaison Cergy-Roissy, les courbes de niveau, etc. L'impression rapide de plans à échelle variable a fourni la possibilité de visualiser, au jour le jour, l'évolution du décapage. La densité des zones explorées a permis d'orienter au quotidien la stratégie de fouille vers les endroits les plus denses. La fouille des secteurs 2 et 3, assez pauvres en matériel, a ainsi été délaissée assez rapidement au profit du secteur 1 , qui constitue la zone la plus riche en artefacts et en concentrations significatives (amas) (fig. 44).

Dans le secteur l, le niveau archéologique a été démantelé par érosion au nord-est et au sud-est. En

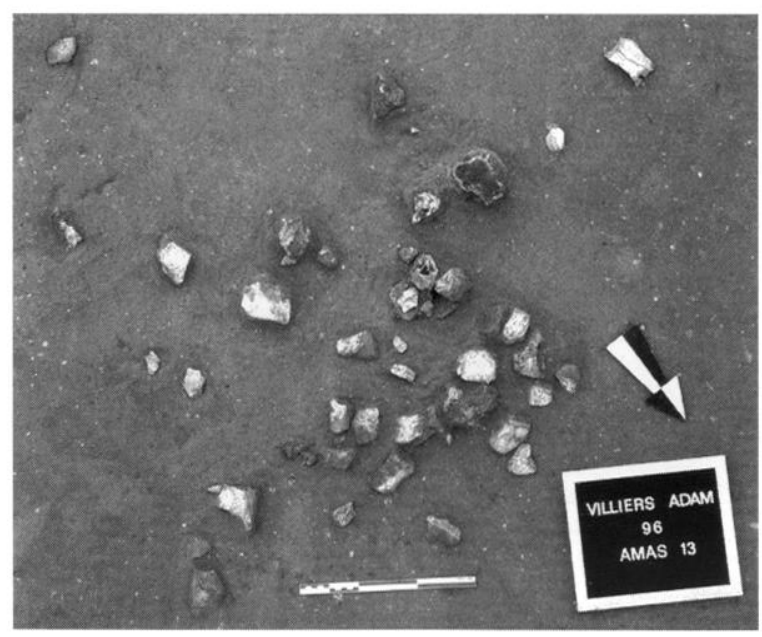

Fig. 43 - Villiers-Adam, Le Petit Saule : amas 13 en rours de fouille (photos P. Raymond). 
revanche, il se poursuit au sud, sous le talus de la future voie rapide. De petits vallons fossiles d'érosion entaillent de plus ce secteur, mais n'affectent le niveau archéologique que de façon superficielle. Soixante-quinze

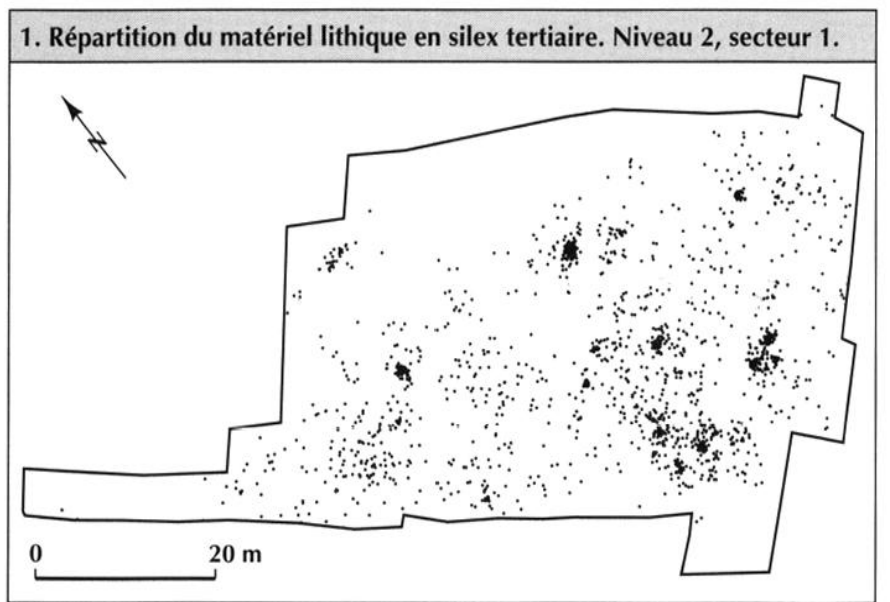

2. Répartition du matériel lithique en silex secondaire. Niveau 2 , secteur 1.

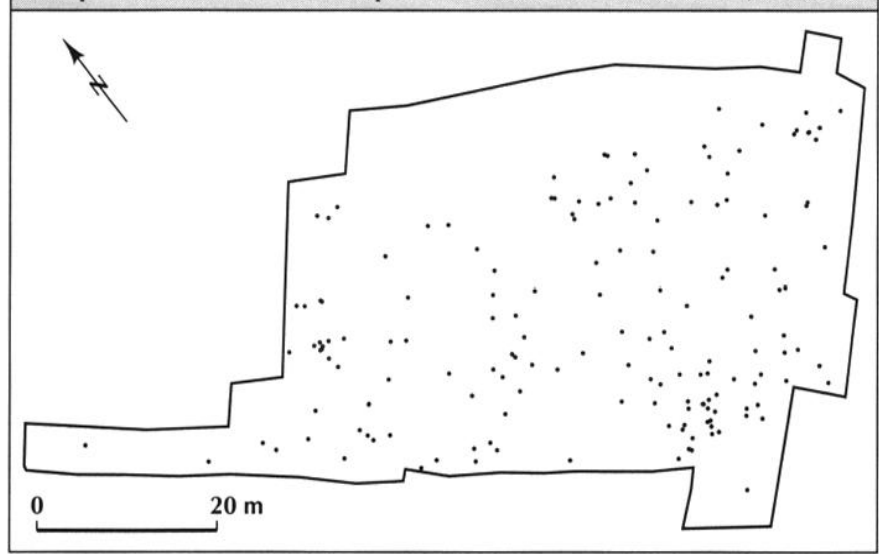

3. Répartition du matériel lithique en grès. Niveau 2, secteur 1.

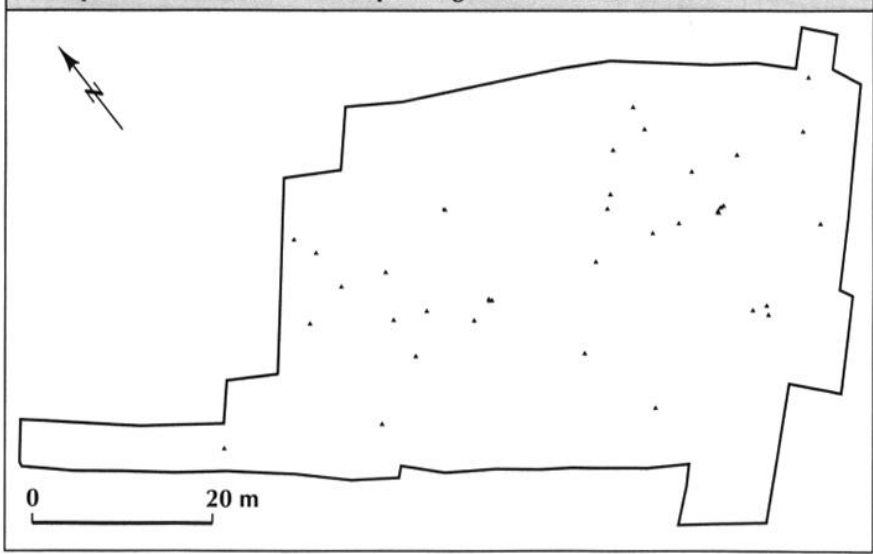

Fig. 44 - Villiers-Adam, Le Petit Saule : localisation du matériel archéologique par type de matériau. 1, silex tertiaire ; 2 , silex secondaire; 3 , grès. artefacts (nucléus, lames, pointes et éclats Levallois, etc.) ont été récoltés dans les sables jaunes qui colmatent ces ravinements. Ces silex proviennent du niveau d'occupation du Weichsélien ancien, mais sont en position remaniée. Seul un décompte est présenté à titre indicatif.

Les secteurs 2 et 3 ont été conservés de façon identique au secteur 1, mais la densité du matériel

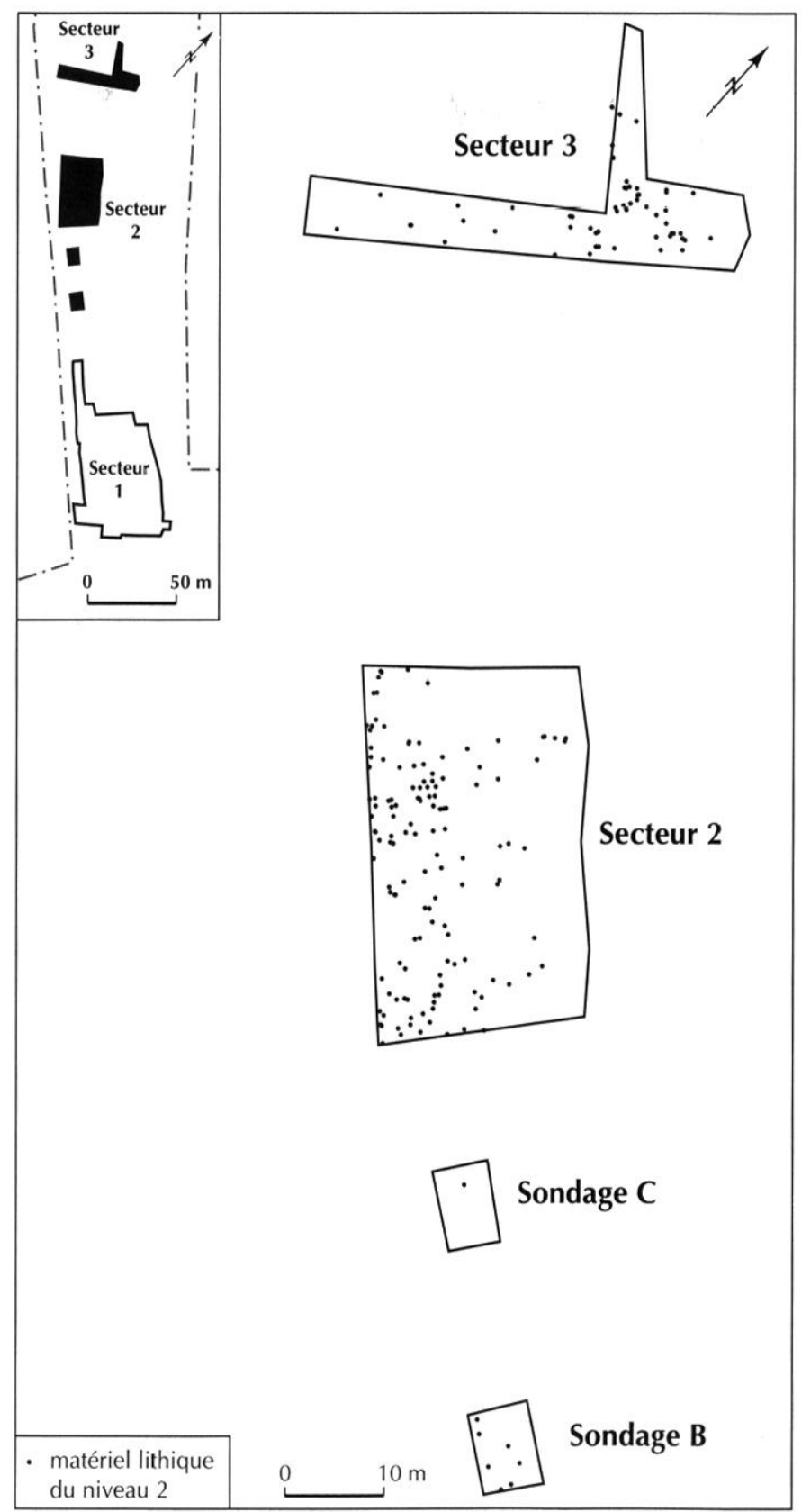

Fig. 45 - Villiers-Adam, Le Petit Saule: localisation du matériel archéologique dans les secteurs 2 et 3. 
lithique est très faible (fig. 45). Ces deux occupations sont érodées au nord-est, mais, tout comme dans le secteur 1, le niveau archéologique semble se poursuivre en direction du sud-ouest, sous le talus de la future voie rapide.

\section{LA MATIÈRE PREMIÈRE}

Trois types de matériau ont été principalement utilisés par les tailleurs paléolithiques de VilliersAdam (fig. 46) : le silex tertiaire du calcaire de SaintOuen (Marinésien, Éocène supérieur) ; le silex secondaire de la craie de Beauvais (Campanien, Crétacé supérieur) et le grès de Beauchamp (Auversien, Éocène supérieur).

\section{PÉTROGRAPHIE DES MATIÈreS PREMIÈRES}

Les différents types de matières premières présentent divers aspects macroscopiques en fonction de leur origine et de leur patine. Chaque faciès observé est décrit matière par matière.

Le silex tertiaire présente deux faciès principaux. Le premier correspond à un silex blond ou brun de qualité moyenne, présentant parfois des reflets rougeâtres ou violacés, translucide, compact, à grains fins et à cortex assez épais pouvant atteindre plusieurs centimètres (fig. $46, n^{\circ} 3$ ). Les blocs sont soit d'assez grande taille, de forme oblongue et présentant parfois des zonations plus ou moins bien marquées, soit de taille plus réduite et de forme quadrangulaire. Le faciès patiné de ce silex est gris-blanc à bleuté, opaque, et présente des vermicules blanchâtres très caractéristiques. Le second est un silex opaque, gris clair à brun-beige, présentant quelques plages translucides (fig. $46, n^{\circ} 4$ ). Les grains sont fins, mais un réseau de cément translucide donne un aspect granuleux au silex. La roche d'origine devait être un calcaire du type mudstone, qui est maintenant entièrement silicifié. Sous binoculaire, on observe de nombreuses oogones de charophytes. Le cortex est assez épais (de l'ordre du centimètre), blanc ou ocre. Le faciès patiné a une teinte blanche à crème ; quelques pièces montrent une légère zonation. Le cortex apparaît plus jaunâtre et peut être sensiblement plus épais.

Le silex secondaire est généralement un silex noir à gris, translucide, à grains très fins, de bonne qualité

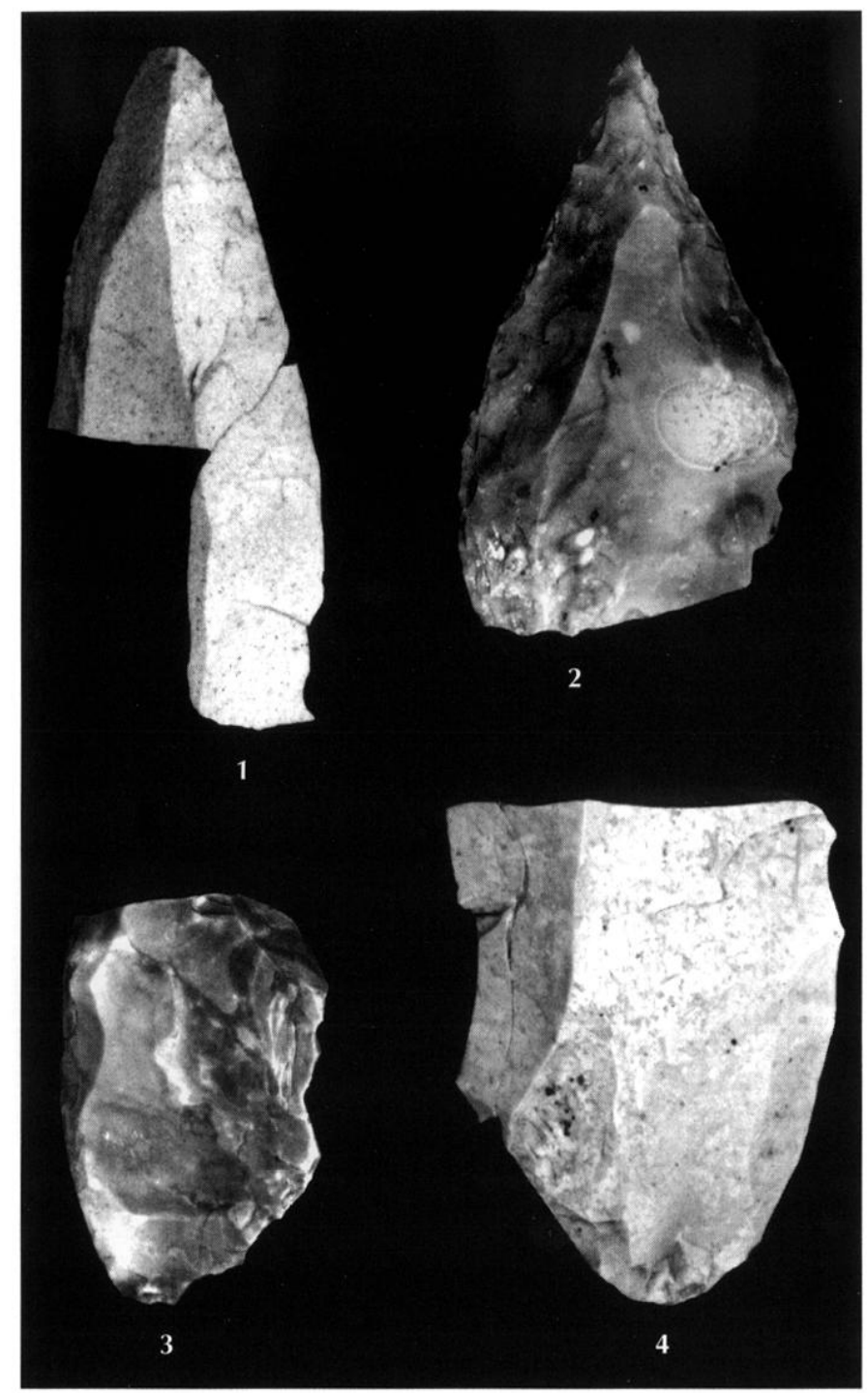

Fig. 46 - Villiers-Adam, Le Petit Saule : vue des différentes matières premières utilisées par les hommes préhistoriques. 1 , grès ; 2 , silex secondaire; 3, silex tertiaire translucide; 4, silex tertiaire opaque (photo J.-Y. Lacote, Archives départementales du Val-d'Oise).

(tig. 46, $\mathrm{n}^{\circ}$ 2). Des taches nucléées plus claires sont souvent observables sans qu'il y ait de zonations et on observe dans quelques pièces des inclusions filamenteuses de couleur rougeâtre ou blanchâtre. Les cortex sont légèrement granuleux, très fins. Ils dépassent rarement plus de $2 \mathrm{~mm}$ d'épaisseur. Certains cortex sont verdis, peut-être en raison de la présence de glauconie ou d'un remaniement d'origine marine au Thanétien, et d'autres présentent une altération pédogénétique continentale que l'on retrouve dans les formations d'argile à silex. Les blocs sont de taille plus petite que ceux du silex 
tertiaire et ont une forme branchue, les surfaces corticales y étant donc plus importantes. Le faciès patiné est opaque, gris à blanc, parfois légèrement bleuté. L'épaisseur de la patine peut atteindre un millimètre d'épaisseur. Le cortex prend alors une teinte jaune à brune.

Deux types de grès ont été utilisés par les Paléolithiques. Le premier est de couleur gris à blanc-bleu. C'est un grès quartzite à grains moyens très bien silicifié, compact (fig. $46, \mathrm{n}^{\circ} 1$ ). Les blocs sont généralement épais d'une quinzaine de centimètres, tabulaires au sommet et bulbeux à la base. Le cortex est brun à brunjaune et peu épais. Le second, moins bien silicifié, correspond dans le détail à un faciès identique. Il a une teinte jaunâtre et présente parfois des litages diffus. Moins compact que l'autre faciès, il reste une matière première utilisable.

Un matériau de piètre qualité, circonscrit au niveau de l'amas 3 , est un calcaire très bioclastique qui présente de nombreux restes d'invertébrés fossiles, dont Potamides, qui indique une influence de dessalure, mais sans milioles, le tout enrobé dans une matrice sableuse très silicifiée. D'après D. Obert, cette roche correspond à un faciès local situé au sommet des sables de Beauchamp ou à la base des calcaires de Saint-Ouen. Ces faciès ont été oxydés et sont les témoins d'un paléosol post-auversien. Dans les décomptes, ce matériau a été assimilé au silex tertiaire.

Le silex tertiaire représente $86,90 \%$ du matériel du niveau principal N2 ; le silex secondaire, $11,25 \%$; le grès conglomératique, $5,37 \%$ et le grès, seulement $1,85 \%$.

\section{ZONES D'APPROVISIONNEMENT POSSIBLES}

Les Paléolithiques de Villiers-Adam ont pu recueillir les matières premières qu'ils ont ensuite utilisées à différentes sources, plus ou moins éloignées du site.

Le silex tertiaire est d'origine locale. Il provient du calcaire de Saint-Ouen, qui affleure quelques dizaines de mètres à l'est, au nord et au sud du gisement. D'âge bartonien supérieur (Marinésien), le calcaire de SaintOuen est une formation laguno-continentale qui forme l'assise de la Plaine de France. D'une dizaine de mètres d'épaisseur, il est constitué de calcaires sublithographiques durs ou marneux tendres, de marnes et de gypse (Cavelier et al., 1980). Les accidents siliceux y sont fréquents, leur faciès pouvant varier au sein d'un même banc. Une seconde source d'approvisionnement possible est le cailloutis plio-quaternaire qui recouvre les sables et grès de Beauchamp (unité 22). Le matériau y est sans doute de moins bonne qualité, mais il présentait l'avantage d'être disponible sur place lors de l'occupation paléolithique, puisque le cailloutis affleurait au niveau du secteur 2. Il est probable que les blocs issus de ce cailloutis présentaient un degré de gélifraction plus important que des rognons en position primaire ou secondaire de proximité.

Le grès est également d'origine locale, puisque les sables et grès de Beauchamp forment le substrat du gisement. Cette formation marine, d'âge bartonien inférieur (Auversien), est constituée de sables blancs, fins et bien classés, à stratifications planes, et présente à son sommet plusieurs barres gréseuses dont la compacité et la silicification diminuent vers le bas. Ces dernières sont l'expression d'une émersion et d'une pédogenèse de type podzolique post-auversienne (Cavelier et al., 1980). Plusieurs dalles de grès affleurent en particulier à l'ouest du secteur 3, en direction du Chamesson. Le calcaire bioclastique silicifié de l'amas 3 est également d'origine locale.

Par contre, le silex crétacé est obligatoirement allochtone. Il ne peut provenir ni des formations tertiaires locales ni du cailloutis plio-quaternaire, fruit du remaniement des niveaux bartoniens et stampiens. Trois sources principales d'origine de cette matière première peuvent être envisagées: les formations crayeuses à proprement parler, leur remaniement plio-quaternaire (argiles à silex et poches karstiques associées) et les formations fluviatiles de la vallée de l'Oise. Les affleurements en position primaire les plus proches sont situés dans la région de Champagne-sur-Oise, au nord de L'IsleAdam et de Presles, à environ $5 \mathrm{~km}$ au nord du site. Il s'agit de niveaux d'âge campanien, formés de craie blanche à lits réguliers de rognons de silex, qui constituent vers le nord-ouest le substrat du pays de Thelle et de l'anticlinal de Bray et, plus vers l'est, de la bordure septentrionale du Parisis. Le remaniement de ces formations crayeuses a entraîné la mise en place d'argiles à silex - très développées dans le nord-ouest du pays de Thelle et contenant des silex branchus peu brisés, brunis en surface et de sables à silex dans le Parisis septentrional - contenant des silex brisés à patine blanche. Les affleurements de ces formations secondaires sont proches des affleurements crétacés et sont donc éloignés du gisement 


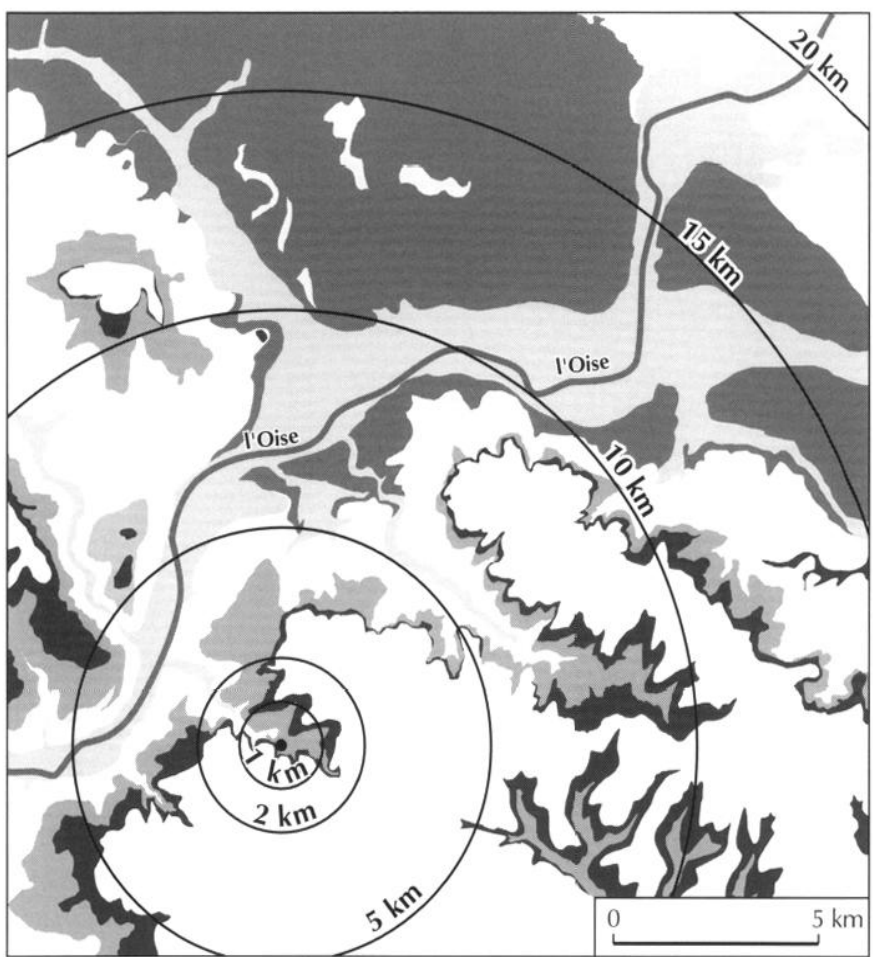

- site du Petit Saule à Villiers-Adam alluvions et terrasses fluviatiles sables de Beauchamp (Bartonien inférieur)

Fig. 47 - Origine possible des différentes matières premières (dessin J.-J. Bahain).

paléolithique de Villiers-Adam d'environ $5 \mathrm{~km}$ (fig. 47). Les formations crétacées ont été remaniées sur place dans l'argile à silex et piégées dans des poches karstiques avec le Thanétien résiduel et, par conséquent, avec le silex à cortex verdi.

Dernière source d'approvisionnement possible, la vallée de l'Oise est distante du site d'environ $3 \mathrm{~km}$ à l'ouest. Il faut toutefois souligner que l'aspect très frais du cortex des silex secondaires, l'absence de lustrage et de cupules de choc, fréquents sur les rognons issus d'alluvions, et le fait que des plaquages crayeux soient conservés sur quelques artefacts tendent à écarter l'hypothèse de l'utilisation de cette source de matière première.

La stratégie d'acquisition de la matière première semble donc avoir été orientée vers deux pôles :

- le silex tertiaire, d'origine bartonienne;

- le silex campanien remanié dans l'argile à silex (y compris le thanétien à cortex verdi).

\section{ÉTUDES TECHNIQUES ET TYPOLOGIQUES}

\section{MÉTHOde D'ÉTUDE}

L'approche des industries lithiques du gisement de Villiers-Adam repose sur l'étude globale des séries, grâce à la lecture technologique de l'ensemble du matériel (nucléus, produits), complétée par l'analyse des remontages. Les chaînes opératoires, Levallois et non-Levallois, sont décrites grâce à l'identification des schémas opératoires, complétée par l'étude de l'outillage retouché. L'attribution typologique de certains produits de débitage est parfois remise en cause par les résultats des remontages. L'étude technique présentée ici fait appel à une terminologie et à une séric de définitions et de notions qu'il paraît necéssaire de préciser dans un souci de clarté (Révillion, 1994).

\section{Le débitage Levallois}

La question de la reconnaissance et de l'identification des méthodes de débitage Levallois a été largement débattue ces dernières années (Boëda, 1994 ; Van Pecr, 1992 ; Dibble, Bar-Yosef, 1995). En fonction de la conception volumétrique et de la morphologie constante (Boëda, 1997) qui régissent l'exploitation des nucléus Levallois, grâce à la préparation et à l'entretien des convexités d'une surface productive, on distingue deux modalités : le débitage Levallois stricto sensu, permettant la production d'un éclat préférentiel par surface de débitage, et le débitage récurrent, permettant la production successive de plusieurs éclats de type Levallois, par le biais de plusieurs schémas opératoires (unipolaire, bipolaire ou centripète).

\section{Le débitage non-Levallois}

À la différence du débitage L evallois, le débitage nonLevallois est essentiellement caractérisé par des concepts d'exploitation différents qui s'expriment aux dépens d'une ou de plusieurs surfaces productives et par l'absence de phases de préparation et d'entretien des convexités de ces surfaces. Ces dernières ne sont pas forcément hiérachisées. La production est généralement conduite grâce aux convexités naturelles des blocs de matières premières. Dans certains cas, des remontages démontrent que le débitage non-Levallois conduit à la 
production d'éclats pouvant être considérés comme typologiquement Levallois en raison de leurs caractéristiques morphologiques.

Parmi ces chaînes opératoires non-Levallois, le débitage laminaire, au sein des industries du Paléolithique moyen en Europe septentrionale, fait l'objet, depuis plusieurs années, d'études techno-typologiques qui ont permis de mettre en évidence et de préciser un certain nombre de concepts régissant les différents schémas opératoires laminaires (Révillion, 1994 ; Locht dir, 2002).

\section{CARACTÉRISTIQUeS TECHNIQUeS,}

\section{MÉTHODE DE PERCUSSION ET MATÉRIAUX}

Dans le secteur 1, le silex tertiaire est celui qui a été le plus largement exploité $(84,92 \%)$. Le silex secondaire $(7,32 \%)$ et le grès $(1,88 \%)$ ont été débités en quantité assez réduite; un seul bloc de calcaire bioclastique silicifié a été exploité $(5,88 \%)$.

Dans le secteur 2, le silex secondaire est majoritaire $(65,31 \%)$ par rapport au silex tertiaire $(33,33 \%)$. Le grès y a été très peu exploité $(1,36 \%)$.

Dans le secteur 3, ces proportions s'inversent. Le silex tertiaire y représente $76,36 \%$ de la série, le silex crétacé, $21,82 \%$ et le grès, seulement $1,82 \%$.

Dans les trois zones, la taille du grès et du silex tertiaire a eu lieu sur place, comme l'atteste la présence de déchets de taille provenant de toutes les étapes des différentes chaînes opératoires. Cela est confirmé par les nombreux remontages effectués sur les blocs de ces deux matériaux. En ce qui concerne le silex crétacé, les choses sont moins claires, car les éclats corticaux sont peu représentés. De plus, aucun des remontages effectués n'a permis de reconstituer la forme d'origine de l'un de ces blocs. Ces arguments permettent de penser qu'il y a eu un fractionnement dans l'espace des activités de taille, tout au moins pour ce dernier matériau.

L'usage de la percussion directe au percuteur dur est attesté. Les talons lisses sont largement majoritaires (tabl. XI).

\section{ÉCONOMIE DES MATIÈRES PREMIÈRES}

Le silex tertiaire, d'origine locale, représente $86,90 \%$ du matériel du niveau principal. Il s'agit d'un matériau de qualité variable selon les blocs $(87,242 \mathrm{~kg})$ (tabl. XII).
Tabl. XI - Décompte des différents types de talons par secteur au Petit Saule à Villiers-Adam.

\begin{tabular}{|l|c|c|c|c|}
\hline \multicolumn{1}{|c|}{ Types de talons } & Secteur $\mathbf{1}$ & Secteur $\mathbf{2}$ & Secteur $\mathbf{3}$ & Total \\
\hline Talons corticaux & 80 & 8 & 1 & 89 \\
\hline Talons lisses & 668 & 38 & 20 & 726 \\
\hline Talons punctiformes & 89 & 14 & 6 & 109 \\
\hline Talons dièdres & 113 & 6 & 2 & 121 \\
\hline Talons facettés & 168 & 23 & 5 & 196 \\
\hline Chapeau de gendarme & 5 & 1 & 0 & 6 \\
\hline Talons ôtés & 6 & 1 & 0 & 7 \\
\hline Total & $\mathbf{1 1 2 9}$ & $\mathbf{9 1}$ & $\mathbf{3 4}$ & $\mathbf{1 2 5 4}$ \\
\hline
\end{tabular}

Tabl. XII - Poids des différents matériaux par secteur au Petit Saule à Villiers-Adam.

\begin{tabular}{|c|c|c|c|c|}
\hline & $\begin{array}{c}\text { Silex } \\
\text { tertiaire }(\mathbf{k g})\end{array}$ & $\begin{array}{c}\text { Silex } \\
\text { secondaire }(\mathbf{k g})\end{array}$ & $\begin{array}{c}\text { Grès } \\
\mathbf{( k g})\end{array}$ & $\begin{array}{c}\text { Total } \\
(\mathbf{k g})\end{array}$ \\
\hline Secteur $\mathbf{1}$ & 85,108 & 4,974 & 2,904 & $\mathbf{9 2 , 9 8 6}$ \\
\hline Secteur $\mathbf{2}$ & 1,06 & 1,478 & 0,036 & $\mathbf{2 , 5 7 4}$ \\
\hline Secteur 3 & 1,074 & 0,242 & 0,075 & $\mathbf{1 , 3 9 1}$ \\
\hline Total & $\mathbf{8 7 , 2 4 2}$ & $\mathbf{6 , 6 9 4}$ & $\mathbf{3 , 0 1 5}$ & $\mathbf{9 6 , 9 5 1}$ \\
\hline
\end{tabular}

Il a été exploité de façon peu économe par les tailleurs. Plusieurs blocs ont fait l'objet d'un simple test. Les accidents de taille sont fréquents, ainsi que les cassons qui en résultent.

Le silex crétacé présent sur le gisement représente un poids de $6,694 \mathrm{~kg}$ seulement (tabl. XII). Le transport d'une telle masse pondérale par les chasseurs nomades du Paléolithique moyen sur quelques kilomètres est envisageable car il s'agit d'un matériau de très bonne qualité. Tous les blocs de silex crétacé ont été exploités ; aucun n'est resté à l'état de bloc testé. D'après le nombre de nucléus, l'hypothèse du transport d'une vingtaine de blocs de dimensions modestes peut être proposée. La mise en évidence du prélèvement sélectif d'un matériau sur un gîte d'approvisionnement éloigné du site est un fait remarquable pour une occupation de plein air. En effet, en règle générale, dans les gisements de plein air du nord-ouest de la France, les modalités d'approvisionnement en silex ne peuvent être appréhendées, car le silex crétacé est abondant et les apports lœssiques du Weichsélien ont souvent colmaté les paléoreliefs (Ameloot-Van der Heijden, 1996).

Le grès, d'origine locale, n'a été exploité que de façon anecdotique $(3,015 \mathrm{~kg})$, bien que les artefacts 
réalisés sur ce matériau soient parfois de belle facture (tabl. XII).

\section{LA SÉRIE DU SECTEUR 1}

Cet ensemble contient 2190 artefacts, qui proviennent de chacune des étapes du débitage (tabl. XIII). 827 d'entre eux, soit $37,76 \%$, étaient concentrés dans les postes de débitage (fig. 42). De nombreux remontages ont été effectués sur ce matériel $(21,14 \%$ de la totalité des artefacts, 22,03\% si l'on ne tient pas compte des esquilles). Les raccords de cassures anciennes sont beaucoup moins nombreux, puisque seulement $3,31 \%$ des artefacts ont été recollés (3,45\% sans les esquilles).

\section{Chaîne opératoire à pointes}

La production de pointes s'est effectuée par le biais d'un débitage unipolaire convergent. Cette chaîne opératoire est représentée par 84 nucléus, soit $49,7 \%$ de la totalité de cette population. Le choix des blocs porte sur des volumes quadrangulaires possédant des arêtes naturelles. Le tailleur profite des convexités originelles des blocs, avec une préparation sommaire des plans de frappe.

L'exploitation de la surface productive commence par des enlèvements laminaires corticaux de section triangulaire, parfois très épais, de directions convergentes. Ils ont pour but de créer des arêtes qui permettront l'obtention de pointes unipolaires. Ils sont détachés dans l'axe de la plus grande longueur du nucléus. Cette séquence de base, ou algorithme (Boëda, 1997), peut être répétée plusieurs fois de suite sur la même surface productive.

Soixante-six nucléus unipolaires convergents ont été réalisés sur blocs de silex tertiaire de qualité variable. Ce mème schéma opèratoire a èté reproduit sur dix éclats. Seulement cinq nucléus de ce type sont en silex secondaire. En raison de la qualité de cette dernière matière première, les blocs ont été exploités jusqu'à la limite des convexités de la surface productive. Le grès a été également exploité par le biais de ce schéma. Trois nucléus, dont un réalisé sur un éclat épais, ont permis le détachement de quelques enlèvements à partir d'un plan de frappe sommairement préparé.

Cette chaîne opératoire est présente sous différentes modalités, qui obéissent à des règles techniques iden-
Tabl. XIII - Décompte de la série N2 du secteur 1 du Petit Saule à Villiers-Adam.

\begin{tabular}{|l|r|r|}
\hline & Nombre & \multicolumn{1}{c|}{$\%$} \\
\hline Éclats corticaux & 642 & 29,52 \\
\hline Éclats & 647 & 29,76 \\
\hline Pointes & 56 & 2,57 \\
\hline Pointes pseudo-Levallois & 21 & 0,97 \\
\hline Lames & 163 & 7,49 \\
\hline Éclats Levallois & 83 & 3,81 \\
\hline Éclats débordant & 19 & 0,87 \\
\hline Esquilles & 89 & 4,09 \\
\hline Cassons & 269 & 12,37 \\
\hline Nucléus & 170 & 7,81 \\
\hline Blocs testés & 16 & 0,74 \\
\hline Total & $\mathbf{2 1 9 0}$ & $\mathbf{1 0 0 , 0 0}$ \\
\hline
\end{tabular}

tiques, et dont l'identification a été permise par les remontages effectués au sein de cette série. L'application de ces modalités est tributaire de la forme d'origine du bloc. Le degré d'exploitation varie en fonction de la morphologie du bloc et de la qualité de la matière.

Ces nucléus présentent le plus souvent une seule surface de production. Cependant, les remontages ont démontré que plusieurs surfaces de débitage pouvaient s'inscrire de façon indépendante dans le volume du bloc d'origine. Elles s'articulent selon des plans qui peuvent être parallèles ou perpendiculaires (fig. 50 et 56). La production se déroule alors à partir de surfaces exploitées de façon alternative. Ces observations évoquent celles qui ont pu être réalisées pour le Clactonien quant à la gestion des nucléus, sur lesquels les surfaces productives peuvent être localisées sur une autre partie du bloc, sans contact avec la précédente (Forestier, 1993 ; Boëda, 1997). L'objectif du débitage à Villiers-Adam reste toutefois l'obtention de pointes typologiquement Levallois.

\section{Modalité unipolaire, convergente simple sur une surface (62 nucléus)}

Cette modalité a été appliquée sur de petits blocs ou sur des éclats volumineux souvent corticaux. Après avoir installé un plan de frappe sur l'une des extrémités du bloc, un produit à dos cortical est enlevé sur chacun des côtés. Les deux enlèvements convergent, ce qui met en place des conditions favorables (convexités, nervures) pour le détachement d'une pointe typologiquement 

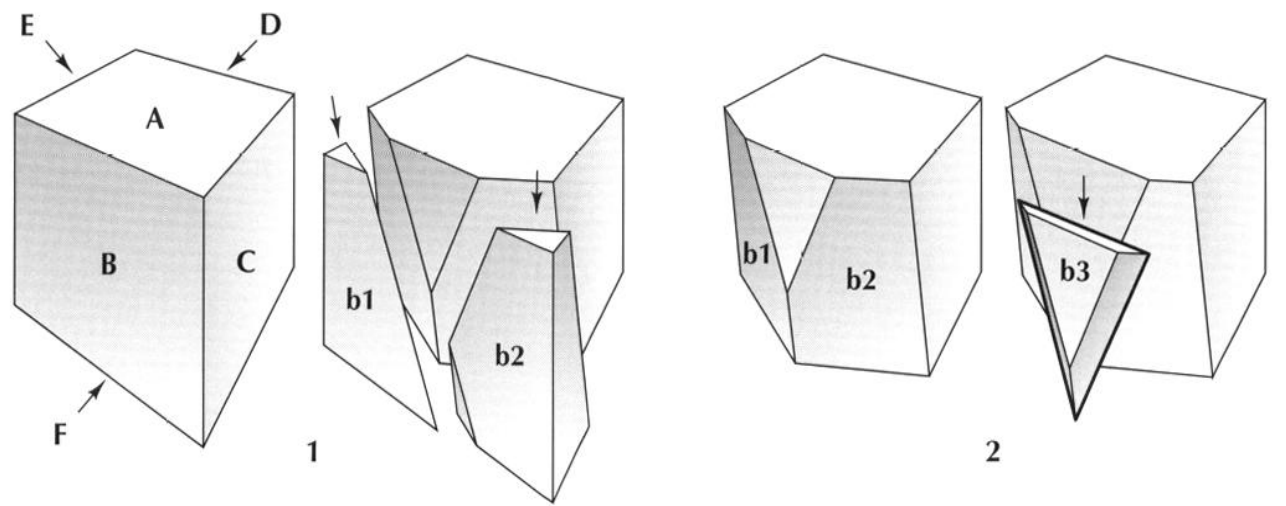

2

Fig. 48 - Débitage unipolaire convergent simple sur une surface (dessin P. Rajmond).

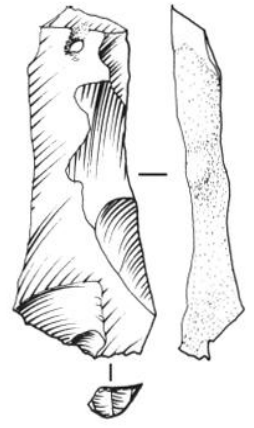

1

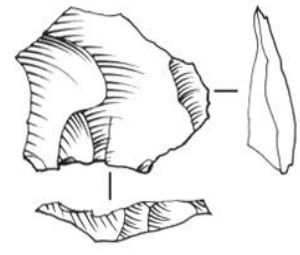

2
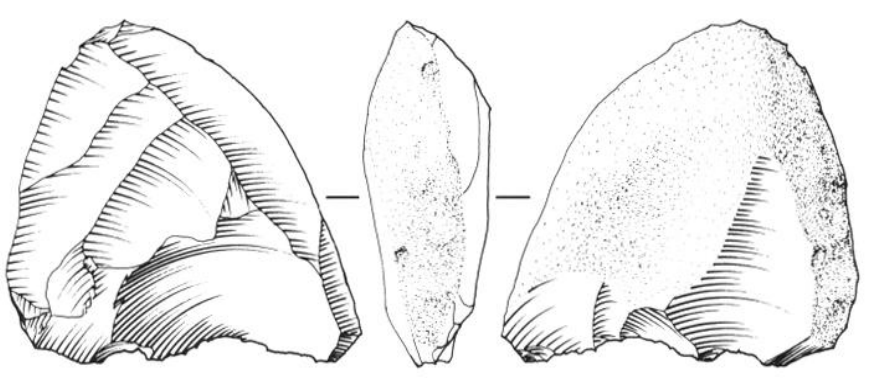

3
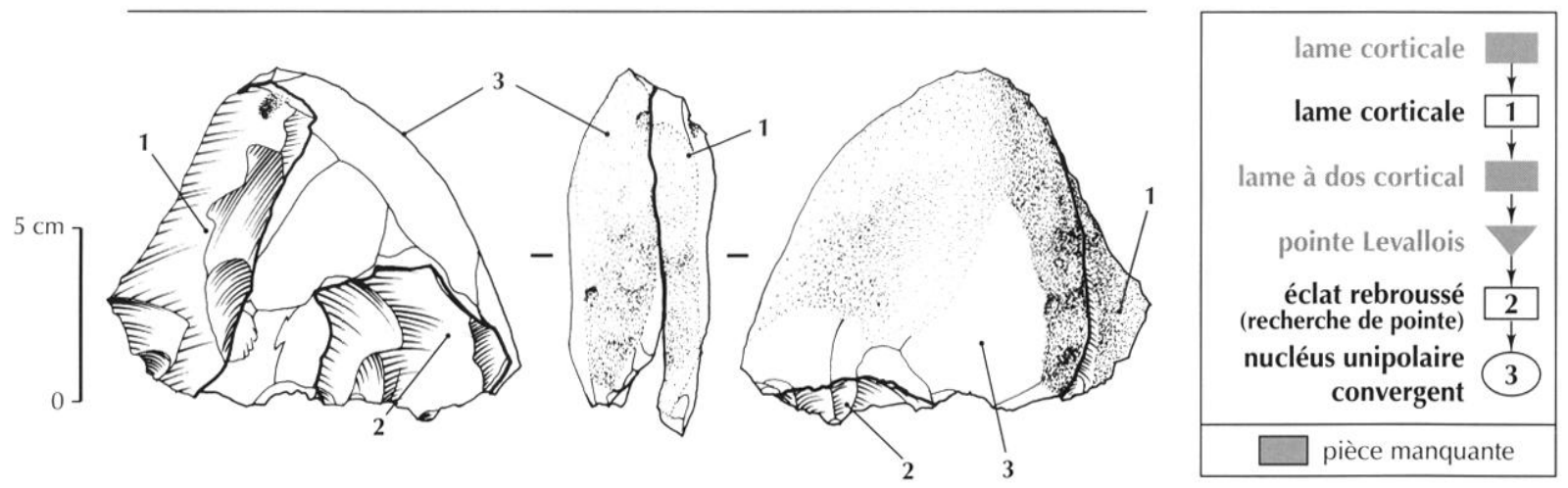

Fig. 49 - Remontage 11 (dessin P. Raymond).

Levallois. Cette séquence de base, composée de deux produits à dos et d'une pointe, est soit unique (fig. 48, $\mathrm{n}^{\text {\%s }} 1$ et 2), soit répétée plusieurs fois, en fonction du principe de récurrence, avec ou sans réaménagement du plan de frappe.

Le remontage de l'ensemble 11 illustre ce schéma de production (fig. 49). Le choix du tailleur s'est porté sur un petit rognon de silex secondaire de bonne qualité. Après une ouverture du plan de frappe par plusieurs enlè- vements parallèles sur l'une des extrémités du bloc, deux lames à dos cortical, dont l'une a été remontée sur le nucléus, ont été détachées sur chacun des côtés de la surface productive. Ces gestes techniques ont mis en place les critères permettant le détachement d'une pointe (convexités, nervure-guide,...). D'après son négatif, la pointe obtenue était de forme régulière. Elle n'a pas été retrouvée. La tentative d'obtention d'une deuxième pointe, sans repréparation des convexités, a échoué. Le 


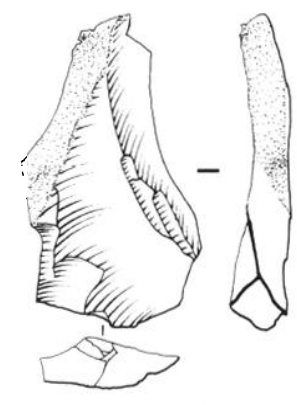

1

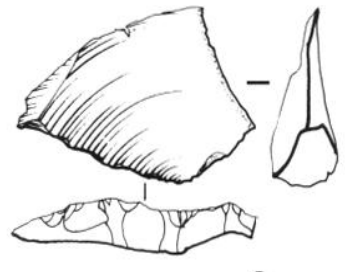

2

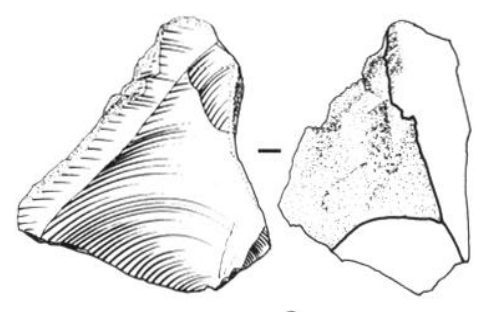

3

Fig. 50 - Remontage 39. Les numéros indiqués sous les artefacts lithiques renvoient ci leur position dans le schéma diacritique (dessin P. Raymond).
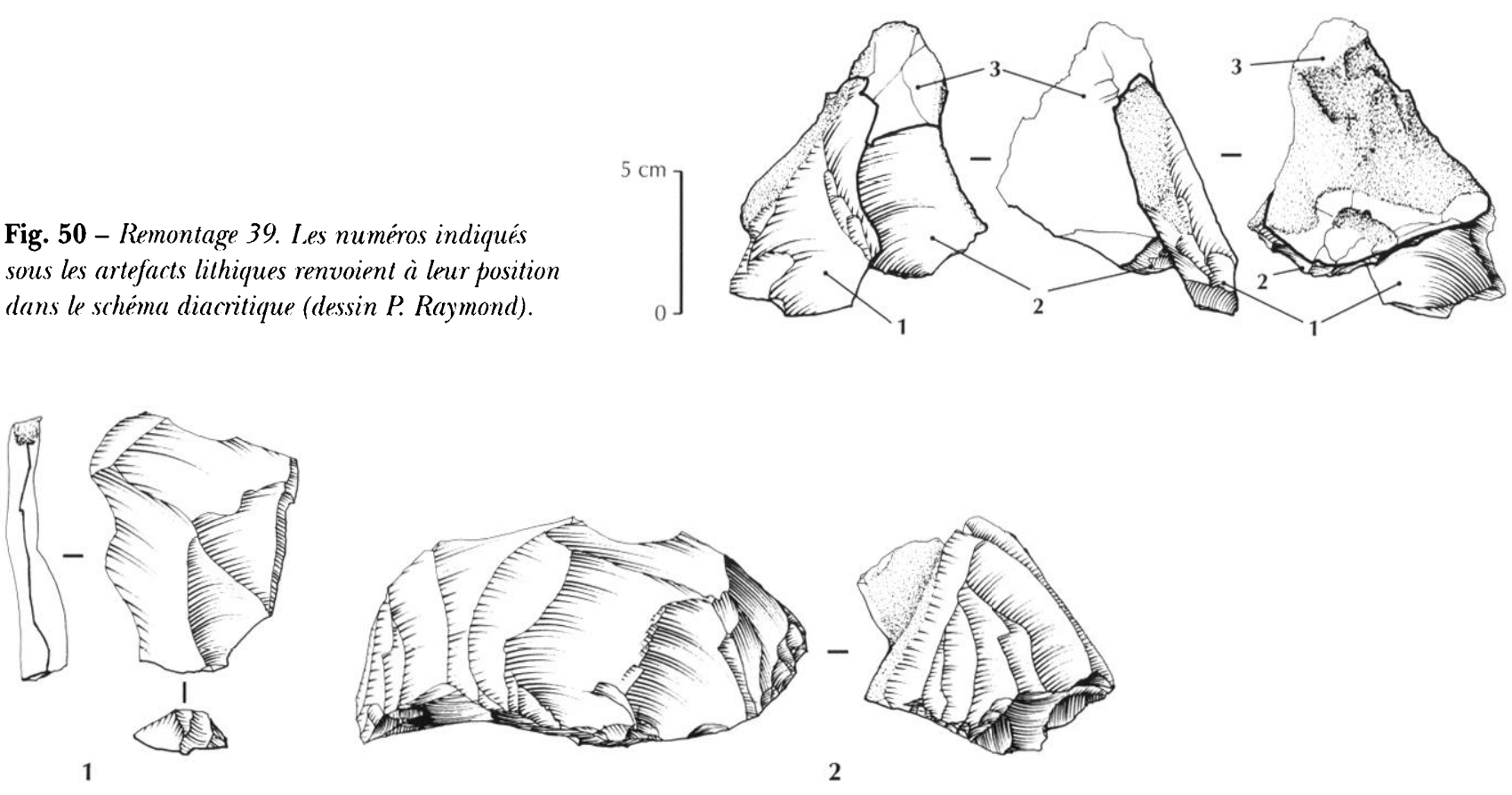

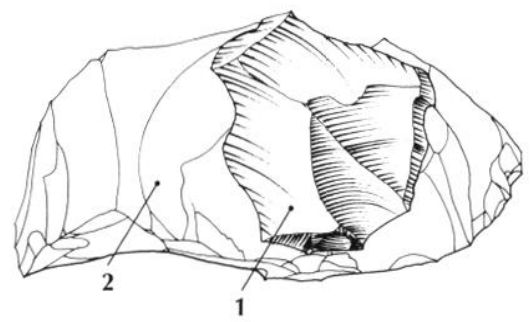

produit détaché est un éclat court et rebroussé. Le débitage est arrêté après cette dernière étape.

Sur plusieurs autres remontages de ce type, des lames à dos cortical ont été remontées sur le nucléus, sans que cela soit possible pour la pointe (fig. 50 , ensemble 39 ).

Dans certains cas, le plan de frappe est installé sur le côté le plus long du bloc. L'exploitation du bloc se fait dans l'axe de la largeur. Les éclats détachés dans cette direction outrepassent fréquemment. Après l'obtention
Fig. 51 - Ensemble 25 : remontage d'un éclat typologiquement Levallois (1) sur un nucléus unipolaire convergent plus large que long (2) (dessin P. Raymond). d'une première série d'éclats, l'algorithme de base peut être décalé sur l'un des côtés de la surface productive, et la production se fait sur le côté du nucléus, sur la même surface, mais dans un plan légèrement sécant par rapport au premier (fig. 51, ensemble 25). Les produits obtenus sont assez courts et peuvent être typologiquement Levallois.

La fragmentation des blocs au cours du débitage est fréquente. La plupart du temps, les différents morceaux 

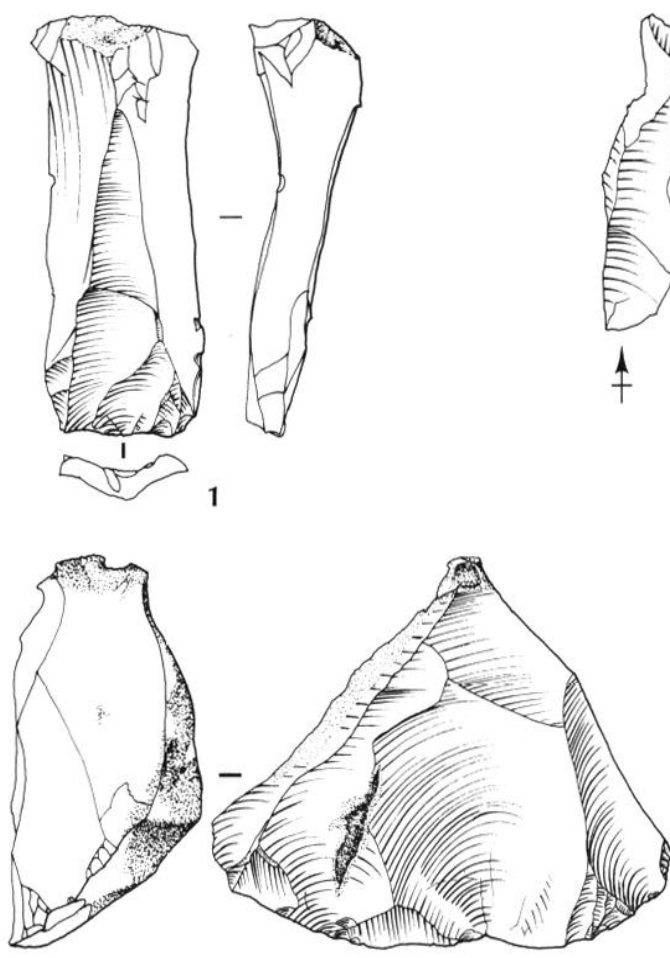

4
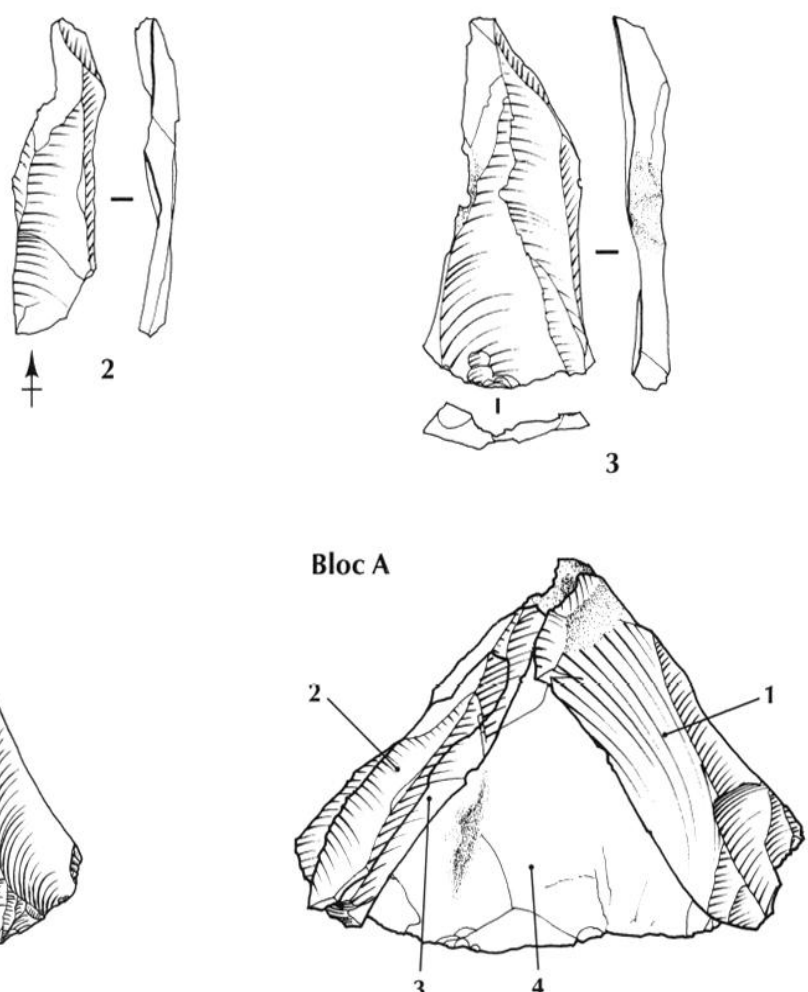
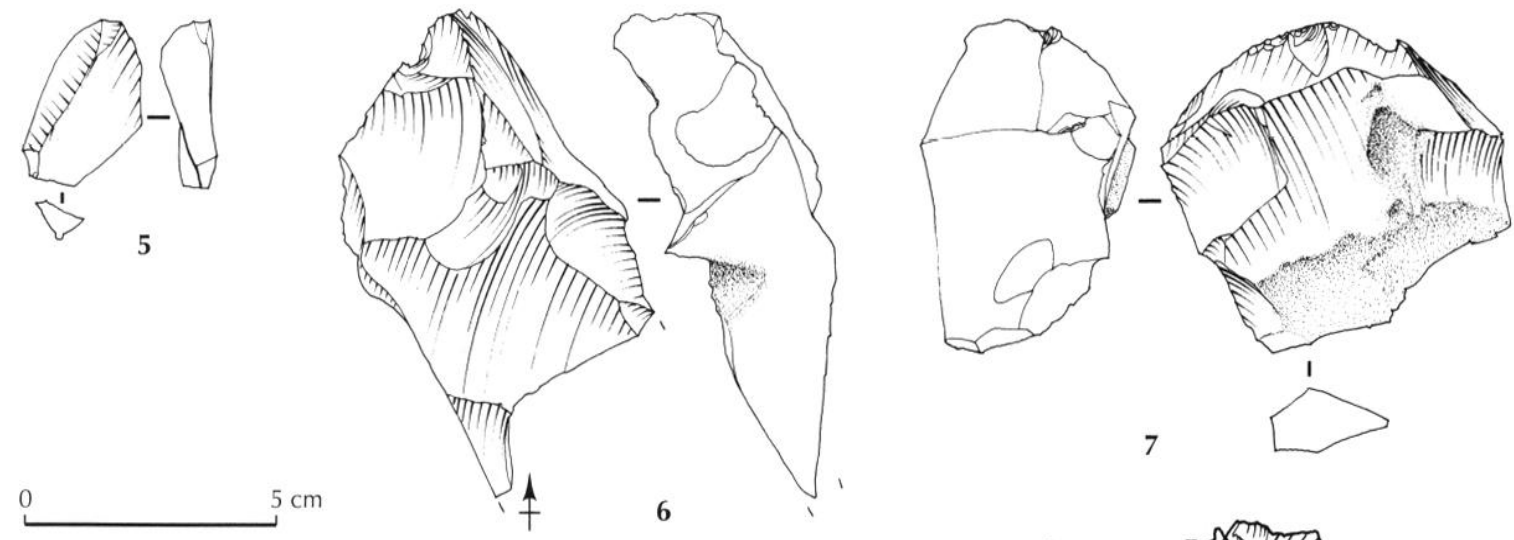

0 $5 \mathrm{~cm}$
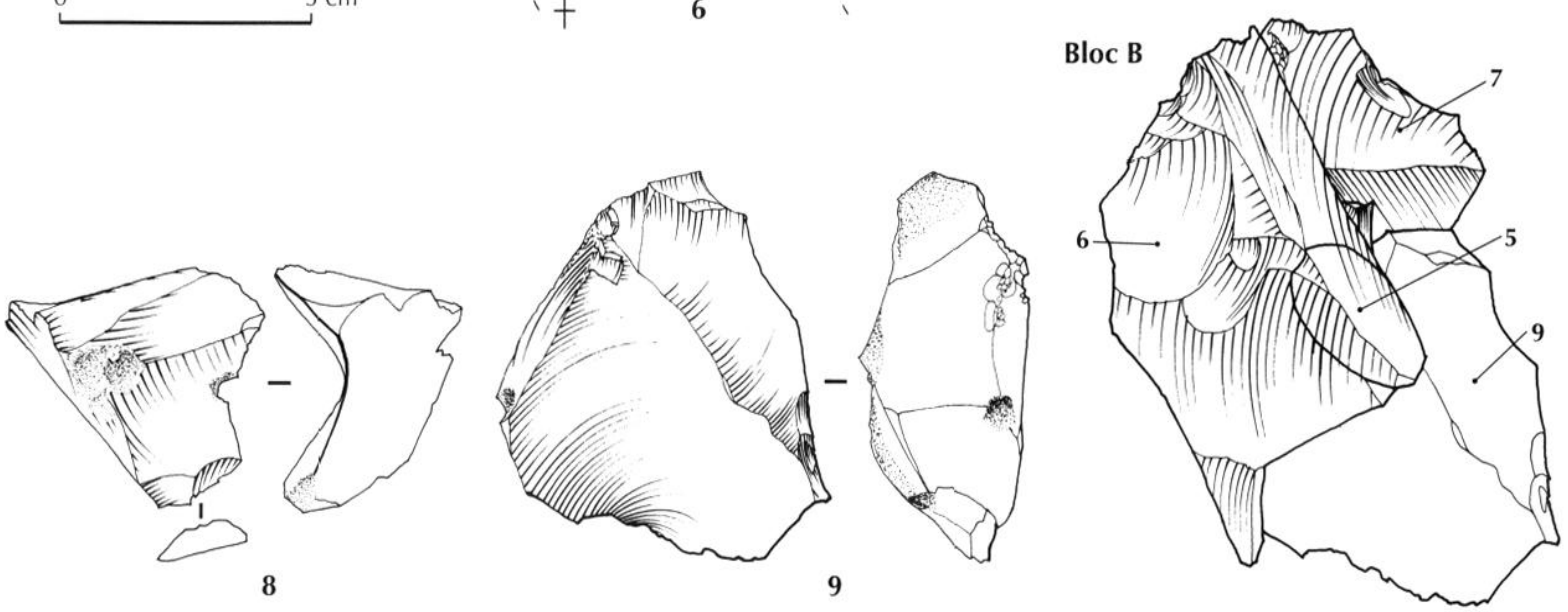

Fig. 52 - Remontages des blocs A et B de l'ensemble 34 (dessin P. Raymond). 


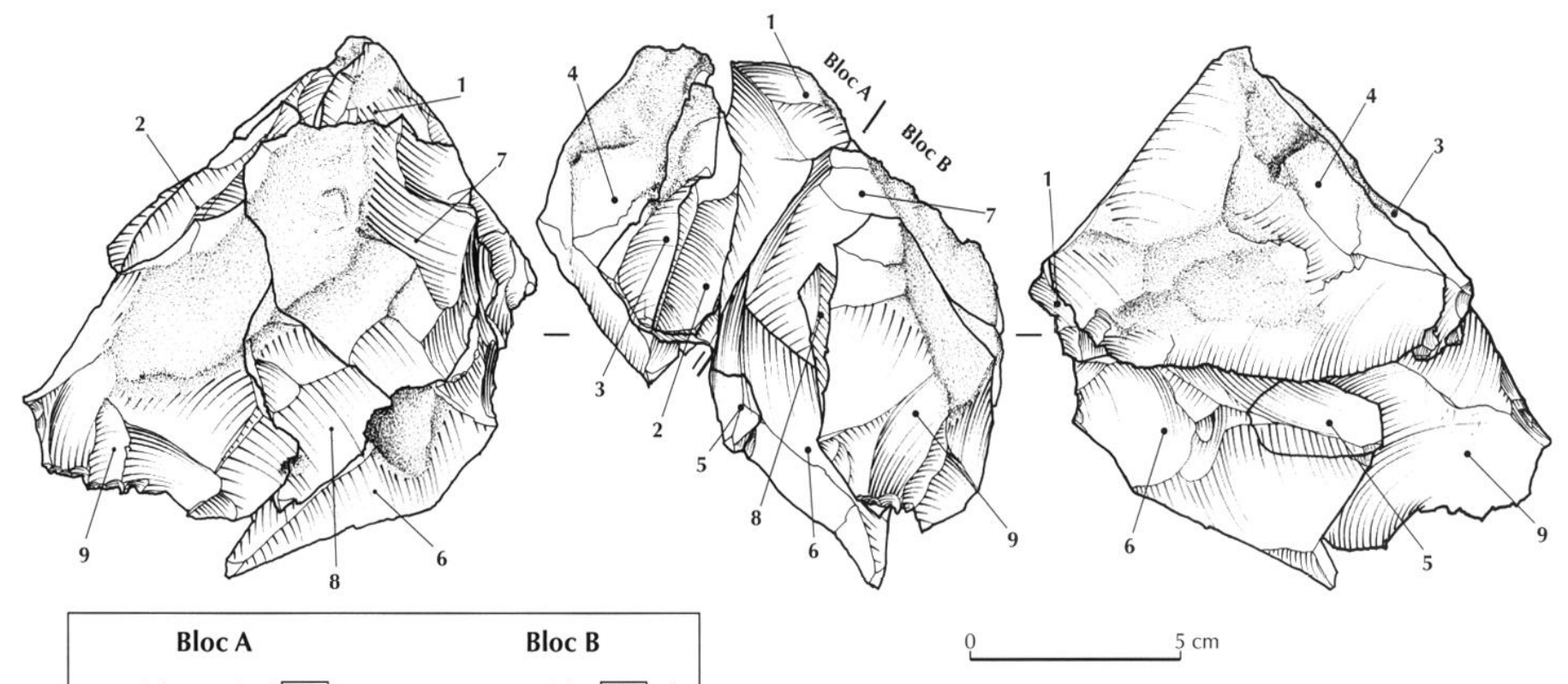

dont un a outrepassé, d'un éclat de préparation de plan de frappe (fig. $52, n^{\circ} 7$ ) et d'un éclat (fig. $52, n^{\circ} 5$ ), enlevé sur la face supérieure de l'éclat débordant après son détachement du nucléus. Le tailleur installe un premier plan de frappe par au moins quatre enlèvements et détache une série de deux éclats courts, réfléchis. Après cet accident, un deuxième plan de frappe est installé sur le côté opposé du bloc. Le tailleur a enlevé un épais éclat débordant (fig. 52, $n^{\circ} 6$ ). Puis il est revenu sur le premier plan de frappe pour y détacher un éclat de repréparation du plan de frappe (fig. 52, $\mathrm{n}^{\circ} 7$ ) suivi d'une série de trois enlèvements productifs non remontés. Il a détaché un nouvel éclat de repréparation du plan de frappe (fig. 52, $n^{\circ} 8$ et fig. 53) qui a réfléchi. Ce dernier a été suivi d'une pointe débitée à partir du plan de frappe opposé. Un éclat laminaire débordant est ensuite enlevé à partir d'un plan de frappe opposé et sommairement aménagé, peut-être pour ôter une arête gênant la poursuite du débitage, qui s'arrête toutefois après cet enlèvement. Ce geste technique n'a été réalisé que sur 3 nucléus de cette série. Toutefois, il ne s'agit pas d'un schéma opératoire différent mais d'une légère variante du débitage unipolaire afin de contourner un obstacle technique.

Modalité unipolaire, convergente à deux surfaces productives débitées successivement (22 nucléus)

Dans ce cas de figure, les deux surfaces sont exploitées successivement. Plusieurs séquences de base (fig. 52, $n^{\circ} 9$ ), de deux éclats débordants (fig. 52, $n^{\circ} 6$ ) 

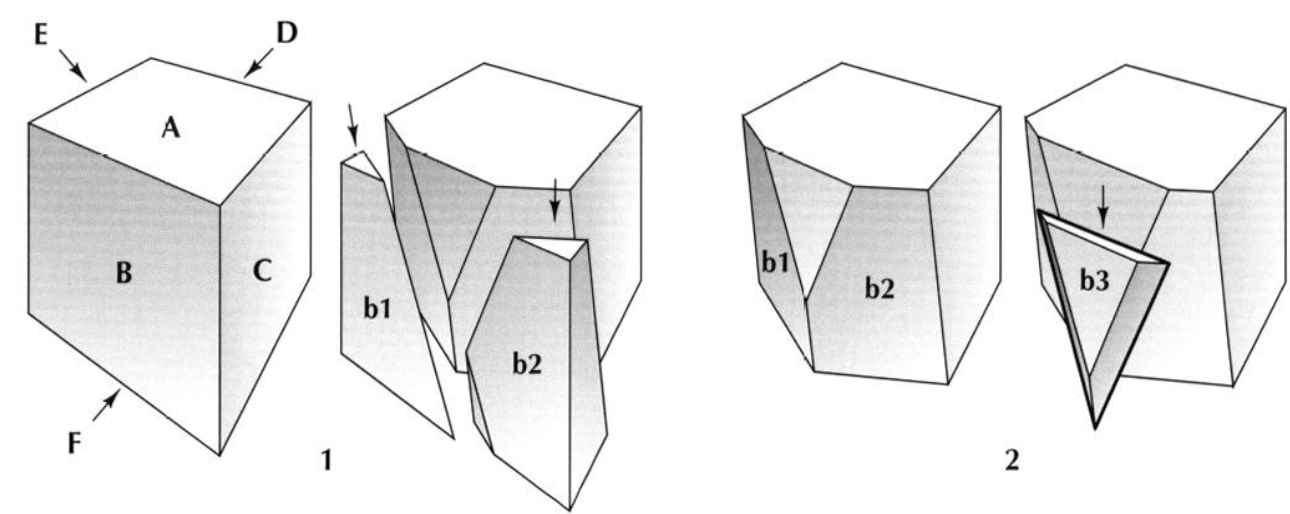

2
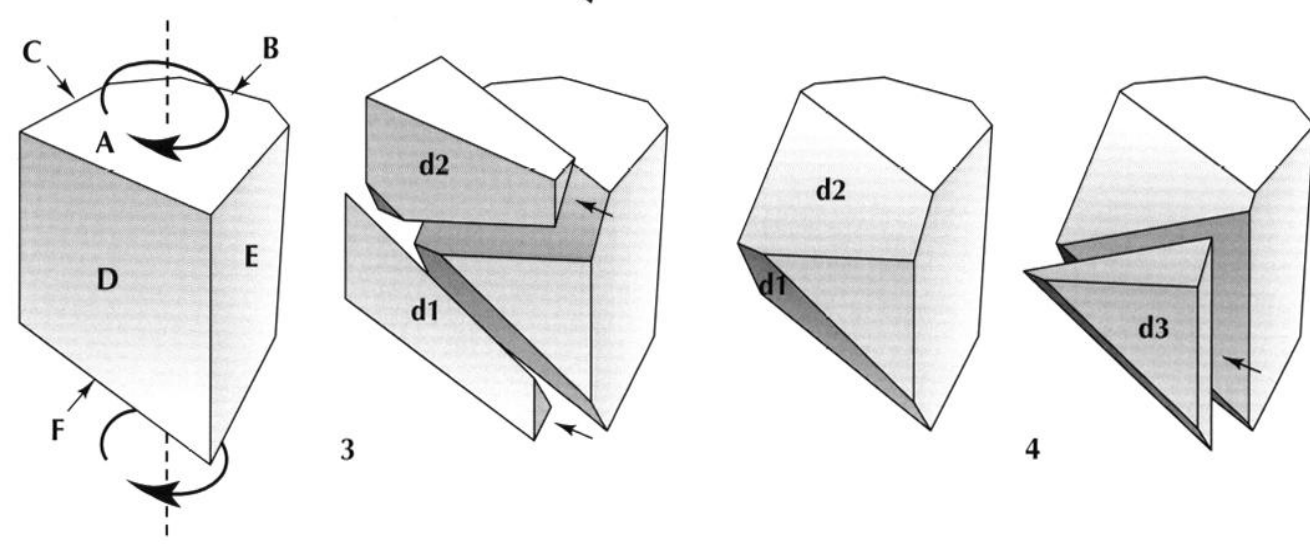

Fig. 54 - Débitage unipolaire convergent : schéma d'un bloc à deux surfaces s'inscrivant dans des plans paralleles (dessin P. Raymond).

(algorithmes) peuvent s'enchaîner sur une même surface productive. Les deux surfaces peuvent s'inscrire de deux façons dans le volume du bloc. Le nombre de surfaces par nucléus est limité à deux dans cette série.

Dans le premier cas, les deux surfaces s'inscrivent dans des plans parallèles et les directions des enlèvements sont perpendiculaires (fig. 54). Ce schéma opératoire a été mis en évidence par le remontage de l'ensemble 8 (fig. 55). La surface A est d'abord exploitée et produit des éclats dont certains sont typologiquement Levallois, avant la rotation du bloc et l'exploitation de la surface B. Ce schéma opératoire a aussi été identifié par l'examen diacritique du remontage 7 (fig. 56). Le tailleur a installé sur le rognon un plan de frappe et exploité la première surface par une ou plusieurs séquences de base, dont seule la dernière est visible sur le nucléus. Ensuite, il a changé d'orientation, la surface inférieure devenant productive. L'axe du débitage bascule de $90^{\circ}$ et le nouveau plan de frappe est installé sur la périphérie de l'ancienne surface de production. Cette surface est traitée par au moins deux séquences de base produisant deux pointes typologiquement Levallois dont l'une remonte sur le nucléus.

Dans le second cas, représenté par 8 nucléus, les deux surfaces sont perpendiculaires ou sécantes. Dans ce dernier cas, elles se croisent selon un angle fermé (fig. 57). Les directions des enlevèments divergent. Chacune des surfaces sert successivement de plan de frappe et de surface de production. Une ou plusieurs séquences de base sont réalisées sur une première surface productive. Ensuite, une seconde surface est exploitée de la même manière. Le débitage s'effectuant en série, il peut à nouveau concerner la première surface exploitée, avant un retour à la seconde.

Le remontage 5 illustre la modalité unipolaire convergente avec deux surfaces productives débitées en alternance (fig. 58 et 59). Le choix du tailleur a porté sur un bloc de volume quadrangulaire présentant des arêtes naturelles (plans de clivage). La forme des blocs est liée à l'origine de la matière première qui se présente sous forme de plaquettes constituant des bancs dans la masse de calcaire de Saint-Ouen. 

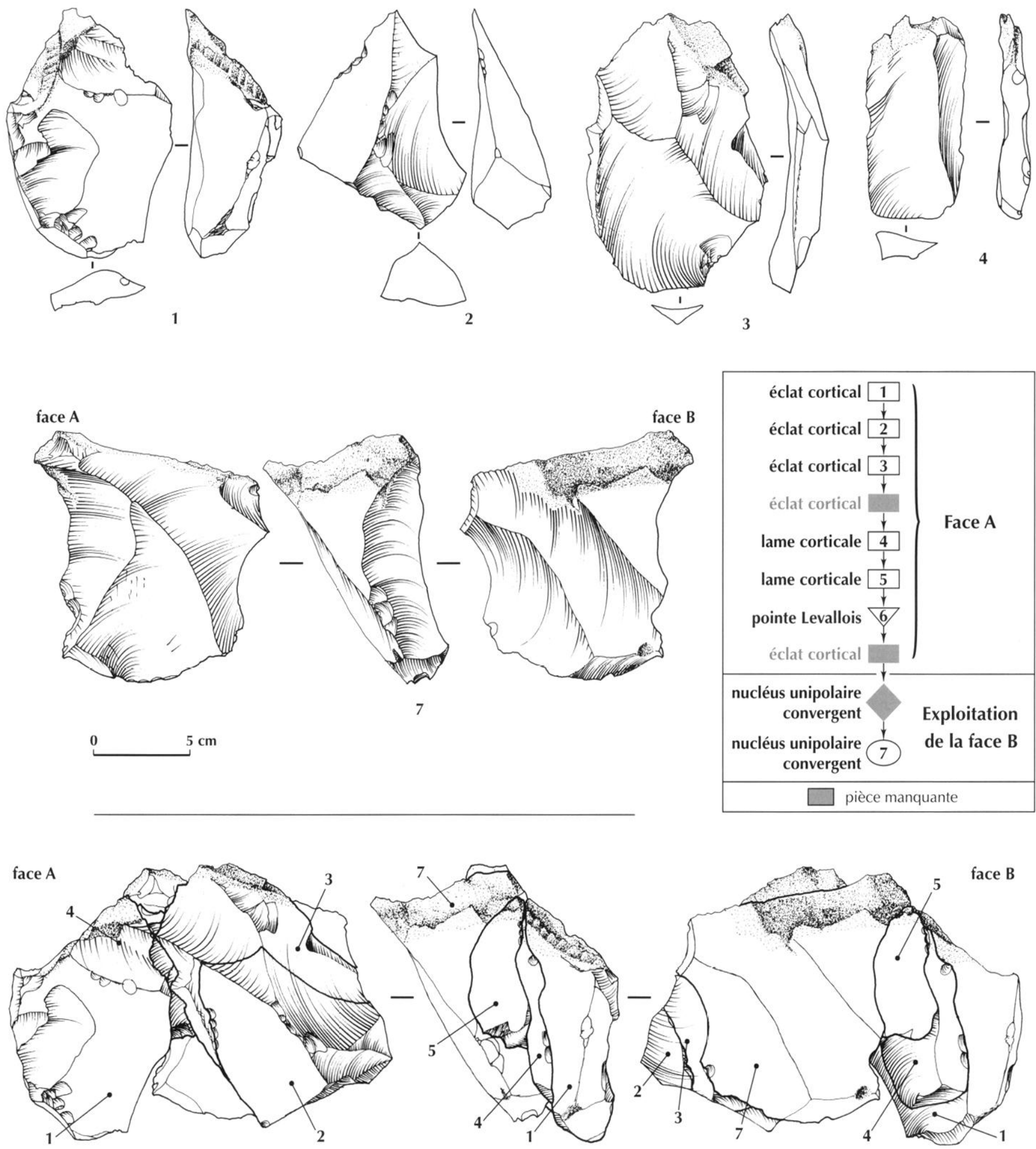

Fig. 55 - Remontage de l'ensemble 8. Les muméros indiqués sous les arlefacts lithiques renvoient à leur position dans le schéma diacritique et à la légende associée (dessin P. Raymond). 


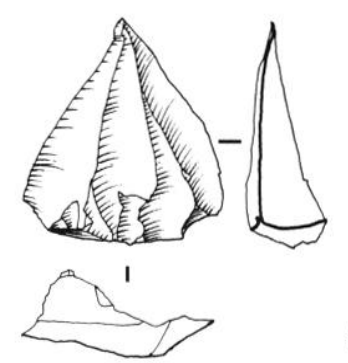

1
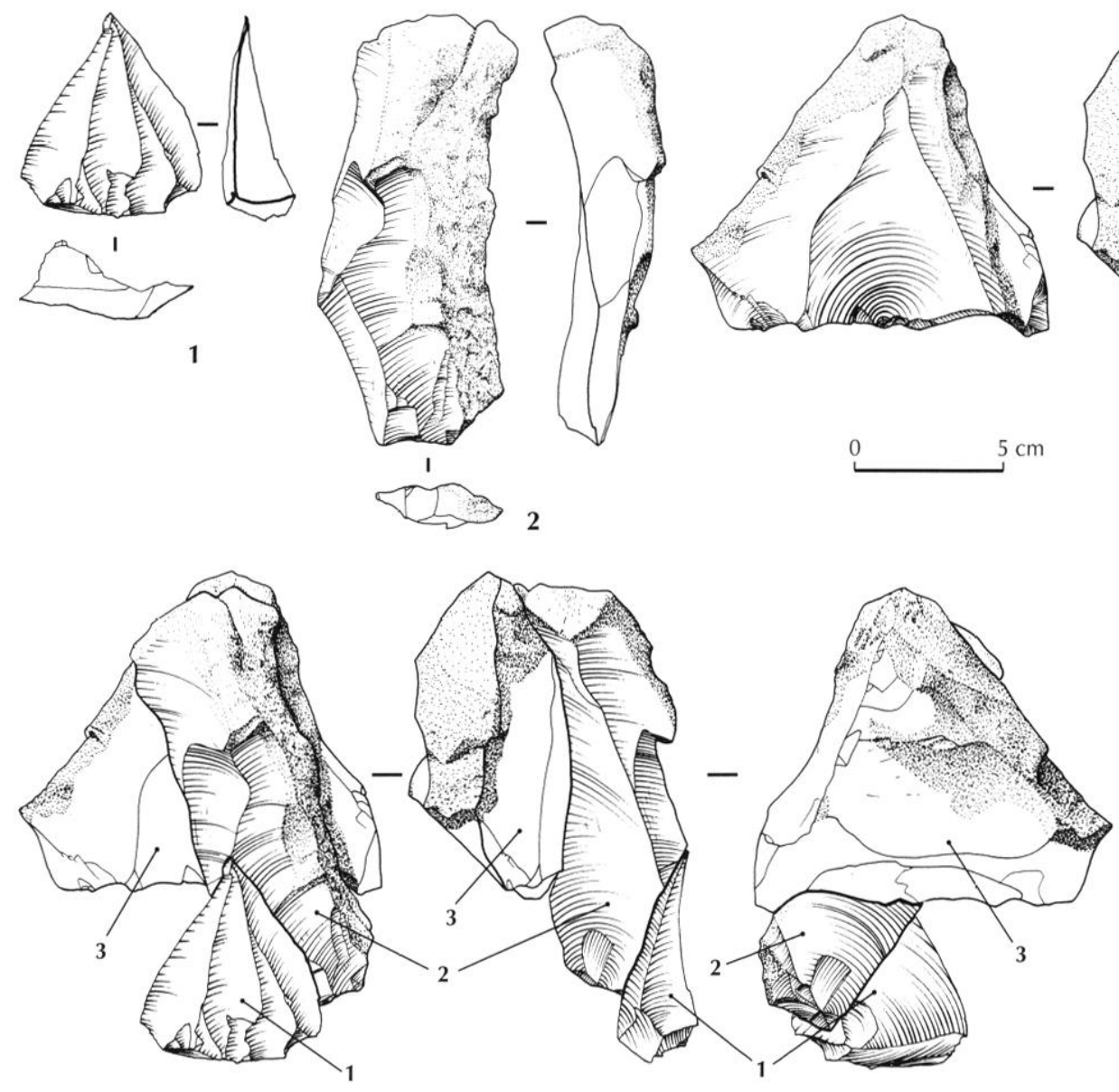

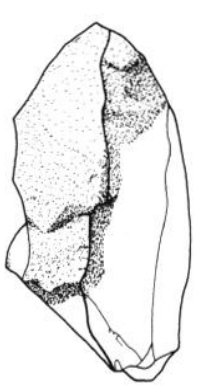

3
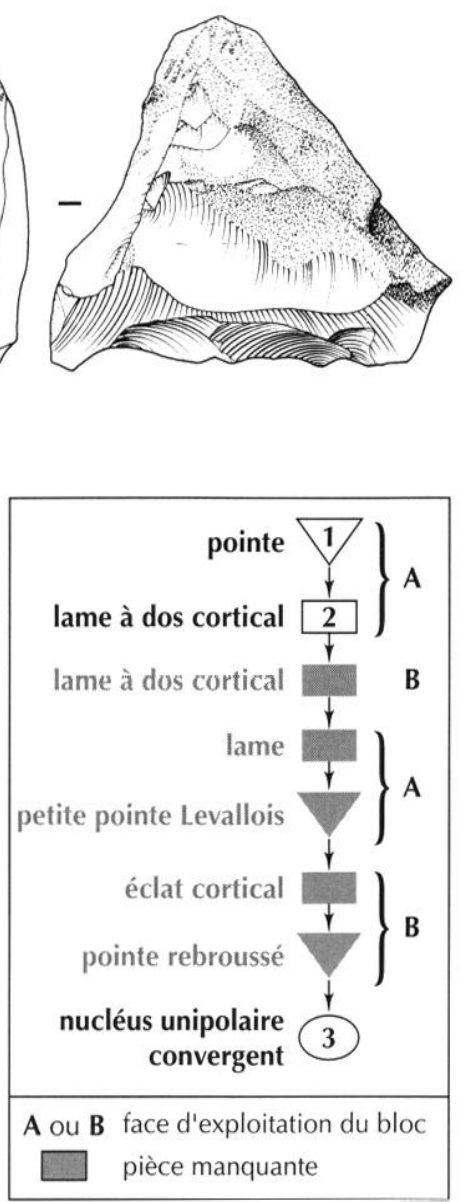

Fig. 56 - Remontage de l'ensemble 7. Les numéros indiqués sous les artefacts lithiques renvoient à leur position dans le schéma diacritique et à la légende associée (dessin P. Raymond).

La préparation de ce nucléus peut être décomposée en plusieurs phases :

1. Le débitage commence par l'enlèvement de la première série d'éclats corticaux débordants sur la surface A (fig. 58 et 59). Le tailleur utilise comme nervure guide l'arête naturelle du bloc. Le premier éclat cortical (fig. 58, $\mathrm{n}^{\circ}$ la) sert de nucléus. Après une préparation sommaire du plan de frappe, le tailleur obtient 3 enlèvements dont 2 ont pu être remontés. Il s'agit de deux enlèvements débordants (fig. 58, $\mathrm{n}^{\mathrm{os}} 1 \mathrm{~b}$ et $\left.1 \mathrm{c}\right)$. Le troisième produit, qui devait être une pointe pseudo-Levallois, n'a pas été retrouvé. Deux éclats corticaux sont ensuite détachés (fig. $58, \mathrm{n}^{\text {os }} 2$ et 3 ).

2. Le tailleur change de surface de production et enlève un éclat cortical sur la surface B (fig. $58, \mathrm{n}^{\circ} 4$ ). La surface A sert de plan de frappe sans aucune préparation préalable.
3. Le débitage recommence sur la surface A, la surface $B$ devenant la surface de préparation du plan de frappe. Cette séquence est composée de deux enlèvements dont le premier est un éclat cortical réfléchi (fig. $58, \mathrm{n}^{\circ}$ ) et le deuxième un éclat débordant (fig. 58 , $\left.\mathrm{n}^{\circ} 6\right)$.

4. Un grand éclat cortical débordant est détaché de la surface B (fig. $58, \mathrm{n}^{\circ} 7$ ), suivi d'un éclat cortical (fig. 58 , $\mathrm{n}^{\circ} 8$ ).

5. Sur la surface A, le tailleur a effectué 3 enlèvements : un éclat cortical (fig. $60, \mathrm{n}^{\circ} 9$ ) et deux éclats débordant (fig. $58, \mathrm{n}^{\text {os }} 10$ et 11 ).

Une fois le nucléus mis en forme, débute la phase de production qui a pour objectif l'obtention des pointes. Quatre séquences ont été distinguées avant l'abandon du nucléus.

La première séquence de production se déroule sur la surface B. Après avoir enlevé deux éclats laminaires 

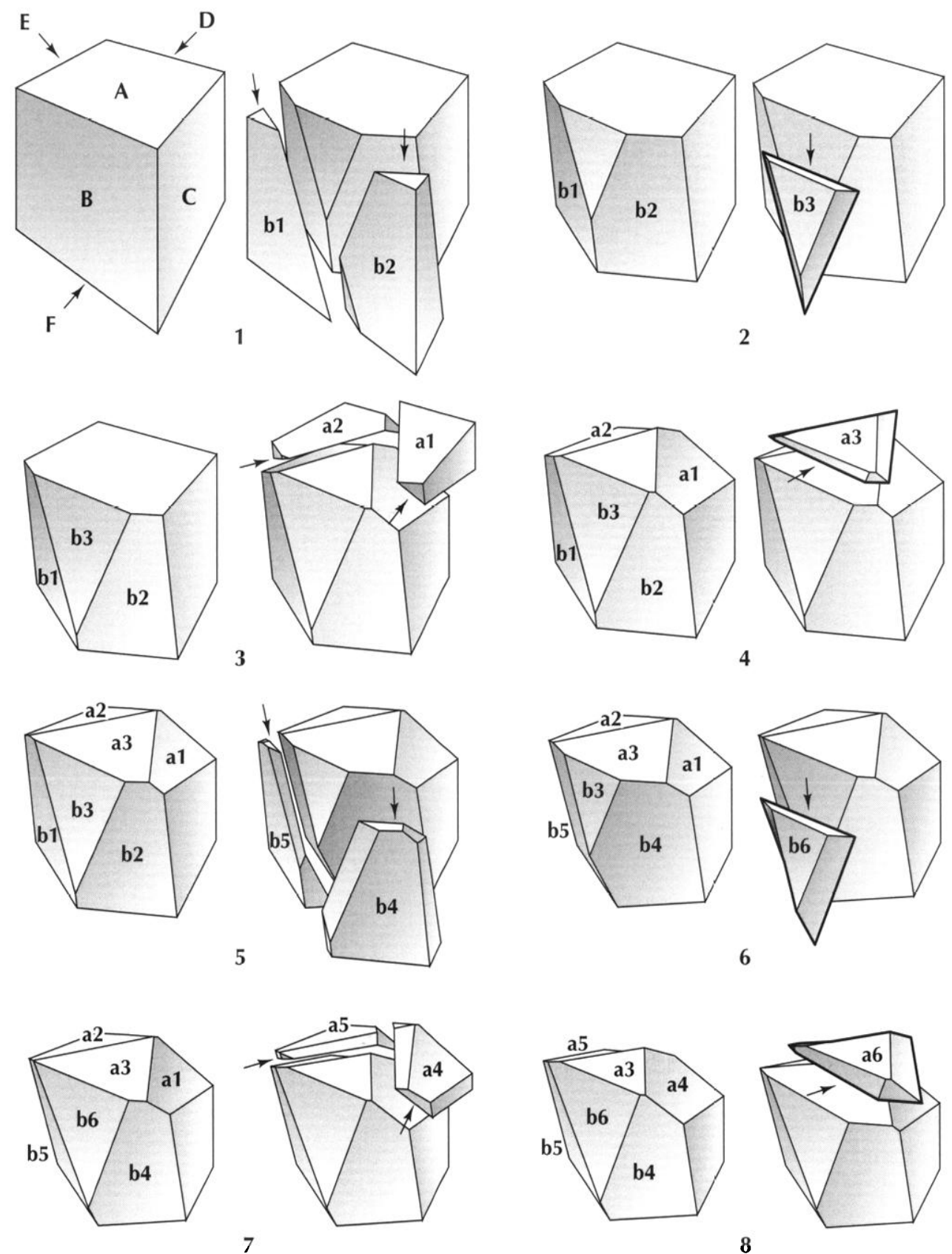

Fig. 57 - Débitage unipolaire convergent : schéma d'un bloc à deux surfaces perpendiculaires ou sécantes exploitées de façon alternative (dessin P. Raymond).

débordants (fig. $58, \mathrm{n}^{\text {os }} 10$ et 11 ) sur le même bord du nucléus et un troisième sur le bord opposé, le tailleur a obtenu deux pointes dont les négatifs sont visibles sur le bloc. La deuxième pointe devait être allongée et bien équilibrée au vu de son négatif. Une tentative de détachement d'une pointe sans la repréparation suffisante (enlèvements courts et réfléchis) des convexités a échoué. Le tailleur a obtenu un large éclat réfléchi, typologiquement Levallois (fig. $58, \mathrm{n}^{\circ} 14$ ). Il présente un talon sommairement préparé (larges facettes). Le coup a été porté sur la droite du point d'impact de la pointe.

Après l'accident, le tailleur a changé de surface et est revenu sur la surface $\mathrm{A}$. Il y a effectué une séquence de base composée de deux enlèvements débordants et d'une pointe. Seul le premier éclat débordant (fig. 58, 

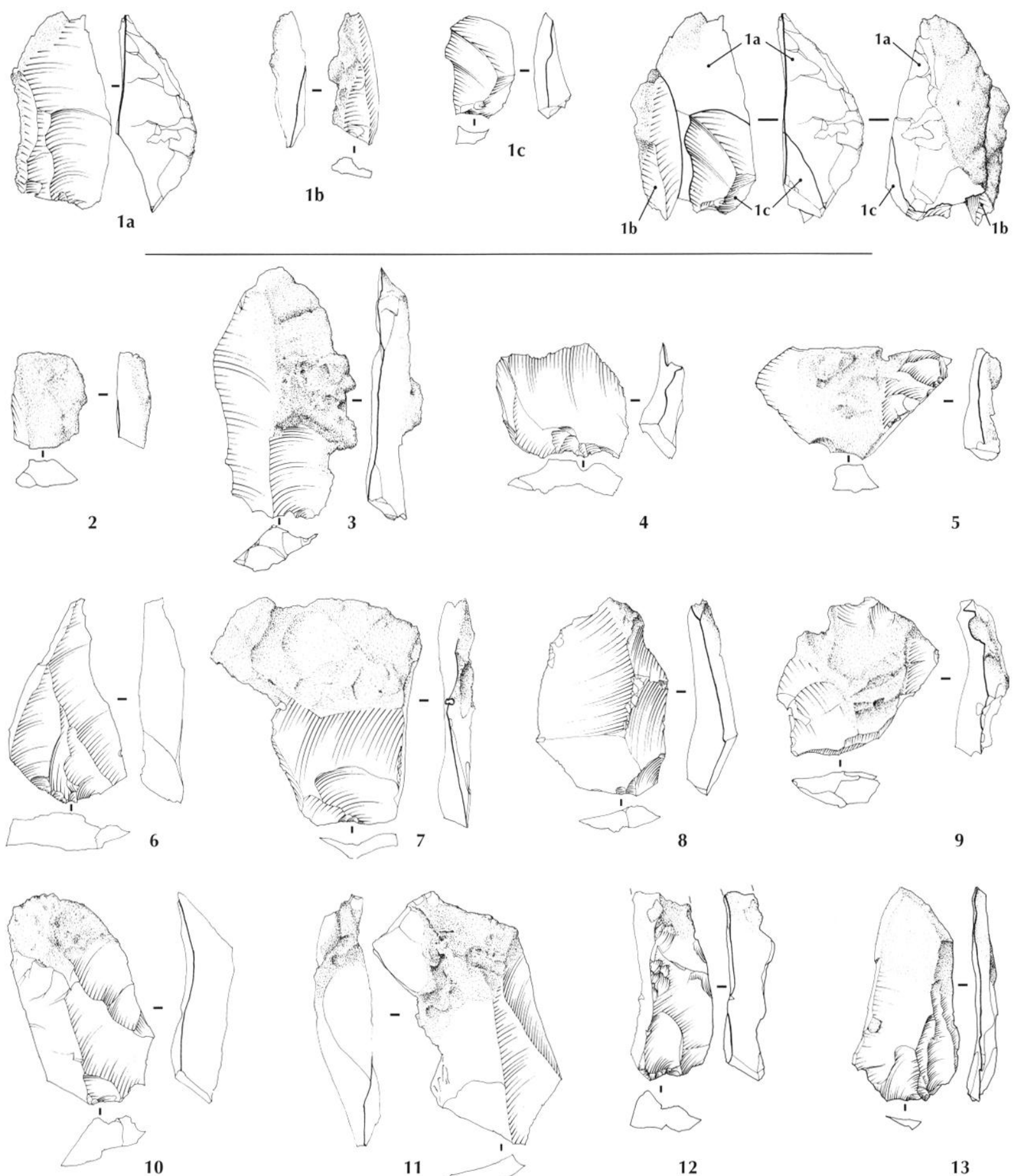

11

12
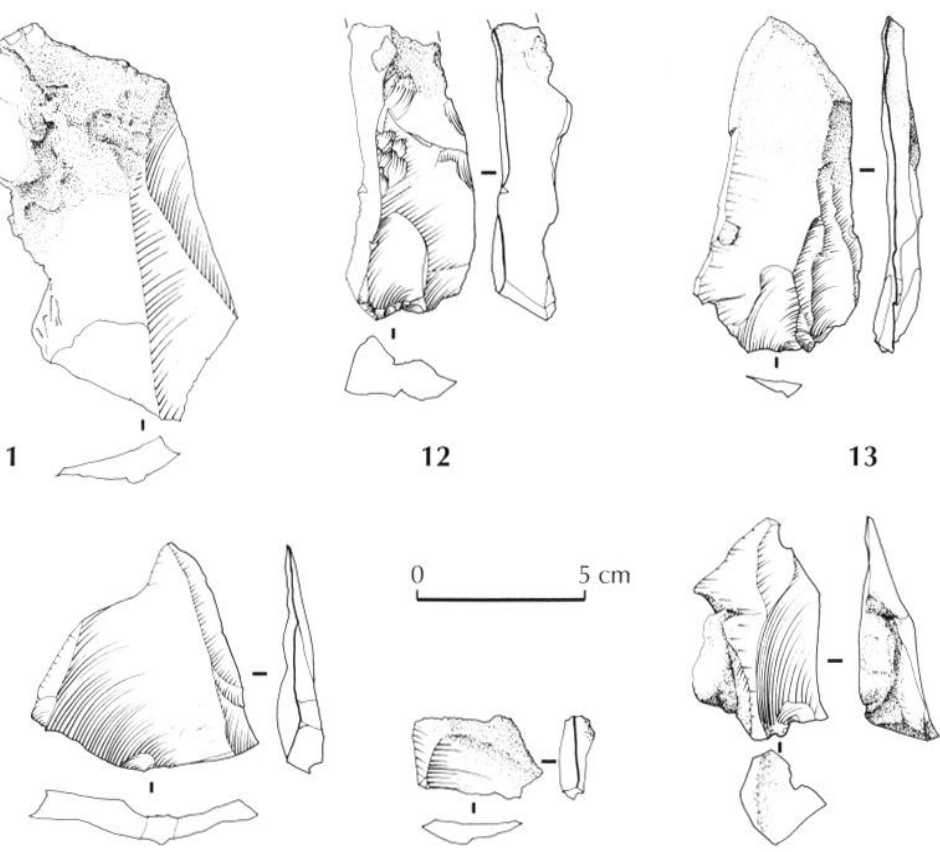

13

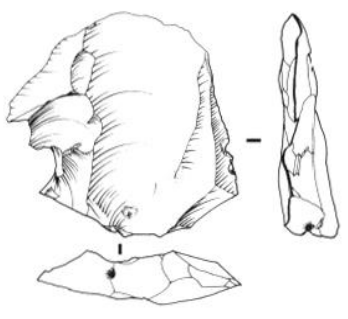

14

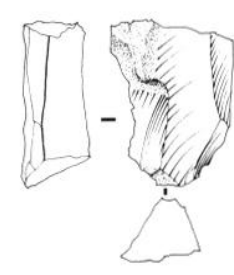

15

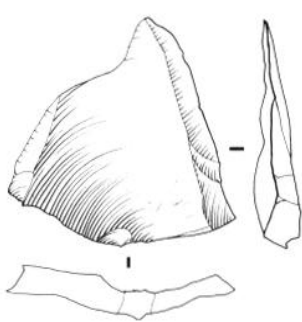

16

17

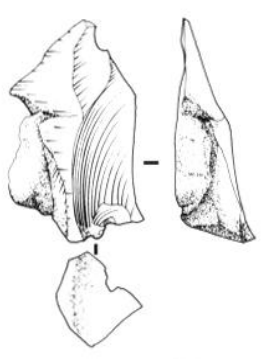

18

Fig. 58 - Remontage de l'ensemble 5 : Les numéros indiqués sous les artefacts lithiques renvoient à leur position dans le schéma diacritique (fig. 59) et à la légende associée (dessin P. Raymond). 

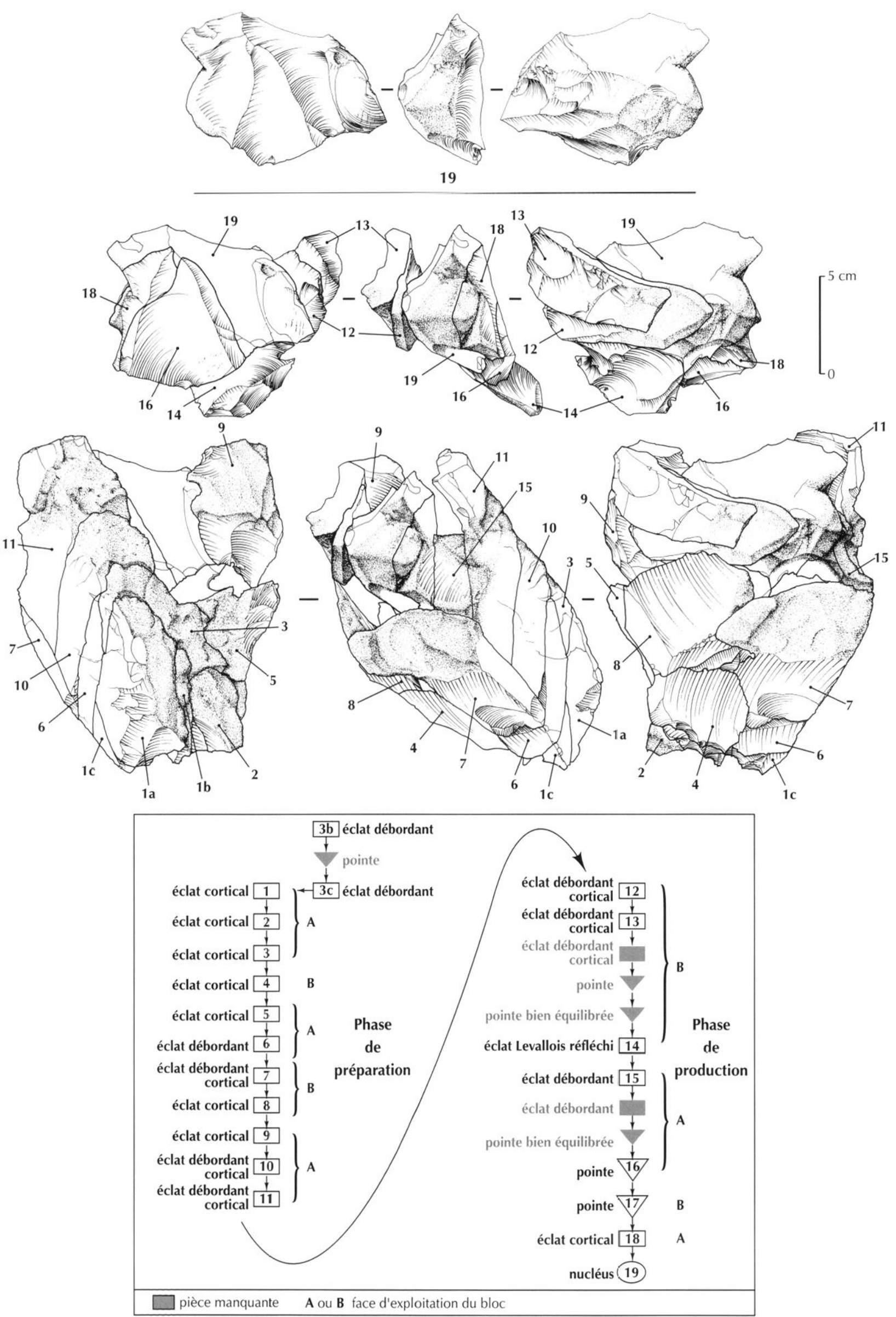

Fig. 59 - Remontage de l'ensemble 5 (dessin P. Raymond). 
$\mathrm{n}^{\circ}$ 15) a été remonté sur le nucléus. Comme dans la séquence précédente, le tailleur a tenté le détachement d'une deuxième pointe sans la repréparation des convexités. Le point d'impact est légèrement décalé sur la droite comme sur la surface B. La pointe obtenue (fig. $58, \mathrm{n}^{\circ} 16$ ) est légèrement réfléchie. Elle présente un talon facetté, mais il s'agit des négatifs de la précédente séquence productive effectuée sur la surface A. Cette pointe est le seul produit du débitage retrouvé en dehors de l'amas 1 , à près de $15 \mathrm{~m}$ de celui-ci. Elle ne présente ni retouche ni trace d'utilisation.

Après l'obtention de la pointe, le tailleur a changé de surface et détaché le dernier éclat cortical sur la surface $B$ (fig. $58, \mathrm{n}^{\circ} 17$ ), puis est retourné à la surface $A$ et a obtenu un éclat à dos cortical sur le même côté, qui a laissé un contre-bulbe bien prononcé (fig. $58, \mathrm{n}^{\circ} 18$ ). Le nucléus fut alors abandonné après cette troisième séquence productive.

Le bloc remonté se compose ainsi de 19 sous-produits de débitage (éclats débordants et corticaux) et d'un produit prédéterminé (pointe). Après l'analyse du bloc (présence des négatifs), on peut estimer sa production à quatre supports de type pointe Levallois dont une seule a pu être remontée (fig. 59).

L'ensemble 10, provenant de l'amas 2, est à l'origine un rognon oblong de silex tertiaire, de grandes dimensions ( $\mathrm{L}: 30 \mathrm{~cm}, 1: 16 \mathrm{~cm}$ ) (fig. 60 et 61 ). La matière première est de mauvaise qualité en raison de la présence de plusieurs impuretés à l'intérieur du bloc. Le cortex est assez frais et épais. La présence d'une grande géode au milieu du rognon a provoqué une cassure au tout début de l'épannelage. À partir de ce moment, les deux volumes I et IV ont été débités séparément, mais selon un schéma opératoire similaire. Deux autres fragments (II et III), de petites dimensions, ont été exploités de façon sommaire par le biais d'un débitage unipolaire convergent.

Le bloc I a été décortiqué et un plan de frappe mis en place. Il est installé sur une ancienne surface de clivage. De là partent les premiers enlèvements corticaux qui mettent en place des convexités latérales sur la première surface de production A.

Une première séquence a produit une série de pointes et éclats Levallois ou pseudo-Levallois sur la face A (fig. 61). Puis a suivi une séquence de production sur la surface B, la surface A servant de plan de frappe pour la surface $B$. Le tailleur a obtenu des produits à dos cortical et une pointe grossière.
Ensuite deux éclats ont été détachés sur la surface A, puis le nucléus a été abandonné. Les produits obtenus sont, dans la même séquence, des pointes typologiquement Levallois et des pointes pseudo-Levallois quand le coup de percuteur est porté dans une direction cordale (Boëda, 1993). Les talons sont en majorité lisses.

Le second bloc (IV) a tout d'abord subi une réduction importante par de grands enlèvements unipolaires. Le débitage est difficile à contrôler en raison des impuretés à l'intérieur du bloc qui rendent les enlèvements aléatoires. Des éclats Levallois et des pointes pseudo-Levallois ont été produits en alternance sur les deux surfaces inscrites dans les plans croisés.

Cet ensemble démontre que des pointes pseudoLevallois peuvent être produites par ce schéma opératoire. Le coup de percuteur, porté cordalement par rapport aux nervures guides, détache une pointe dont l'axe morphologique est décalé par rapport à l'axe de débitage. Le coup de percuteur est porté, dans ce cas précis, parallèlement au plan de la surface exploitée.

Les remontages précédemment décrits ont démontré que plusieurs surfaces de production pouvaient s'inscrire dans le volume du bloc, au cours de son processus de réduction, sans qu'il y ait rupture dans le système productif. Ces observations tendent à s'éloigner du concept du débitage Levallois tel qu'il est classiquement défini (Boëda, 1994).

\section{La chaîne opératoire à éclats}

Cette chaîne opératoire n'est représentée que par 48 nucléus qui présentent un débitage organisé. Elle est minoritaire par rapport à la production des pointes Levallois. Quatre schémas de production ont été identifiés.

\section{Schéma unipolaire parallele (25 nucléus)}

À partir d'un plan de frappe sommairement installé, quelques enlèvements de direction parallèle sont détachés. L'exploitation du bloc s'arrête rapidement, en raison de la morphologie ou de la qualité du matériau. Dans six cas, le débitage est réalisé aux dépens de la face ventrale d'un éclat.

Le remontage de l'ensemble 6 a permis de reconstituer quasi entièrement un bloc de morphologie régulière (fig. 62). Le débitage a été mené à partir d'un seul plan de frappe, en profitant des arêtes de ce 


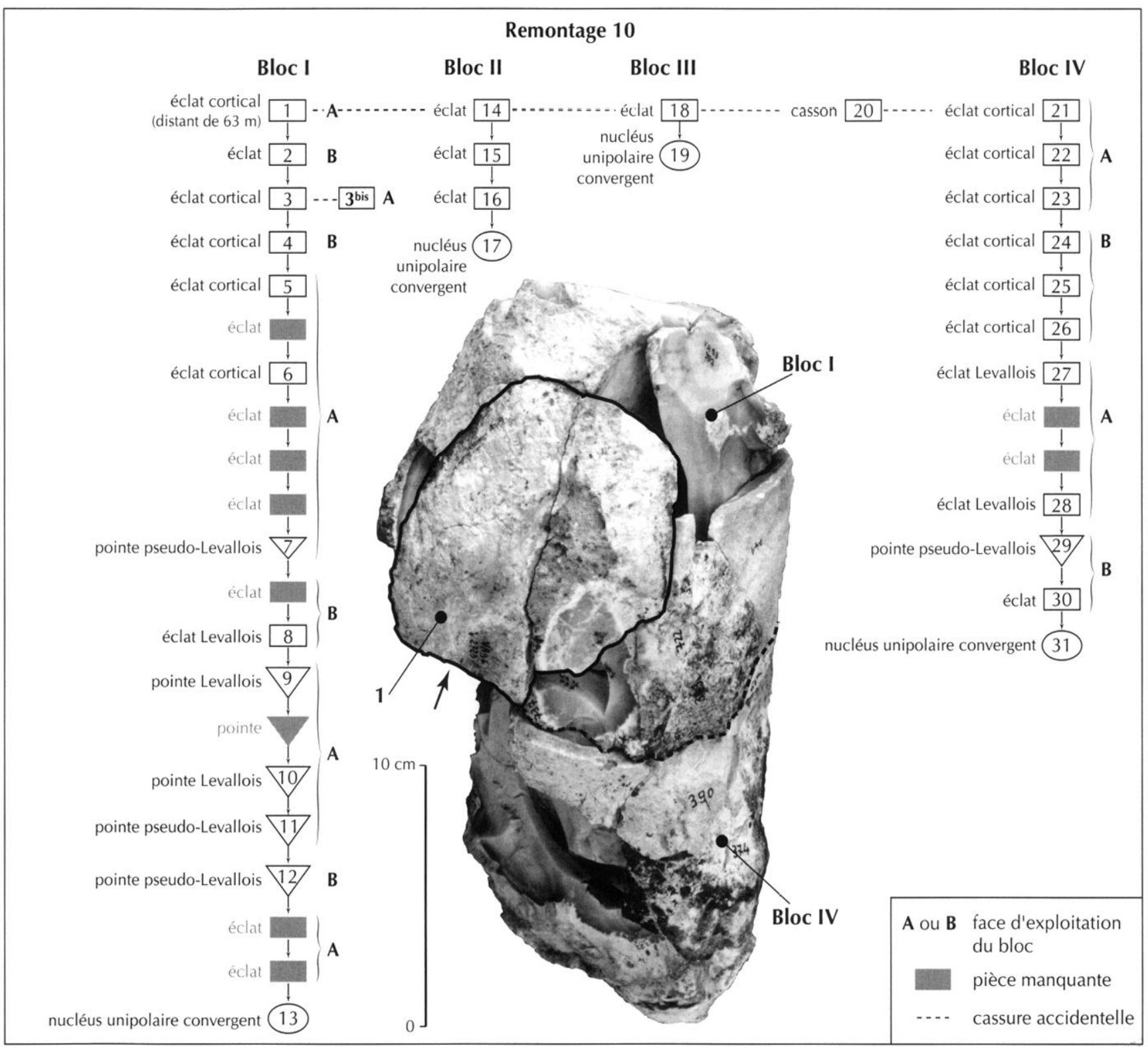

Fig. 60 - Remontage de l'ensemble 10 (dessin P. Raymond).

rognon quadrangulaire. Des enlèvements à arêtes plus ou moins parallèles ont été détachés, outrepassant légèrement, mais de façon systématique, en raison des dimensions et de l'orientation de la surface productive.

\section{Schéma Levallois récurrent centripète (11 nucléus)}

Les nucléus, de dimensions et de morphologie similaires, sont constitués de deux surfaces opposées et hiérarchisées (Boëda, 1994). La première sert de plan de frappe périphérique pour l'exploitation de la seconde.
Les traces de préparation de convexités ont le plus souvent été ôtées par l'enlèvement des éclats Levallois. L'axe de percussion est parallèle au plan sécant qui sépare les deux surfaces (fig. $63, \mathrm{n}^{\text {os }} 1$ à 3 ). Les remontages font défaut sur les nucléus de ce type. L'un d'entre eux a été réalisé sur la face ventrale légèrement bombée d'un grand éclat (fig. 63, $\mathrm{n}^{\circ} 1$ ). Neuf de ces nucléus sont en silex tertiaire, le dixième en silex secondaire. Ce dernier, de modalité récurrente centripète, a été exploité jusqu'au moment où de profonds contre-bulbes ont empêché la poursuite du débitage. 


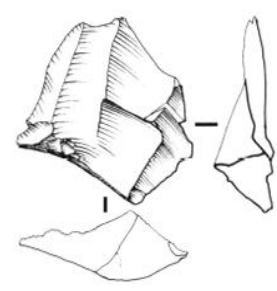

7

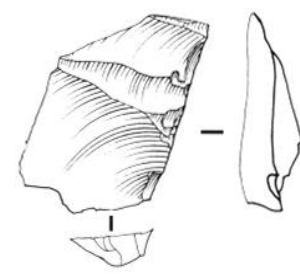

11
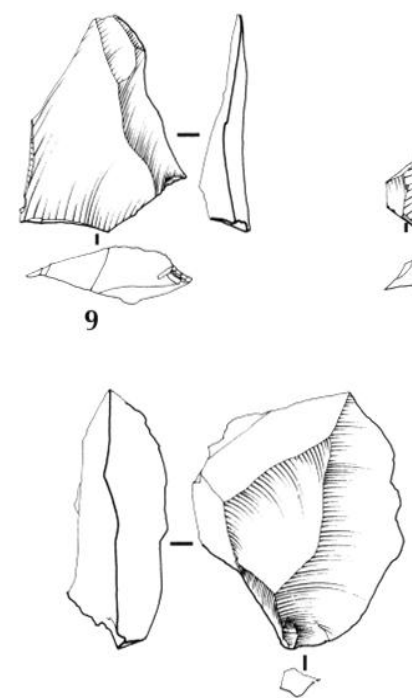

12

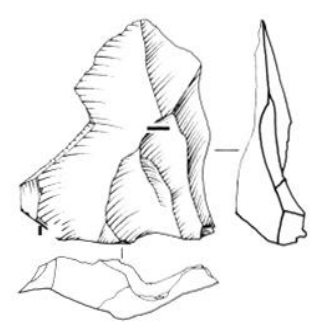

10

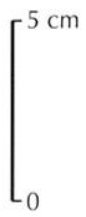

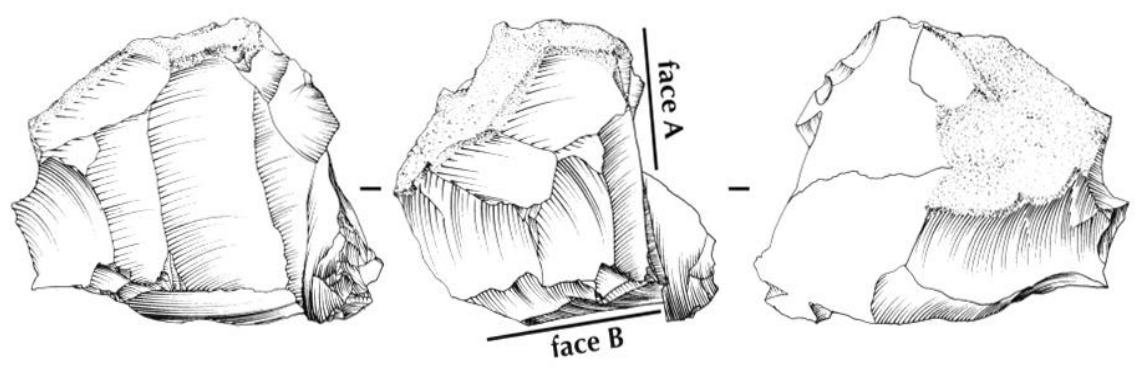

13
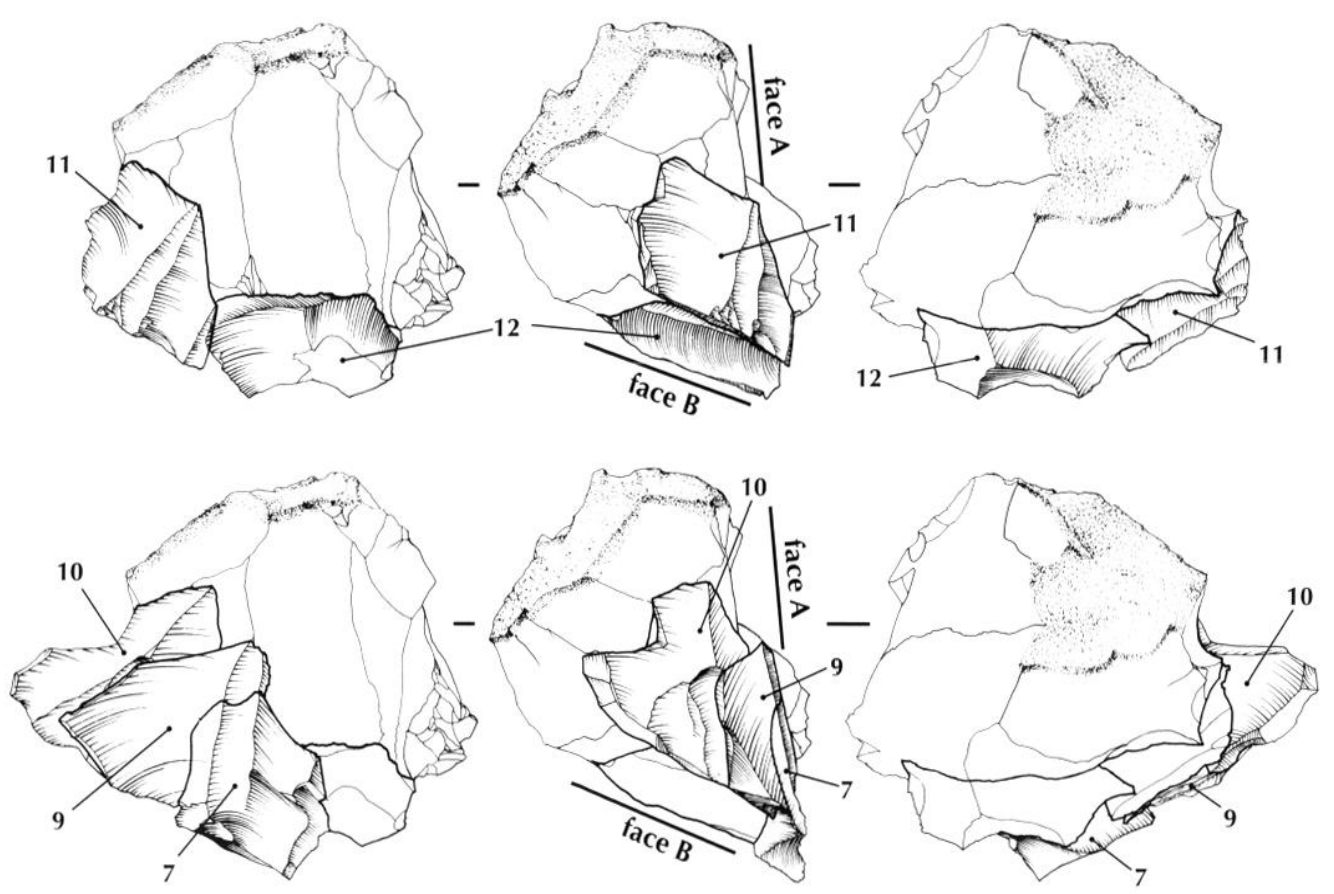

Fig. 61 - Remontage de l'ensemble 10. Les numéros indiqués sous les artefacts lithiques renvoient à leur position dans le schéma diacritique (fig. 60) et à la légende associée (dessin P. Raymond). 


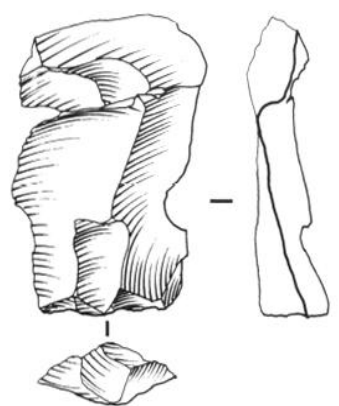

6
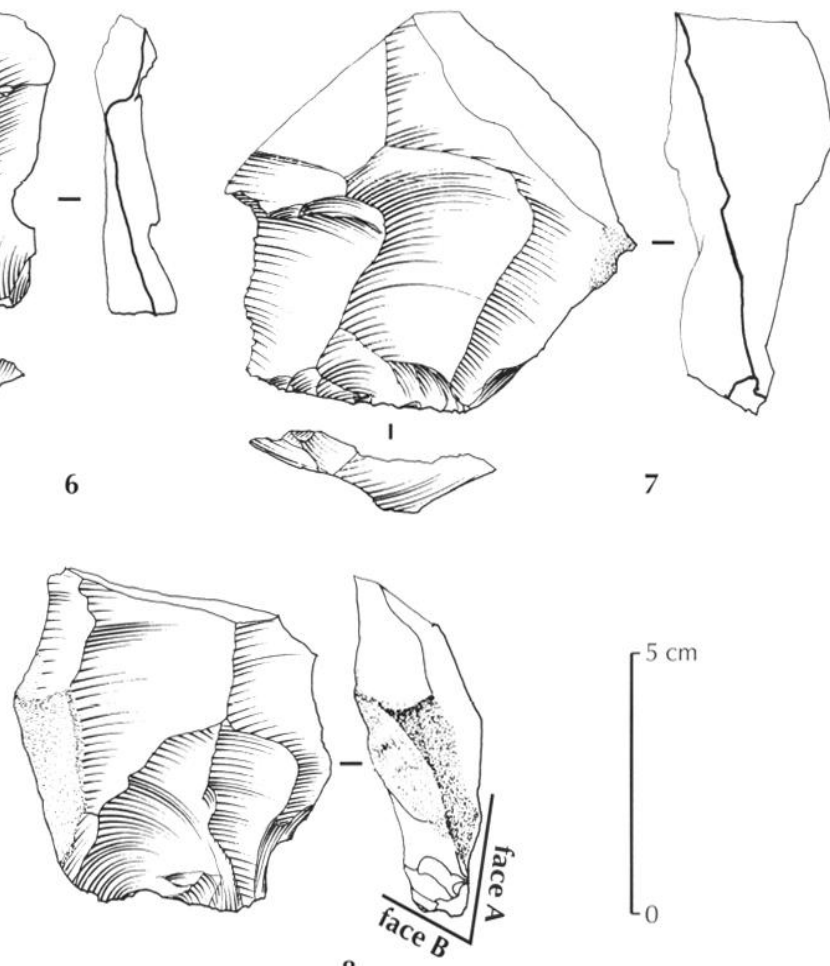

8
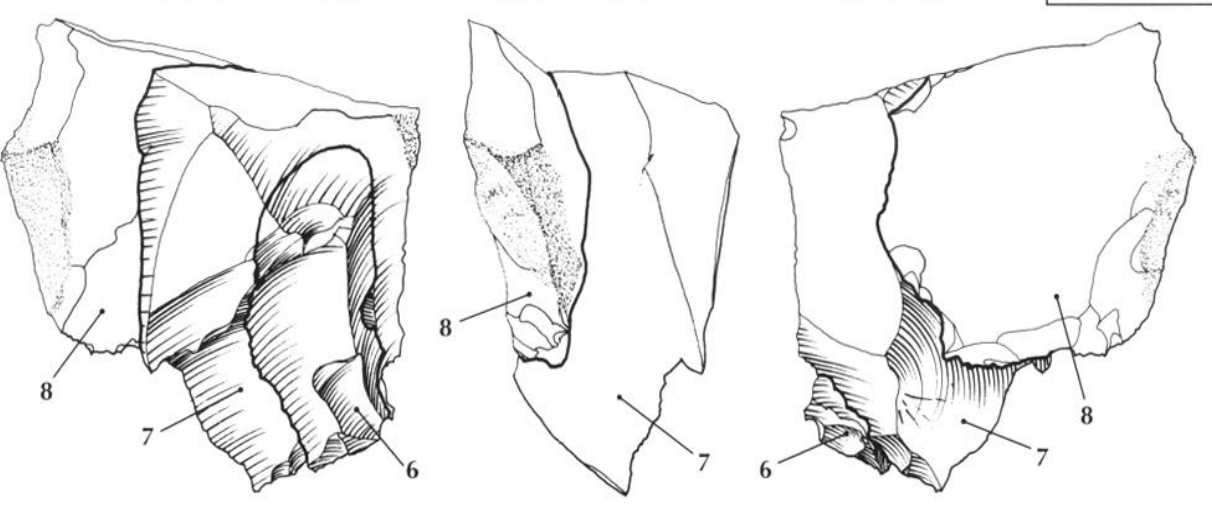

pièce manquante

A ou $\mathbf{B}$ face d'exploitation du bloc nucléus 8
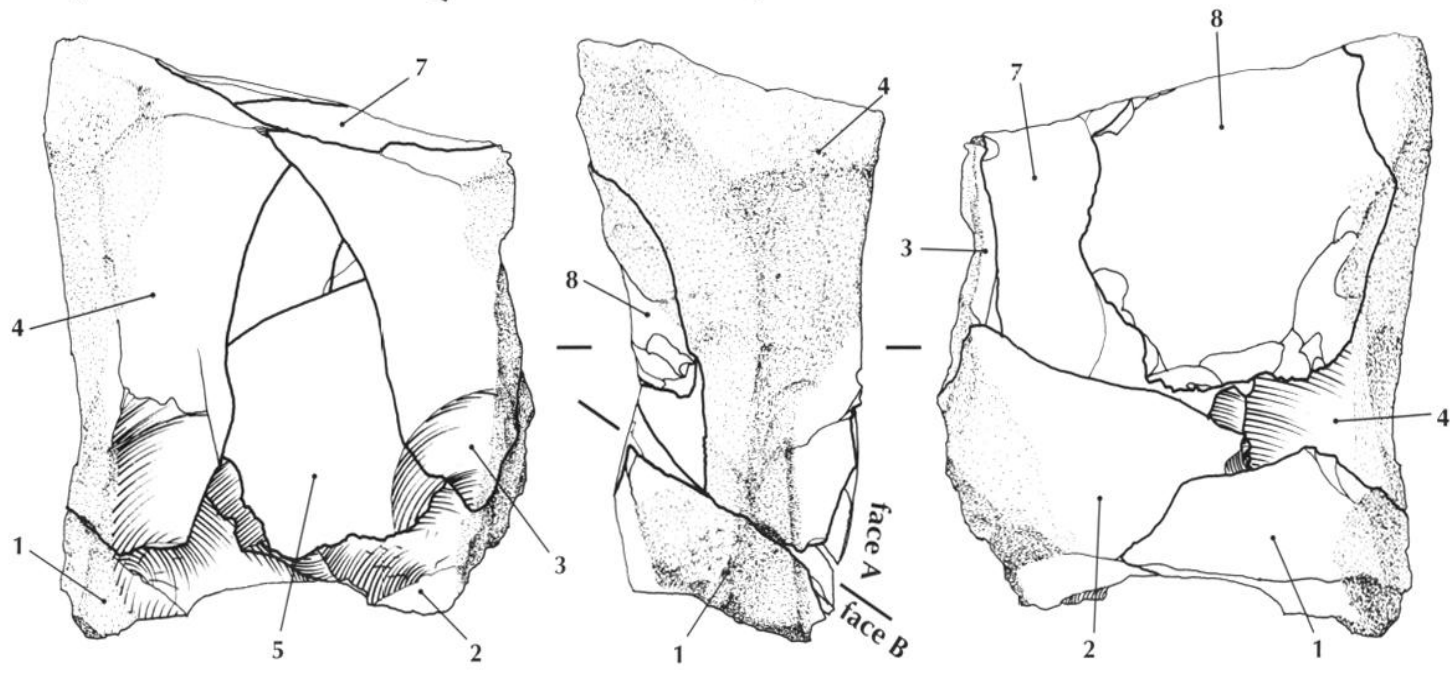

Fig. 62 - Remontage de l'ensemble 6. Les numéros indiqués sous les artefacts lithiques renvoient à leur position dans le schéma diacritique et à la légende associée (dessin P. Raymond). 

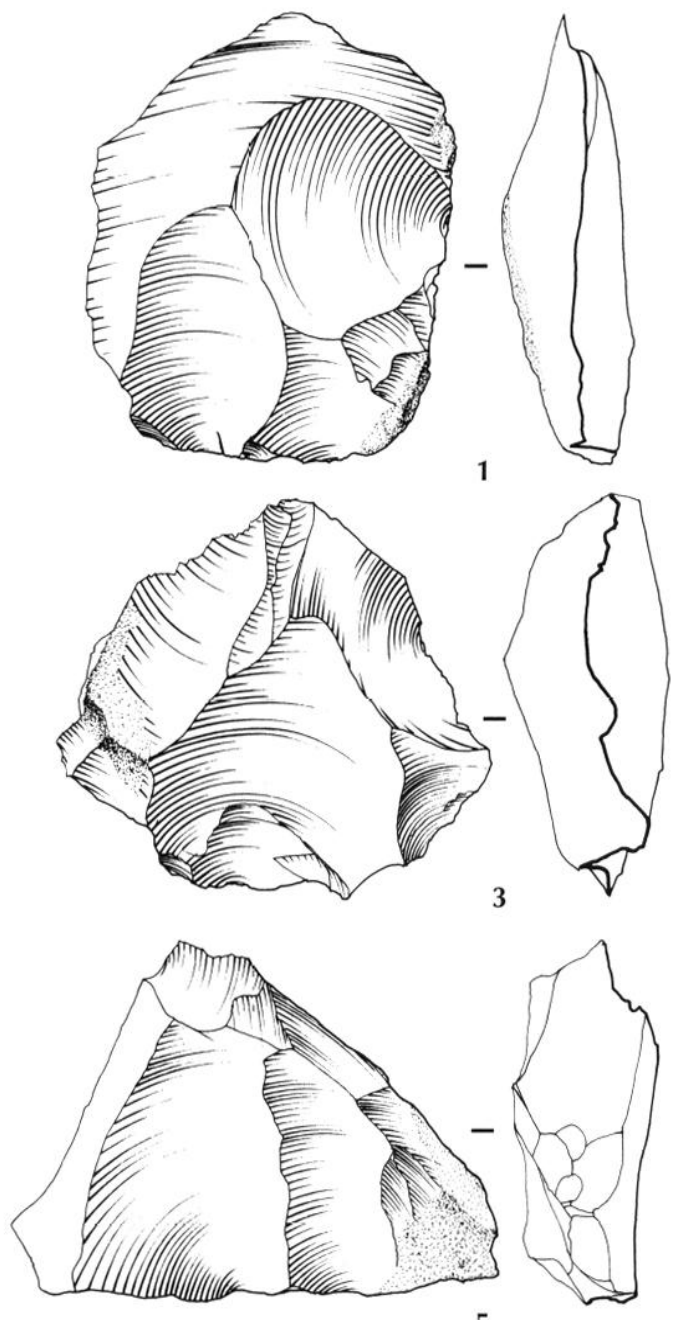

5
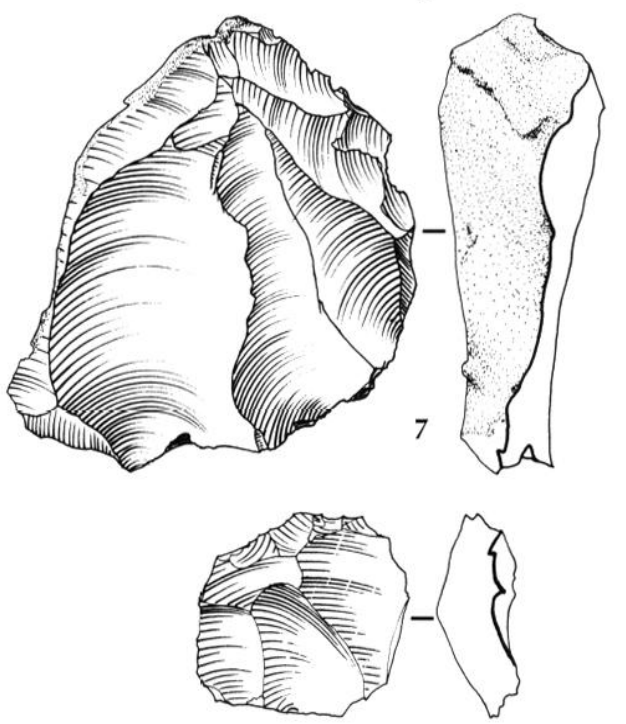

9

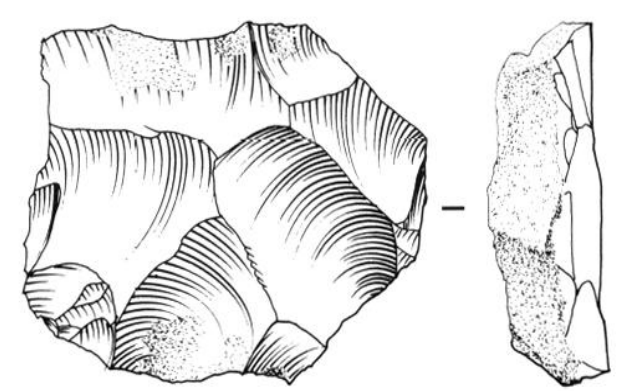

2
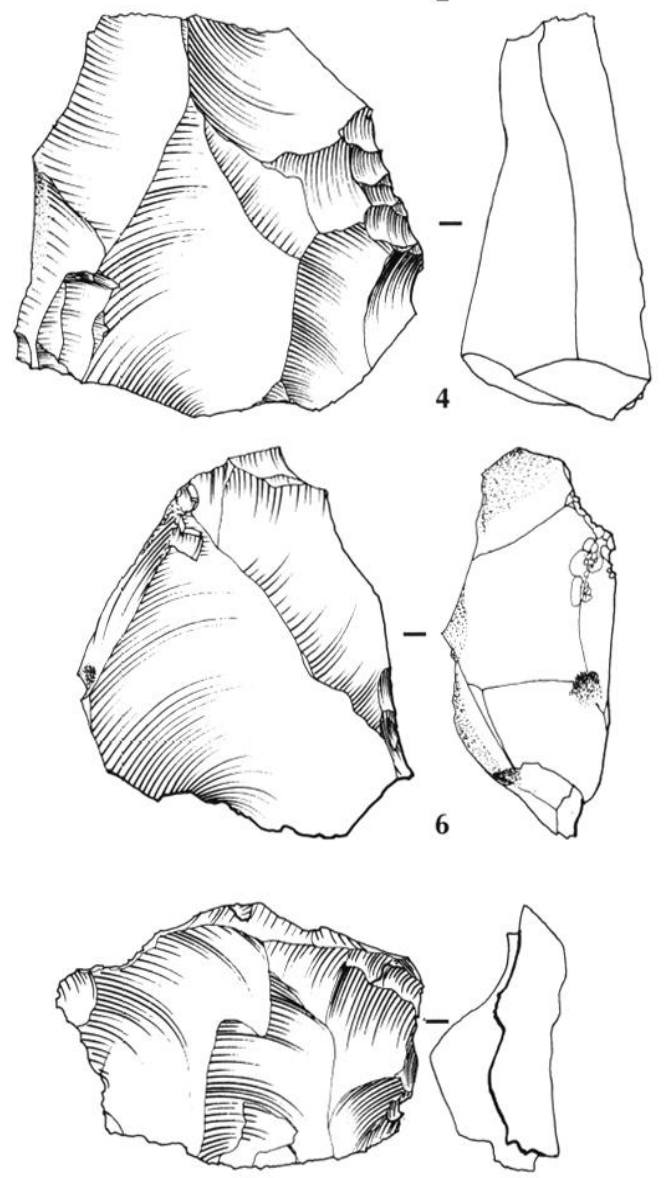

8

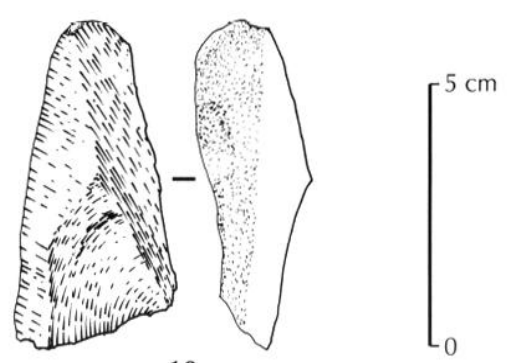

10

Fig. 63 - Nucléus : 1, nucléus Levallois récurrent centripète sur éclat ; 2, 3, nucléus Levallois récurrent centripète; 4, nucléus Levallois récurrent bipolaire; 5, 6, nucléus à pointes, avec enlèvements provenant d'un plan de frappe opposé, pour ôter une arête gênante; 7, nucléus unipolaire convergent en silex secondaire; 8 , nucléus Levallois récurrent centripète sur éclat; 9, nucléus bipolaire; 10, nucléus unipolaire convergent en grès (dessin P. Raymond). 


\section{Schéma Levallois récurrent bipolaire (3 nucléus)}

L'exploitation s'effectue à partir de deux plans de frappe opposés. Un de ces nucléus, complètement désilicifié, est d'une lecture diacritique très difficile. Sur la surface productive d'un autre de ces nucléus, l'exploitation s'est terminée avec le détachement d'un éclat pointu à la faveur de deux arêtes convergentes (fig. $63, n^{\circ} 4$ ). Le troisième nucléus porte également le négatif d'un dernier enlèvement envahissant.

\section{Nucléus bipolaires à éclats (9 nucléus)}

Les blocs d'origine sont de petite taille, en silex tertiaire (5) ou secondaire (1). Le débitage a débuté sans qu'aucune trace de préparation de convexités ne soit attestée. Les deux plans de frappe, opposés, ont été sommairement préparés, la percussion s'est également effectuée sur des surfaces naturelles corticales ou non. L'un de ces nucléus (remontage 23) (fig. 64) a produit, lors de la même séquence de débitage, des éclats dont certains exemplaires allongés peuvent s'apparenter à des lames, et des pointes.

\section{Nucléus informes (38 nucléus)}

Quelques enlèvements ont été faits sans aucune organisation sur des rognons de forme et de taille diverses, mais de médiocre qualité. Sur la face ventrale d'un éclat, un ou deux enlèvements ont parfois été débités à partir d'un plan de frappe préparé le plus souvent par un seul enlèvement.

\section{Les produits}

L'obtention de pointes, au nombre de 61 , constituait l'objectif prioritaire des tailleurs de Villiers-Adam. Elles ont surtout été produites par des méthodes unipolaires convergentes (fig. 65, $\mathrm{n}^{\text {os }} 1$ à 14). Elles peuvent être obtenues de manière fortuite dans une séquence de production laminaire, comme c'est le cas dans l'ensemble 23 décrit précédemment. Elles portent toutes les stigmates d'un débitage unipolaire. La grande majorité de ces pointes (52) sont en silex tertiaire, 8 sont en silex secondaire et une seule est en grès. Elles sont relativement peu nombreuses en regard de la totalité des artefacts $(2,78 \%)$, et surtout des nucléus unipolaires convergents dont elles sont issues (73). L'hypothèse d'un déplacement en dehors de l'aire de fouille, voire peut-être sur un autre site, peut être envisagée.
Les lames sont au nombre de 159. Le plus souvent à dos cortical, ce sont des lames au sens morphométrique du terme, mais elles proviennent pour la plupart de l'aménagement et de l'entretien des convexités des nucléus unipolaires convergents. 125 lames sont en silex tertiaire, 29 en secondaire et 6 en grès. Certaines lames portent des négatifs d'enlèvements opposés, qui témoignent de l'exploitation du bloc au moyen de deux plans de frappe (fig. $66, n^{\text {os }} 2$ et 4 ). Elles pourraient provenir d'un nucléus à débitage semi-tournant ou tournant. Elles témoigneraient de la connaissance de ce type de débitage, fréquent au Début glaciaire weichsélien dans le nord-ouest de la France, bien qu'aucun exemplaire de ce type n'ait été retrouvé dans ce secteur.

Les éclats Levallois $(n=84)$ sont de taille réduite. Ils sont en silex tertiaire (68), secondaire (15) ou en grès (1). Ils peuvent être les produits de l'exploitation des nucléus Levallois centripètes récurrents et portent dans ce cas des négatifs d'enlèvements convergents sur la face dorsale. Les exemplaires détachés à partir de nucléus en silex secondaire sont de belle facture. Quelques éclats Levallois ont été produits à partir de nucléus bipolaires (fig. 67).

Le débitage unipolaire convergent a aussi produit des éclats réguliers, typologiquement Levallois. Les négatifs d'enlèvements prédéterminants sont alors unipolaires et ont été détachés à partir du même plan de frappe que l'éclat Levallois (fig. $58, \mathrm{n}^{\circ} 14$ ), lors d'une même séquence de production que les lames et les pointes.

Les pointes pseudo-Levallois de cette série ont été produites par débitage unipolaire convergent, l'axe de percussion étant décalé par rapport à la surface productive (fig. $65, \mathrm{n}^{\text {os }} 15$ et 16). Elles peuvent avoir été produites lors de la même séquence que les pointes typologiquement Levallois. Elles sont en silex tertiaire (17) et secondaire (4). Les éclats débordants, au nombre de 24 , semblent provenir d'un débitage Levallois récurrent centripète, d'après leur morphologie et l'orintation des négatifs visibles sur leur face supérieure. Ils servent à aménager les convexités latérales de la surface de production. Aucun remontage n'est cependant venu étayer cette hypothèse. Ils sont tous en silex tertiaire.

\section{L'outillage}

L'outillage n'est constitué que de 62 pièces retouchées, ce qui ne constitue que $2,85 \%$ de la série (tabl. XIV). 


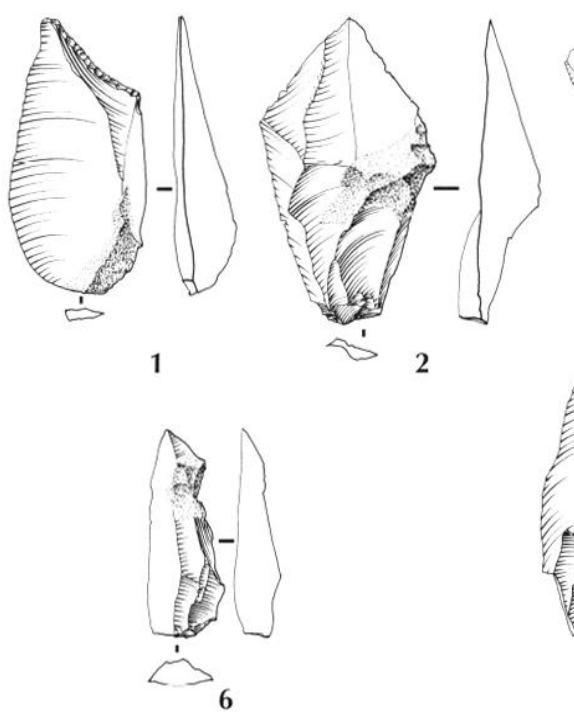

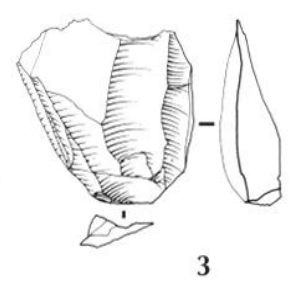

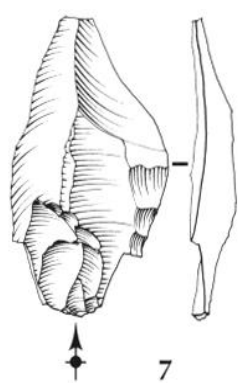

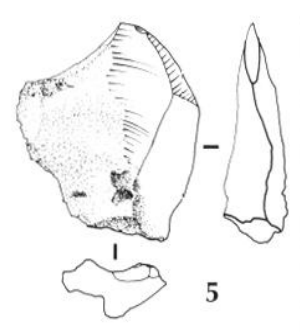

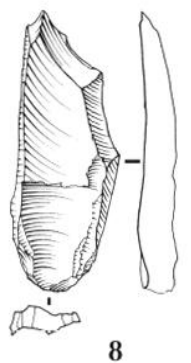

8

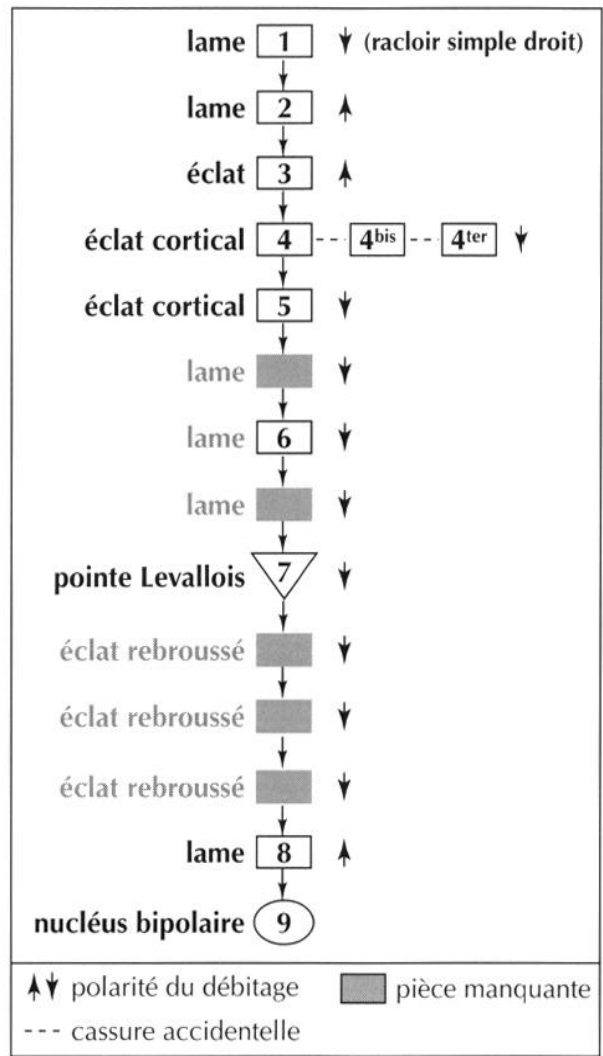

9
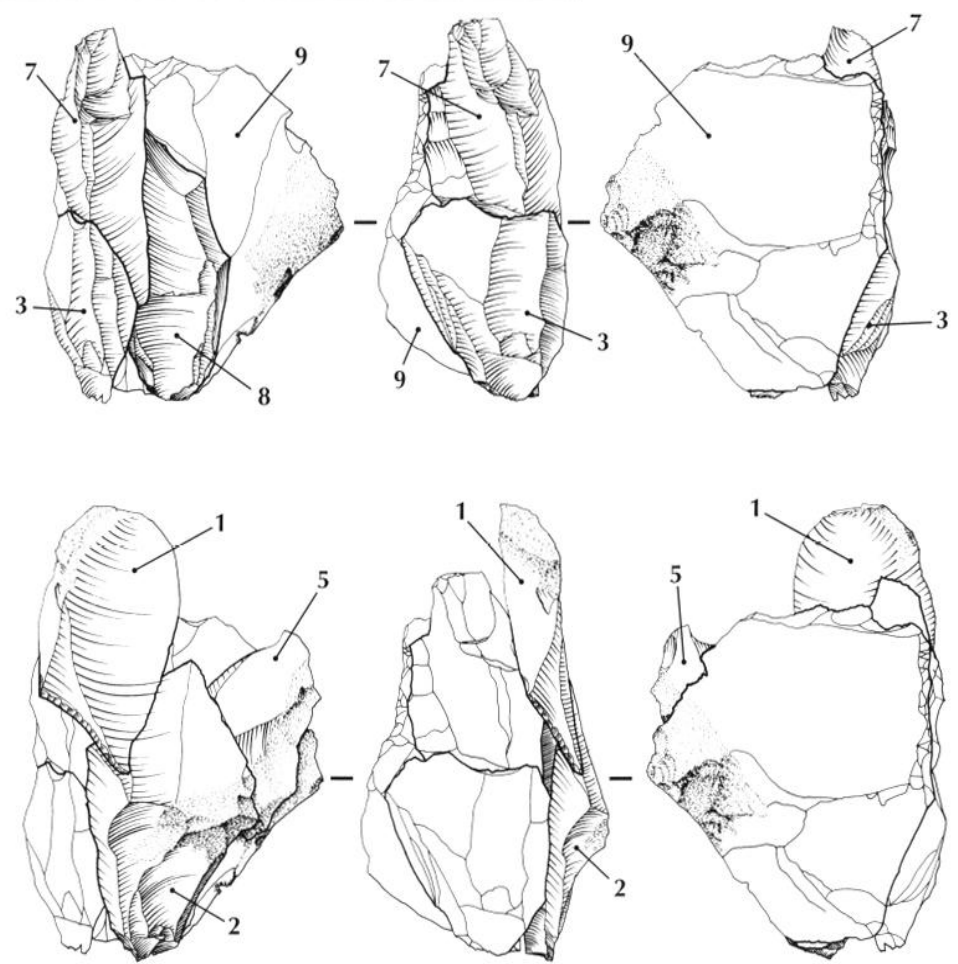

Fig. 64 - Remontage 23. Les numéros indiqués sous les artefacts lithiques renvoient à leur position dans le schéma diacritique et à la légende associée (dessin P. Raymond). 


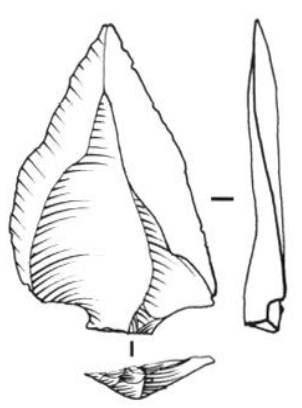

1
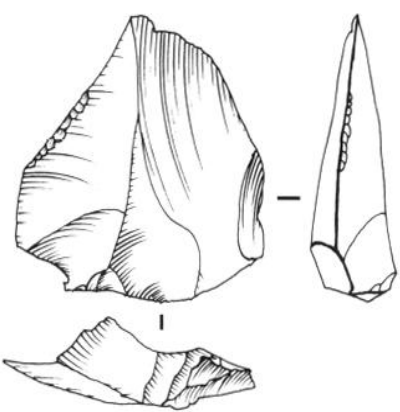

5

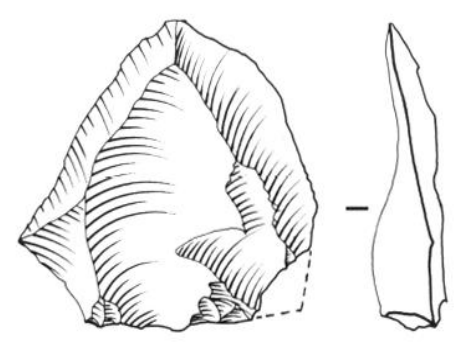

I

Fese

9

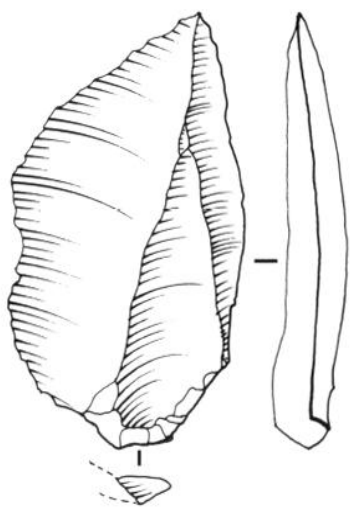

13
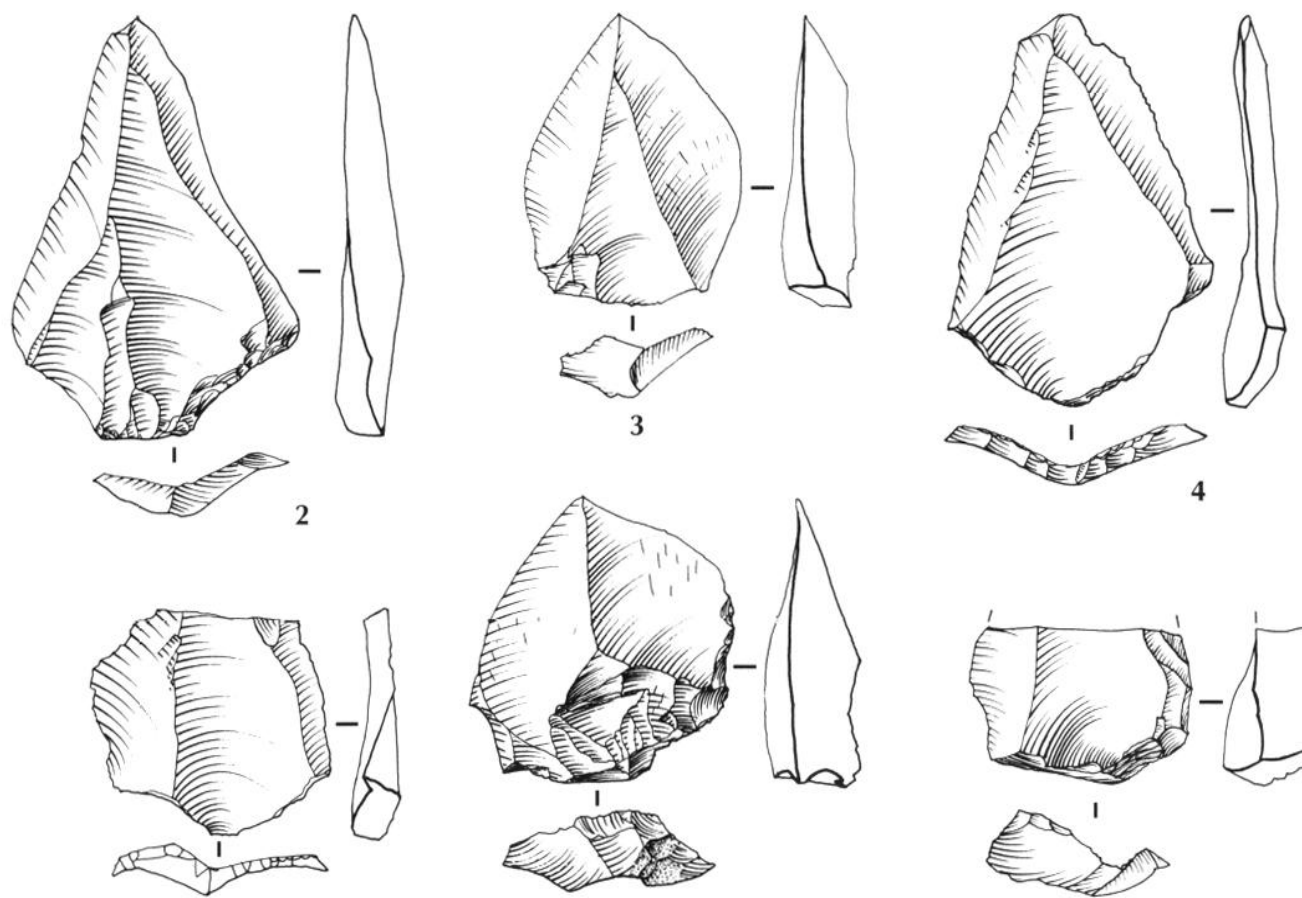

7

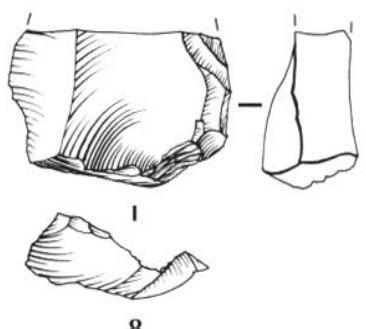

6
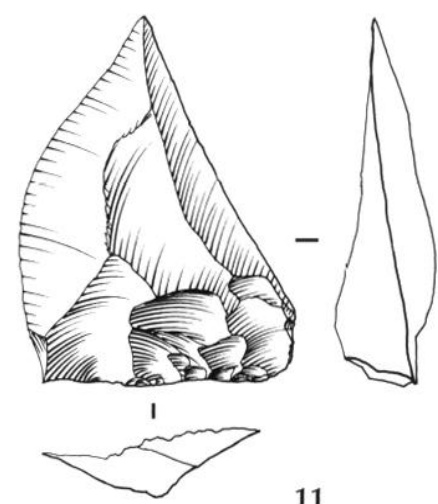

11
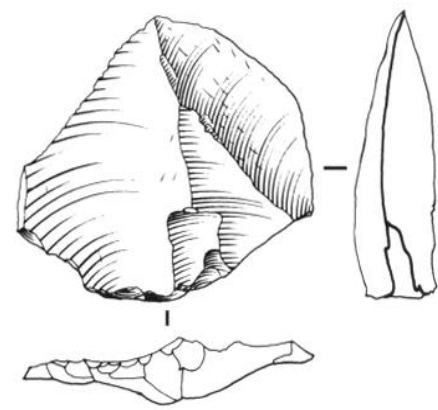

12

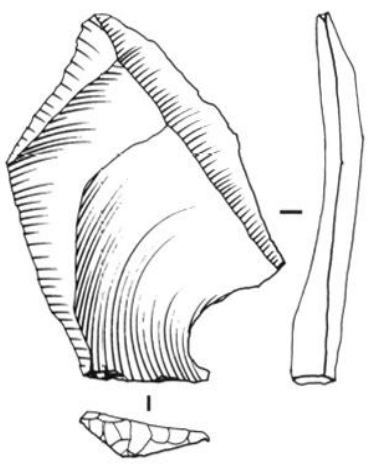

14

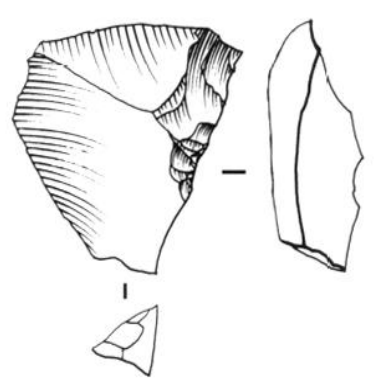

15

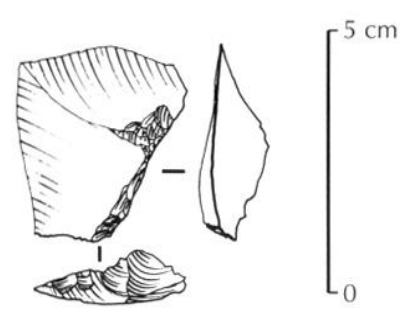

16

Fig. 65 - Pointes Levallois et pseudo-Levallois : 1-9, pointes Levallois en silex tertiaire; 10, pointe Levallois en silex secondaire; 11-14, pointes Levallois en silex tertiaire; 15, pointe pseudo-Levallois en silex secondaire; 16, pointe pseudo-Levallois en silex tertiaire (dessin P. Raymond). 

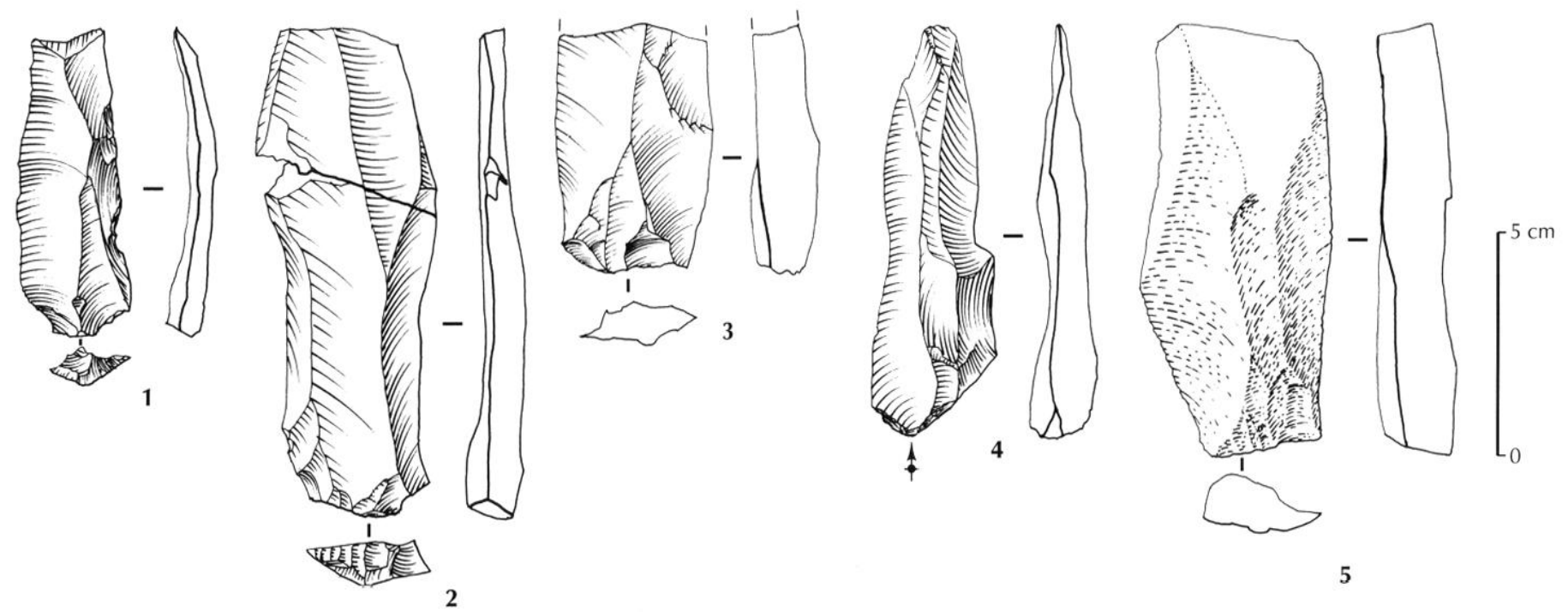

5

Fig. 66 - Lames : I, 4, lames en silex secondaire; 2, 3, lames en silex tertiaire; 5, lame en grès (dessin P. Raymond).

Tabl. XIV - Décompte des différents types d'outils de la série N2 du secteur 1 du Petit Saule à Villiers-Adam.

\begin{tabular}{|l|c|}
\hline \multicolumn{1}{|c|}{ Types d'outils } & Nombre \\
\hline Racloirs simples convexes & 17 \\
\hline Racloirs simples droits & 13 \\
\hline Racloirs doubles convergents & 4 \\
\hline Racloirs déjetés & 4 \\
\hline Racloirs transversaux & 3 \\
\hline Pointes moustériennes & 4 \\
\hline Grattoirs & 2 \\
\hline Lames à dos abattu & 1 \\
\hline Denticulés & 3 \\
\hline Encoches & 2 \\
\hline Éclats retouchés & 9 \\
\hline Total & $\mathbf{6 2}$ \\
\hline
\end{tabular}

Les racloirs, au nombre de 41, dominent largement l'outillage et sont réalisés sur silex tertiaire (28), secondaire (12) et sur grès (1). Les racloirs simples convexes sont les mieux représentés $(n=17)$. Douze sont en silex tertiaire et cinq en silex crétacé. Onze exemplaires portent une retouche abrupte assez écailleuse, dont cinq sur supports assez laminaires (fig. 68). Deux outils de ce type présentent une morphologie particulière. Ils sont aménagés sur éclat épais et allongé, présentant un dos de débitage avec de profonds contre-bulbes opposé à la partie retouchée. La retouche est abrupte et écailleuse (fig. $69, \mathrm{n}^{\text {os }} 5$ et 6 ).
La face ventrale de deux racloirs simples convexes a été amincie par plusieurs enlèvements qui ont, dans un cas, ôté le bulbe. L'un de ces exemplaires porte sur le côté opposé une retouche inverse (fig. 68, $\mathrm{n}^{\circ} 2$ ). Trois racloirs simples convexes présentent une retouche moins envahissante, très abrupte sur un éclat Levallois, semioblique sur un éclat débordant et un éclat Levallois. Le quatrième racloir simple convexe est réalisé par retouche abrupte sur une pointe Levallois dont la pointe a été cassée. Sur la face rendue visible par la cassure se trouvent des traces de chauffe.

Les racloirs simples droits $(n=13)$ sont réalisés sur supports variés, en silex tertiaire (10), secondaire (2) et en grès (1). Deux exemplaires réalisés sur un éclat Levallois, une pointe Levallois et une lame sont aménagés par une retouche marginale continue (fig. 70, $\mathrm{n}^{\circ} 4$ ). Quatre racloirs simples droits portent une retouche semi-abrupte plus envahissante sur toute la longueur du bord. Deux d'entre eux sont aménagés sur éclat de gel. Deux racloirs simples droits sont réalisés sur éclats corticaux par une retouche abrupte assez écailleuse. Trois racloirs simples droits ont été façonnés sur éclat Levallois (1) ou sur lame (2) par une courte retouche oblique. Un racloir simple droit a été aménagé par retouche oblique assez écailleuse sur un éclat de grès. Un racloir simple droit est aménagé par retouche assez rasante sur la face ventrale d'un éclat cortical.

Trois racloirs transversaux ont été réalisés sur éclats courts et larges. L'un est en silex secondaire, les deux 


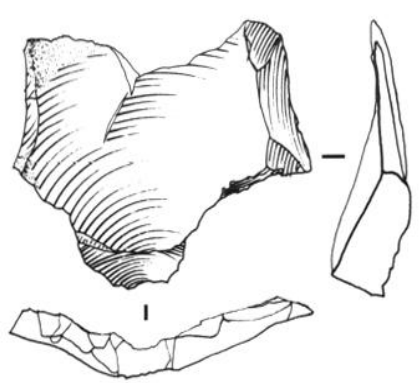

1

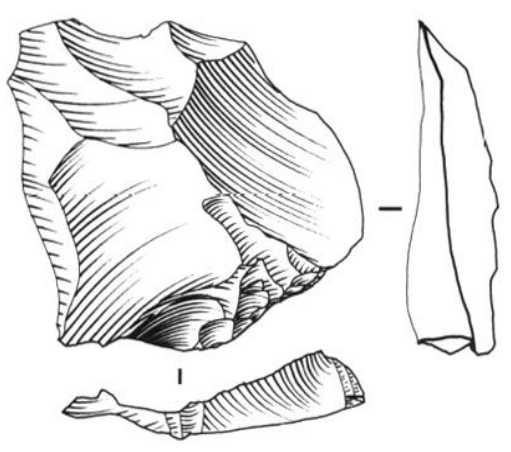

4

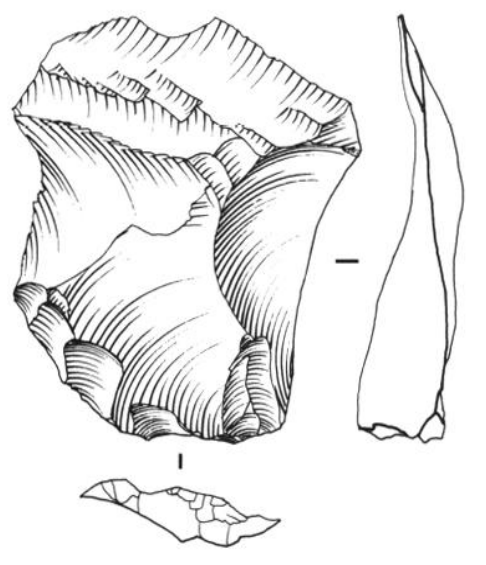

7

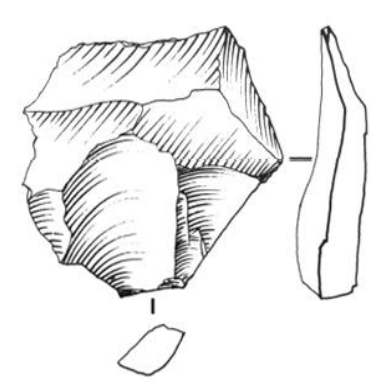

2
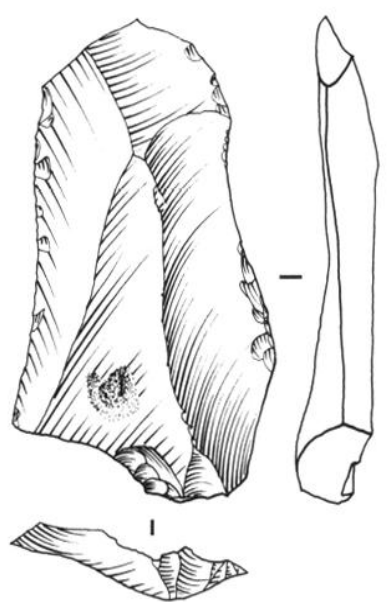

5

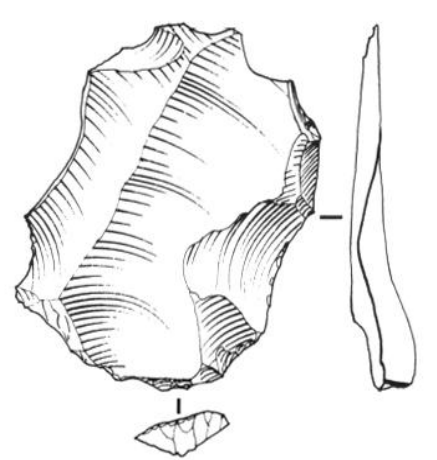

8

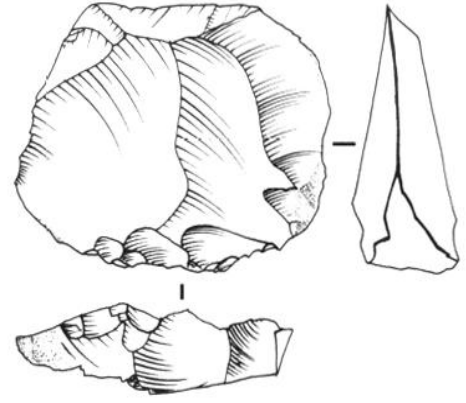

3

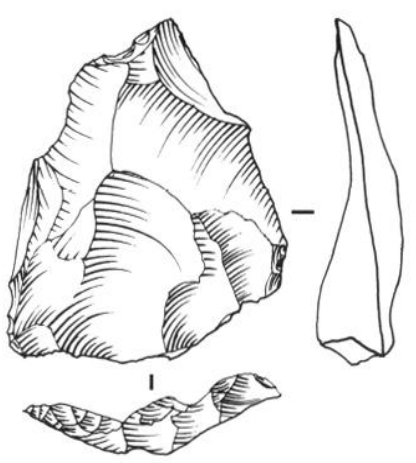

6

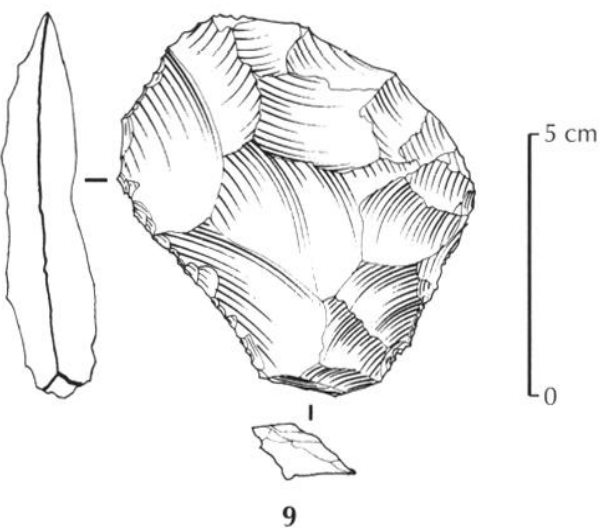

Fig. 67 - Éclats Levallois : 1-3, 5, 8, 9, éclats Levallois en silex tertiaire; 4, 6, 7, éclats Levallois en silex tertiaire (dessin P. Raymond).

autres en silex tertiaire. Sur le premier, la retouche est marginale et continue. Sur le second, elle est abrupte et écailleuse. Sur le troisième, elle est semi-écailleuse.

Les racloirs doubles convergents sont au nombre de quatre. Deux sont réalisés sur éclat Levallois de belle facture, par une retouche semi-oblique peu envahissante (fig. $70, \mathrm{n}^{\circ} 5$ ). Le troisième est réalisé par retouches semiobliques un peu plus envahissantes. La pointe est particulièrement soignée ; la retouche devient plus marginale sur les bords. Cet artefact a été cassé en deux, et chacun des fragments porte un enlèvement postérieur à la cassure (fig. $71, \mathrm{n}^{\circ} 3$ ). Un quatrième racloir double présente une morphologie particulière (fig. $69, n^{\circ} 7$ ). Il a été réalisé sur un éclat de forme irrégulière portant un dos constitué par le négatif d'un enlèvement précédent. Le racloir est désaxé et formé par la convergence de deux bords droits. Plusieurs enlèvements postérieurs au détachement sont visibles sur la face dorsale et ventrale de l'éclat. 


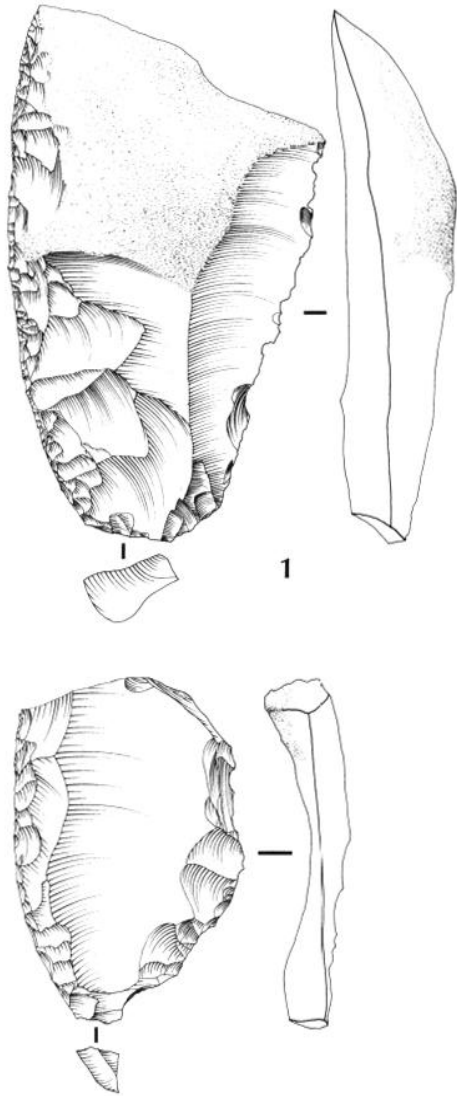

4

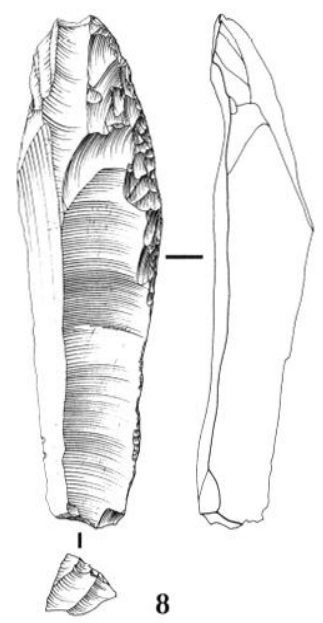

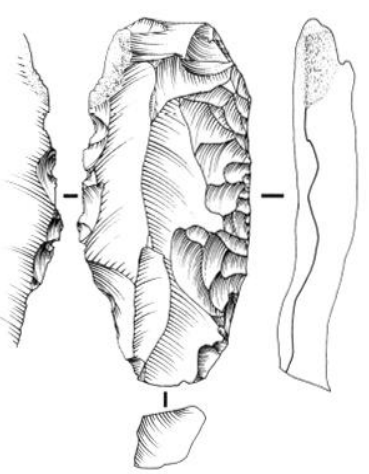

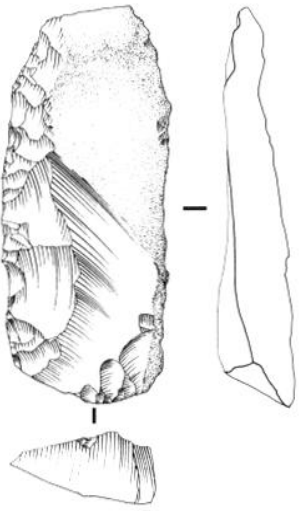

5

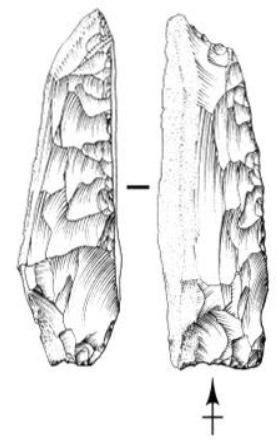

9

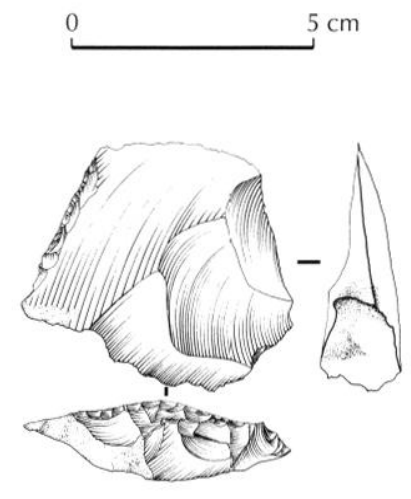

10
2
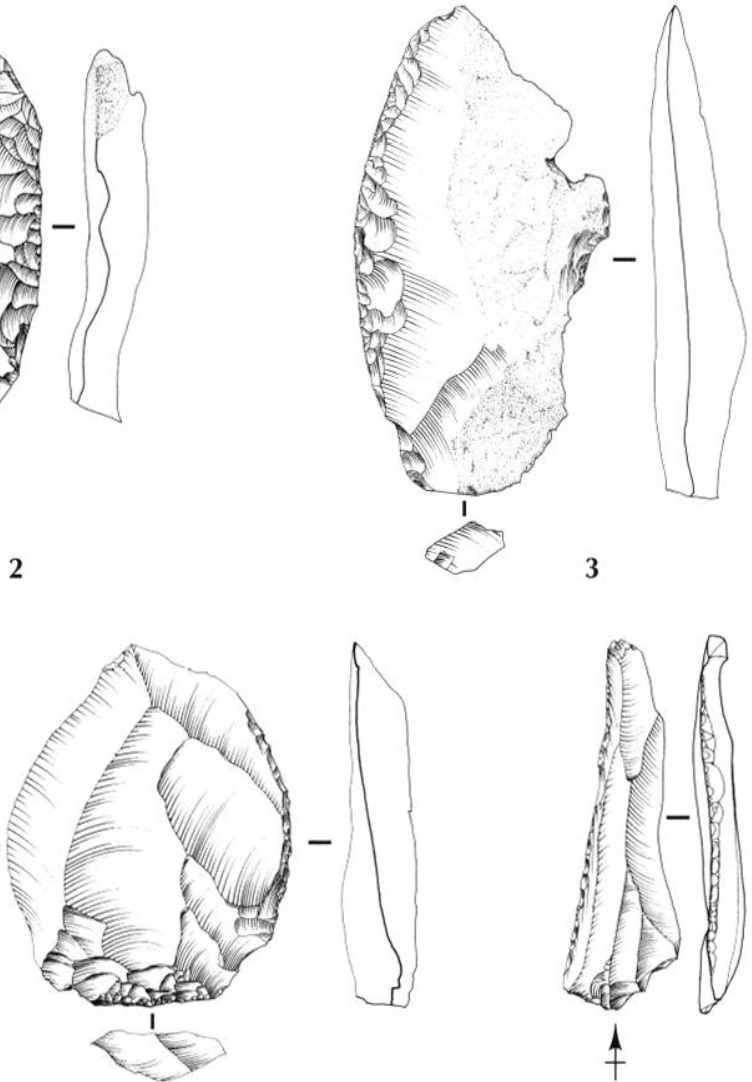

6
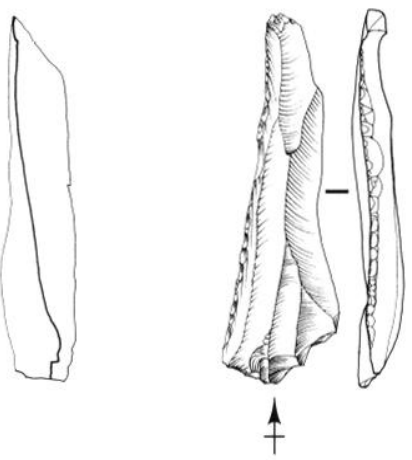

Fig. 68 - Racloirs : 1-3, racloirs simples convexes en silex secondaire; 4, racloir simple convexe en silex tertiaire; 5 , racloir simple convexe en silex secondaire; 6, racloir simple convexe en silex tertiaire; 7, lame à dos abattu en silex secondaire; 8, racloir simple convexe en silex tertiaire; 9-11, racloirs simples droits en silex tertiaire (dessin P. Raymond).

Fig. 69 - Racloirs : 1, racloir simple convexe en silex tertiaire; 2, racloir déjeté en silex tertiaire; 3, racloir déjeté en silex tertiaire, avec amincissement de la face inférieure; 4, racloir transversal en silex tertiaire; 5, 6, racloirs simples convexes en silex tertiaire; 7 , racloir double en silex secondaire; 8, racloir simple convexe en silex tertiaire, avec amincissement de la face inférieure (dessin P. Raymond). 

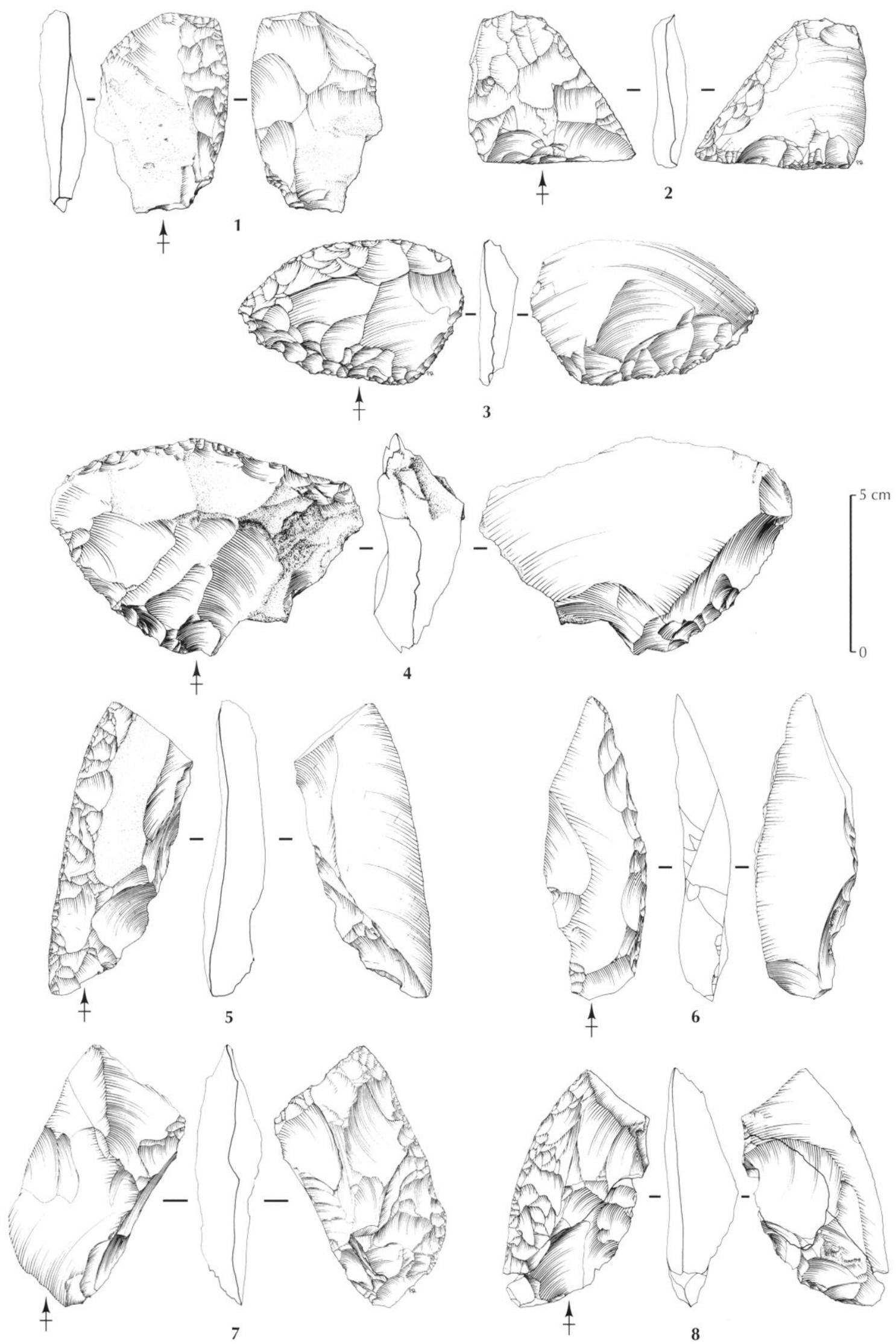


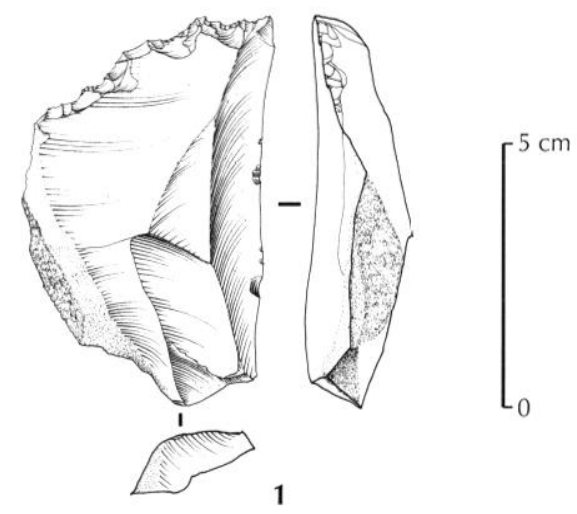

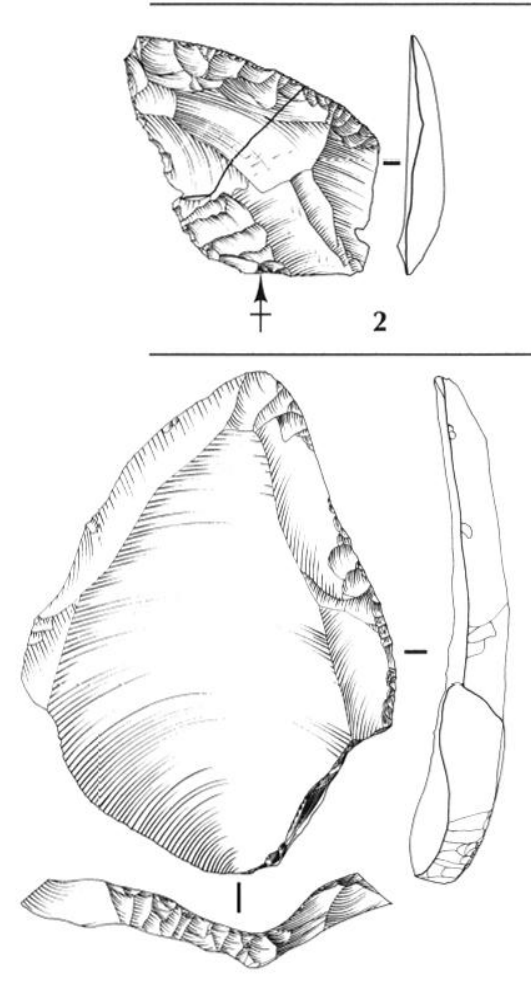

4
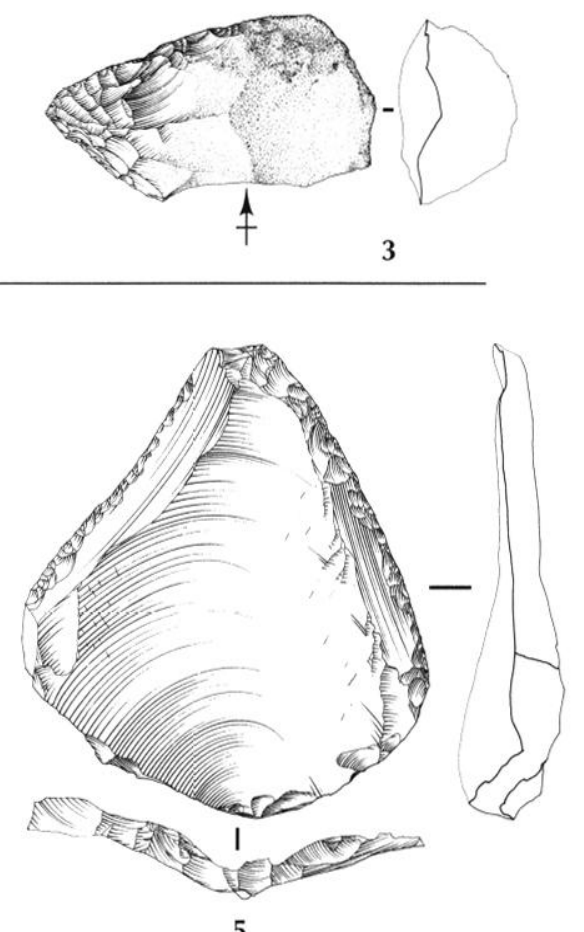

5

Fig. 70 - Outillage retouché: 1, denticulé en silex tertiaire; 2, 3, racloirs déjetés en silex tertiaire; 4, racloir simple droit en silex secondaire; 5 , racloir double en silex secondaire (dessin P. Raymond).

Quatre racloirs déjetés présentent une morphologie assez comparable. L'axe morphologique de l'outil, formé par les deux bords retouchés et convergents, est fortement désaxé par rapport au sens de débitage du support, qui est plus large que long (fig. $69, \mathrm{n}^{\text {os }} 2$ et 3 ). La retouche est semi-oblique dans trois cas, abrupte et écailleuse pour le quatrième. Aucune de ces pièces ne présentent de talon. La première a été amincie sur la face ventrale par de grands enlèvements envahissants; la pointe formée par la convergence des deux bords est de facture très soignée (fig. $69, \mathrm{n}^{\circ} 3$ ). Le second racloir déjeté est réalisé sur la face ventrale d'un éclat. L'extrémité formée par la rencontre des deux bords retouchés est à nouveau très soignée (fig. $69, \mathrm{n}^{\circ} 2$ ). Le troisième est aménagé par une courte retouche sur un petit éclat, le quatrième par retouche abrupte sur un éclat cortical épais (fig. $70, \mathrm{n}^{\mathrm{os}} 2$ et 3 ).

Les pointes moustériennes sont au nombre de quatre. Trois pointes ont été réalisées en silex tertiaire. Deux sont entières. La première, réalisée sur pointe Levallois, est légèrement désaxée vers la gauche et est aménagée par retouches abruptes et écailleuses. Le support de la 

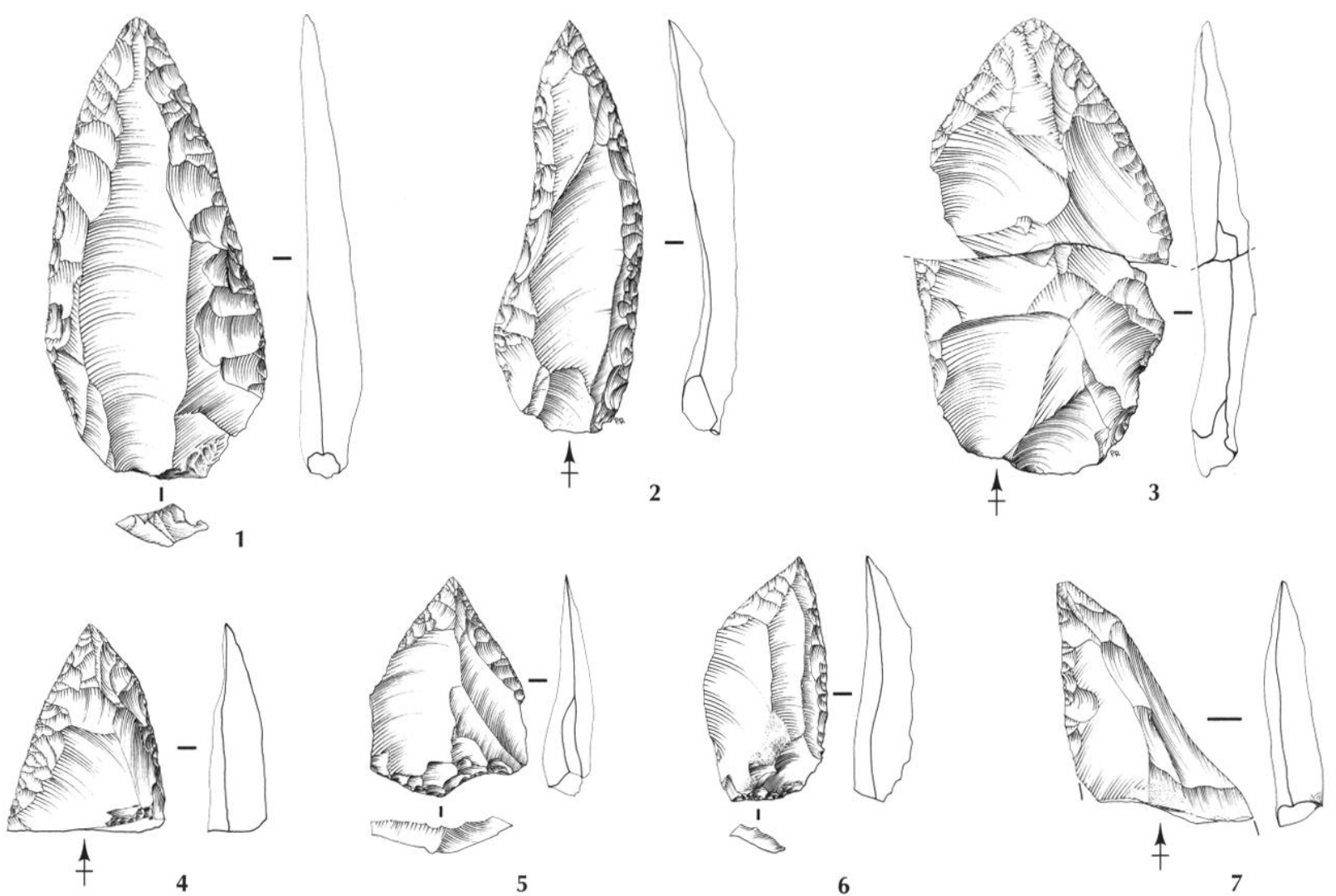

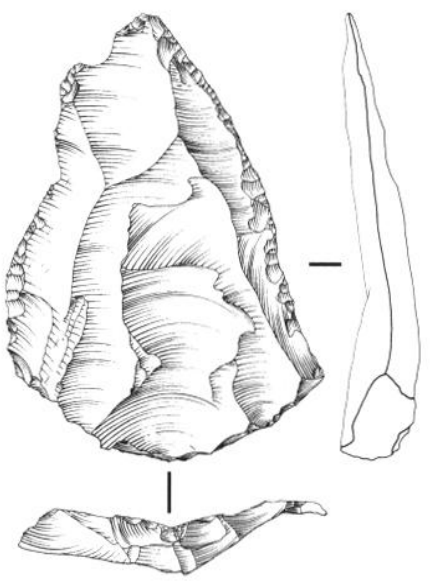

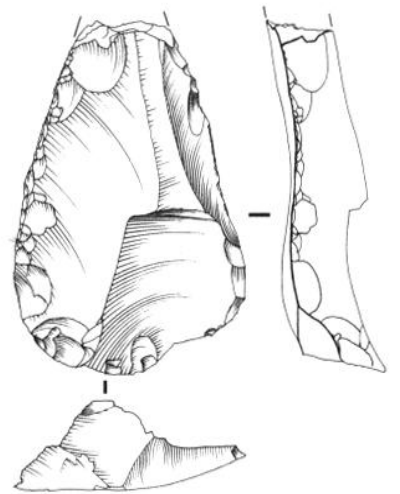
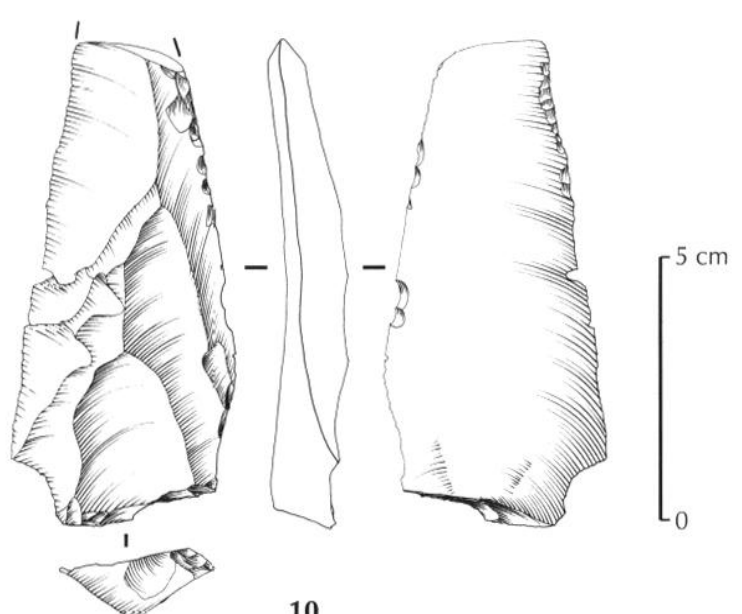

10

Fig. 71 - Outillage retouché: 1, 2, pointes moustériennes en silex tertiaire; 3, racloir double convergent brisé en silex secondaire; 4, pointe moustérienne en silex tertiaire; 5 , pointe moustérienne en silex secondaire; 6 , racloir double convergent en silex tertiaire; 7, racloir simple droit en silex tertiaire; 8 , racloir double en silex secondaire; 9, racloir double en silex tertiaire; 10, racloir simple droit (dessin P. Raymond).

seconde est un éclat Levallois appointé par retouches obliques assez envahissantes (fig. $71, \mathrm{n}^{\text {os }} 1$ et 2 ). Dans les deux cas, la retouche est continue sur les deux bords, de la base jusqu'à la pointe. La troisième pointe, de cassure ancienne, est formée par la convergence du bord droit convexe et du bord gauche rectiligne. La retouche est à nouveau abrupte et écailleuse. Une quatrième pointe moustérienne, en silex secondaire, a été réalisée sur une pointe Levallois par une retouche semi-oblique qui n'affecte que la partie distale du support.

Deux grattoirs en silex tertiaire ne présentent qu'un front partiel. Le premier est réalisé en bout de lame par 
une retouche abrupte, dans la partie sous-corticale du support. Le second est aménagé sur éclat cortical par retouche abrupte.

Une lame en silex secondaire provenant d'un débitage bipolaire porte le seul dos aménagé sur toute la longueur par une retouche très abrupte (fig. $68, \mathrm{n}^{\circ} 7$ ). À l'instar du racloir sur pointe Levallois décrit plus haut, l'extrémité de cette lame est légèrement brisée et de petites craquelures dues au feu sont visibles sur la partie distale. La présence de cet artefact est significative, le débitage laminaire, de conception volumétrique (Révillion, 1994), n'étant pas représenté dans la série lithique de ce secteur, si ce n'est par cet artefact. Elle permet d'établir une comparaison avec les industries à lames du nord de la France (Seclin: Tuffreau $e t$ al., 1994; Riencourt-les-Bapaume: Tuffreau dir., 1993 ; Bettencourt-Saint-Ouen : Locht dir., 2002), au sein desquelles les outils de type Paléolithique supérieur sont systématiquement réalisés sur support laminaire lorsque ce type de production est identifié.

Deux encoches en silex tertiaire ont été retrouvées. La première, peu profonde, se trouve sur la partie mésiale d'une petite lame à dos cortical. La seconde, assez profonde, a été aménagée sur la face ventrale d'une grande pointe pseudo-Levallois.

Les denticulés ne sont qu'au nombre de trois. Le seul exemplaire en silex tertiaire est réalisé sur éclat cortical par la juxtaposition de quatre petites encoches peu profondes, aménagées par de petites retouches abruptes (fig. $70, \mathrm{n}^{\circ} 1$ ). Les deux autres, en silex secondaire, ont été réalisés sur la partie distale d'éclats corticaux par la juxtaposition de plusieurs encoches.

Un éclat Levallois porte sur l'extrémité distale d'un bord une courte retouche oblique. Six éclats, deux cassons et un éclat de gel, ainsi qu'une lame en grès, portent une courte retouche marginale qui n'affecte qu'une petite partie du bord.

\section{Analyse fonctionnelle}

Quarante-cing artefacts en silex tertiaire et vingt-six en silex secondaire ont fait l'objet d'une analyse fonctionnelle et ont été examinés au microscope optique à fort grossissement (de 100 à 500 fois) par Jean-Paul Caspar (Katholieke Universiteit te Leuven, Belgique). Leur état d'altération n'a pas permis de déceler de traces d'usage.

\section{LA SÉRIE LITHIQUE DU SECTEUR 2}

Le secteur 2 a livré 154 artefacts. À l'époque de l'occupation préhistorique, le cailloutis sous-jacent aux formations superficielles affleurait à cet endroit du versant. Malgré l'accès facile à ce matériau, les préhistoriques ont privilégié, dans ce secteur, la taille du silex crétacé (voir p. 72). Quelques remontages ont été réalisés sur matériau secondaire et tertiaire. Seuls trois raccords de cassures anciennes, intervenues lors de la taille, ont été effectués.

La lecture diacritique des nucléus est difficile en raison de leur petite taille et de la mauvaise qualité de la matière première tertiaire. Celle des nucléus en silex crétacé ne l'est pas moins car, de meilleure qualité, ils ont subi un phénomène de réduction très poussé. L'identification des différents schémas a été faite par comparaison avec ceux déterminés dans le secteur 1 , dans lequel la documentation technologique est abondante et de meilleure qualité.

\section{Chaîne opératoire à pointes}

\section{Schéma unipolaire convergent (3 nucléus)}

Le débitage unipolaire convergent est représenté par trois nucléus; deux sont en silex secondaire et le troisième en silex tertiaire. Le premier, de petite taille, est réalisé sur bloc (fig. $72, n^{\circ} 4$ ). Le plan de frappe est facetté et installé sur la partie la plus large du rognon. L'entretien des convexités est assuré par le détachement d'éclats ou de lames à dos cortical. Le second est réalisé sur un éclat. Le plan de frappe est sommairement facetté, et deux enlèvements de directions convergentes ont été détachés. Un troisième nucléus unipolaire convergent a été réalisé sur un éclat de gel de silex tertiaire. À partir d'un plan de frappe sommairement préparé, trois enlèvements de directions convergentes ont été détachés.

\section{Chaîne opératoire à éclats}

Schéma opératoire Levallois récurrent bipolaire (2 nucléus)

Deux nucléus Levallois en silex crétacé ont été débités selon une modalité récurrente bipolaire. L'exploitation de ces blocs est assez poussée. Les convexités sont aménagées par de petits enlèvements de direction centripète. La surface de plan de frappe a été soigneusement préparée. Les enlèvements ont été détachés à partir de 
Fig. 72 - Industrie lithique du secteur $2: 1$, racloir simple convexe en silex tertiaire, avec amincissement de la face inférieure ; 2, pointe moustérienne en silex secondaire; 3, nucléus à lames semi-tournant en silex secondaire; 4 , nucléus unipolaire. convergent en silex secondaire (dessin P. Raymond).
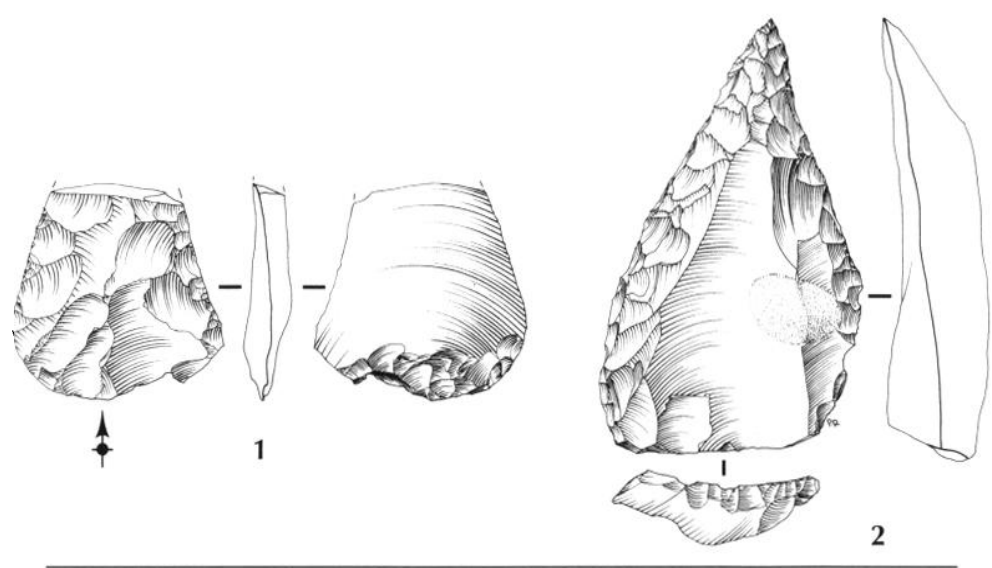

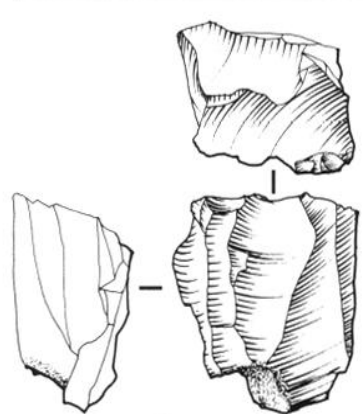

3

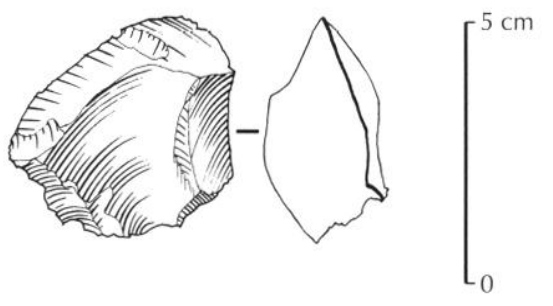

4 ces deux plans de frappe utilisés alternativement. Les deux derniers enlèvements Levallois ont laissé de profonds contre-bulbes qui ont empêché toute poursuite de débitage organisé.

Schéma opératoire Levallois récurrent centripète (1 nucléus)

Ce schéma ne concerne qu'un seul nucléus en silex tertiaire, assez désilicifié, de lecture difficile. Il présente deux surfaces opposées, hiérarchisées, l'une servant de plan de frappe pour le débitage de l'autre. L'exploitation de cette dernière se fait à partir d'un plan de frappe périphérique.

\section{Schéma opératoire bipolaire (2 nucléus)}

Deux nucléus en silex secondaire ont subi un phénomène de réduction très poussé. Deux plans de frappe, bien préparés, servent au détachement des éclats. La surface de production ne porte pas d'aménagement de convexités, mais il pourrait s'agir de nucléus Levallois exploités jusqu'à exhaustion.

\section{Schéma unipolaire parallèle (1 nucléus)}

Un bloc de petite dimension a été exploité selon une modalité unipolaire parallèle. Vu la qualité du matériau (silex tertiaire avec géode), le débitage s'est arrêté très rapidement. Un nucléus informe a subi l'action du gel et a permis le détachement d'à peine deux éclats.

\section{Chaîne opératoire à lames}

\section{Schéma opératoire semi-tournant (1 nucléus)}

Un nucléus en silex crétacé a produit des lames selon une mode de débitage semi-tournant (fig. $72, \mathrm{n}^{\circ} 3$ ). L'état final du nucléus ne permet pas de déceler le processus d'initialisation du débitage. Il a subi un important phénomène de réduction. Le débitage a été conduit à partir d'un seul plan de frappe. L'état final du nucléus ne laisse voir que des négatifs d'enlèvements laminaires parallèles.

La présence de ce nucléus revêt une signification particulière sur ce gisement. Associé à des chaînes opératoires orientées vers la production de lames et de pointes, il démontre l'étendue des connaissances technologiques des artisans du Paléolithique moyen. Il confirme également que la production laminaire est sans doute connue par les tailleurs de Villiers-Adam, mais qu'elle est sous-représentée par rapport aux autres systèmes productifs. Ce fait avait déjà été pressenti dans 
le secteur 1 en considérant les supports laminaires, dont l'un a été transformé en pièce à dos abattu (fig. $68, \mathrm{n}^{\circ} 7$ ).

\section{Les produits}

Les pointes, au nombre de 6 , ont été produites à partir de nucléus unipolaires convergents. Deux sont en silex tertiaire, trois en silex secondaire et une seule en grès à grain très fin.

Seuls 3 éclats Levallois sont en silex tertiaire ; 11 sont en silex secondaire. Ils ont été produits par le biais d'un débitage Levallois récurrent, bipolaire ou centripète, comme le laisse supposer la direction des négatifs d'enlèvements présents sur leur face dorsale.

Vingt-trois lames ont été retrouvées dans ce secteur ; 8 sont en silex tertiaire et 15 en silex secondaire. Elles peuvent provenir de nucléus unipolaires convergents comme dans le secteur 1, mais aussi de nucléus à lames semi-tournants, dont un exemplaire a été retrouvé dans cette zone.

\section{L'outillage}

Cinq outils en silex secondaire ont été retrouvés dans le secteur 2, à savoir deux racloirs simples droits, un racloir double, une pointe moustérienne et un éclat retouché. L'un des deux racloirs simples est réalisé sur un éclat cortical par une retouche semi-oblique continue. Le second est aménagé par une retouche très oblique sur la partie proximale d'un éclat cortical, le reste du tranchant est brut de toute retouche. Le racloir double a été aménagé par une retouche semi-oblique, et la base amincie sur la face ventrale par une retouche du même type (fig. $72, \mathrm{n}^{\circ} 1$ ). La pointe moustérienne est réalisée sur une épaisse pointe Levallois par retouches écailleuses abruptes (fig. $72, \mathrm{n}^{\circ}$ 2). Le bord droit d'une petite pointe Levallois a été très légèrement retouchẻ sur moins d'un centimètre. Deux racloirs simples, l'un droit et l'autre convexe, sont réalisés sur éclats corticaux en silex tertiaire par une retouche semi-abrupte. Un racloir simple droit de belle facture a été réalisé sur une pointe Levallois en grès très fin. La retouche est oblique (fig. $73, \mathrm{n}^{\circ} 3$ ).

\section{LA SÉRIE LITHIQUE DU SECTEUR 3}

Cette série est limitée et ne contient que 55 artefacts. D'après l'analyse des nucléus, elle est orientée vers la pro- duction d'éclats. Un seul raccord de cassure ancienne a été réalisé sur cet ensemble.

\section{Chaîne opératoire à éclats}

Schéma opératoire Levallois récurrent centripète (2 nucléus)

Deux nucléus ont été exploités selon ce système. Ils sont de petites dimensions. L'un des deux est réalisé sur un éclat de gel (fig. $73, \mathrm{n}^{\circ} 5$ ). Un plan de frappe périphérique, assez peu soigné, est installé sur la surface inférieure. Le débitage de la seconde surface a été poussé au maximum. L'exploitation se termine par l'obtention de petits éclats, rebroussés en raison des faibles convexités de la surface productive.

\section{Schéma opératoire unipolaire convergent (1 nucléus)}

Un seul nucléus de ce type, en silex secondaire, a été exploité jusqu'à exhaustion. Il présente une dernière surface de débitage, plus large que longue, elle-même débitée à partir d'un plan de frappe vraisemblablement constitué par une ancienne surface productive.

Schéma opératoire bipolaire (1 nucléus)

Un petit nucléus a été débité à partir de deux plans de frappe opposés, réalisés par l'enlèvement d'un seul éclat cortical. L'axe de percussion est assez oblique par rapport au plan d'articulation des deux surfaces. Le dernier nucléus de la série est un éclat de gel duquel un seul enlèvement a été détaché.

\section{Les produits}

Trois éclats Levallois ont été retrouvés dans ce secteur. Deux sont en silex secondaire, et le troisième en silex tertiaire. Trois lames sont aussi présentes, deux en silex crétacé et une en grès. Deux pointes en matériau tertiaire complètent ce décompte réduit.

\section{L'outillage}

Cinq outils retouchés ont été retrouvés. Le seul outil en silex secondaire est un racloir simple droit aménagé sur un éclat cortical par une courte retouche marginale très abrupte.

Un racloir simple convexe est aménagé sur un éclat cortical par une retouche régulière, oblique et légèrement écailleuse (fig. $73, \mathrm{n}^{\circ} 1$ ). Un racloir double convexe 


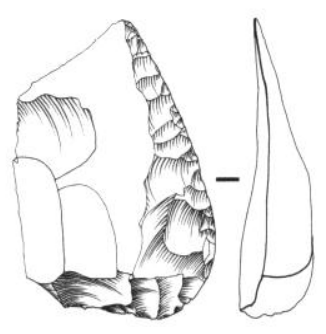

1

4

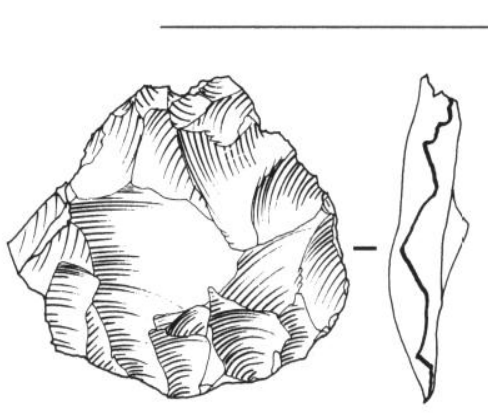

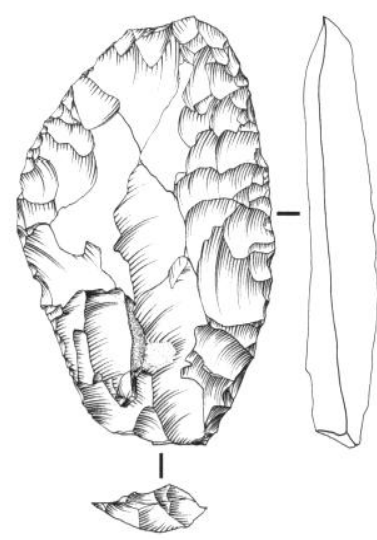

2

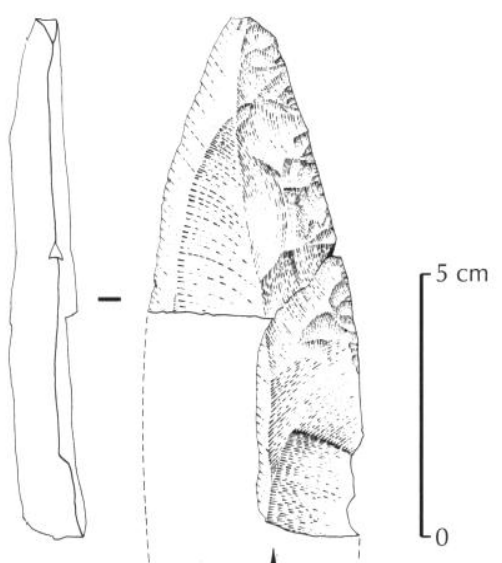

3 千

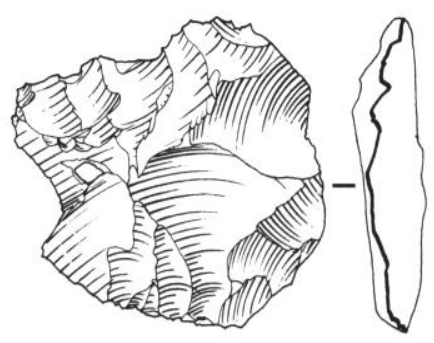

5

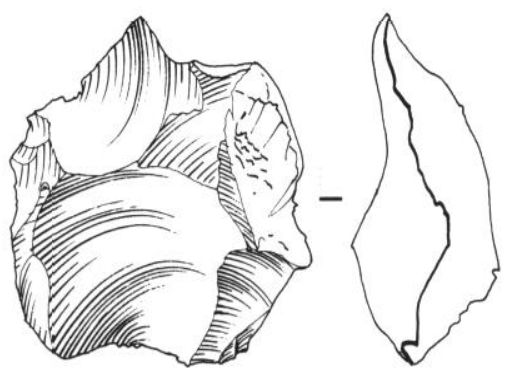

6

Fig. 73 - Industrie lithique des secteurs 2 et 3:1, racloir simple convexe en silex tertiaire (secteur 3) : 2, racloir double en silex tertiaire (secteur 3) ; 3, pointe moustérienne en grès (secteur 2) ; 4, 5, nucléus Levallois récurrents centripète (secteur 3) ; 6, nucléus unipolaire convergent en silex secondaire (secteur 3) (dessin P. Raymond).

a été réalisé sur un éclat Levallois par le biais d'une retouche semi-oblique envahissante (fig. 73 , $\mathrm{n}^{\circ}$ 2). Une pointe Levallois, dont la pointe a été cassée anciennement, porte sur le côté droit une courte retouche semi-oblique. Un enlèvement burinant a été détaché sur l'arête naturelle d'un éclat de gel. Dans ce cas, il est délicat de faire la différence entre la volonté de réaliser un burin et celle de transformer cet éclat en nucléus.

\section{INTERPRÉTATION}

\section{Le secteur 1}

Malgré la présence de trois matériaux aux qualités variables, il n'y a pas d'association particulière entre type de matériau et chaîne opératoire. La différence se situe au niveau de la gestion des blocs, puisque les rognons de silex secondaire ont subi un phénomène de réduction plus poussé que ceux en silex tertiaire.
Deux chaînes opératoires ont été distinguées et sont orientées vers la production respective d'éclats et de pointes (fig. 74).

Cette dernière chaîne opératoire est dominante et est surtout représentée par un schéma de débitage unipolaire convergent (84 nucléus) (tabl. XV). Ce schéma de production permet l'obtention rapide de pointes tout en limitant l'investissement technologique grâce à l'utilisation adéquate des convexités naturelles du bloc. La plupart du temps, une seule surface s'inscrit dans le volume du bloc. Un algorithme de base, qui consiste en la production de lames à dos cortical établissant les convexités latérales et du détachement d'une pointe Levallois, peut être répété plusieurs fois de suite sur la surface productive. Il peut aussi être déplacé sur une partie adéquate du bloc, la première surface productive sert alors de plan de frappe pour la seconde. La production s'effectue ainsi par surfaces alternées. Chacune des surfaces considérée individuellement se rapproche d'un schéma opératoire Levallois. Toutefois, l'état final du 


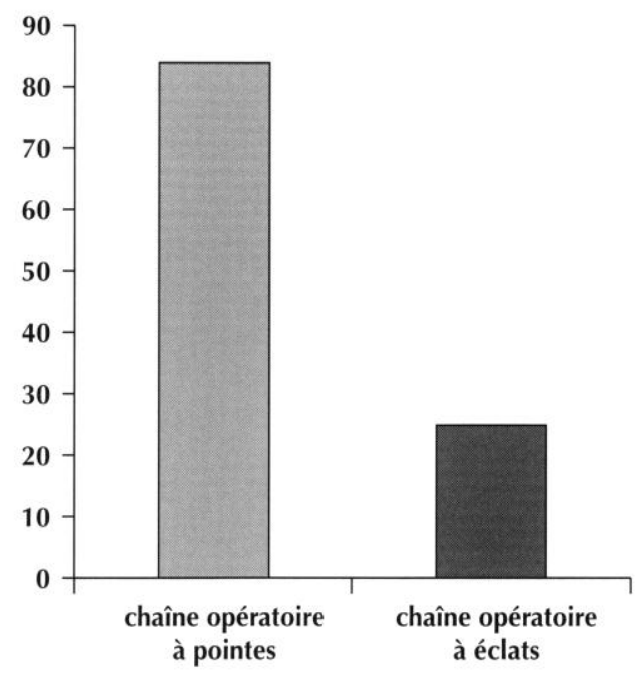

Fig. 74 - Histogramme des chaînes opératoires.

bloc résiduel et l'examen diacritique des remontages incitent plutôt à classer ces nucléus dans la classe des nucléus à morphologie variable (volume non homothétique), plutôt que dans celle des nucléus Levallois dont la structure volumétrique reste figée (Boëda, 1997). Ce système de production n'a été identifié à l'heure actuelle dans le nord-ouest de la France que sur le gisement de Bettencourt-Saint-Ouen, Somme (Locht dir., 2002), où il coexiste avec d'autres chaînes opératoires (laminaire, Levallois stricto sensu...).

En ce qui concerne la production des éclats, le schéma opératoire le plus fréquent est unipolaire parallèle (25 nucléus), parfois proche de son équivalent destiné à la production de pointes. Cependant, les nucléus n'ont été exploités qu'aux dépens d'une seule face productive. Cette méthode de production coexiste avec les modalités Levallois récurrente centripète et bipolaire (respectivement 10 et 3 exemplaires).

Une production de lames selon un débitage semitournant ou tournant (Révillion, 1994) est peut-être représentée par quelques artefacts provenant d'un débitage bipolaire (fig. 66, $\mathrm{n}^{\circ} 4$ ). Les nucléus de ce type sont absents. Néammoins, la présence d'une lame à dos abattu de belle facture, en silex secondaire exogène, pourrait laisser envisager l'hypothèse d'une importation d'artefacts produits en dehors de l'aire de fouille, voire sur un autre gisement. En effet, sur d'autres sites du début de la dernière glaciation, ce type de débitage existe également, en proportion minoritaire par rapport aux autres chaînes opératoires, comme c'est le cas à
Tabl. XV - Décompte des différents types de nucléus de la série lithique N2 du secteur 1 du Petit Saule à Villiers-Adam.

\begin{tabular}{|l|r|r|}
\hline \multicolumn{1}{|c|}{ Types de nucléus } & Nombre & \multicolumn{1}{c|}{$\%$} \\
\hline Nucléus unipolaires convergents & 84 & 49,42 \\
\hline Nucléus unipolaires parallèles & 25 & 14,71 \\
\hline Nucléus bipolaires à éclats & 9 & 5,29 \\
\hline Nucléus Levallois récurrents centripètes & 11 & 6,47 \\
\hline Nucléus Levallois récurrents bipolaires & 3 & 1,76 \\
\hline Nucléus informes & 38 & 22,35 \\
\hline Total & $\mathbf{1 7 0}$ & $\mathbf{1 0 0 , 0 0}$ \\
\hline
\end{tabular}

Bettencourt-Saint-Ouen, niveau Nl (Locht dir., 2002), à Gouy-Saint-André, Pas-de-Calais (Depaepe, Deschodt, 2001) ou encore sur les sites de la vallée de la Vanne (Deloze et al., 1994).

Comme dans la plupart des gisements de plein air du Paléolithique moyen qui présentent une densité d'artefacts faible ou moyenne, l'outillage retouché ne représente qu'un faible pourcentage de la série $(2,83 \%)$. Ce faible pourcentage ne dénote toutefois pas par rapport aux autres séries du Début glaciaire weichsélien du nord de la France. Ainsi, la série N2b du gisement de Bettencourt-Saint-Ouen contient moins de $2 \%$ de pièces retouchées. Il faut à nouveau souligner la probable utilisation des supports bruts (outils a posteriori), et notamment des pointes, dans le cas précis de VilliersAdam.

Les racloirs, surtout simples, sont très largement majoritaires dans cette série du secteur 1 et donnent à cette série un caractère moustérien prononcé. Les outils de type Paléolithique supérieur sont très peu présents, mais réalisés sur support allongé. Le seul dos abattu a été aménagé sur une belle lame de silex provenant d'un débitage bipolaire. Les encoches et les denticulés sont également peu nombreux (fig. 75).

Près de la moitié de l'outillage retouché a été aménagé par des retouches, semi-abruptes ou abruptes, et envahissantes, qui peuvent témoigner du réavivage du tranchant de ces outils. La présence de plusieurs amincissements sur la face ventrale des artefacts retouchés, ainsi que la présence d'enlèvements sur la partie distale de quelques pièces, évoquent la possibilité d'un emmanchement des outils (Locht, Antoine, 2001) (fig. 69, $\mathrm{n}^{\text {os }} 2$, 3 et 8 ). 
Il ne semble pas y avoir de relation entre matériau, système de production et type d'outils. Un soin particulier semble cependant avoir été accordé au choix du support des outils à bords convergents, tels que les racloirs doubles et les pointes moustériennes.

\section{Le secteur 2}

Le nombre restreint d'artefacts du secteur 2 n'autorise pas d'interprétation poussée. La différence majeure réside dans la plus grande utilisation du silex secondaire par rapport au silex tertiaire. La chaîne opératoire à éclats est légèrement dominante dans cette série en regard de la production de pointes (respectivement 5 et 3 nucléus), mais l'on ne peut tirer de conclusions à partir d'un si faible effectif. La présence d'un nucléus à débitage semi-tournant est particulièrement évocatrice puisqu'elle confirme la connaissance de ce type de débitage par les taillleurs de Villiers-Adam. Cette observation vient rejoindre celle effectuée à propos de la série du secteur 1, au sein de laquelle la présence d'une lame à dos abattu évoquait les séries à débitage laminaire du nord de la France (Seclin, Riencourt-les-Bapaume, Bettencourt-Saint-Ouen, etc.).

Si l'on considère ces aspects technologiques ainsi que ceux de l'outillage, la série du secteur 2 ne présente pas de différences fondamentales avec celle du secteur 1 et pourrait être l'œuvre du même groupe culturel.

\section{Le secteur 3}

À l'inverse du secteur 2, le silex tertiaire a été plus utilisé que son homologue crétacé dans ce secteur. En revanche, le profil de la série semble plus orienté vers la production d'éclats que vers celle de pointes. L'échantillon est toutefois réduit et le spectre technotypologique de cet ensemble le rapproche plutôt de celui du secteur 1 .

En considérant l'ensemble de ces observations, et même en l'absence de corrélation stratigraphique, la parenté des industries des trois secteurs semble être une réalité archéologique, ce qui ne suppose pas pour autant leur exacte contemporanéité.

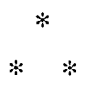

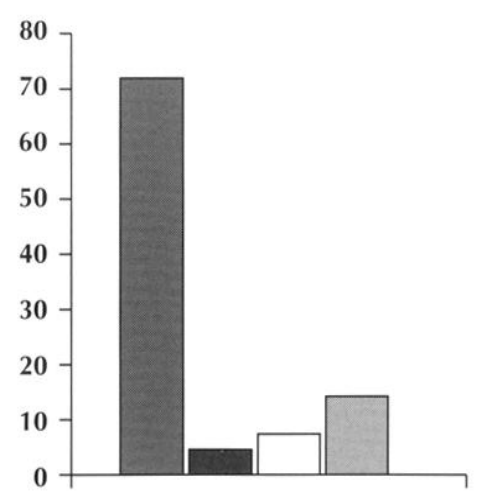

outils du groupe moustérien (racloirs et pointes moustériennes)

outils de type paléolithique supérieur

denticulés et encoches

éclats retouchés

Fig. 75 - Proportion des différents types d'outils du secteur 1.

Le gisement paléolithique moyen du Petit Saule, situé sur un versant en pente douce et exposé au nord-est, est recouvert d'une épaisse séquence limoneuse qui a permis la conservation en place d'un niveau archéologique. Cette situation topographique est courante pour les gisements de plein air de cette période.

Découvert en mars 1996, le gisement a été fouillé d'août à novembre 1996, sur une superficie totale de $3886 \mathrm{~m}^{2}$, répartie en trois secteurs séparés par des zones où le niveau archéologique a été démantelé par érosion. La découverte du gisement paléolithique et des séquences stratigraphiques du Chamesson et de VA-99 constitue un événement majeur pour la connaissance du Pléistocène dans le nord de la France.

D'un point de vue stratigraphique, la séquence de Villiers-Adam est exceptionnelle pour la région Île-deFrance et pour le nord de la France de par l'accumulation loessique de $19 \mathrm{~m}$ d'épaisseur. Ce bilan est exceptionnel, tout particulièrement pour le début de la dernière glaciation, et a révélé un enregistrement pédosédimentaire original, uniquement connu dans le nord de la France sur le site Saint-Sauflieu, Somme (Antoine, 1989) et de Bettencourt-Saint-Ouen, Somme (Antoine, 2002).

L'intérêt de cette coupe stratigraphique a motivé l'intervention de plusieurs spécialistes avec pour objet l'étude pluridisciplinaire de cette séquence pléistocène. Ainsi, études stratigraphiques, sédimentologiques, 
malacologiques, palynologiques, datations TL sur silex chauffés et sur sédiments, et études archéologiques ont permis de déboucher sur une interprétation globale du site paléolithique moyen et de la séquence pléistocène.

Les observations de terrain montrent que le niveau paléolithique principal du Petit Saule se localise au sein de dépôts à dominante colluviale qui correspondent à un bilan pédosédimentaire complexe et peu épais, par comparaison aux enregistrements plus complets de VA-99 et du Chamesson. D'après l'interprétation chronostratigraphique, le niveau archéologique serait contemporain de la fin de l'interstade Brørup/SaintGermain I, ce qui est en accord avec les datations TL sur silex chauffés qui ont livré pour l'occupation préhistorique une estimation moyenne de l'ordre de $110 \pm 11 \mathrm{ka} \mathrm{BP}$.

L'étude du matériel lithique a mis en évidence une industrie orientée vers la production de pointes Levallois selon des schémas opératoires originaux. Le débitage Levallois est représenté surtout par des schémas de production récurrents. Quelques artefacts évoquent un débitage laminaire selon des schémas semi-tournants. L'outillage est constitué d'artefacts transformés essentiellement en racloirs par une retouche semi-abrupte ou abrupte et envahissante.

Le gisement et les coupes pléistocènes de VilliersAdam sont ainsi des jalons importants pour la connais- sance du Paléolithique moyen et du Pléistocène supérieur en Île-de-France, et par extension dans le Nord-Ouest européen, de par le nombre et la valeur des informations qu'ils ont livrées. Ils contribuent à l'élaboration d'un schéma chronostratigraphique de plus en plus précis et à la connaissance du mode de vie des populations humaines au Début Glaciaire weichsélien, aux environs de $105000 \mathrm{BP}$.

\section{Remerciements}

Les auteurs tiennent à remercier le conseil général du Val-d'Oise, qui a financé cette étude, le service archéologique du Val-d'Oise pour son accueil et son soutien logistique et administratif, et la direction départementale de l'Équipement du Val-d'Oise pour l'accès au terrain et aux moyens nécessaires à la réalisation des fouilles. Ils remercient en particulier P. Soullier, C. Toupet, S. Rigomond, S. Pierens, M. Gaultier, V. Lemoine, J. Vasquez et L. Costa (service départemental d'archéologie du Val-d'Oise), D. Obert (univ. Paris VI), M. Patou-Mathis (CNRS), J. Fabre (univ. JulesVerne, Amiens), J.-P. Caspar (Katholieke Universiteit te Leuven, Belgique), A. Dietrich et V. Krier (INRAP) pour leur aide sur le terrain, en laboratoire ou dans les tâches administratives. Ils remercient enfin É. Boëda pour ses conseils avisés lors de la rédaction de cet article. 


\section{BIBLIOGRAPHIE}

ABlin D.

1991 : «Analyse pollinique des dépôts lacustres de Ceyssac, Plio-Pléistocène du Velay (Massif central, France) ". Cahiers de micropaléontologie, $\mathrm{n}^{\circ}$ spécial 6, p. 21-38.

\section{Almond P. C., Tonkin P. J.}

1999 : « Pedogenesis by Upbuilding in an Extreme Leaching and Weathering Environment, and Slow Loess Accretion, South Westland New Zealand ". Geoderma, 92, p. 1-36.

Ameloot-Van der Heijden N.

1996 : " Gisement de Biache-Saint-Vaast (Pas-de-Calais). Les matières premières au Paléolithique moyen ", in La Vie préhistorique. Paris, éd. Faton, p. 144-147.

ANTOINE P.

1989 : « Le complexe de sols de SaintSauflieu (Somme), micromorphologie et stratigraphie d'une coupe type du début Weichsélien " in Tufrreau A. (dir.), Paléolithique et Mésolithique du nord de la France. Lille, univ. des sciences et technologies (Publications du Centre d'études et de recherches préhistoriques, 1), p. 51-59.

1990 : Chronostratigraphie et environnement du Paléolithique du bassin de la Somme. Lille, univ. des sciences et technologies (Publications du Centre d'études et de recherches préhistoriques, 2), $231 \mathrm{p}$.

2002 : " Contexte stratigraphique et paléopédologique: interprétation chronostratigraphique et environnementale ", in LOCHT J.-L (dir.), Bettencourt-Saint-Ouen (Somme) : cinq occupations paléolithiques du début de la dernière glaciation. Paris, éd. de la Maison des sciences de l'Homme (Documents d'archéologie française, 90), p. 16-39.

Avtoine P., Munalt A-V., SommÉ. J. 1994 : «Réponse des environnements aux climats du Début Glaciaire weichsélien: données de la France du Nord-Ouest ». Quaternaire, 5, 3-4, p. 151-156.

ANTOHN. P., Locht J.-L., SWIN.Nen C.

1995a: "Le gisement paléolithique moyen de Bettencourt-Saint-Ouen (Somme, France). Chronostratigraphie et caractérisation des industries lithiques ". Notae Praehistoricae, 15, p. 141-153.

Antolne P., Munaut A.-V., Van KOL.FSCHOTEN T., LimoNdiN N.

1995b: "Une occupation du Paléolithique moyen en contexte fluviatile dans la séquence de la très basse terrasse de la Somme à Saint-Sauveur (Somme) ". Bulletin de la Société préhistorique française, 92, 2, p. 201-212.

Antoine P., Lautridou J.-P., Sommé J., Auguste P., Auffret J.-P., Baize S., Ci.et-Pellerin M., Coutard J.-P., Dewol. Y Y., Dugue O., Joly F., Laignel. B., LAURENT M., LaVOll.́. M., Lebret P., LÉcolle F., Lefebv'Re D., Limondin-Lozolet N., Munalt A.-V., Ozolf J.-C., Quesnel. F., Rousseac D.-D.

1998 : «Le Quaternaire de la France du Nord-Ouest : limites et corrélations". Quaternaire, 9, p. 227-241.

Antoine P., Rousseau D.-D., Lautridol J.-P., Hatté C.

1999 : «The Last Interglacial Glacial Climatic Cycle in Loess-Palaeosols Successions of Northwestern France ». Boreas, 4, p. 551-563.

ANTOINe P., Rolsseau D.-D., ZÔLler L., Lang; A., Munaut A.-V., Hatté C., FONTLGNE M.

2001 : " High Resolution Record of the Last Interglacial-Glacial Cycle in the Nussloch Loess Palaeosol Sequences, Upper Rhine Area Germany ". Quaternary International, 76-77, p. 211-229.
Bahain J.J., Dwrila G., Ramond P.

1996 : Les gisements pléistocènes de VilliersAdam (Val-d'Oise). Rapport de diagnostic archéologique. AFAN, Service départemental de l'Archéologie du Vald'Oise, Service régional de l'Archéologie d'île-de-France, $40 \mathrm{p}$.

\section{BALEscu S.}

1988 : Apports de la thermoluminescence à la stratigraphie et à la sédimentologie des loess du Nord-Ouest de l'Europe. Thèse de doctorat de sciences, univ. de Bruxelles, vol. I : 199 p.; vol. II : annexes, $144 \mathrm{p}$.

\section{BARBALIT R.}

1995 : Écologie des peuplements. Structure et dynamique de la biodiversité. Paris, Masson, 273 p.

BARTHÉLEMY L.

1985 : «Réflexions sur la répartition du pollen. Conséquences pour l'archéologie ", in Palynologie archéologique. Paris, éd. du CNRS, p. 53-86.

BEAUlieu J.-L. DE

1977 : Contribution pollenanalytique $\grave{a}$ l'histoire tardiglaciaire et holocène de la végétation des Alpes méridionales fransaises. Thèse, univ. d'Aix-Marseille III, 358 p. et annexes.

Beallifeu J.-L. DE, Pons A., Reille M.

1982 : «Recherches pollenanalytiques sur l'histoire de la végétation de la bordure nord du Massif du Cantal (Massif central, France) ". Pollen et Spores, 24, p. 251-300.

1985 : « Recherches pollenanalytiques sur l'histoire tardiglaciaire et holocène de la végétation des monts de l'Aubrac (Massif central, France) ". Revue de Paléobotanique et de Palynologie, 44, p. 37-80.

Becze-Dfak J., LANGohr R., VERRECHIA E. P.

1997 : “Small Scale Secondary $\mathrm{CaCO}_{3}$ 
Accumulations in Selected Sections of the European Loess Belt. Morphological Forms, and Potential for Paleoenvironmental Reconstruction ». Geoderma, 76, p. 221-252.

\section{BthrF K. E.}

1989 : "Biostratigraphy of the Last Glacial Period in Europe ". Quaternary Sciences Reviews, 8, p. 25-44.

Birks H. J. B., Birks H. H.

1980 : Quaternary Palaeoecology: Londres, éd. E. Arnold, 289 p.

BOËDA É.

1993 : "Le débitage discoïde et le débitage Levallois récurrent centripète ". Bulletin de la Société préhistorique francaise, 90, 6, p. 392-404.

1994 : Le concept Levallois : variabilité des méthodes. Paris, CNRS Éditions (Monographie du CRA, 9), 280 p.

1997 : Technogenèse des systemes de production lithique au Paléolithique inférieur et moyen en Europe occidentale et au Proche-Orient. Mémoire d'habilitation à diriger des recherches, univ. Paris X-Nanterre, 173 p., 87 fig.

Bonis A., Dechamane: S., Godert S. 1992 : Trente ans d'archéologie en Val-d'Oise (1960-1989). Saint-Ouen-l'Aumône, Service départemental d'Archéologic du Val-d'Oise, 1, 99 p.

\section{BOTTEMA S.}

1975 : «The Interpretation of Pollen Spectra from Prehistoric Settlements (with Special Attention to Liguliflorac) ". Palaeohistoria, 17, p. 18-35.

\section{BoYCOTT A. E.}

1934 : "The Habitats of Land Mollusca in Britain ». The Journal of Ecology, 22, p. $1-38$

\section{CANTI M.}

1998 : "Origin of Calcium Carbonate Granules Found in Buried Soils and Quaternary Deposits ". Boreas, 27, p. 275-288.
Caspers G., Frelind H.

2001 : "Vegetation and Climate in the Early- and Pleni-Weichselian in Northern Central Europe ". Boreas, 16, p. 31-48.

Cavilatr C.

1967 : Carte géologique de la France au 1/50 000, feuille de Creil, XVIII-12 (127). Orléans, notice du BRGM, $23 \mathrm{p}$.

Cavelifer C., Labolrgugane J., Mégnien C., Mégnien F., Pomiroi. C., WYNS R.

1980 : “Éocène supérieur " in Mrignien C. (dir.), Synthèse géologique du Bassin de Paris. Mémoires du BRGM, 101, 1, p. 379-399.

Cicchisi M.

1990 : "Le Paléolithique en Pays de France ". Bulletin de la Jeunesse préhistorique et géologique de France, 2, $66 \mathrm{p}$.

\section{ChaRplintile V.}

1982 : Étude du Paléolithique inférieur et moyen dans la basse vallée de l'Oise. Mâtrise d'archéologie préhistorique, univ. Paris I.

\section{Chathaunfe J-J.}

1974 : Éléments de palynologie. Applications géologiques. Laboratoire de paléontologie de l'université de Genève, cours de $3^{r}$ cycle en sciences de la terre, $345 \mathrm{p}$.

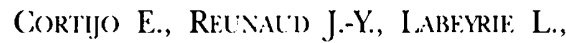
Pahliakd D., LehmaN B., Cremer M., Grousser F.

1995: "Étude de la variabilité climatique à haute résolution dans les sédiments de l'Atlantique Nord ". Comptes rendus de l'Académie des Sciences de Paris, Série IIa, 321, p. 231-238.

\section{COL'R P.}

1974 : "Nouvelles techniques de détection des flux et des retombées polliniques: étude de la sédimentation des pollens et des spores à la surface du sol ". Pollen et Spores, 16, p. 103-141.
Couteacx M.

1977 : «À propos de l'interprétation des analyses polliniques de sédiments minéraux, principalement archéologiques ", in Approche écologique de l'Homme fossile. Paris, suppl. au Bulletin de l'Association française pour l'étude du Quaternaire, p. 259-276.

DAGE:I J.

1979 : Les modèles mathématiques en écologie. Paris, Masson (coll. Écologie), 2“éd., $172 \mathrm{p}$.

DANSGAMRD W., JOHNSEN S. J., Clausen H. B., Dahl-Jensen D., Gundestrup N. S., Hammerammik C. U., Hvidbjerg; C. S., StrFFenstiN J.-P., SveinbjøRnSDOtTIR A. E., JOL\%.I.J., BOND G.

1993 : «Evidence for General Instability of Past Climate from a 250-kyr IceCore Record ". Nature, 364, p. $218-220$.

DFloze V., Depakpe P., (Golledo J.-M., KRIER V., LOCHT J.-L. (DIR.)

1994 : Le Paléolithique moyen dans le nord du Sénonais : contexte géomorphologique, industries lithiques et chronostratigraphie. Paris, éd. de la Maison des sciences de l'Homme (Documents d'archéologie française, 47), $267 \mathrm{p}$.

Depakip: P., DFschodT L.

2001 : « Le gisement paléolithique moyen de Gouy-Saint-André "Le Brétigny" (Pas-de-Calais) ", in Paléolithique et Mésolithique du nord de la France : nouvelles recherches. Lille, univ. des sciences et technologies (Publications du Centre d'études et de recherches préhistoriques, 6), p. 185-198.

Dibbi.e H., BaR-Yosef O.

1995 : The Definition and Interpretation of Levallois Technology: Madison (Wisconsin), Prehistory Press (Monographs in World Archaeology, 23), $502 \mathrm{p}$.

DRIESSEN P. M., DUDAL. R.

1991 : "Greyzems ", in Dritssen P. M., DudAl, R. (eds), The Major Soils of the 
World, Agricultural University Wageningen, dep. of Soil Science and (jeology, p. 245-248.

Dlrbet G., Rodriglez P., Badaliax L., Hadjolis D., Galthile A., Lalirh.NT M., Ricard J.-L., WatTE. J. 1997 : « Découverte d'un site Paléolithique moyen dans des alluvions saaliennes du confluent Seine-Marne à Maisons-Alfort (Valde-Marne) ". Comptes rendus de l'Académie des Sciences de Paris, 324, série IIa, p. 505-512.

E.HAII H.

1968 : Biogéographie, Paris, éd. A. Colin (coll. U), $406 \mathrm{p}$.

Engelmann A., Frichen M.

1998 : "Datations TL/IRSL ", in ANTOINE P. (éd.), Le Quaternaire de la vallée de la Somme et du littoral picard. Livret-guide de l'excursion de l'Association française pour l'étude du Quaternaire dans le bassin de la Somme, Amiens, 1998, p. 41-42.

Engeimanis A., Frechen M., Antoine P. 2000 : "Chronostratigraphie früweichselzeitlicher kolluvialer Sedimente von Bettencourt-Saint-Ouen (NordFrankreich) ", in BECKE:R-HaUman R., FRECHEX M. (éd.), Terrestrische Quartärgeologie, Köln, éd. Logabook, p. 12-22.

FAEGRI K., IVIRSFR J.

1989 : Textbook of Pollen Analysis. IV"ed. by Faegri K., Emilkaland P. and Krzywinski K., Chichester, New-York, Brisbanne, Toronto, Singapore, Ltd J. Wiley and Sons, 328 p.

\section{FAO-UNESCO}

1997 : Colour Chart of the 106 Soil Unit of the FAO-UNESCO Soil Map of the World Legend. Amsterdam, Elsevier Science Publishers.

Fittrir R., Fitter A., Farrer A.

1991 : Guide des Graminées, Carex, Joncs, Fougères. Paris, éd. Delachaux et Niestlé, $256 \mathrm{p}$.
Fol.teri M., MAGiRI D., SADORI L.

1988 : « 250,000-year pollen record from valle di Castiglione (Roma) ». Pollen et Spores, 30, p. 329-356.

\section{ForrstitR H.,}

1993 : " Le Clactonien : mise en application d'une nouvelle méthode de débitage s'inscrivant dans la variabilité des systèmes de production lithique du Paléolithique ancien ". Paléo, 5, p. 53-82.

FolRnile P.

1977 : Les Quatre flores de France. Paris, éd. Lechevalier, vol. I: texte, 105 p.; vol. II : atlas, $308 \mathrm{p}$.

FRECHEN M.

1999 : "Upper Pleistocene Loess Stratigraphy in Southern Germany ". Quaternary Geochronology, 18, p. 243-269.

Gerasimova M. I., Gubin S. V., ShIOba S. A.

1996 : "Soils of Northern Forest-Steppe Grey forest-soils ", in MikdemA R. (éd.), Soils of Russia and Adjacent Countries: Geography and Micromorphology; Moscou-Wageningen, 1, p. 23-134.

Gouf́do J.-M., Bats J.-C., Krier V., PERNot P., Ricard J.-L.

1994 : "Le gisement moustérien de la "Butte d'Arvigny", commune de Moissy-Cramayel (Seine-et-Marne) : premiers résultats ". Bulletin de la Société préhistorique française, 91, p. $369-377$.

GroOTES P. M., SIlliter M., White J. V. C., JOHNSEN S., JOUZi. J.

1993 : " Comparison of Oxygene Isotope Records from the GISP2 and GRIP Greenland Ice Cores ». Nature, 336, p. 552-554.

Grlas-Cavagingto C.

1968 : “Étude palynologique des divers gisements du Sparnacien du Bassin parisien ". Mémoire de la Société géologique de France, $n^{\circ}$ spécial 47, 4, mém. $n^{\circ} 110,144 \mathrm{p}$.
1977 : «Étude palynologique de l'Éocène du Bassin anglo-parisien ". Mémoire de la Société géologique de France, $\mathrm{n}^{\circ}$ spécial 56 , mém. $\mathrm{n}^{\circ} 131$, $64 \mathrm{p}$.

\section{Gullentops F.}

1954 : Contribution à la chronologie du Pléistocène et des formes de relief en Belgique. Université de Louvain (Mémoire de l'Institut de géologie, $18)$, p. $125-252$.

\section{HAESAERTS P.}

1985 : "Les lœss du Pléistocène Supérieur en Belgique ; comparaison avec les séquences de l'Europe Centrale ". Bulletin de l'Association française pour l'étude du Quaternaire, 22, p. 105-115.

HaFsakets P., Mestdagh H.

2000 : "Pedosedimentary Evolution of the Last Interglacial and Early Glacial Sequence in the European Loess Belt from Belgium to Central Russia ". Geologie and Mijnbouw/Netherlands Journal of Geosciences, 79, p. 313-324.

Hafsaerts P., Mestdach II., Bosqleit D. 1999 : «The Sequence of Remicourt (Hesbaye, Belgium) : New Insights on the Pedo- and Chronostratigraphy of the Rocourt Soil ". Geologica Belgica, 2-3, p. 5-27.

Haest R., Munalt A.-V., Humbans I., Gullentops F., MOOK W. G.

1986 : « La Stratigraphie de Beerse-Dam (Belgique) ". Bulletin de l'Association fransaise pour l'étude du Quaternaire, 25-26, p. 158-167.

HALl S. A.

1981 : "Deteriorated Pollen Grains and the Interpretation of Quaternary Pollen Diagrams ». Review of Palaeobotany and Palynology, 32, p. 193-206.

HATTÉ C.

2000 : Les isotopes du carbone $\left({ }^{14} \mathrm{C}\right.$ et ${ }^{13} \mathrm{C}$ ) dans la matière organique des loess de l'Europe du Nord-Ouest; applications paléoclimatiques. Thèse de doctorat en sciences, univ. Paris XI. 
Hatté C., Fontugne M., Rousseau D.-D., Antoine P., Zöller L., TisnératLABORDE N., BENTALEB I.

1998 : $~{ }^{13} \mathrm{C}$ Variations of Loess Organic Matter as a Record of the Vegetation Response to Climatic Changes during the Weichselian ». Geology, 26, p. $583-586$.

Hatté C., Antoine P., Fontugne M.-R., Rousseau D.-D., TISNÉratLABORDE N., ZÖlLER L.

1999 : «New Chronology and Organic Matter ${ }^{13} \mathrm{C}$ Paleoclimatic Significance of Nußloch Loess Sequence (Rhine Valley, Germany) ". Quaternary International, 62, p. 85-91.

Hatté C., Antoine P., Fontugne M.-R., LANG A., ROUSSEaU D.-D., ZÖll.ter L. 2001 : $~{ }^{13} \mathrm{C}$ Variation of Loess Organic Matter as a Potential Proxy for Paleoprecipitation ". Quaternary Research, 55, p. 33-38.

HEIM J.

1970 : Les Relations entre les spectres polliniques récents et la végétation actuelle en Europe occidentale. Liège, éd. Derouaux, $200 \mathrm{p}$.

HuETZ DE LEMPS A.

1994 : Les Paysages végétaux du globe. Paris, Masson, $2^{\mathfrak{c}}$ éd., $182 \mathrm{p}$.

Huijzer A. S.

1993 : Cryogenic Microfabrics and Macrostructures : Interrelations,

Processes, and Paleoenvironmental Significance. $\mathrm{PhD}$ Thesis Vrije Universiteit Amsterdam, 245 p.

Huntley B., BiRks H. J. B.

1983 : An Atlas of Past and Present Pollenmaps for Europe: 0-13 $000 \mathrm{BP}$ Years ago. Cambridge, University Press, vol. I : 667 p. ; vol. II : annexes.

JOI.L.Y M.-C.

1994 : Variations holocènes de la limite supérieure de la forêt sur les hauts versants $d u$ Cantal d'après l'analyse pollinique. Paris, Travaux du laboratoire de géographie physique de l'université Paris 7, 138 p.
KERNEY M. P.

1971 : «A Middle Weichselian Deposit at Halling, Kent ". Proceedings of the Geologists'Association, 82, p. 1-11.

Kerney M. P., Cameron R. A. D., JUNGBLUTH J. H.

1983 : Die Landschnecken Nord-und Mitteleuropas. Hamburg - Berlin, éd. Paul Parey, 384 p.

Kolstrup E.

1980 : " Climate and Stratigraphy in Nothwestern Europe between 30000 B.P. and 13000 B.P., with Special Reference to the Netherlands ". Mededlingen von de Rijks Geologische Dienst, 32, p. 181-253.

Kolstrup E., WijMSTRA T. A.

1977 : “ A Palynological Investigation of the Moershoofd, Hengelo and Denekamp Interstadials in Netherlands ". Geologische Mijnbouw, 56, p. 85-102.

\section{LAUTRIDOU J.-P.}

1985 : Le cycle périglaciaire pléistocène en Europe et plus particulièrement en Normandie. Thèse de doctorat d'État, univ. de Caen, Centre de géomorphologie, 2 vol., 908 p.

LAVILLE A.

1898 : «Le gisement chelléo-moustérien à Corbicules de Cergy (Seine-etOise) ". Bulletin de la Société d'anthropologie de Paris, $4^{\mathrm{e}}$ série, 9 , p. 56-68.

1902 : “Réponse à Monsieur Rutot sur son étude géologique et anthropologique du gisement de Cergy (Seine-et-Oise) ". Bulletins et Mémoires de la Société d'anthropologie de Paris, $5^{\mathrm{e}}$ série, 3, p. 742-749.

Lebret P., Hal.bout $\mathrm{H}$

1991 : "Le Quaternaire dans le Vald'Oise ". Bulletin du Centre de géomorphologie de l'université de Caen, 39-40, $267 \mathrm{p}$

LEROI-GOURHAN ARL.

1988 : «L'apport des pollens dans le gisement paléolithique moyen de
Seclin (Nord) ». Revue archéologique de Picardie, 1-2, p. 57-61.

LiMONDIN N.

1995 : " Late-Glacial and Holocene Malacofaunas from Archaeological Sites in the Somme Valley (North France) ". Journal of Archaeological Science, 22, p. 683-698.

LIMONDIN-LOZOUET N.

1997 : " Mollusca in Archaeological Context: Tools for Palaeoenvironmental Reconstructions. The Example of "Le Closeau", a Prehistoric Site in the Seine Valley (France) ». Heldia, 4, p. 166-171.

LIMONDIN N., ROUSSEAU D.-D.

1991 : « New Holocene Malacological Sequence at Verrières, Seine Valley, France ", Boreas, 20, p. 207-229.

LiN B., Liv R., AN Z.

1991 : "Preliminary research on stable isotopic compositions of Chinese loess ", in Liu T. (éd.), Loess, Environment and Global Change, Pékin, Science Press, p. 124131.

LOCHT J.-L. (DIR.)

2002 : Bettencourt-Saint-Ouen (Somme, France) : cinq occupations paléolithiques du début de la dernière glaciation. Paris, éd. de la Maison des sciences de l'Homme (Documents d'archéologie française, 90), 172 p., 145 fig.

LOCHT J.-L., ANTOINE P.

2001 : « Caractérisation technotypologique et position chronostratigraphique de plusieurs industries à rares bifaces et amincissements bifaciaux du nord de la France ", in Cliquet D. (dir.), Les industries $a$ outils bifaciaux du Paléolithique moyen d'Europe occidentale. Actes de la tableronde organisée à Caen, 14-15 oct. 1999. Liège (Études et recherches archéologiques de l'université de Liège, 98), p. 129-134.

Locht J.-L., ANTOINE P., SWINNen C. 1995a : "Le gisement paléolithique de Plachy-Buyon (Somme) ". Revue archéologique de Picardie, 3-4, p. 3-33. 
Locht J-L., SWincen C., ANtoine P., Acciste P., Patol-Mathis M., DepatPE P., Fal.gètres C., LALRF.NT M., BAHAIN J.-J.

1995b: «Le gisement Paléolithique moyen de Beauvais (Oise) ". Bulletin de la Société préhistorique française, 92 , 2, p. 213-226.

Locht J.-L., Bahain J.-J., Drwila G., Raymond P., Antoine P., Caspar J.-P., Debrinham N., Galthier A., Krier V., LiMONDIN N.

1997 : Le gisement paléolithique moyen du Petit Saule et la séquence pléistocène du Chamesson de Villiers-Adam (Val-d'Oise). Document final de synthèse, AFAN, Saint-Denis, Service régional de l'Archéologie d'île-de-France, SDAVO, $76 \mathrm{p}$.

Locht J.-L., Swinnen C., Antoine P., RÉvil.lion S., Depaepe. P.

2001 : « Le gisement paléolithique moyen de Bettencourt-Saint-Ouen (Somme) ". in Paléolithique et Mésolithique du nord de la France: nouvelles recherches. Lille, univ. des sciences et technologies (Publications du Centre d'études ct de recherches préhistoriques, 6), p. 199-237.

LOWE J.J., WAL.KER M. J. C.

1997 : Reconstructing quaternary environments. Harlow, Addison Weslmey Limited, éd. Longmann Limited, $2^{\circ}$ éd., $446 \mathrm{p}$.

LOZFK V.

1964 : Quartärmollusken des Tschechoslowakei. Prague, Akademie der Wissenschaften, 374 p.

1972 : Le lœss et les formations assimilées: corrélations entre l'Europe centrale et la France par la faune de mollusques, in Études sur le Quaternaire dans le monde. Proceedings of the VIII I Inqua Congress, Paris 1969, p. 579-606.

1990 : " Molluscs in Loess, their Paleoecological Significance and Role in Geochronology. Principles and Method ". Quaternary International, 7-8, p. 71-79.
2001 : "Molluscan Fauna from the Loess Series of Bohemia and Moravia ». Quaternary International, 76-77, p. 141-156.

Marcy J.-L., Auglste P., Fontlgne M., MLNALT A.-V., VAN Vl.IET-LANoË. B.

1993 : " Le Gisement moustérien d'Hénin-sur-Cojeul (Pas-de-Calais)". Bulletin de la Société préhistorique francaise, 90, 4, p. 251-256.

Martinson D. G., Pisias N. G., Hays J. D., Imbrif J., MOORE T. C., SHACKLETON N. J.

1987 : "Age Dating and the Orbital Theory of the Ice Ages: Development of a High-Resolution 0 to 300,000-year Chronostratigraphy ". Quaternary Research, 27, p. 1-29.

Mercier N., Valladas H., Valladidas G. 1995 : «Flint Thermoluminescence Dates from the CFR Laboratory at Gif: Contributions to the Study of the Chronology of the Middle Palaeolithic ". Quaternary Science Reviews, 14, p. 351-364.

Michel. J.-P.

1972 : Le Quaternaire de la région parisienne. Thèse de doctorat d'État en géologie, univ. Paris 6, $572 \mathrm{p}$.

Moorf. P. D., Webb J. A., Collison M. E. 1991 : Pollen Analysis. Blackwell Scientific Publications, $2^{\circ}$ éd., 216 p.

\section{MORZADECG-KFRFOURN M.-T.}

1977 : «Remarques concernant la corrosion des grains de pollen dans les sédiments soumis à l'altération " in Approche écologique de l'Homme fossile. Paris, suppl. au Bulletin de l'Association française pour l'étude $d u$ Quaternaire, p. 51-52.

MLNAUT A.-V.

1974 : «Les analyses palynologiques du sol gris de Saint-Sauveur ". Bulletin de l'Association fransaise pour l'étude du Quaternaire, 40-41, p. 232-235.

1996 : “Palynologie » in Deschont L. (éd.), Le Quaternaire de Lambersart "Les Conquérants". Cahiers de Préhistoire du Nord, 19, p. 3437.
Mirton J. B., Frinch H. M.

1992 : "Thaw Modification of FrostFissure W'edges, Richard Island, Pleistocene Mackenzie Delta, Western Arctic Canada ". Journal of Quaternary Science, 8, p. 185-196.

1993 : « Thermokarst Involutions : Summer Island, Pleistocene Mackenzie Delta, Western Canadian Arctic ». Permafrost and Periglacial Processes, 4, p. $217-229$.

OL.DFIEL.D F.

1964 : "Late-Quaternary Deposits at Le Moura, Biarritz, South-West France ". The New Phytologist, 63, p. 374-409.

O\%F.N1) P.

1964 : Biogéographie végétale. Paris, éd. Doin (coll. Biologie), 369 p.

1994 : Végétation du continent européen. Paris, éd. Delachaux et Niestlé, 271 p.

Plaisance G., Canlleux A.

1958 : Dictionnaire des sols. Paris, éd. La Maison rustique, $604 \mathrm{p}$.

PoKROVSKAIA I. M.

1958 : Analyse pollinique. Paris, Annales du Service d'information géologique du BRGM, 24, 435 p.

Pomerol. C., Felcuevr L.

1974 : Guides géologiques régionaux - Bassin de Paris, Ille-de-France, Pays de Bray. Paris, Masson, 222 p.

Pons A.

1984 : « À Propos de l'apport de la palynologie quaternaire à la connaissance de la forêt bourguignonne ". Bulletin de la Société française de botanique, 131, Lettres botaniques, 1, p. $49-53$.

Prefecr. R. C.

1990 : "The Molluscan Fauna of Late Devensian Loess from Reculver, Kent ». Journal of Conchology, 33, p. $295-297$.

PLissÉcitr J.-J.

1976: Mollusques continentaux quaternaires de Bourgogne. Paris, Doin (Mémoires de géolologie de l'université de Dijon, 3), 241 p. 
1978 : " Les mollusques des séries lœssiques à Achenheim ". Recherches géographiques à Strasbourg, 7, p. 55-76.

\section{REILILE M.}

1978 : « À propos de la disparition du pollen dans certains sédiments minéraux ". Annales des mines de Belgique, 6, p. 707-712.

1990a: Lesons de palynologie et d'analyse pollinique. Paris, éd. du CNRS, 206 p.

1990b: " La Tourbière de La Borde (Pyrénées orientales, France) : un site clé pour l'étude du Tardiglaciaire sud-européen ". Comptes rendus de l'Académie des sciences de Paris, 310, Série II, p. 823-829.

1992 : Pollen et spores d'Europe et d'Afrique du Nord. Marseille, Laboratoire de botanique historique et palynologie, $520 \mathrm{p}$.

1995 : Pollen et spores d'Europe et d'Afrique du Nord, supplément 1. Marseille, Laboratoire de botanique historique et palynologie, $327 \mathrm{p}$.

1998 : Pollen et spores d'Europe et d'Afrique du Nord, supplément 2. Marseille, Laboratoire de botanique historique et palynologie, $521 \mathrm{p}$.

ReIll.e M., BrathIFU J.-L. DE, PONS A.

1985 : «Recherches pollenanalytiques sur l'histoire tardiglaciaire et holocène de la végétation du Cézallier, de la Planèze de Saint-Flour et de la Margeride (Massif central, France) ". Pollen et Spores, 27, p. 209-270.

RÉVILIIION S

1994 : Les industries laminaires au Paléolithique moyen en Europe septentrionale. L'exemple des gisements de Saint-Germain-des-Vaux / Port Racine (Manche), de Seclin (Nord) et de Riencourt-lès-Bapaume (Pas-de-Calais). Lille, univ. des sciences et technologies (Publications du Centre d'études et de recherches préhistoriques, 5), $187 \mathrm{p}$.

Rousseau D.-D.

1987 : " Paleoclimatology of the Achencheim Series (Middle and Upper Pleistocene, Alsace, France).
A Malacological Analysis ". Palaeogeography, Palaeoclimatology, Palaeoecology, 59, p. 293-314.

1989a: "Réponses des malacofaunes terrestres quaternaires aux contraintes climatiques en Europe septentrionale ". Palaeogeography, Palaeoclimatology, Palaeoecology, 69, p. 113-124.

1989b: "Estimation quantitative des paléotempératures hivernales en Alsace depuis 340000 ans à partir de l'analyse des associations malacologiques ". Comptes rendus de l'Académie des sciences de Paris, 309, série II, p. 1623-1628.

1990 : « Statistical analyses of loess molluscs for paleoecological reconstructions ". Quaternary International, 7-8, p. 81-89.

Roussead D.-D., KeEN D. H.

1989 : "Malacological Records from the Upper Pleistocene at Portelet (Jersey, Channel Islands) : Comparisons with Western and Central Europe". Boreas, 18, p. 61-66.

Rousseat: D.-D., Puisségur J.-J. 1990 : «A 350,000-year Climatic Record from the Loess Sequence of Achenheim, Alsace, France ". Boreas, 19 , p. 203-216.

Rousseau D.-D., Puisséc;ur J.-J., LALTRIDOU J.P.

1990 : « Biogeography of the Pleistocene Pleniglacial Malacofaunas in Europe. Stratigraphic and Climatic Implications ". Palaeogeography, Palaeoclimatology, Palaeoecology, 80, p. 7-23.

Rousseal D.-D., KlKi.A G., ZÖll.er L., Hradilova J.

1998a : "Early Weichselian Dust Storm Layer at Achenheim in Alsace, France ". Boreas, 27, p. 200-207.

Rousseau D.-D., Zöll.er L., VAl.et J.-P. 1998b: " Late Pleistocene Climatic Variations at Achenheim, France, Based on a Magnetic Susceptibility and TL Dating ". Quaternary Research, 49 , p. 255-263.
RUFFALDI P.

1994 : “ Relationship between Recent Pollen Spectra and Current Vegetation around the Cerin Peat Bog (Ain, France) ". Review of Palaeobotany and Palynology, 82, p. $97-112$.

Rutot M. A.

1902 : «Étude géologique et anthropologique du gisement de Cergy (Seine-et-Oise) ". Bulletin de la Société d'anthropologie de Bruxelles, 20, p. 1-57.

SCHIRMER W.

2000 : « Rhein Loess, Ice Cores and Deep-Sea Cores during MIS 2-5». Zeitschrift der deutschen geologischen Gesellschaft, 151, p. 309-332.

ShUGalei L. S.

1997 : «Ecological Features of Gray Forest Soils in the Forest-Steppe Zone of Central Siberia ". Eurasian Soil Science, 31, p. 209-218.

SOMmÉ J., PAipe: R., LAutridou J.-P.

1980 : «Principes, méthodes et système de la stratigraphie du Quaternaire dans le Nord-Ouest de la France et la Belgique ", in Problemes de stratigraphie quaternaire en France et dans les pays limitrophes. Paris, suppl. 1 au Bulletin de l'Association française pour l'étude du Quaternaire, p. 148-162.

Sommé J., Munaut A.-V., Emontspohl. A.-F., Limondin N., Lefevre D., Cunat-Bogk N., MOUThon J., GILOT E.

1994: " - The Watten Boring - an Early Weichselian and Holocene climatic and palaeological record from the French North Sea coastal plain ". Boreas, 23, p. 231-243.

SOYER R.

1959 : Notice explicative de la carte géologique i $1 / 50000$ de L'Isle-Adam (XXIII-13), BRGM, $12 \mathrm{p}$.

Suc.J.-P.

" Origin and Evolution of the Mediterranean Vagatation and 
Climate in Europe ". Nalure, 307 $(5950)$, p. $429-432$.

TEDROW J. C. F.

1977 : Soils of the polar landscapes. New Brunswick, New Jersey, Rutgers University Press, $638 \mathrm{p}$.

TIFFREAl A. (DIR.)

1993 : Riencourt-les-Bapaume (Pas-de(alais). Un gisement du Paléolithique moyen. Paris, éd. de la Maison des sciences de l'Homme, (Documents d'archéologie française, 37), $128 \mathrm{p}$.

Tifrekal A., Révillon S., Sommé J., Van VIIT:I-I ANOË: B.

1994 : "Le gisement paléolithique moyen de Seclin (Nord) ". Bulletin de la Société préhistorique française, 91, 1, p. 23-46.

VAN CAMPO M.

1984: "Relations entre la végétation de l'Europe et les températures de surface océaniques après le dernier maximum glaciaire ". Pollen et Spores, 26, p. $497-518$.
VAN dr:k Hamare T.

1995: "The Dinkel Valler Revisited: Pleniglacial Stratigraphy of the Eastern Netherlands and Gilobal Climatic Change ". Mededlingen von de Rijks (jeologische Dienst, 52, p. 343-355.

VAN dek HaMmex T., WijMSTRA T. A., ZACiNIJN W: $\mathrm{H}$.

1971 : "The Floral Record of the Late Cenozoíc of Europe ", in Tirkkmax K. K. (éd.), The Late Cenozoüc glacial ages. New Haven London, Yale University Press, p. 391-424.

\section{VAN PEik P.}

1992 : The Levallois Reduction Strategy. Madison, Wisconsin, Prehistory Press (Monographs in World Archaeology, 13), $137 \mathrm{p}$.

VAN VIIIFI-I ANOË B.

1987 : Le rôle de la glace de ségrégation dans les formations superficielles de l'Europe du Nord-Ouest. Thèse de doctorat d'État, univ. Paris 1, $864 \mathrm{p}$.
1990 : «Le pédocomplexe de Warneton : où en est-on : Bilan paléopédologique et micromorphologique ". Quaternaire, 1, p. 65-76.

1996 : «Dynamics and Extent of the Weichselian Permafrost in Western Europe (Substage 5e to Stage 1)". Quaternary international, 3-4, p. $109-113$.

Whinc; H., Ambrose S. H., I.I C. I.. J., FOLLLIER I. R.

1997 : «Paleosol Stable Isotope Evidence for Early Hominid Occupation of East Asian Temperate Environments ". Quaternary Research, 48, p. $228-238$.

WiNs R., MoNCiARDINi G.

1979 : Carte géologique de la France, Feuille de Méru XXI-12 (126). Orléans, notice du BRGM, 61 p.

W'OLLARI) G., MOOK W'

1982 : "Carbon Dates at Grande Pile: Correlation of Land and Sea Chronologies ". Science, 215, p. 159-161. 